\title{
EAST93 - GEOPHYSICAL TRAVERSE FROM THE TRANSANTARCTIC MOUNTAINS TO THE WILKES BASIN, EAST ANTARCTICA
}

\section{A Joint United States - New Zealand Science Project}

\section{Uri ten Brink}

U.S. Geological Survey

Woods Hole, MA 02543, USA

\section{Stephen Bannister}

Institute of Geological and Nuclear Sciences P.O. Box 1320, Wellington, New Zealand

With appendices prepared by:

Mike Collins, Rebecca Drury, Ron Hackney

Bill King, Yizhaq Makovsky, and John West

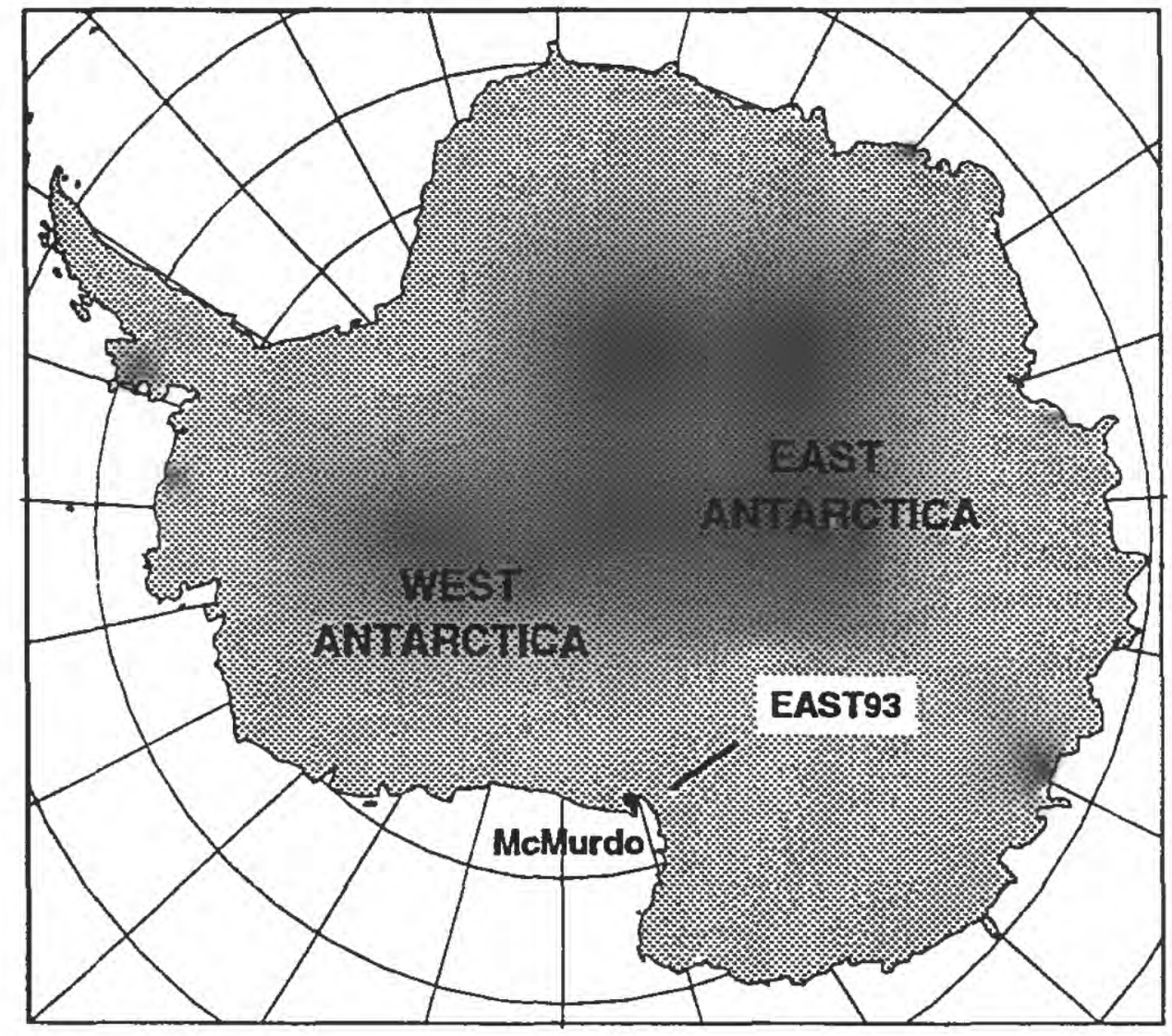

U.S. GEOLOGICAL SURVEY

OPEN-FILE REPORT 95-225

April 12, 1995

This report is preliminary and has not been reviewed for conformity with U.S. Geological Survey editorial standards (or with the North American Stratigraphic Code). Any use of trade, product, or firm names is for descriptive purposes only and does not imply endorsement of the U.S. Government. 


\section{TABLE OF CONTENTS}

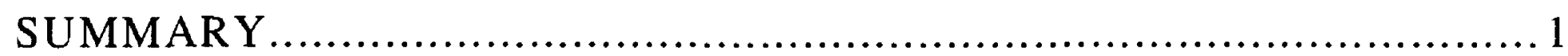

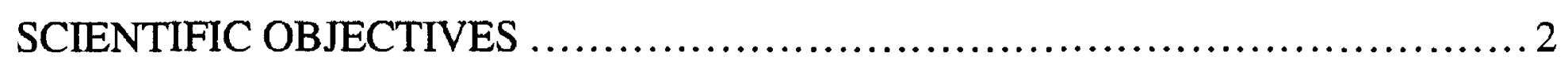

PREVIOUS WORK IN THE AREA .......................................... 2

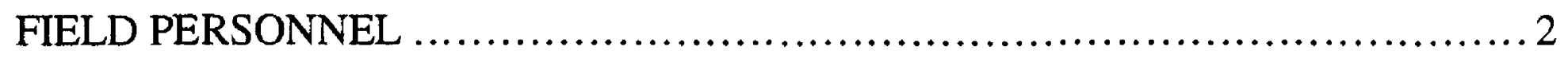

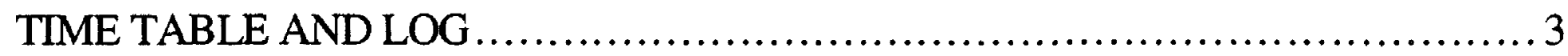

INSTRUMENTATION

Location and Surveying ......................................... 4

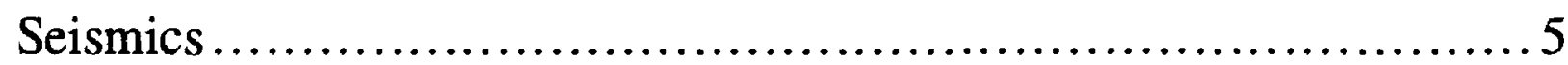

Gravity........................................................ 6

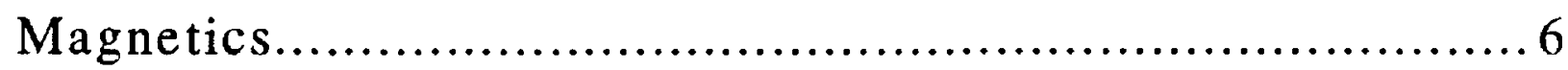

Ground penetrating radar ....................................... 6

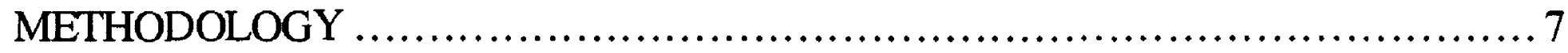

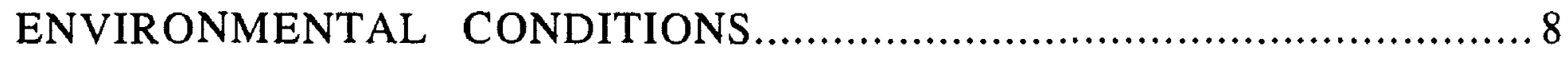

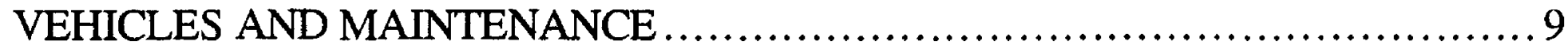

LESSONS AND RECOMMENDATIONS

Seismic Work ................................................... 9

Effect On Humans.................................................. 10

Logistics..................................................... 11

Mechanical Aspects ........................................... 11

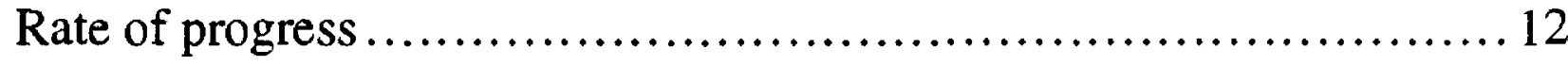

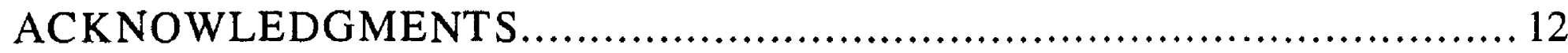

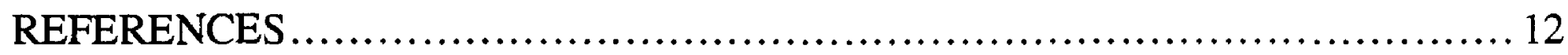

APPENDIX 1 Locations of surveyed positions, shot numbers, and magnetic readings, By John West and Rebecca Drury.

APPENDIX 2 Gravity measurements, by Ron Hackney and Uri ten Brink.

APPENDIX 3 Ground penetrating radar measurements, by Yizhaq Makovsky

APPENDIX 4 Ground conditions, by Ron Hackney.

APPENDIX 5 Weather observations by the main party, by Ron Hackney and Rebecca Drury

APPENDIX 6 Weather observations by the advance party, by Bill King.

APPENDIX 7 Barometric pressure and temperatures at the temporary base camp averaged over 6 hours, by Rebecca Drury and Ron Hackney.

APPENDIX 8 Mechanic's report, By Mike Collins 


\section{LIST OF FIGURES}

Figure 1. After page 1. Isostatically-adjusted bedrock elevation map of East Antarctica next to the Transantarctic Mountains (from Drewry,1983). The location of the traverse is shown by the heavy line, and the location of the $1958 / 59$ traverse is shown by the light line.

Figure 2. After page 2. Surface elevation map of East Antarctica in the vicinity of the traverse (100 m Contour interval). Map was contoured from digitized values of elevation collected by the NSF/SPRI/TUD airborne radio echo-sounding surveys in 1971-72 and 1975 (D. Vaughan, SPRI, written comm., 1993). Heavy line - location of traverse. Dotted lines - locations of flight lines of the 1971-72 and 1975 surveys. Heavy dots - locations of initial explosives, fuel, and food caches.

Figure 3. After page 2. (a) Surface elevation, (b) bedrock elevation, and (c) ice thickness maps of East Antarctica in the vicinity of the traverse. Maps were contoured from digitized values of elevation and ice thickness collected by the NSF/SPRI/TUD airborne radio echo-sounding surveys in 1971-72 and 1975 (D. Vaughan, SPRI, written comm., 1993). Heavy line - location of traverse.

Figure 4. After page 4. (a) Profile of elevation along the traverse. (b) Surface elevation map of East Antarctica in the vicinity of the traverse from airborne radio echo-sounding (see Figure 2 for details). Contour interval $-100 \mathrm{~m}$.

Figure 5. After page 5. Example of a seismic field record from a seismic shot at $\mathrm{km}$ 74.7, after application of a $500 \mathrm{~ms}$ automatic gain control. The vertical axis represents two-way travel time, in ms. The horizontal axis is shot-receiver offset, in $\mathrm{m}$.

Figure 6. After page 5. A portion of the stacked and binned multichannel seismic data along the traverse.

Figure 7. After page 6. Observed gravity (in milligal) along the traverse.

Figure 8. After page 6. Raw magnetic measurements (in nannoTesla) along the traverse line.

Figure 9. After page 6. A - An example of a typical trace (no processing applied). This trace was recorded in surface location $\mathrm{km} 67.4$ with $4 \mu \mathrm{sec}$ pre-trigger length, $20 \mathrm{mV} / \mathrm{div}$ gain and $0.08 \mu \mathrm{sec}$ sample interval using the four-stage transistor avalanche source and the Fluke 97 based recorder. B - $96 \mathrm{~km}$ long section, acquired from Taylor Dome (surface location $\mathrm{km}-10$ ), on the

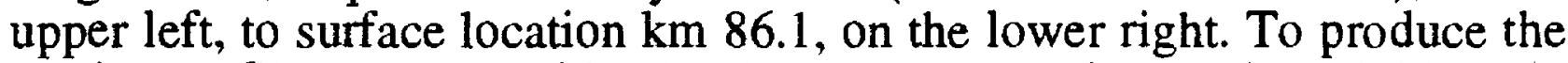
section, 8 files were combined, adjacent traces subtracted, variable gain applied and traces plotted by surface location. The gap in the image occurs where no data were acquired.

Figure 10. After page 7. Schematic diagram showing the different working groups and their spatial relationship.

Figure 11. After page 8. Wind speed, surface temperature, and wind direction plotted as a function of time for the main party and the advance party. Upper left 
plot shows location of both parties along the traverse line as a function of time.

Figure 12. After page 8. Comparison between barometric pressure (in milliBar) as a function of time measured by the roving station (P2) vs. that measured by the temporary base camp. Base camp measurements were carried out every 5 minutes and were averaged by taking the median over a 6 hour period.

Figure A3.1 After page A3.1 Illustration (not to scale) of the ground-penetratingradar set-up during acquisition. See text for detailed description.

Figure A3.2 After page A3.2 Diagrams describing the components of the ground penetrating radar system. Arrows indicate the directions of signal transmission, dashed lines describe DC power supply, thick lines connect the acquisition receiver assemblage components, and thin lines connect the receiver triggering assemblage components.

Figure A5.1 After page A5.5 Top - barometric pressure (in milliBar) measured along the traverse line. Bottom - elevation along the traverse (similar to Figure 4). Note that pressure is plotted increasing downward to facilitate comparison with the elevation. 


\section{SUMMARY}

The East Antarctic Seismic Traverse (EAST93) was a geophysical traverse designed to image the bedrock under the East Antarctic ice cap. The traverse started $10 \mathrm{~km}$ west of the Taylor Dome drill site and $25 \mathrm{~km}$ west of the exposed bedrock of the Transantarctic Mountains at Lashly Mt. and ended $323 \mathrm{~km}$ west of the drill site over the Wilkes subglacial basin (Fig. 1). The traverse was located subparallel to latitude $78^{\circ} \mathrm{S}$ starting $30-50 \mathrm{~km}$ north of the Victoria Land Traverse (1958-1959). It was carried out jointly by the U.S. Geological Survey and Stanford University, U.S.A., together with the Institute of Geological and Nuclear Sciences, and Victoria University, New Zealand, during December 1993 and January 1994. The geophysical traverse included $236 \mathrm{~km}$ of multichannel seismic reflection data at $150 \mathrm{~m}$ shot intervals, $312.5 \mathrm{~km}$ of gravity data collected at intervals of 2.1 $\mathrm{km}, 312.5 \mathrm{~km}$ of magnetic data (total field intensity) collected at average intervals of 0.5 $\mathrm{km}$, and $205 \mathrm{~km}$ of ground penetrating radar at intervals of $77 \mathrm{~m}$. Relative locations and elevations of the entire traverse were measured at intervals of $150 \mathrm{~m}$ by traditional surveying methods, and tied to three absolute locations measured by the Global Positioning System (GPS).

EAST93 is the first large-scale geophysical traverse on the polar plateau to our knowledge since the early 1960s. As such, the experiment presented several logistical challenges: (1) how to collect regional seismic profiles during the short Antarctic summer; (2) how to keep the scientific instruments running with minimal protection in harsh conditions; and (3) how to combine daily moves of camp with full days of work. The scientific and logistical aspects of the project proceeded, in general, according to plan despite the harsh conditions and our lack of previous experience on the polar plateau. Two unanticipated problems affected the progress of the work: the strong wind which slowed seismic acquisition, and the break-down of one of the large traverse vehicles. The major operational lessons of this project are. (1) Primacord laid close to the surface is not an adequate seismic source for imaging under the thick East Antarctic ice sheet, despite positive prior tests on the Ross Ice Shelf. (2) It is necessary to reduce the 6-7 hours spent daily on camp move and other chores by integrating the living quarters into the working teams, and by improving vehicle warming methods and generator housing.

The following report details the operational and logistical aspects of the work, the weather and ground conditions, the technical aspects of acquisition of geophysical data, and lessons and recommendations for future geophysical traverses. 


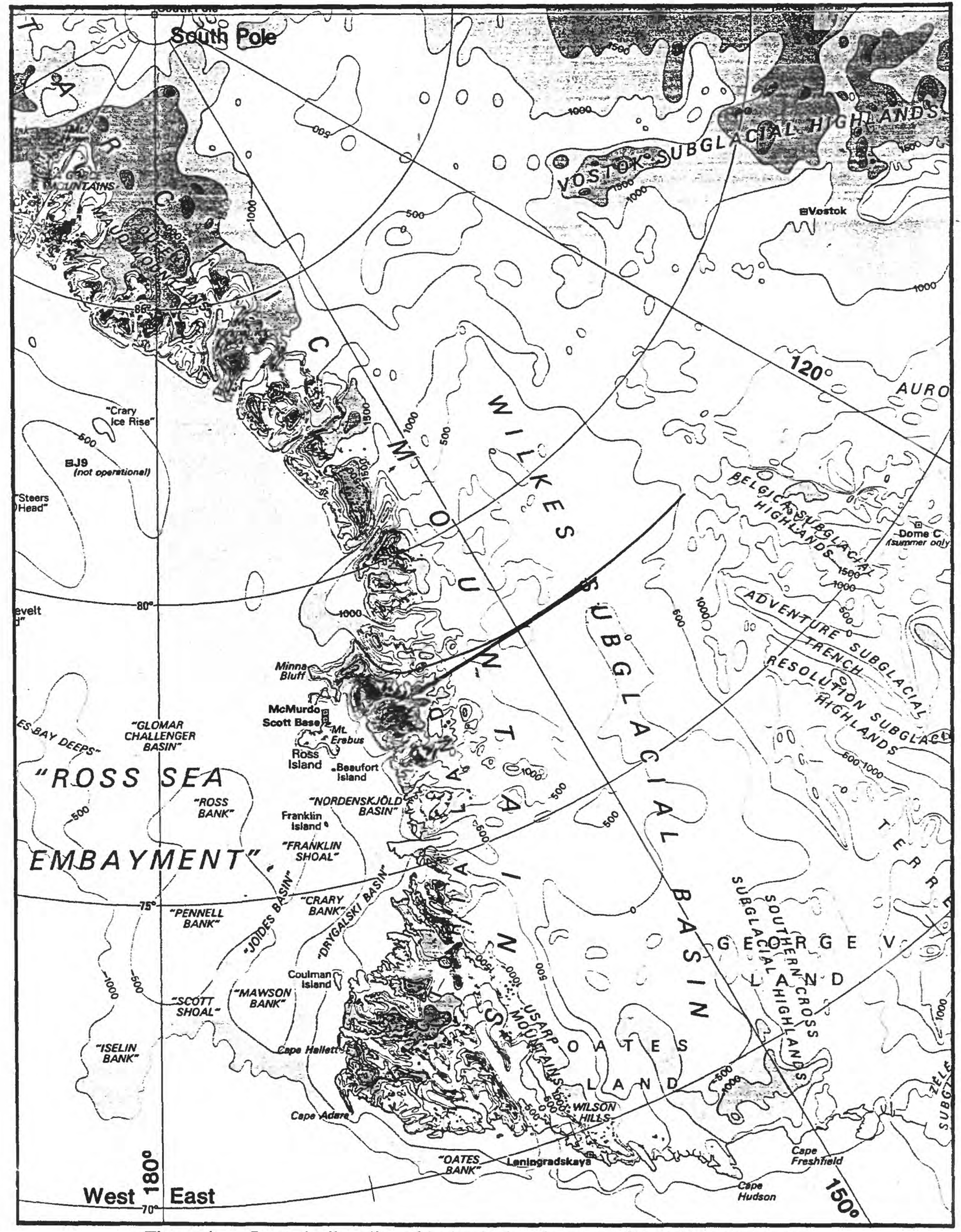

Figure 1. Isostatically-adjusted bedrock elevation map of East Antarctica next to the Transantarctic Mountains (from Drewry, 1983). The location of the traverse is shown by the heavy line, and the location of the $1958 / 59$ traverse is shown by the light line. 


\title{
SCIENTIFIC OBJECTIVES
}

By imaging the bedrock under the East Antarctic ice sheet between the Transantarctic mountains and the Wilkes subglacial basin, we hoped the experiment would enable us

(1) To provide quantitative constraints for modeling the uplift of the Transantarctic Mountains and the subsidence of the Wilkes Basin as a flexed lithospheric plate with a freeedge at the boundary with West Antarctica (Stern and ten Brink, 1989).

(2) To map the extent of the Ferrar dolerite sills and basalts inland from the mountains in order to discern whether they originated from an active mantle plume or from rifting and passive upwelling (Elliot, 1991).

(3) To analyze the seismic stratigraphy of the Wilkes Basin sediments to help resolve the debate about the climatic conditions and the size of the ice sheet in the Cenozoic, in particular, whether parts of East Antarctica were deglaciated for much of the Late Cenozoic (Webb, 1991).

\section{PREVIOUS WORK IN THE AREA}

Only one traverse was carried out in this area prior to the EAST93 experiment: the Victoria Land Traverse led by A.P. Crary in 1958-1959 (Crary, 1963) (Fig. 1). Crary's traverse collected gravity and seismic reflection and refraction data, but the quality of the data were limited by the technology available at the time. Ice coring and related glaciological studies were carried out at Taylor Dome between 1991-1994, located $10 \mathrm{~km}$ east of the start of the line (Fig. 2) (Grootes and Steig, 1992; Morse and Waddington, 1992). Rock outcrops at Beacon Heights, located $45 \mathrm{~km}$ east of the start of the line, were mapped by McElroy and Rose (1987), and laboratory measurements of velocity and density of rocks from the Beacon Group in this area of the Transantarctic Mountains were made by Barrett and Froggatt (1978). A regional airborne radio-echo sounding survey grid, centered at about km 0 (Fig. 3), was carried out in January 1975 by the joint National Science FoundationScott Polar Research Institute-Technical University of Denmark (NSF-SPRI-TUD) program (Drewry, 1982). Several flight tracks carried out in 1971-72 as part of the continent-wide airborne radio-echo sounding survey by the NSF-SPRI-TUD program (Drewry, 1983) crossed the traverse area (Fig. 3). Ice thickness and surface elevation data, digitized by the British Antarctic Survey (BAS) personnel (David Vaughan. pers. comm.. 1993) from film records collected by these two projects, were used to construct the base map for our experiment (Fig. 3). Estimated errors in these airborne surveys were $<5 \mathrm{~km}$ in navigation, $1 \%$ in ice velocity, $26 \mathrm{~m}$ in air and $14 \mathrm{~m}$ in ice in signal resolution, and 50$150 \mathrm{~m}$ in flight height (Drewry et al., 1982).

\section{FIELD PERSONNEL}

\author{
United States \\ Uri ten Brink \\ (USGS Woods Hole) - Co-leader \\ Rafael Katzman \\ (MIT/WHOI Joint program in Oceanography) - Student \\ Yizhaq Makovsky \\ (Stanford University) - Student \\ New Zealand \\ Stephen Bannister \\ Mike Collins \\ Ron Hackney \\ (Inst. Geological and Nuclear Sciences) - Co-leader and shooter \\ (New Zealand Antarctic Program) - Mechanic \\ Jan de Vries \\ (Victoria University, Wellington) - Student \\ (New Zealand Antarctic Program) - Search And Rescue (SAR)
}




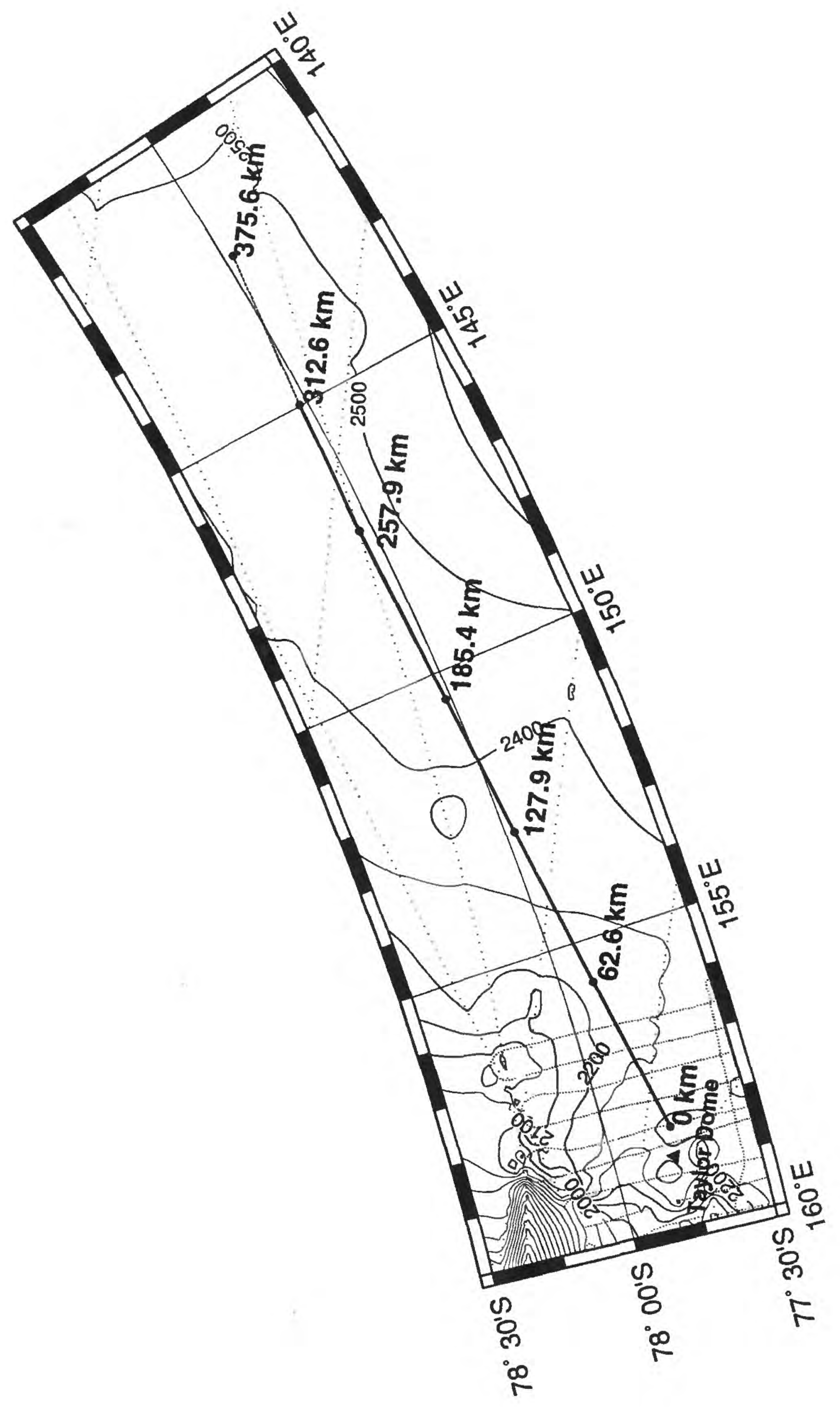

敢宅. 层웡 获焉 는 .

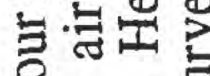
药市 ㅇํำำ 튼 靣氙㤩 密 㐘艺 氙帚合 의 प号它 管

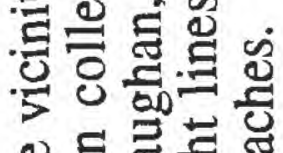

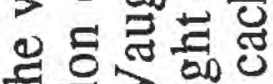
$\Rightarrow$ 잉

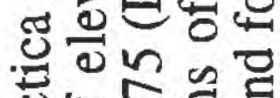

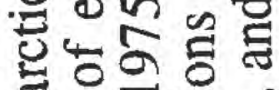
是呵 可 苗 प्रे क्षे ㅇ: 2 . : 紐

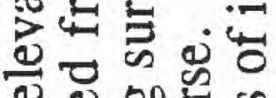

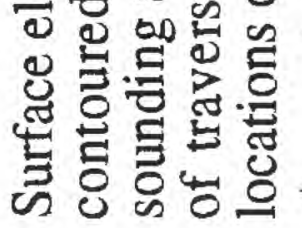
i

苟 

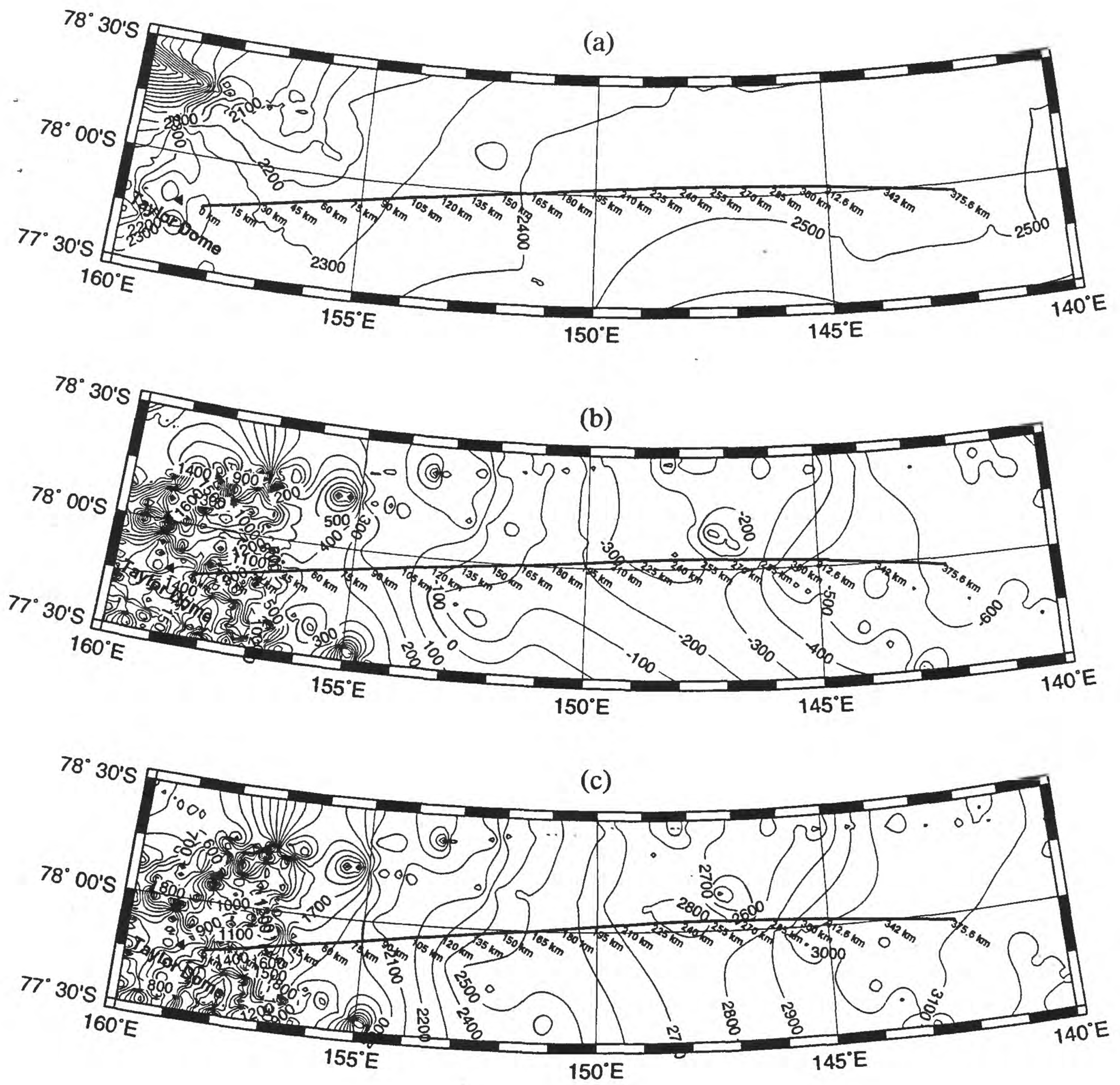

Figure 3. (a) Surface elevation, (b) bedrock elevation, and (c) ice thickness maps of East Antarctica in the vicinity of the traverse. Maps were contoured from digitized values of elevation and ice thickness collected by the NSF/SPRI/TUD airborne radio echo-sounding surveys in 1971-72 and 1975 (D. Vaughan, SPRI, written comm., 1993). Heavy line - location of traverse. 
David King

Bill King

John West
(Inst. Geological and Nuclear Sciences) - Electronic technician

(New Zealand Antarctic Program) - SAR person

(Dept. Surveying \& Land Information) - Surveyor

\section{TIME TABLE AND LOG}

The party spent a total of 54 days in the field, with some personnel arriving in the field 2 days earlier and leaving a day later than the rest of the party. Of these 54 days, 20 days were spent shooting seismics, 17 days were lost to bad weather, 4 days were lost to dealing with the broken large traverse vehicle (Tucker 069) and consequently the need to rearrange the work, 3 days were lost to emptying supply caches and getting air supply, 5 days were spent in organizing the work and overcoming technical problems, and 2 days were spent in preparation for pullout (including a runway for C-130).

22 Nov. -2 Dec: Twin-Otter support for reconnaissance of traverse, fuel and food dispersal in depots at $\mathrm{km} 0$ (Alpha), $\mathrm{km} 63$ (Delta), $\mathrm{km} 128$ (Bravo), km 185 (Echo), km 258 (Charlie), and km 313 (Foxtrot) (Fig. 2), pickup of parachutes used to drop explosives, and GPS survey of reference points at $\mathrm{km} 0,128$, and 258 .

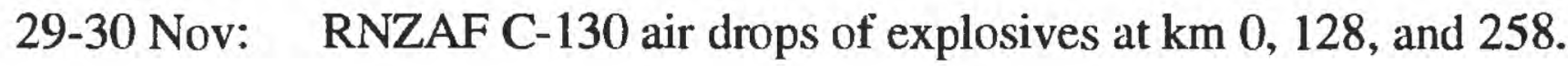

1 Dec: Put-in of survey party at Taylor Dome.

3-6 Dec: Put-in of main party and its equipment at Taylor Dome.

8-10 Dec: $\quad$ Main party moves to km 0 (Alpha), repairs damage to snow streamer, refuels from Taylor Dome.

11 Dec: Seismic work begins, fuel supply and generator replacement by Twin-Otter.

15 Dec: Work terminates at 3 p.m. due to high winds.

16 Dec: No work due to high winds.

18 Dec: $\quad$ No work due to a snow storm.

22-23 Dec: 2 people travel $165 \mathrm{~km}$ overland to bring more explosives from Alpha and replacement generators and transformer from Taylor Dome. They measure a continuous radar profile.

23-26 Dec: No work due to high winds.

27 Dec: $\quad$ High winds, advance without shooting seismics between $\mathrm{km}$ 75-90.

29-31 Dec: No work due to high winds.

4 Jan: Walk-away shots, Makovsky develops tooth abscess and needs to be evacuated.

$6 \mathrm{Jan}$ High winds, advance without shooting seismics between $\mathrm{km}$ 162-185.

7 Jan: $\quad$ Survey team finishes flagging to $\mathrm{km} 375$. 
8 Jan: $\quad$ Slow movement due to bad sastrugi (parallel ridges of wind-blown hard snow). Shot size and length increased by $50 \%$.

9 Jan: $\quad$ Survey team joins main team at $\mathrm{km} 200$.

10 Jan: $\quad$ Skidoo AL3 breaks front shaft and is towed on a sled until the end of the traverse.

12 Jan: Twin-Otter makes a difficult landing due to sastrugi. It evacuates surveyor, electronic technician, and Makovsky, and brings replacement computer hard drive.

13 Jan: Morning whiteout prevents advance through sastrugi field. Work commences in afternoon, but Tucker 069 (which tows the camp) develops transmission problem.

14 Jan: $\quad$ Runway prepared for Twin-Otter to bring replacement for transmission.

15 Jan: $\quad$ No work due to whiteout.

16 Jan: Main camp splits. Tucker 069 and mechanic left behind, science party continues.

17-19 Jan: No work due to bad storm.

20 Jan: Digging out and work. Twin-Otter with mechanic and Makovsky arrives at Tucker 069 location to replace transmission. Replaced transmission fails.

21 Jan: $\quad$ Twin-Otter arrives at Tucker 069 location with a mechanic and another transmission. Science party retrieves explosives, fuel and food from depot Charlie.

22-23 Jan: Tucker 069 and science party meet and move toward Foxtrot, but forced to stop at $\mathrm{km} 270$ after complete transmission failure of Tucker 069.

24-25 Jan: Gravity and magnetics measured out to $\mathrm{km} \mathrm{312.6.} \mathrm{Supplies} \mathrm{at} \mathrm{depot}$ Foxtrot retrograded to $\mathrm{km} \mathrm{270.} \mathrm{Twin-Otter} \mathrm{arrives} \mathrm{with} \mathrm{snow} \mathrm{groomer} \mathrm{and}$ operator to prepare runway for LC- 130 .

26 Jan: $\quad$ Last day of seismic work.

28-29 Jan: $\quad$ Pull-out of equipment and personnel by 7 LC-130 flights from $\mathrm{km} 270$.

\section{INSTRUMENTATION}

\section{Location And Surveying}

USGS/NMD determined GPS positions at the fuel depots near km 0 (Alpha), 127.95 (Bravo) and 257.86 (Charlie) (Fig. 2). GPS surveys were conducted on November 29 and December 1, 1993. Geoid separations were obtained from OSU91A Geoid Interpolation Program, The Ohio State University, department of Geodetic Science and Surveying. Horizontal accuracy is in the 1-meter range. 

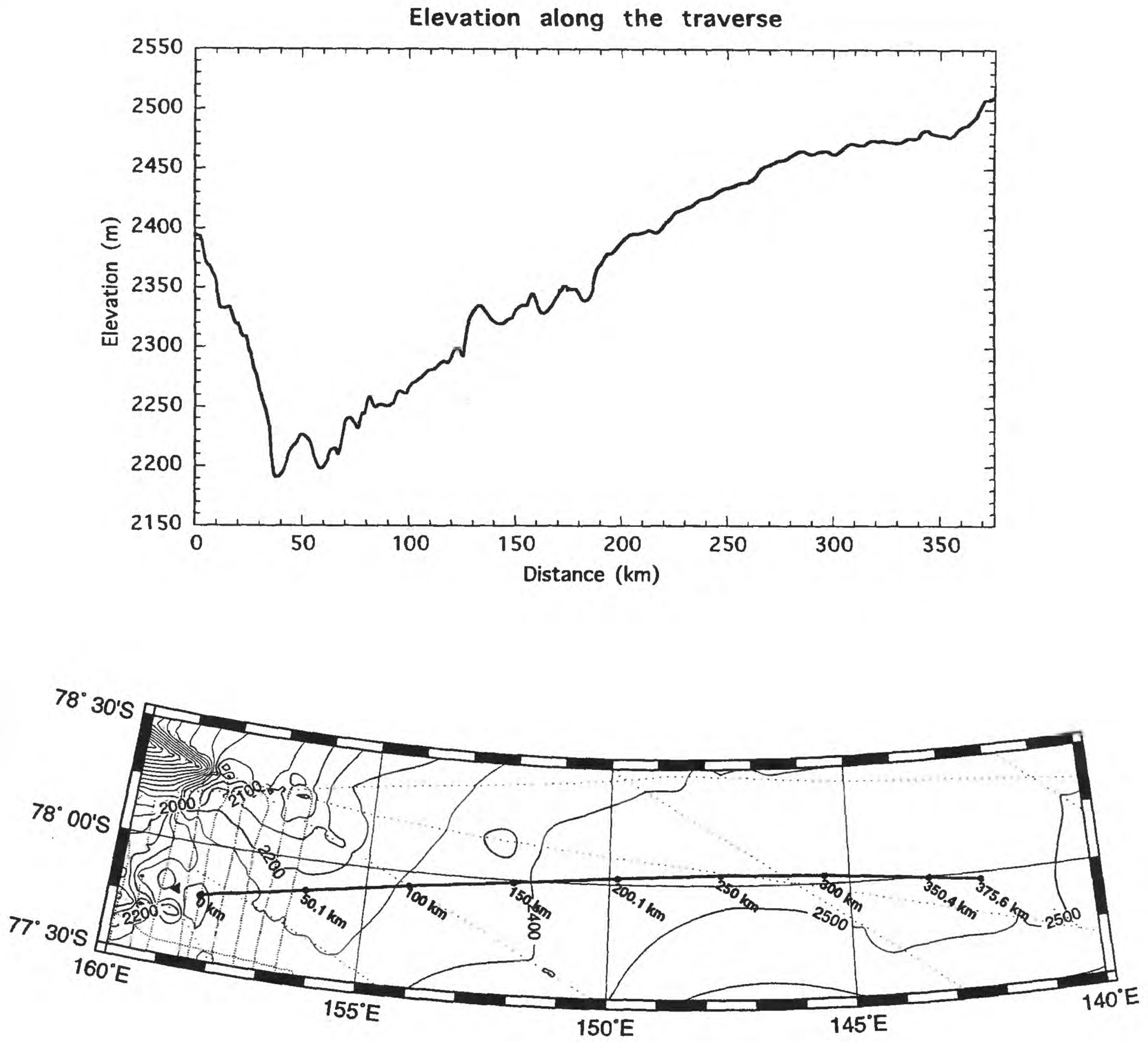

Figure 4. (a) Profile of elevation along the traverse. (b) Surface elevation map of East Antarctica in the vicinity of the traverse from airborne radio echo-sounding (see Figure 2 for details). Contour interval $-100 \mathrm{~m}$. 
In addition to these measurements, relative elevation measurements were carried out by the DOSLI surveyor, John West, in the advance survey group using conventional land surveying techniques. The location of each measured position along the traverse starting from $\mathrm{km} 0$ is given in Appendix 1 with elevations calculated using the Alpha GPS site as the absolute elevation and accepting a geoidal separation of $50.1 \mathrm{~m}$ there (see Table below). (The distance azimuth from Alpha to ' $\mathrm{km} 0^{\prime}$ were $937.5 \mathrm{~m}$ and $229.82^{\circ}$.) The (relative) ground measurement survey agreed with the GPS values at Bravo and Charlie within to 3 meters, about the order of error that might be expected using the GPS in this manner.

\section{GPS POINTS:}

\begin{tabular}{|l|l|l|l|l|l|}
\hline Site & $\begin{array}{l}\text { WGS } 84 \\
\text { Coordinates }\end{array}$ & $\begin{array}{l}\text { Elliptical } \\
\text { Height }(\mathrm{m})\end{array}$ & $\begin{array}{l}\text { Geoid } \\
\text { Separation }(\mathrm{m})\end{array}$ & $\begin{array}{l}\text { Ortho. Height } \\
(\mathrm{m})\end{array}$ & $\begin{array}{l}\text { Surveyed } \\
\text { Height }(\mathrm{m})\end{array}$ \\
\hline & & & & & \\
\hline Alpha & $\begin{array}{l}77^{\circ} 46^{\prime} 42.29^{\prime \prime} \mathrm{S} \\
158^{\circ} 20^{\prime} 30.89^{\prime \prime} \mathrm{E}\end{array}$ & 2345.5 & -50.1 & 2395.6 & $\begin{array}{l}2395.6 \\
\text { (Accepted as } \\
\text { origin) }\end{array}$ \\
\hline Alpha AZ & $77^{\circ} 47^{\prime} 14.01^{\prime \prime} \mathrm{S}$ & 2342.7 & -50.1 & 2392.8 & \\
\hline & $158^{\circ} 21^{\prime} 21.31^{\prime \prime} \mathrm{E}$ & & & & 2324.1 \\
\hline Bravo & $77^{\circ} 57^{\prime} 08.15^{\prime \prime} \mathrm{S}$ & 2270.6 & -53.7 & 2324.3 & \\
\hline & $152^{\circ} 55^{\prime} 04.87^{\prime \prime} \mathrm{E}$ & & & & 2440.9 \\
\hline Charlie & $78^{\circ} 05^{\prime} 23.16^{\prime \prime} \mathrm{S}$ & 2388.9 & -54.1 & 2443.0 & \\
\hline & $147^{\circ} 20^{\prime} 28.87^{\prime \prime} \mathrm{E}$ & & & & \\
\hline
\end{tabular}

Figure 4a shows a profile of the elevation measured along the traverse and Fig. 4b shows the location of the traverse, with approximate elevation contours from the 1970 s airborne data. Appendix 1 lists the locations of surveyed positions, and their elevations.

Additionally, barometer readings were taken during supply flights to 6 points along the traverse and at intervals of $2.1 \mathrm{~km}$ along the traverse, but these were not used to calculate elevation.

\section{Seismics}

The shot parameters were as follows:

Type of source - Geoflex 40 detonating cord (primacord) with $40 \mathrm{~g} / \mathrm{m}$ dynamite (200 grain).

Manufacturer - ICI Australia.

Shot size - $\quad 1.6 \mathrm{~kg}$ (40 m long cord) between $\mathrm{km} \mathrm{0-162.}$

$2.4 \mathrm{~kg}$ (60 m long cord) was used between $\mathrm{km} \mathrm{185-270.}$

Shot depth - $16.5 \mathrm{~cm}\left(6.5^{\prime}\right)$.

Shot interval - $150 \mathrm{~m}$.

Shot-receiver offset for near trace - $175 \mathrm{~m}$ between $\mathrm{km} \mathrm{0-162.}$

$185 \mathrm{~m}$ between $\mathrm{km} \mathrm{185-270.}$

Survey geometry -Marine, except for walk-away calibration shots to a stationary streamer (maximum offset of $3.2 \mathrm{~km}$ ) at $\mathrm{km} 148$ and 270. 
Figure 5. Example of a seismic field record from a seismic shot at $\mathrm{km} 74.7$, after application of a $500 \mathrm{~ms}$ automatic gain control. The vertical axis represents two-way travel time, in ms. The horizontal axis is shot-receiver offset, in $\mathrm{m}$.

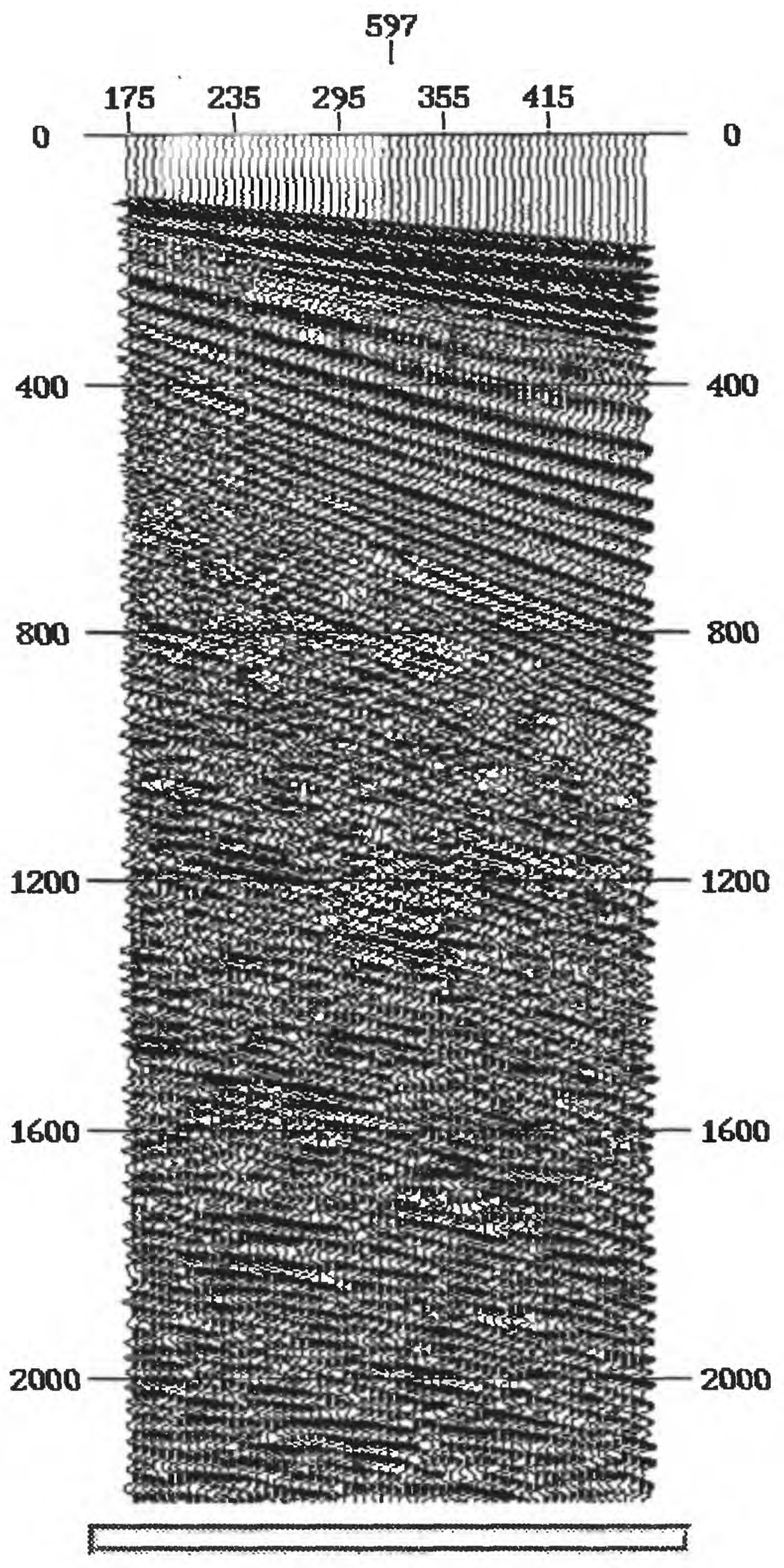




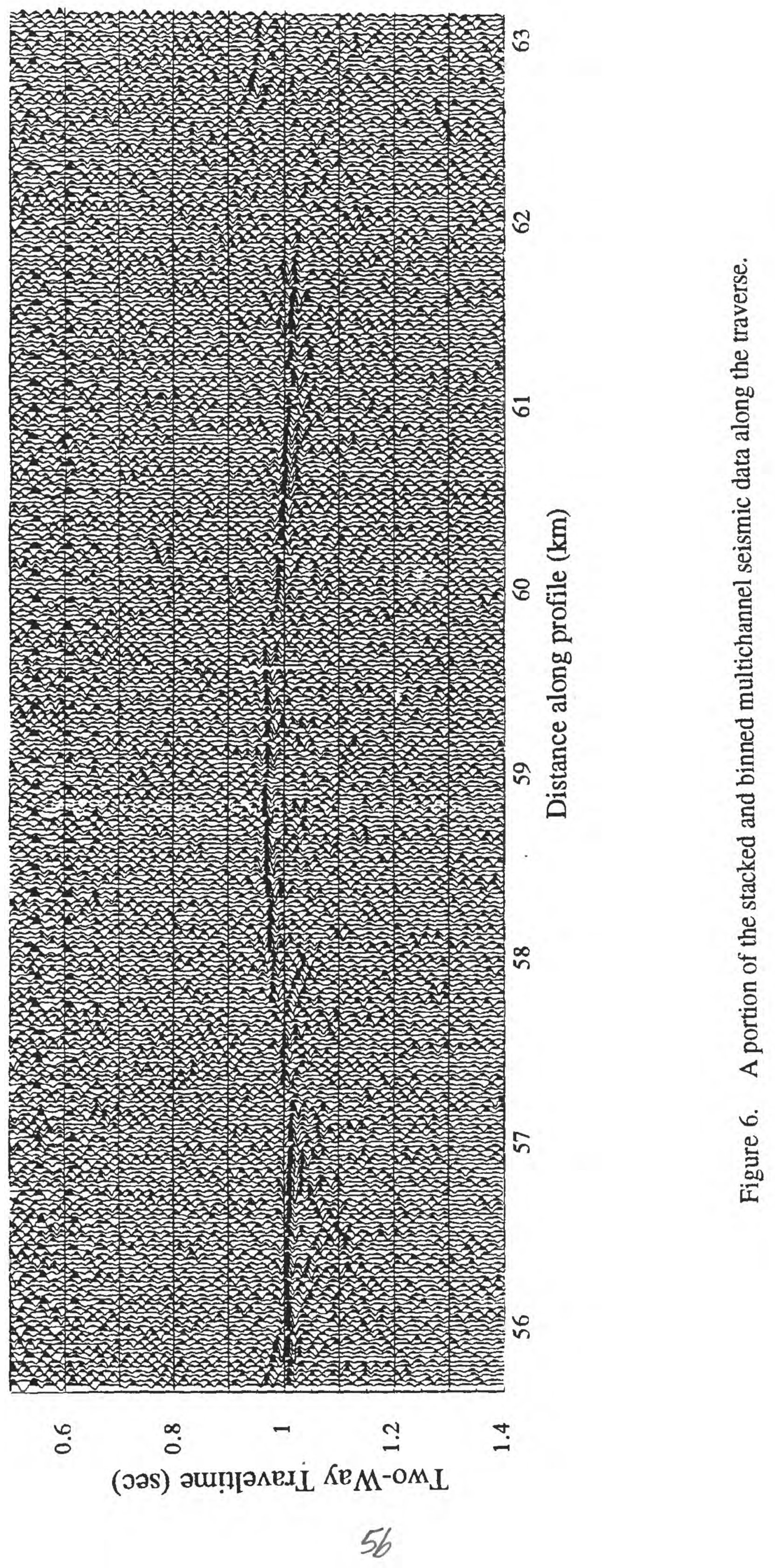


Seismic data were recorded using a Geometrics Strataview R-60, a 486 PC-based portable computer, with 18 bit A/D converter, $500 \mathrm{MByte}$ hard drive, Colorado Jumbo tape drive and a built-in plotter. Sampling rate was $1 \mathrm{msec}$ and record length was $4 \mathrm{sec}$. The unit demultiplexed as it recorded and had several display functions. Processing for field quality control was performed using the Eavesdropper ${ }^{\mathrm{TM}}$ software developed by the Geological Survey of Kansas.

The seismic receiving system consisted of a 300 -m-long snow streamer made by NorskHydro of Norway. The streamer is a towed Kevlar seismic cable with 60 take-outs (channels) at $5 \mathrm{~m}$ intervals. Each take-out is a cable with 4 single-axis gimbaled geophones per channel, $\sim 3.1 \mathrm{~m}$ apart.

Fig 5 shows a field record of seismic data collected during the experiment, and Fig. 6 shows a portion of the stacked data. The location of shot points is listed in Appendix 1.

\section{Gravity}

Gravity observations were made every $2.1 \mathrm{~km}$, using a LaCoste-Romberg gravimeter G179. The gravimeter was continuously attached to a battery that supplied power to an internal heater, which maintained the gravimeter at a constant temperature. In addition, the gravimeter was cased in a Perspex box with holes for the dials, and this box and the battery were kept in a wooden box. The gravimeter was kept inside a vehicle at all times and was brought outside only for the measurements. The gravimeter values were tied to Scott Base gravity station before the experiment. Instrument drift was calculated by successive measurements over several days at the same location $(\mathrm{km} \mathrm{269.7)} \mathrm{The} \mathrm{observed} \mathrm{gravity} \mathrm{is}$ plotted in Fig. 7 and listed in Appendix 2.

\section{Magnetics}

Magnetic readings were carried out using two Geometrics G-856 magnetometers. One magnetometer was used as the roving station at intervals of $300-450 \mathrm{~m}$ and the second one as a temporary base station at the daily base camp to record the diurnal variations in the magnetic field. The base station magnetic sensor and magnetometer required adequate insulation to operate properly at these temperatures. The sensor was wrapped with insulated material and a hand warmer was wedged between the sensor and the insulation. The magnetometer was enclosed in a food box containing a hot water bottle. The raw magnetometer readings observed by the 'roving' station are plotted in Fig. 8 and listed in Appendix 1 together with their day of measurement.

\section{Ground penetrating radar}

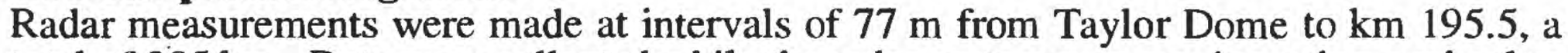
total of $205 \mathrm{~km}$. Data were collected while the radar system was moving using a wheel to measure the distance and an electroinc trigger to start acquisition. The system included low frequency receiving and transmitting antennae $(1.25 \mathrm{MHz})$ with resistivity constants of 400 Ohm. The radar transmitter supplied a voltage pulse of $750 \mathrm{~V}$. The data were received and sampled using a digital oscilloscope (Fluke 97 Scopemeter) which stacked 256 readings to form one trace. Data were sampled for 40 microseconds $(\mu \mathrm{sec})$ at $0.08 \mu \mathrm{sec}$ per sample and recorded on a BCC Avanti 486 portable computer. The data were converted into SEG-Y format which allowed us to use standard seismic processing tools for display and analysis. Detailed description of the acquisition system and data reduction are given in Appendix 3.

The trace in Figure 9A displays the characteristic waveform of the radar signal. The first arriving air-wave is clipped to allow good resolution of the reflected signal. Reflections from within the ice around 7-8 $\mu \mathrm{sec}$ are difficult to discern on this single trace. The signal arriving around $10 \mu \mathrm{sec}$ is an artifact we can not explain. The ice-rock interface reflection can be clearly seen at $13 \mu \mathrm{sec}$ two-way-time translating to a depth of about $1 \mathrm{~km}$. We plan 


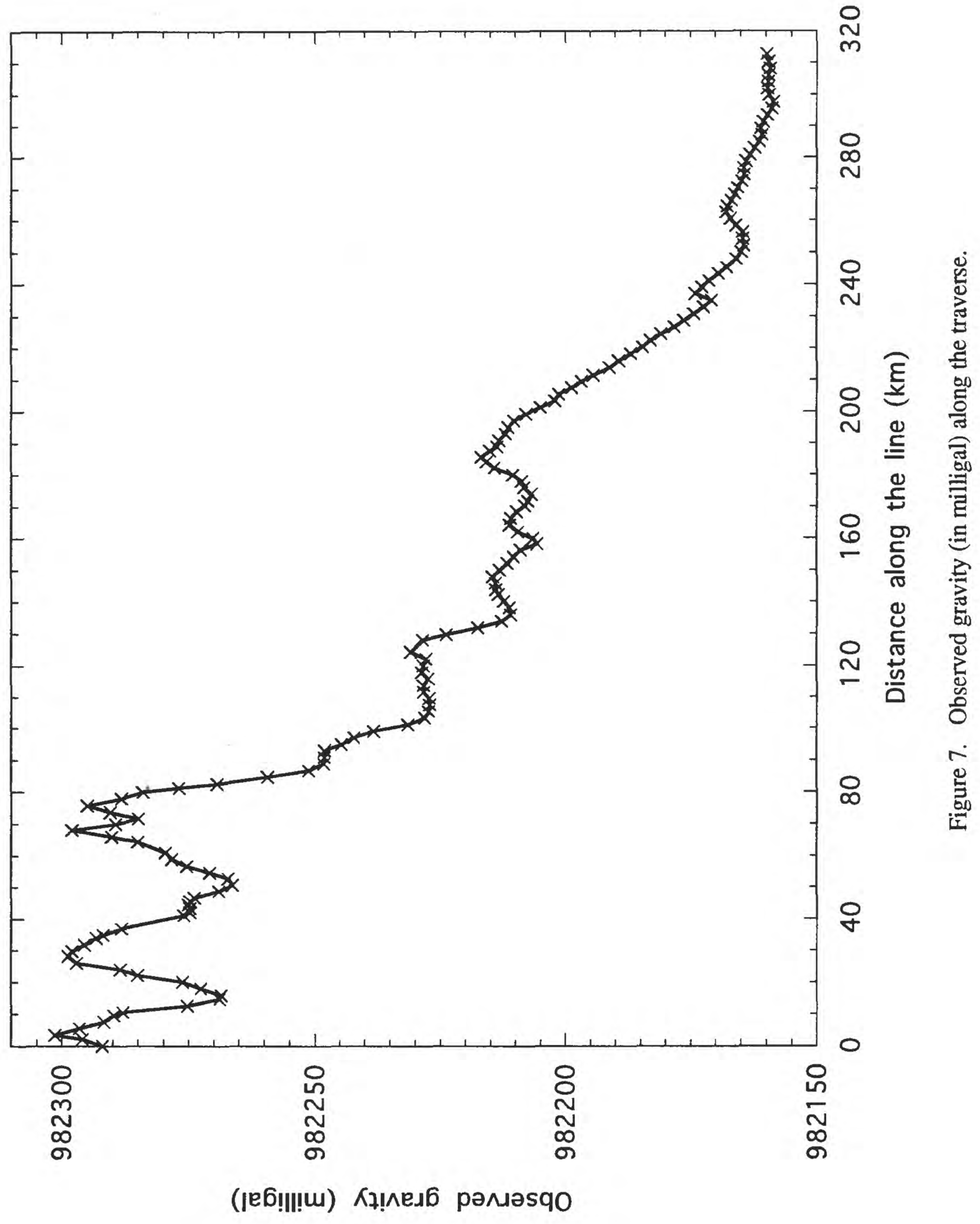




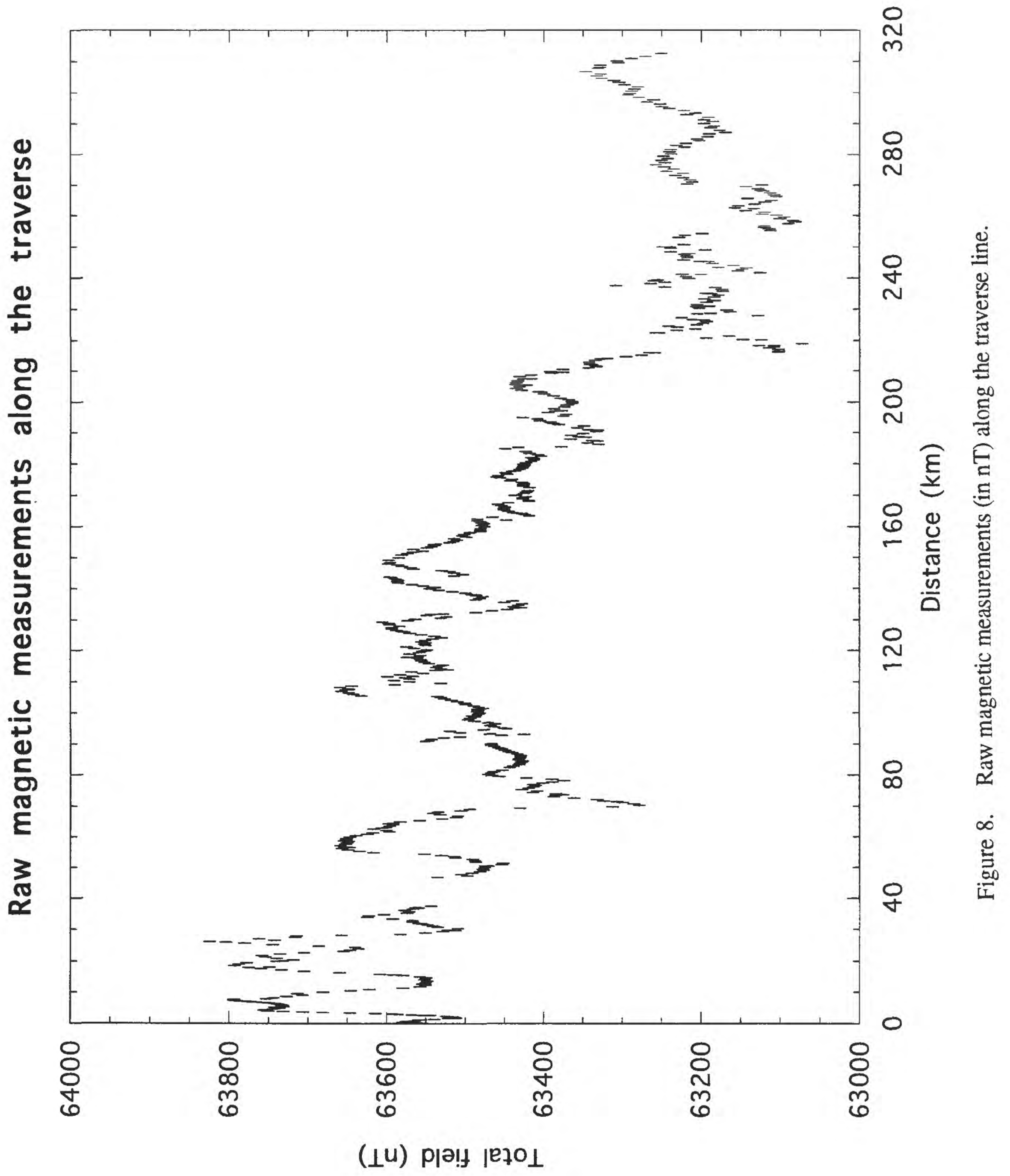




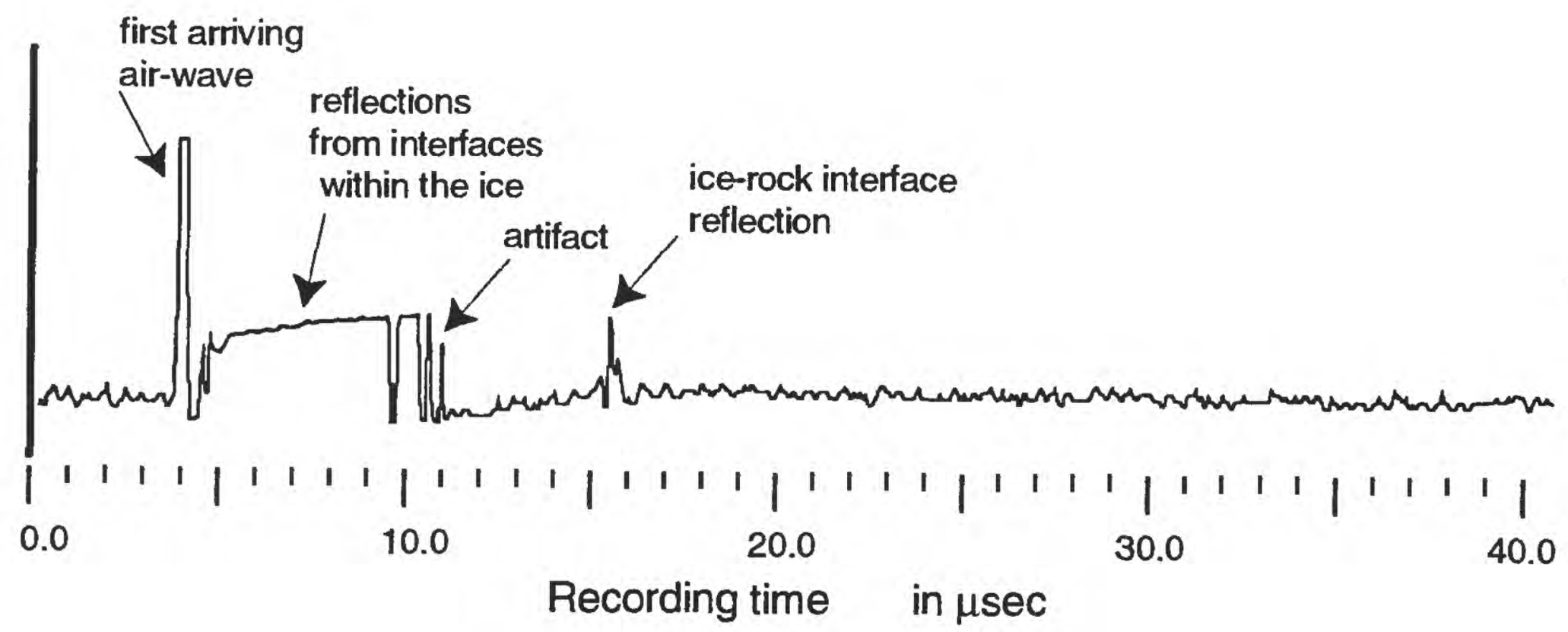

Figure 9a. An example of a typical trace (no processing applied). This trace was recorded in surface location $\mathrm{km} 67.4$ with $4 \mu \mathrm{sec}$ pre-trigger length, $20 \mathrm{mV} / \mathrm{div}$ gain and $0.08 \mu \mathrm{sec}$ sample interval using the four-stage transistor avalanche source and the Fluke 97 based recorder. 


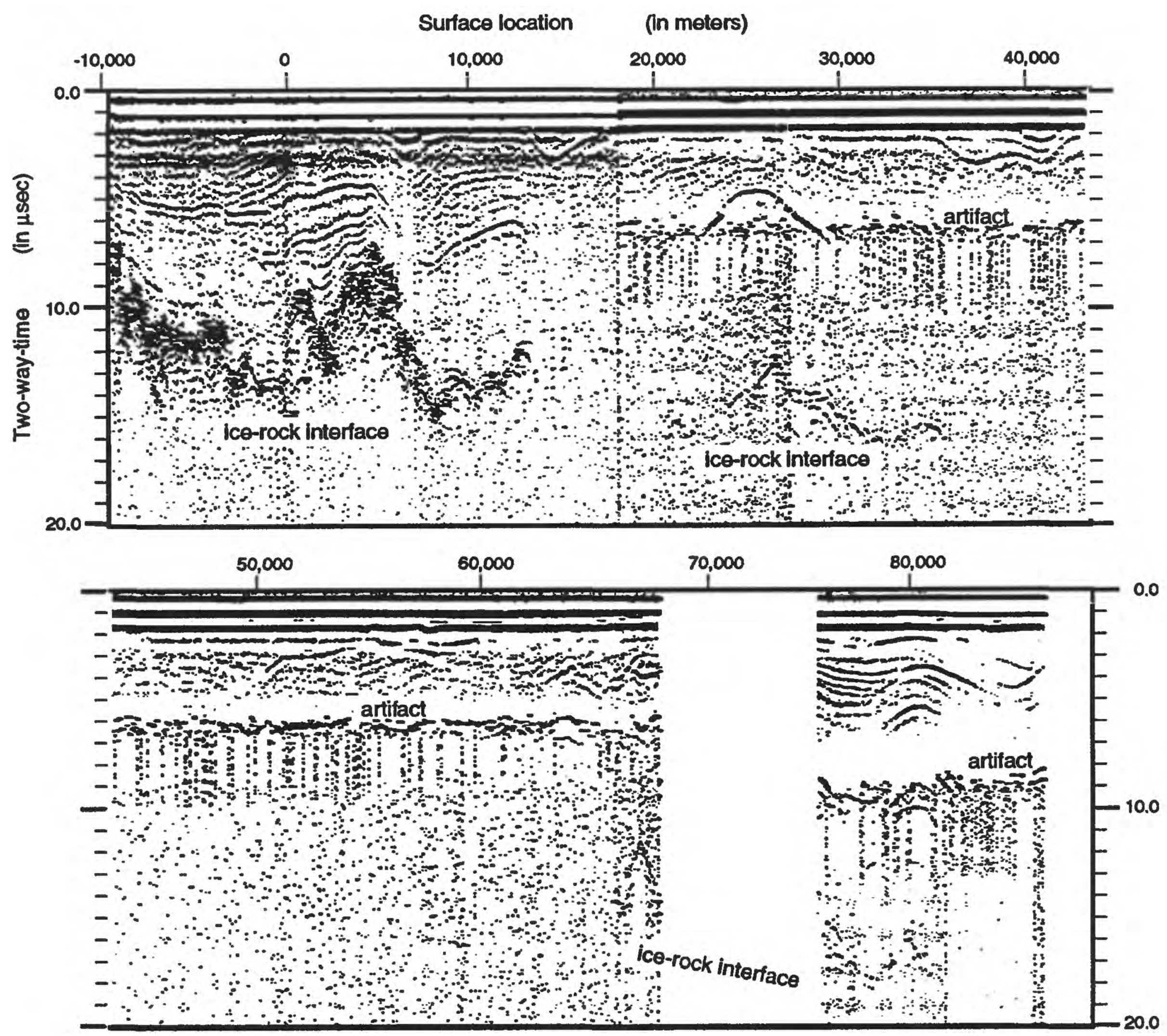

Figure 9b. $96 \mathrm{~km}$-long section, acquired from Taylor Dome (surface location $\mathrm{km} \mathrm{-10),} \mathrm{on} \mathrm{the} \mathrm{upper} \mathrm{left,} \mathrm{to} \mathrm{surface} \mathrm{location} \mathrm{km} 86.1$, on the lower right. To produce the section, 8 files were combined, adjacent traces subtracted, variable gain applied and traces plotted by surface location. The gap in the image occurs where no data were acquired. 

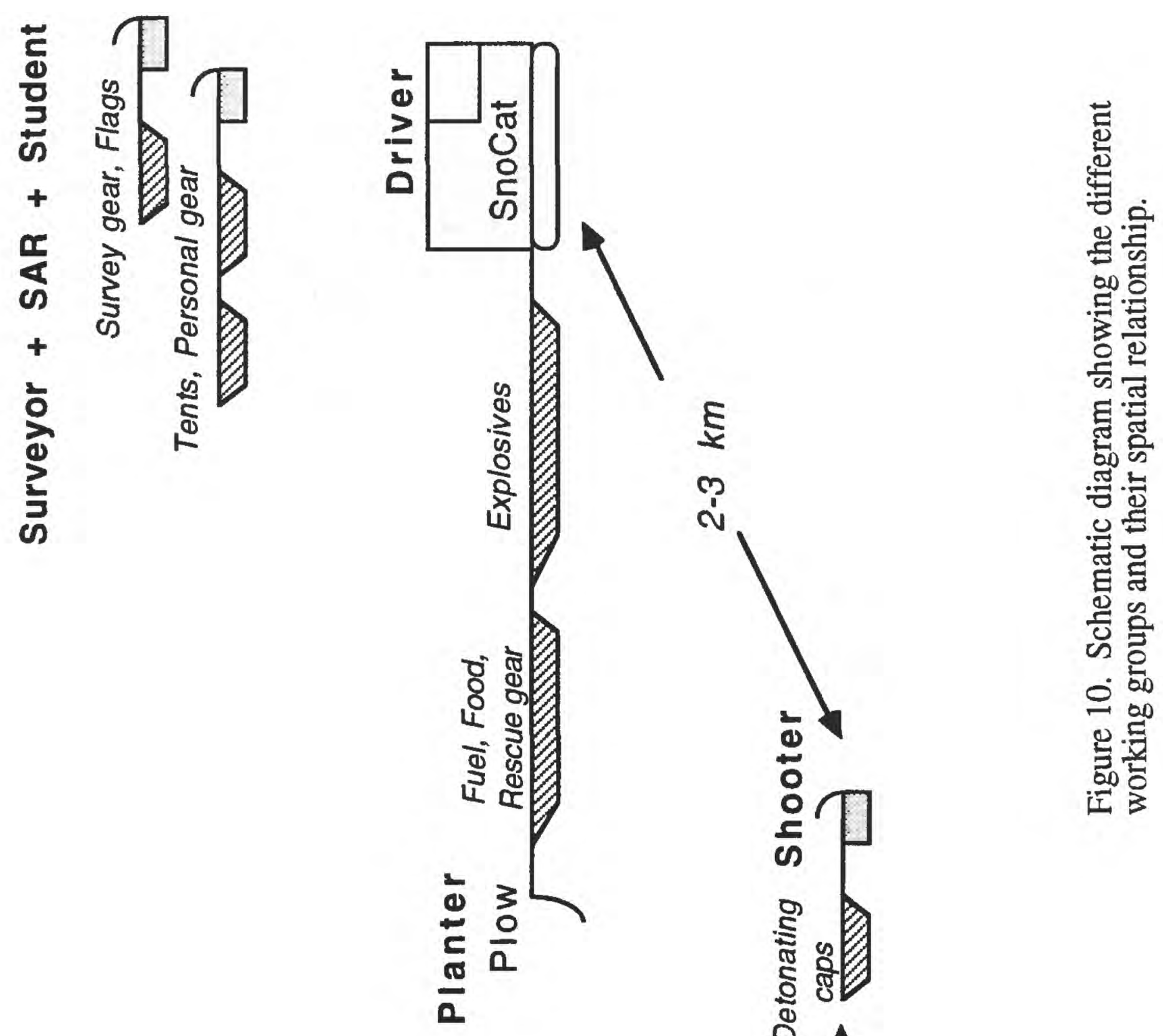

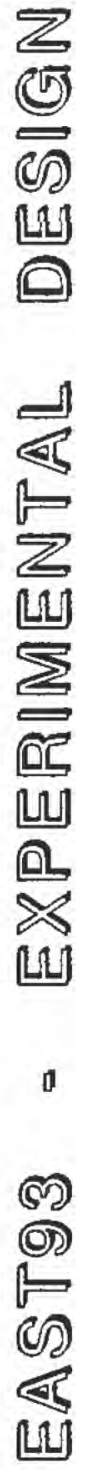
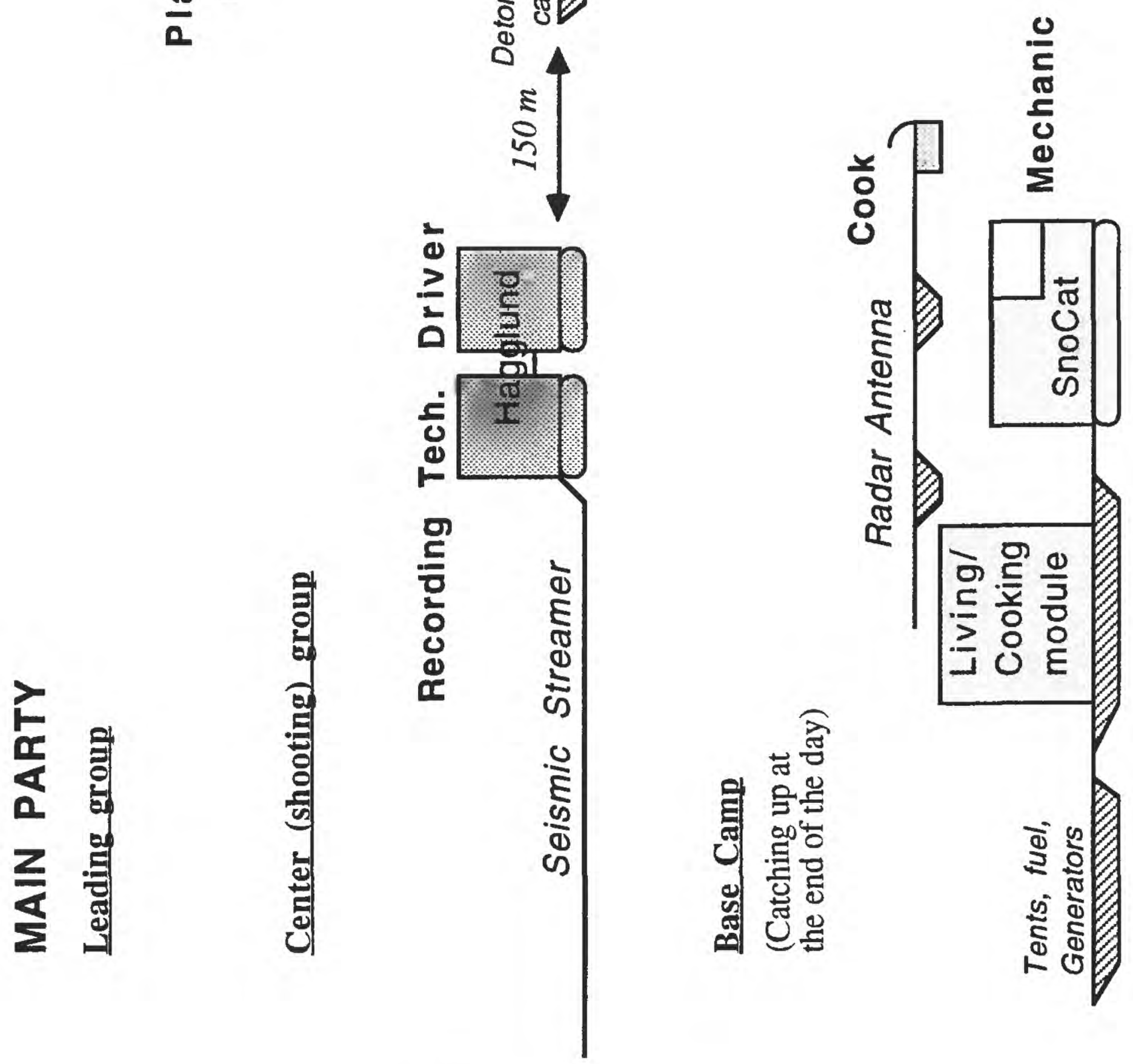
to subtract adjacent traces from each other in each data-file to cancel the air wave and most of the artifacts, and to enhance the reflected signals.

The image in Figure 9B displays a $96 \mathrm{~km}$ long section, acquired across the flank of the Transantarctic mountains to the edge of Wilkes basin. A strong reflection from the ice-rock interface can be seen around 7-8 $\mu \mathrm{sec}$ on the left of the image. The rough shape of this reflector describes a rough subglacial topography similar to the exposed topography of the Transantarctic mountains. This reflector becomes discontinuous and weak as the travel time increases to about $17 \mu \mathrm{sec}$, or about $1400 \mathrm{~m}$ deep, and disappears completely on the right of the image.

The features seen between the surface and the ice-rock interface are reflections of interfaces within the ice. These can be different layers of ice deposited on the ice sheet, layer of dust etc. These reflectors seem to wrap around the topographic features at the base of the ice in complex flow patterns. The horizontal bands of signal around $10 \mu \mathrm{sec}$ are the artifacts pointed out in Figure 9A.

\section{METHODOLOGY}

The field work was conducted in the following way (Fig. 10):

An advance survey group which included a professional surveyor with an Electronic Distance Meter (EDM) (J. West), a Search And Rescue (SAR) person (B. King), and a student (R. Katzman). The advance group moved autonomously 100-150 km ahead of the main party on 2 skidoos with Nansen/Tamworth sleds. The survey party was put in the field 6 days before the main party. It advanced, on average, $15 \mathrm{~km}$ a day. It surveyed and planted flags every $150 \mathrm{~m}$. It drew food and fuel from caches.

The main party consisted of 3 separate working groups, which converged nightly:

1. The leading group laid 40-60 m long pieces of detonating cord below the surface using a plow at $150 \mathrm{~m}$ intervals, and carried out magnetic and gravity measurements. The group included 2 people: The Tucker driver, who was also a qualified SAR person (J. de Vries), checked for crevasses, measured the magnetic field every 450-600 meters, and wind speed, temperature and barometric pressure every $2.1 \mathrm{~km}$. The second person (U. ten Brink/R. Hackney) on foot behind the snow plow held the end of the detonating cord while the Tucker pulled the plow forward, stomped on the trench to pack the snow over the detonating cord, and cut the end of the detonating cord at the proper length. The second person also measured gravity every $2.1 \mathrm{~km}$. The leading Tucker stopped and idled its engine before each shot detonation, in order to reduce the ambient noise on the seismic records.

The leading group had the following equipment: A Tucker Snocat which towed (i) an Anare sled (a midsize sled-1770 lb., 12 x $6 \mathrm{ft}$., 2-3 ton carrying capacity) loaded with explosives and 3 fuel drums, (ii) a Maudheim sled loaded with heavy rescue gear (400 lb.), spare streamer sections, spare generators, 460 liter drums and miscellaneous equipment as necessary, and (iii) a snow plow.

2. The center (shooting) group consisted of a large tracked vehicle (Hagglund) towing the 60 channel, $300 \mathrm{~m}$ long snow streamer, and an Alpine-II Skidoo towing a box sled ("Sleepy sledge") loaded with detonators. The seismic recording unit was housed in the back of the Hagglund. It included 3 people : the shooter (S. Bannister / U. ten Brink) on a skidoo about $150 \mathrm{~m}$ in front of the Hagglund, the driver of the Hagglund, and the recording 

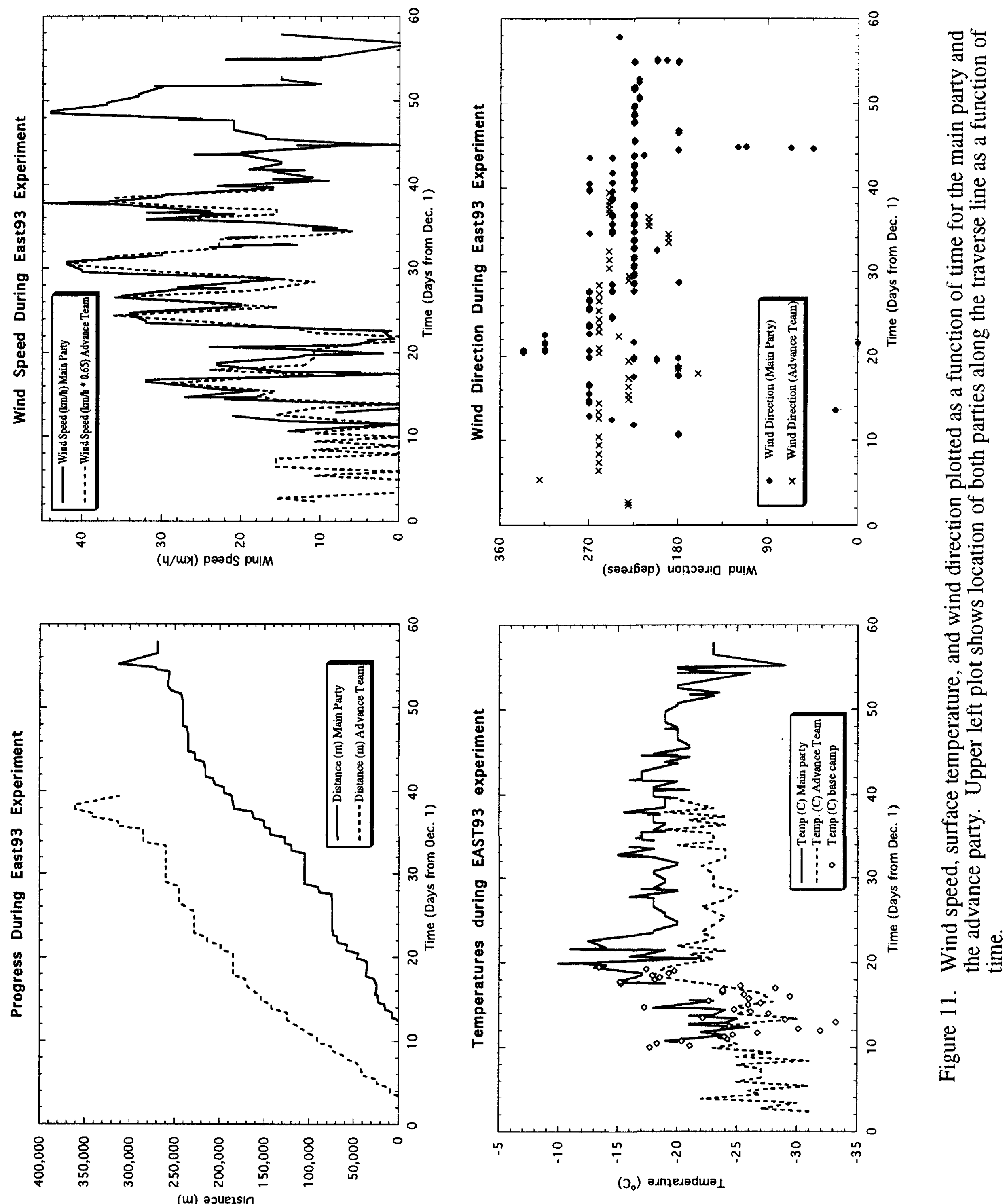

(w) aJue1s!a

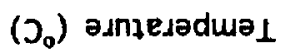


technician in the back of the Hagglund. At each shot location the shooter connected a detonator to the primacord (laid by the leading group), and the shot wire to the detonator. The Hagglund driver (R. Hackney / S. Bannister) connected the end of a $180 \mathrm{~m}$ long electric wire trailing behind the shooter into the Hagglund, turned the flag nearest to him upside down (to reduce wind noise during recording), idled the engine for 2 minutes, and then shut the engine off. The driver was also responsible for alerting the leading and camp groups of an impeding shot and giving the 'all clear' signal. The recording technician (D. King / R. Katzman) in the back of the Hagglund carried out the seismic recording and the quality control. The center (shooting) group followed $2-3 \mathrm{~km}$ behind the leading group by starting work 1-1.5 hours after the leading group. The distance between the groups was maintained to minimize the effect of Tucker engine noise on the seismic records.

3. This camp group included a mechanic (M. Collins) and a cook (Y. Makovsky), who also took care of the radar measurements. The camp group was also responsible for setting up magnetic and barometric base stations, the data from which was later compared to the measurements from the roving magnetometer station. Finally, the camp skidoo driver (Y. Makovsky) was responsible for turning the flags back to right side up. The camp group started moving about 2-3 hours before the end of the shooting day. Earlier movement would interfer with the shooting. radar data were recorded continuously during this movement.

The base camp had the following equipment: (i) a Tucker Snocat towing the camp (an Anare sled with a living module (Wannagan), and a 1-ton sled with 6 fuel drums, Herman Nelson, 2 generators, tents, and miscellaneous camp equipment), and (ii) an Alpine II skidoo towing the radar equipment (radar antennae and Nansen and banana sleds on which separate receiving and transmitting equipment were emplaced).

\section{ENVIRONMENTAL CONDITIONS}

The elevation above mean sea level was $2395 \mathrm{~m}$ at $\mathrm{km} 0$ (10 km west of McMurdo Dome drill site), decreasing to $2190 \mathrm{~m}$ at $\mathrm{km} 37.8$ and increasing gradually to $2470 \mathrm{~m}$ at $\mathrm{km}$ 312.6 with undulations of up $20 \mathrm{~m}$ on a $10 \mathrm{~km}$ scale (Fig. 4 and Appendix 1).

The snow condition varied from soft to steel-hard, with rapid changes in space and time (Appendix 4). The snow was often hard enough to support human steps and skidoo tracks without breaking through. The majority of the terrain was smooth enough to minimize delays in traveling. Some rough stretches with sastrugi up to $1.5 \mathrm{~m}$ were encountered. These stretches obviously slowed our progress. Rough stretches were often seen to correspond with lows in the topography (particularly noticeable from $123.6-126.3 \mathrm{~km}$ ). This effect could be accounted for by the fact that such lows would channel cold air, resulting in regions of higher wind speed and greater potential to form sastrugi. In general, the smoothest terrain was encountered at the start of the line and also at the very end of the line. The sastrugi were consistently in a SW - NE orientation parallel to the wind direction and were prominent between $\mathrm{km} 122$ and 265 (Appendix 4).

Temperatures ranged between $-15^{\circ} \mathrm{C}$ and $-33^{\circ} \mathrm{C}$, with the warmest period between December 17 and January 15 . The maximum temperature registered was $-10^{\circ} \mathrm{C}$ (Appendix 5 and Fig. 11)

Generally, the sky was clear with persistent katabatic wind from the west to southwest at $10-40 \mathrm{~km} / \mathrm{h}$ (6-22 knots), and snow blowing at wind speeds above $30 \mathrm{~km} / \mathrm{h}$ (17 knots). The daily maximum wind occurred around 6 p.m. local time. Wind speed may have been positively correlated with good weather in McMurdo sound and was highest between December 15 and January 19. Occasionally, weather fronts from the south passed through, 


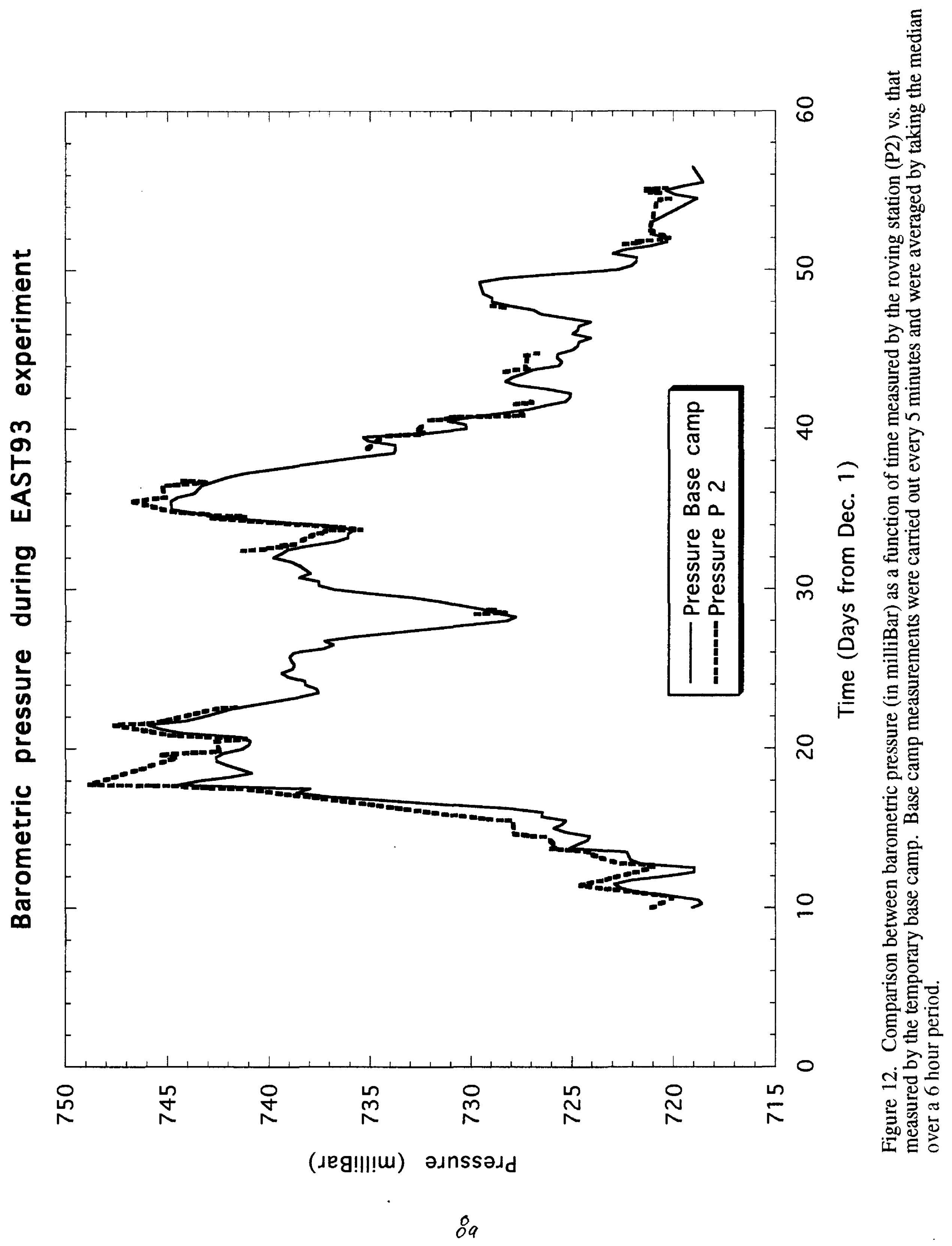


with warmer temperatures, little wind, snow fall, and whiteout conditions. Comparison between the survey group and the main group up to $150 \mathrm{~km}$ apart along the line indicated uniform weather conditions along the line (Appendix 6 and Fig. 11).

Figure 11 shows the wind speed, surface temperature and wind direction plotted as a function of time for both the advance party and the leading and camp groups of the main party. Fig. 12 shows the barometric pressure during the experiment measured by the leading group at roving stations every $2.1 \mathrm{~km}$ along the traverse and by the base camp at daily camp sites. Three separate measurements by two different barometers were carried out at each roving station. The average values of the three measurements for each instrument (P1 and $\mathrm{P} 2$ ) are given in Appedix 5. Figure A5.1 compares the barometric pressure measured by instrument P1 to the measured elevation along the traverse. Note the pressure drop around $\mathrm{km} 150$ (Day 39) which was unrelated to temperature or elevation. Base camp measurements of pressure and temperature were carried out automatically every 5 minutes. Their median values over 6 hours intervals are given in Appendix 7.

\section{VEHICLES AND MAINTENANCE}

Appendix 8 details the report from the NZAP mechanic, Mike Collins, on the mechanical aspects of the traverse.

\section{LESSONS AND RECOMMENDATIONS}

\section{Seismic Work}

1. Detonating cord as a seismic source: Detonating cord (also known as Primacord) appears to be the only source that could be prepared and laid at a rate comparable to the rate of shooting. From experience in the SERIS experiment (ten Brink et al., 1993), 2-3 drilling crews of 2 each and 2 additional people for shot preparation are needed to achieve a similar acquisition rate with down hole shots. In addition, comparisons between downhole and surface primacord shots carried out during the 1990/91 SERIS experiment indicated that the source signature from the surface primacord shot was superior to that from a downhole source (Melhuish et al.,1993).

The seismic signal returned from subglacial layers was, however, disappointingly low relative to ambient noise and "ground roll". Bottom could be clearly imaged in the first 75 $\mathrm{km}$ of the seismic work where the ice is relatively thin $(700-1700 \mathrm{~m})$, but could seldom be imaged at depths of $2000-3000 \mathrm{~m}$. It appears that the high near-surface velocity gradient in the ice generates strong turning waves and surface waves at the expense of more deeplypenetrating energy. However, this must only be a partial explanation in light of our positive test results with primacord as a seismic source during the SERIS experiment where the near-surface velocity gradient is equally high. High firn noise following shooting, which was a frequent complaint during the IGY experiments $(\mathrm{C}$. Bentley and J. Behrendt, pers. comm., 1993), did not pose a problem in the frequencies of interest $(<80 \mathrm{~Hz})$.

2. Acquisition rate: The leading group laid 10-12 shots an hour, while also carrying out gravity, magnetic, and weather measurements. The center (shooting) group fired up to 10 shots an hour. Generally, 60-80 shots were fired in a full working day with a maximum of 108 shots in one day. These acquisition rates were anticipated on the basis of our experience at the SERIS experiment (ten Brink et al., 1993) and proved reasonable. There were no major problems related to the seismic work in the field.

3. Seismic acquisition at high wind speeds: Shooting could not be carried out at wind speeds in excess of $30-35 \mathrm{~km} / \mathrm{h}$ for the following reasons. (a) Wind noise on the streamer 
became high. (b) Blowing snow at these wind speeds could potentially shorten the electric circuit and cause premature detonation. (c) Detonating required tying electric wires with exposed fingers, and fingers became unbearably cold due to wind chill (temperatures with wind chill were $-50^{\circ} \mathrm{C}$ to $-70^{\circ} \mathrm{C}$ ), (d) The plastic cover of the shooting wires became brittle exposing the copper wires and causing misfires. (e) Regular electric tape used for attaching the detonator and for fixing broken shooting wire, was non-adhesive at these temperatures.

4. Snow plow: The snow plow performed very well. The use of the plow over surface-laid primacord had several advantages: (a) It saved one person ( 2 people were originally allocated, one at each end of the primacord). (b) A buried primacord produced negligible air wave energy. (c) The explosion of buried primacord left much less visible residue (i.e. pollution) than either a surface-laid primacord, or a downhole explosion (as seen during our 1990/91 SERIS experiment). In fact, the explosion of buried primacord was so clean that it was difficult to spot the exact location of the explosion after 24 hours. Plowing could be performed in almost any weather condition.

5. Snow streamer: The use of the Norwegian snow streamer to receive the seismic data (instead of conventional geophones) considerably reduced the logistical effort and manpower in planting geophones and rolling cables.

6. Modern recording system: The use of the Geometrics Strataview recording system (leased at the last moment) instead of the DFS-V system, proved crucial. Its flexibility and ease of use meant that work could continue even after the departure of the electronic technician on January 12 . In addition, its battery power consumption was $1 / 3$ that of the DFS-V and its processing and noise display capabilities allowed us to make operational judgments based on the seismic results.

\section{Effect On Humans}

1. Length of work day: The daily shift of camp and the low temperatures meant that working days became very long (16-24 hours). Warming up the vehicles in the mornings and preparation for work took 3 hours from wake-up time. Likewise, setting up camp in the evenings, dinner, downloading and saving data, etc., took additional 3 hours. In addition, shooting started 1-1.5 hours after the leading group started plowing and stopped 1 hour before the base camp caught up with the leading and shooting team. In the beginning of the experiment, the leading group would lay an extra $2 \mathrm{~km}$ for the shooting group to detonate at the start of the next day, thus eliminating 1-1.5 hours waiting period for the shooting team. This arrangement was abandoned after several storms completely buried the exposed ends of the primacord. Subsequently, all laid primacord was detonated the same day. No better solution was found for the wasted time connected with the base camp. The camp group could not be sent ahead of the other groups because we could not anticipate our daily progress.

2. Living quarters: It is useful for field parties larger than 4 people to have a meeting room for planning and dividing the daily work. Our living quarters served this purpose in addition to serving as a central kitchen and galley. The Wannagan used in this traverse for living quarters was, however, too heavy, rigid, and cumbersome for a traverse, and could only be safely loaded and unloaded off the sled with a forklift. It is recommended that a light-weight structure with a collapsible roof (like that found in some RVs) be designed. The structure should be permanently attached to skis and should fit into a C-130. Alternatively, the field party should be divided to several independently-moving teams of 24 people sleeping and cooking in tents. This second option would make the management of the project difficult, and would result in redundancy of field equipment. 
3. Other lessons: Apart from the advance survey team, the only two people working outside and unprotected from the wind were the person behind the plow and the shooter.

Oxygen deprivation and carbon monoxide poisoning did not occur during the experiment.

\section{Logistics}

1. Caches: A major problem of any traverse is planning the caches. Caches were placed in advance of the experiment by a Twin-Otter aircraft with quantities based upon a certain rate of daily progress. However, when these rates were not met due to bad weather, technical, or mechanical problems, it was necessary to shuttle tens of $\mathrm{km}$ (24-60 hours round-trip) to either bring more fuel and food or to clear unused caches. The only alternative is to have Twin-Otter support on demand. However, this alternative can also result in delays if the twin-otter cannot fly due to weather or mechanical problems. In general, cache planning for EAST93 was good, except for the first cache (at the beginning of the line) where we spent longer than anticipated assembling and preparing the equipment for work.

2. Explosive airdrop; Airdrop of explosives by RNZAF C-130 flying from Christchurch en-route to McMurdo was highly successful. Explosive palettes landed within $50 \mathrm{~m}$ of the caches. Explosives were not damaged and did not pose any risk for later usage. Removal of parachutes and palettes by Twin-Otter immediately after air drop was helpful.

3. Other lessons: Air support by both VXE-6 C-130 and the Twin-Otter aircrafts and coordination by USAP were timely, efficient, and professional.

Communication via MacOps was excellent and response to logistical requests over the radio was timely.

\section{Mechanical Aspects}

1. Generators and batteries: NZAP supplied diesel generators ( 2.5 to $5 \mathrm{~kW})$ that ran on JP8. Three of the 4 generators failed for a variety of reasons. In addition, their output in the high altitude of the plateau was $40 \%$ of that expected at sea level. All generators quit working or were shut down to avoid damage during storms involving blowing snow. It is recommended that only Mogas generators be used and that they should be housed in a portable protective shack. Alternative energy sources should be explored, because the plateau is generally sunny and has steady winds. Mixing $230 \mathrm{~V}$ and $110 \mathrm{~V}$ power supplies (due to the joint US/NZ nature of the event) was inconvenient. A thought should be given to connecting the seismic recording system directly to the Hagglund battery to save on battery charging, because the seismic recorder is the largest consumer of battery power.

2. Starting vehicle engines: Starting the Tucker vehicles in the cold temperatures and winds prevailing on the plateau was a lengthy and an energy-consuming process. This was not the case with the Hagglund whose engine is completely enclosed. The construction of a transparent (Plexiglas) shelter to protect and passively warm the Tucker engine is recommended. Alternatively, a chemically-based heater (akin to hand warmers) should be supplied to be put on the engine block overnight.

3. Fuel consumption: Fuel consumption for all vehicles was on average 1.5 times greater than at sea level (for exact numbers see Appendix 8).

4. Sleds: The Maudheim and 1-Ton sleds were ideally suited for the traverse. Their size and their carrying weight are small enough to handle crevasse and sastrugi areas. They can be lifted and dragged by several people. Most important, they are low enough to the ground that fuel drums and other heavy loads can be dragged onto them. Their major disadvantage is that their frames are not strong enough to hook several of them in a row. The lack of 
strong frames limits the possibility of having a bulldozer tow a train of several Maudheims. The larger Anare sled was strong enough to tow other sleds behind it but was too high off the ground to load fuel drums, a broken skidoo, etc.

\section{Rate of progress}

1. The daily speed of traverse without carrying out seismic work was only $2-3$ times faster than a traverse while carrying out seismic work. All large vehicles traveled only in first gear because of the surface conditions and the load they were pulling. Sastrugi were encountered along $60 \%$ of the traverse, some of them up to $1.5 \mathrm{~m}$ high. The sastrugi did not cause any particular problems except to slow vehicle movement $(1-2 \mathrm{~km} / \mathrm{h}$ instead of 3-4 $\mathrm{km} / \mathrm{h}$ for the large vehicles). We opted not to move in areas of sastrugi when ground definition was poor, to avoid damage to vehicles.

\section{ACKNOWLEDGMENTS:}

We thank the following people for their contributions to the success of the experiment: Dale Benson and Larry Hotham, USGS, Reston, for accurately locating reference points along our line, Ed Waddington and Dave Morse, University of Washington, for sharing their design of the radar acquisition system and the driving software, Steve Hodge, USGS, Tacoma, for introducing us to the art of radar acquisition and loaning his portable radar, Eivind Rygg and Norsk-Hydro for lending the snow streamer, Rob Huggins and Geometrics for supplying the seismic recording system, Bill Robinson, USGS, Menlo Park, for fixing and preparing an alternative DFS-V system, and John Behrendt, USGS, Denver, for loaning the alternative gravimeter. We thank the following people at McMurdo Station for their support during the experiment: Dave Bresnahan and Simon Stephenson for their timely response to our needs, Rick Campbell for organizing the deep field air support, Bill Danforth, camp manager of Taylor Dome, for his help and hospitality, Steve Kottmeier and Tom George for their help with scientific and computer equipment, Dale Willett and Billy Stranger for their help with the Tucker's transmission, and Billy Stranger and Buck Tilley for their help in the field.

We thank Scott Base personnel for their hospitality and help in mobilizing and demobilizing the experiment, especially the stores personnel and mechanics, Gus McAlister, Jeremy Ridgen, and Grant West. We also thank the New Zealand Air Force for carrying out precision airdrops of explosives at the caches. Funding was obtained from the Foundation of Research Science and Technology (NZ) and the National Science Foundation (US).

Rebecca Drury helped with the prepation of the report and Rebecca Drury and Ann Swift offered many constructive comments to an earlier version of the report.

\section{REFERENCES}

Barrett, P.J. and Froggatt, P.C.,1978, Densities, porosities, and seismic velocities of some rocks from Victoria Land, Antarctica, N.Z. J. Geol. Geophys., 21, p. 175-187.

Crary, A.P. 1963. Results of United States Traverses in East Antarctica, 1958-1961. IGY Glaciological Report, Number 7, December 1963. World Data Center A : Glaciology, American Geographical Society, New York, 35 p.

Drewry, D.J., 1982, Ice flow, bedrock, and geothermal studies from radio echo-sounding inland of McMurdo Sound, Antarctica, in: C. Craddock (Ed.), Antarctic Geoscience, Madison, University of Wisconsin Press, p. 977-983. 
Drewry, D.J., Jordan, S.R., and Jankowski, E., 1982, Measured properties of the Antarctic ice sheet: Surface configuration, ice thickness, volume, and bedrock characteristics, Ann. Glaciol. 3, p. 83-91.

Drewry, D.J. (ed.), 1983, Antarctica: Glaciological and geophysical folio, Cambridge, U.K., Scott Polar Research Institute, 9 sheets.

Elliot, D. H., 1991. Triassic-Early Cretaceous evolution of Antarctica, in M. R. A. Thomson, A. Crame and J. W. Thomson, ed., Geological evolution of Antarctica: Cambridge, Cambridge University Press, p. 541-548.

Grootes, P.M. and Steig, E.J., 1992, Taylor Dome ice-core study, Antarctic J. U.S., 27(5), p. 57-59.

McElroy, C.T., and Rose, G., Geology of Beacon Heights, Southern Victoria Land, Antarctica, 1:50,000, New Zealand Geological Survey Miscellaneous Series map 15 (1 sheet) and notes. Wellington, DSIR.

Melhuish, A., Bannister, S., ten Brink, U., Beaudoin, B., Stern, T., 1993. Seismic experiment Ross Ice Shelf 1990/91: characteristics of the seismic reflection data. Institute of Geological and Nuclear Sciences Science Report 93/6, 45p.

Morse, D., and Waddington, E., 1992, Glacier geophysical studies for an ice-core site at Taylor Dome: Year two, Antarctic J. U.S., 27(5), p. 59-62.

Stern, T. A. and U.S. ten Brink, 1989. Flexural uplift of the Transantarctic Mountains: $J$. Geophys. Res., 94, p. 10,315-10,330.

ten Brink, U.S., Bannister, S., Beaudoin, B.C., and Stern, T.A., 1993, Geophysical investigations of the tectonic boundary between East and West Antarctica, Science, $261,45-50$.

Webb, P. N., 1991. A review of the Cenozoic stratigraphy and paleontology of Antarctica, in M. R. A. Thomson, J. A. Crame and J. W. Thomson, ed., Gelogoical Evolution of Antarctica: Cambridge, Cambridge University Press, p. 599-607. 


\section{APPENDIX 1: Locations of Surveyed Positions, Shot Numbers, and Magnetic Readings}

by John West and Rebecca Drury

\begin{tabular}{|c|c|c|c|c|c|c|}
\hline Distance (m) & $\begin{array}{c}\text { Flag Loc. } \\
\left.\text { Latitude ( }{ }^{\circ} \mathrm{S}\right)\end{array}$ & $\begin{array}{c}\text { Flag Loc. } \\
\text { Longitude }\left({ }^{\circ} \mathrm{E}\right)\end{array}$ & Elevation (m) & $\begin{array}{c}\text { Shot } \\
\text { Number }\end{array}$ & $\begin{array}{l}\text { Julian } \\
\text { Day }\end{array}$ & $\begin{array}{l}\text { Total Mag. } \\
\text { Field (nT) }\end{array}$ \\
\hline Alpha & 77.7784 & 158.3419 & 2395.60 & & & \\
\hline & $\begin{array}{l}77.7838 \\
77.7811\end{array}$ & $\begin{array}{l}158.3116 \\
158.3054\end{array}$ & 2394.78 & & & $\begin{array}{r}0.0 \\
63555.8\end{array}$ \\
\hline 150 & & $\begin{array}{l}158.3054 \\
158.2992\end{array}$ & 2394.64 & $\begin{array}{l}100 \\
101\end{array}$ & $\begin{array}{l}345 \\
345\end{array}$ & $\begin{array}{l}63555.8 \\
63562.5\end{array}$ \\
\hline $\begin{array}{l}300 \\
450\end{array}$ & $\begin{array}{l}77.7844 \\
777846\end{array}$ & $\begin{array}{l}158.2992 \\
158.2930\end{array}$ & $\begin{array}{l}2394.38 \\
239415\end{array}$ & 102 & $\begin{array}{l}340 \\
345\end{array}$ & $\begin{array}{l}63562.5 \\
63570.6\end{array}$ \\
\hline $\begin{array}{l}450 \\
600\end{array}$ & .77 .7849 & 1582868 & 2394.15 & 103 & 345 & 63580.0 \\
\hline 750 & 777852 & 158.2805 & 2393.99 & 104 & 346 & 635753 \\
\hline 900 & 77.7855 & 158.2743 & 2393.86 & 105 & 346 & 63571.5 \\
\hline 1050 & 77.7857 & 158.2681 & 2393.74 & 106 & 346 & 63563.9 \\
\hline 1200 & 77.7860 & 158.2619 & 2393.74 & 107 & 346 & 63560.0 \\
\hline 1350 & 77.7863 & 158.2557 & 2393.64 & 108 & 346 & 63556.2 \\
\hline 1500 & 77.7865 & 158.2495 & 2393.69 & 109 & 346 & 63539.2 \\
\hline 1650 & 77.7868 & 158.2432 & 2393.68 & 110 & 346 & 63521.3 \\
\hline 1800 & 77.7871 & 158.2370 & 2393.72 & 111 & 346 & 63512.0 \\
\hline 1950 & 77.7873 & 158.2308 & 2393.66 & 112 & 346 & 63511.0 \\
\hline 2100 & 77.7876 & 158.2245 & 2393.39 & .113 & 346 & 63515.5 \\
\hline 2250 & 77.7879 & 158.2183 & 2393.17 & 114 & 346 & 63518.9 \\
\hline 2400 & 77.7882 & 158.2121 & 2392.69 & 115 & 346 & 63523.4 \\
\hline 2550 & 77.7884 & 158.2059 & 2391.93 & 116 & 346 & 63544.6 \\
\hline 2700 & 77.7887 & 158.1997 & 2391.21 & 117 & 346 & 63556.5 \\
\hline 2850 & 77.7890 & 158.1935 & 2390.46 & 118 & 346 & 63567.1 \\
\hline 3000 & 77.7892 & 158.1872 & 2389.71 & 119 & 346 & 63585.9 \\
\hline 3150 & 77.7895 & 158.1810 & 2388.77 & 120 & 346 & 63592.6 \\
\hline 3300 & 77.7898 & 158.1748 & 2387.73 & 121 & 346 & 63613.9 \\
\hline 3450 & 77.7900 & 158.1686 & 2385.92 & 122 & 346 & 63646.3 \\
\hline 3600 & 77.7903 & 158.1623 & 2384.37 & 123 & 346 & 63681.0 \\
\hline 3750 & 77.7906 & 158.1561 & 2382.89 & 124 & 346 & 63706.5 \\
\hline 3900 & 77.7909 & 158.1499 & 2381.43 & 125 & 346 & 63731.2 \\
\hline 4050 & 77.7911 & 158.1437 & 2379.99 & 126 & 346 & 63750.1 \\
\hline 4200 & 77.7914 & 158.1375 & 2378.46 & 127 & 346 & 63753.0 \\
\hline 4350 & 77.7917 & 158.1312 & 2376.73 & 128 & 346 & 63755.4 \\
\hline 4500 & 77.7919 & 158.1250 & 2375.18 & 129 & 346 & 63754.9 \\
\hline 4650 & 77.7922 & 158.1188 & 2373.60 & 130 & 346 & 63745.0 \\
\hline 4800 & 77.7925 & 158.1126 & 2372.34 & 131 & 346 & 63741.4 \\
\hline 4950 & 77.7927 & 158.1064 & 2371.36 & 132 & 346 & 63736.5 \\
\hline 5100 & 77.7930 & 158.1001 & 2370.61 & 133 & 346 & 63737.2 \\
\hline 5250 & 77.7933 & 158.0939 & 2369.89 & 134 & 346 & 63742.2 \\
\hline 5400 & 77.7935 & 158.0877 & 2369.59 & 135 & 346 & 63731.4 \\
\hline 5550 & 77.7938 & 158.0815 & 2369.36 & 136 & 346 & 63733.0 \\
\hline 5700 & 77.7941 & 158.0752 & 2368.96 & 137 & 346 & 63732.9 \\
\hline 5850 & 77.7944 & 158.0690 & 2368.80 & 138 & 346 & 63731.8 \\
\hline 6000 & 77.7946 & 158.0628 & 2368.68 & 139 & 346 & 63734.4 \\
\hline 6150 & 77.7949 & 158.0566 & 2368.40 & 140 & 346 & 63736.7 \\
\hline 6300 & 77.7952 & 158.0504 & 2368.29 & 141 & 346 & 63740.5 \\
\hline 6450 & 7.7 .7955 & 158.0441 & 2368.10 & 142 & 346 & 63742.0 \\
\hline 6600 & 77.7957 & 158.0379 & 2367.87 & 143 & 346 & 63750.8 \\
\hline 6750 & 77.7960 & 158.0317 & 2367.49 & 144 & 346 & 63757.1 \\
\hline 6900 & 77.7963 & 158.0255 & 2366.95 & 145 & 346 & 63770.6 \\
\hline 7050 & 77.7965 & 158.0192 & 2366.62 & 146 & 346 & 63778.0 \\
\hline 7200 & 77.7968 & 158.0130 & 2365.94 & 147 & 346 & 63789.3 \\
\hline 7350 & 77.7971 & 158.0068 & 2365.45 & 148 & 346 & 63793.5 \\
\hline 7500 & 77.7973 & 158.0005 & 2364.70 & 149 & 346 & 63794.0 \\
\hline
\end{tabular}




\begin{tabular}{|c|c|c|c|c|c|c|}
\hline Distance $(m)$ & $\begin{array}{l}\text { Flag Loc. } \\
\left.\text { Latitude (' }{ }^{\circ}\right)\end{array}$ & $\begin{array}{c}\text { Flag Loc. } \\
\left.\text { Longitude ( }{ }^{\circ} \mathrm{E}\right)\end{array}$ & Elevation (m) & $\begin{array}{c}\text { Shot } \\
\text { Number }\end{array}$ & $\begin{array}{l}\text { Julian } \\
\text { Day }\end{array}$ & $\begin{array}{l}\text { Total Mag. } \\
\text { Field (nT) }\end{array}$ \\
\hline 7650 & 77.7976 & 757.9943 & 2363.96 & 750 & 346 & 63793.8 \\
\hline 7800 & 77.7979 & 157.9881 & 2363.33 & 151 & 347 & 63753.1 \\
\hline 8100 & 77.7984 & 157.9756 & 2362.26 & 153 & 347 & 0.0 \\
\hline 8250 & 77.7986 & 157.9694 & 2361.79 & 154 & 347 & 63744.0 \\
\hline 8400 & 77.7989 & 157.9632 & 2361.34 & 155 & 347 & 0.0 \\
\hline 8550 & 77.7992 & 157.9569 & 2360.87 & 156 & 347 & 0.0 \\
\hline 8700 & 77.7994 & 157.9507 & 2360.39 & 157 & 347 & 63726.9 \\
\hline 8850 & 77.7997 & 157.9444 & 2359.96 & 158 & 347 & 0.0 \\
\hline 9000 & 77.7999 & 157.9382 & 2359.30 & 159 & 347 & 0.0 \\
\hline 9150 & 77.8002 & 157.9320 & 2358.79 & 160 & 347 & 63706.9 \\
\hline 9300 & 77.8005 & 157.9257 & 2358.05 & 161 & 347 & 0.0 \\
\hline 9450 & 77.8007 & 157.9195 & 2357.30 & 162 & 347 & 0.0 \\
\hline 9600 & 77.8010 & 157.9132 & 2355.96 & 163 & 347 & 63713.5 \\
\hline 9750 & 77.8012 & 157.9070 & 2354.78 & 164 & 347 & 0.0 \\
\hline 9900 & 77.8015 & 157.9008 & 2353.37 & 165 & 347 & 0.0 \\
\hline 10050 & 77.8018 & 157.8945 & 2351.76 & 166 & 347 & 63680.7 \\
\hline 10200 & 77.8020 & 157.8883 & 2349.69 & 167 & 347 & 0.0 \\
\hline 10350 & 77.8023 & 157.8821 & 2347.30 & 168 & 347 & 0.0 \\
\hline 10500 & 77.8026 & 157.8758 & 2344.39 & 169 & 347 & 63645.3 \\
\hline 10650 & 77.8028 & 157.8696 & 2341.89 & 170 & 347 & 0.0 \\
\hline 10800 & 77.8031 & 157.8634 & 2339.63 & 171 & 347 & 0.0 \\
\hline 10950 & 77.8033 & 157.8571 & 2337.77 & 172 & 347 & 63621.8 \\
\hline 11100 & 77.8036 & 157.8509 & 2336.25 & 173 & 347 & 0.0 \\
\hline 11250 & 77.8039 & 157.8447 & 2335.17 & 174 & 347 & 0.0 \\
\hline 11400 & 77.8041 & 157.8384 & 2334.27 & 175 & 347 & 63579.1 \\
\hline 11550 & 77.8044 & 157.8322 & 2333.74 & 176 & 347 & 0.0 \\
\hline 11700 & 77.8046 & 157.8259 & 2333.22 & 177 & 347 & 0.0 \\
\hline 11850 & 77.8049 & 157.8197 & 2332.89 & 178 & 347 & 63557.2 \\
\hline 12000 & 77.8052 & 157.8135 & 2332.65 & 179 & 347 & 0.0 \\
\hline 12150 & 77.8054 & 157.8072 & 2332.67 & 180 & 347 & 0.0 \\
\hline 12300 & 77.8057 & 157.8010 & 2332.56 & 181 & 347 & 63550.9 \\
\hline 12450 & 77.8059 & 157.7948 & 2332.50 & 182 & 347 & 0.0 \\
\hline 12600 & 77.8062 & 157.7885 & 2332.40 & 183 & 347 & 63550.2 \\
\hline 12750 & 77.8065 & 157.7823 & 2332.32 & 184 & 347 & 63554.2 \\
\hline 12900 & 77.8067 & 157.7760 & 2332.40 & 185 & 347 & 0.0 \\
\hline 13050 & 77.8070 & 157.7698 & 2332.30 & 186 & 347 & 0.0 \\
\hline 13200 & 77.8073 & 157.7636 & 2332.29 & 187 & 347 & 63550.3 \\
\hline 13350 & 77.8075 & 157.7573 & 2332.29 & 188 & 347 & 0.0 \\
\hline 13500 & 77.8078 & 157.7511 & 2332.30 & 189 & 347 & 0.0 \\
\hline 13650 & 77.8080 & 157.7448 & 2332.25 & 190 & 347 & 63548.4 \\
\hline 13800 & 77.8083 & 157.7386 & 2332.30 & 191 & 347 & 0.0 \\
\hline 13950 & 77.8086 & 157.7324 & 2332.19 & 192 & 347 & 0.0 \\
\hline 14100 & 77.8088 & 157.7261 & 2332.40 & 193 & 347 & 63551.7 \\
\hline 14250 & 77.8091 & 157.7199 & 2332.43 & 194 & 347 & 0.0 \\
\hline 14400 & 77.8093 & 157.7137 & 2332.56 & 195 & 347 & 0.0 \\
\hline 14550 & 77.8096 & 157.7074 & 2332.71 & 196 & 347 & 63550.5 \\
\hline 14700 & 77.8099 & 157.7012 & 2332.96 & 197 & 347 & 63549.4 \\
\hline 14850 & 77.8101 & 157.6949 & 2333.17 & 198 & 347 & 0.0 \\
\hline 15000 & 77.8104 & 157.6887 & 2333.47 & 199 & 347 & 63557.4 \\
\hline 15150 & 77.8106 & 157.6824 & 2333.55 & 200 & 347 & 0.0 \\
\hline 15300 & 77.8109 & 157.6762 & 2333.71 & 201 & 347 & 0.0 \\
\hline 15450 & 77.8112 & 157.6700 & 2333.88 & 202 & 347 & 63579.0 \\
\hline
\end{tabular}




\begin{tabular}{|c|c|c|c|c|c|c|}
\hline Distance (m) & $\begin{array}{l}\text { Flag Loc. } \\
\text { Latitude ('s) }\end{array}$ & $\begin{array}{c}\text { Flag Loc. } \\
\left.\text { Longitude ( }{ }^{\circ} \mathrm{E}\right)\end{array}$ & Elevation (m) & $\begin{array}{l}\text { Shot } \\
\text { Number }\end{array}$ & $\begin{array}{c}\text { Julian } \\
\text { Day }\end{array}$ & $\begin{array}{l}\text { Total Mag. } \\
\text { Field (nT) }\end{array}$ \\
\hline 15600 & 77.8114 & $\overline{157.6637}$ & 2333.87 & $\overline{203}$ & 347 & 0.0 \\
\hline 15750 & 77.8117 & 157.6575 & 2333.82 & 204 & 347 & 63591.8 \\
\hline 15900 & 77.8120 & 157.6512 & 2333.78 & 205 & 347 & 63608.2 \\
\hline 16050 & 77.8122 & 157.6450 & 2333.44 & 206 & 347 & 0.0 \\
\hline 16200 & 77.8125 & 157.6388 & 2333.11 & 207 & 347 & 0.0 \\
\hline 16350 & 77.8127 & 157.6325 & 2332.61 & 208 & 347 & 63659.5 \\
\hline 16500 & 77.8130 & 157.6263 & 2332.12 & 209 & 347 & 0.0 \\
\hline 16650 & 77.8133 & 157.6200 & 2331.48 & 210 & 347 & 0.0 \\
\hline 16800 & 77.8135 & 157.6138 & 2330.72 & 211 & 347 & 63704.6 \\
\hline 16950 & 77.8138 & 157.6076 & 2329.82 & 212 & 347 & 0.0 \\
\hline 17100 & 77.8140 & 157.6013 & 2329.00 & 213 & 347 & 0.0 \\
\hline 17250 & 77.8143 & 157.5951 & 2328.10 & 214 & 347 & 63733.5 \\
\hline 17400 & 77.8146 & 157.5889 & 2327.21 & 215 & 347 & 0.0 \\
\hline 17550 & 77.8148 & 157.5826 & 2326.56 & 216 & 347 & 0.0 \\
\hline 17700 & 77.8151 & 157.5764 & 2325.75 & 217 & 347 & 63759.3 \\
\hline 17850 & 77.8153 & 157.5701 & 2324.83 & 218 & 347 & 0.0 \\
\hline 18000 & 77.8156 & 157.5639 & 2324.38 & 219 & 347 & 63775.1 \\
\hline 18150 & 77.8159 & 157.5576 & 2323.62 & 220 & 348 & 63748.9 \\
\hline 18300 & 77.8161 & 157.5514 & 2322.94 & 221 & 348 & 0.0 \\
\hline 18450 & 77.8164 & 157.5451 & 2321.98 & 222 & 348 & 0.0 \\
\hline 18600 & 77.8167 & 157.5389 & 2321.33 & 223 & 348 & 63793.8 \\
\hline 18750 & 77.8169 & 157.5327 & 2320.70 & 224 & 348 & 0.0 \\
\hline 18900 & 77.8172 & 157.5264 & 2320.29 & 225 & 348 & 0.0 \\
\hline 19050 & 77.8175 & 157.5202 & 2319.78 & 226 & 348 & 63787.9 \\
\hline 19200 & 77.8177 & 157.5139 & 2319.53 & 227 & 348 & 0.0 \\
\hline 19350 & 77.8180 & 157.5077 & 2319.35 & 228 & 348 & 0.0 \\
\hline 19500 & 77.8182 & 157.5014 & 2319.35 & 229 & 348 & 63777.8 \\
\hline 19650 & 77.8185 & 157.4952 & 2319.31 & 230 & 348 & 0.0 \\
\hline 19800 & 77.8188 & 157.4890 & 2319.40 & 231 & 348 & 0.0 \\
\hline 19950 & 77.8190 & 157.4827 & 2319.35 & 232 & 348 & 63739.7 \\
\hline 20100 & 77.8193 & 157.4765 & 2319.24 & 233 & 348 & 63731.8 \\
\hline 20250 & 77.8196 & 157.4702 & 2318.83 & 234 & 348 & 0.0 \\
\hline 20400 & 77.8198 & 157.4640 & 2318.30 & 235 & 348 & 63718.8 \\
\hline 20550 & 77.8201 & 157.4577 & 2317.36 & 236 & 348 & 0.0 \\
\hline 20700 & 77.8204 & 157.4515 & 2316.17 & 237 & 348 & 0.0 \\
\hline 20850 & 77.8206 & 157.4453 & 2314.90 & 238 & 348 & 63739.6 \\
\hline 21000 & 77.8209 & 157.4390 & 2313.41 & 239 & 348 & 0.0 \\
\hline 21150 & 77.8211 & 157.4328 & 2312.45 & 240 & 348 & 0.0 \\
\hline 21300 & 77.8214 & 157.4265 & 2311.49 & 241 & 348 & 63754.0 \\
\hline 21450 & 77.8217 & 157.4203 & 2310.70 & 242 & 348 & 0.0 \\
\hline 21600 & 77.8219 & 157.4140 & 2310.24 & 243 & 348 & 0.0 \\
\hline 21750 & 77.8222 & 157.4078 & 2309.80 & 244 & 348 & 63758.2 \\
\hline 21900 & 77.8224 & 157.4016 & 2309.65 & 245 & 348 & 0.0 \\
\hline 22050 & 77.8227 & 157.3953 & 2309.37 & 246 & 348 & 0.0 \\
\hline 22200 & 77.8230 & 157.3890 & 2309.20 & 247 & 348 & 63735.4 \\
\hline 22350 & 77.8232 & 157.3828 & 2308.80 & 248 & 348 & 0.0 \\
\hline 22500 & 77.8235 & 157.3765 & 2308.60 & 249 & 348 & 0.0 \\
\hline 22650 & 77.8237 & 157.3703 & 2308.43 & 250 & 348 & 63705.1 \\
\hline 22800 & 77.8240 & 157.3640 & 2308.38 & 251 & 348 & 0.0 \\
\hline 22950 & 77.8242 & 157.3578 & 2308.33 & 252 & 348 & 0.0 \\
\hline 23100 & 77.8245 & 157.3515 & 2308.36 & 253 & 348 & 63654.2 \\
\hline 23250 & 77.8247 & 157.3452 & 2308.45 & 254 & 348 & 0.0 \\
\hline
\end{tabular}




\begin{tabular}{|c|c|c|c|c|c|c|}
\hline Distance (m) & $\begin{array}{l}\text { Flag Loc. } \\
\text { Latitude ('S) }\end{array}$ & $\begin{array}{c}\text { Flag Loc. } \\
\text { Longitude }\left({ }^{\circ} \mathrm{E}\right)\end{array}$ & Elevation (m) & $\begin{array}{c}\text { Shot } \\
\text { Number }\end{array}$ & $\begin{array}{c}\text { Julian } \\
\text { Day }\end{array}$ & $\begin{array}{l}\text { Total Mag. } \\
\text { Field (nT) }\end{array}$ \\
\hline 23400 & 77.8250 & 157.3390 & 2308.59 & 255 & 348 & 0.0 \\
\hline 23550 & 77.8252 & 157.3327 & 2308.45 & 256 & 349 & 63646.5 \\
\hline 23700 & 77.8255 & 157.3265 & 2308.44 & 257 & 349 & 0.0 \\
\hline 23850 & 77.8257 & 157.3202 & 2308.14 & 258 & 349 & 0.0 \\
\hline 24000 & 77.8260 & 157.3139 & 2307.47 & 259 & 349 & 63635.5 \\
\hline 24150 & 77.8262 & 157.3077 & 2306.49 & 260 & 349 & 0.0 \\
\hline 24300 & 77.8265 & 157.3014 & 2305.35 & 261 & 349 & 0.0 \\
\hline 24450 & 77.8267 & 157.2952 & 2304.02 & 262 & 349 & 63639.9 \\
\hline 24600 & 77.8270 & 157.2889 & 2302.59 & 263 & 349 & 0.0 \\
\hline 24750 & 77.8272 & 157.2827 & 2301.02 & 264 & 349 & 0.0 \\
\hline 24900 & 77.8275 & 157.2764 & 2299.63 & 265 & 349 & 63686.4 \\
\hline 25050 & 77.8277 & 157.2701 & 2298.55 & 266 & 349 & 0.0 \\
\hline 25200 & 77.8280 & 157.2639 & 2297.69 & 267 & 349 & 0.0 \\
\hline 25350 & 77.8282 & 157.2576 & 2297.02 & 268 & 349 & 63744.8 \\
\hline 25500 & 77.8285 & 157.2514 & 2296.44 & 269 & 349 & 0.0 \\
\hline 25650 & 77.8287 & 157.2451 & 2296.03 & 270 & 349 & 0.0 \\
\hline 25800 & 77.8289 & 157.2389 & 2295.55 & 271 & 349 & 63780.4 \\
\hline 25950 & 77.8292 & 157.2326 & 2294.74 & 272 & 349 & 0.0 \\
\hline 26100 & 77.8295 & 157.2263 & 2293.94 & 273 & 349 & 63803.7 \\
\hline 26250 & 77.8297 & 157.2200 & 2292.78 & 274 & 349 & 63824.3 \\
\hline 26400 & 77.8300 & 157.2138 & 2291.21 & 275 & 349 & 0.0 \\
\hline 26550 & 77.8302 & 157.2075 & 2289.43 & 276 & 349 & 0.0 \\
\hline 26700 & 77.8305 & 157.2013 & 2287.60 & 277 & 351 & 63742.2 \\
\hline 26850 & 77.8307 & 157.1950 & 2285.63 & 278 & 351 & 0.0 \\
\hline 27000 & 77.8310 & 157.1888 & 2284.11 & 279 & 351 & 0.0 \\
\hline 27150 & 77.8312 & 157.1825 & 2282.89 & 280 & 351 & 63761.8 \\
\hline 27300 & 77.8315 & 157.1762 & 2282.08 & 281 & 351 & 0.0 \\
\hline 27450 & 77.8317 & 157.1700 & 2281.41 & 282 & 351 & 0.0 \\
\hline 27600 & 77.8319 & 157.1637 & 2280.99 & 283 & 351 & 63716.2 \\
\hline 27750 & 77.8322 & 157.1575 & 2280.33 & 284 & 351 & 0.0 \\
\hline 27900 & 77.8324 & 157.1512 & 2279.89 & 285 & 351 & 0.0 \\
\hline 28050 & 77.8327 & 157.1449 & 2279.31 & 286 & 351 & 63714.0 \\
\hline 28200 & 77.8330 & 157.1387 & 2278.61 & 287 & 351 & 63657.1 \\
\hline 28350 & 77.8332 & 157.1324 & 2277.88 & 288 & 351 & 0.0 \\
\hline 28500 & 77.8335 & 157.1261 & 2277.20 & 289 & 351 & 63582.4 \\
\hline 28650 & 77.8337 & 157.1199 & 2276.46 & 290 & 351 & 0.0 \\
\hline 28800 & 77.8339 & 157.1136 & 2275.63 & 291 & 351 & 0.0 \\
\hline 28950 & 77.8342 & 157.1073 & 2274.65 & 292 & 351 & 63550.7 \\
\hline 29100 & 77.8344 & 157.1011 & 2273.58 & 293 & 351 & 0.0 \\
\hline 29250 & 77.8347 & 157.0948 & 2272.34 & 294 & 351 & 0.0 \\
\hline 29400 & 77.8349 & 157.0886 & 2270.92 & 295 & 351 & 63528.6 \\
\hline 29550 & 77.8352 & 157.0823 & 2269.49 & 296 & 351 & 0.0 \\
\hline 29700 & 77.8354 & 157.0760 & 2268.27 & 297 & 351 & 63512.4 \\
\hline 29850 & 77.8357 & 157.0698 & 2266.86 & 298 & 351 & 63522.0 \\
\hline 30000 & 77.8359 & 157.0635 & 2265.57 & 299 & 351 & 0.0 \\
\hline 30150 & 77.8362 & 157.0572 & 2264.28 & 300 & 351 & 0.0 \\
\hline 30300 & 77.8364 & 157.0510 & 2263.06 & 301 & 351 & 63508.9 \\
\hline 30450 & 77.8367 & 157.0447 & 2261.95 & 302 & 351 & 0.0 \\
\hline 30600 & 77.8369 & 157.0385 & 2260.83 & 303 & 351 & 0.0 \\
\hline 30750 & 77.8372 & 157.0322 & 2259.75 & 304 & 351 & 63534.7 \\
\hline 30900 & 77.8374 & 157.0259 & 2258.86 & 305 & 351 & 0.0 \\
\hline 31050 & 77.8377 & 157.0197 & 2257.87 & 306 & 351 & 0.0 \\
\hline
\end{tabular}




\begin{tabular}{|c|c|c|c|c|c|c|}
\hline Distance (m) & $\begin{array}{l}\text { Flag Loc. } \\
\text { Latitude ('s) }\end{array}$ & $\begin{array}{c}\text { Flag Loc. } \\
\text { Longitude }\left({ }^{\circ} \mathrm{E}\right)\end{array}$ & Elevation (m) & $\begin{array}{c}\text { Shot } \\
\text { Number }\end{array}$ & $\begin{array}{c}\text { Julian } \\
\text { Day }\end{array}$ & $\begin{array}{l}\text { Total Mag. } \\
\text { Field (nT) }\end{array}$ \\
\hline 31200 & 77.8379 & 157.0134 & 2257.06 & 307 & 351 & 63540.4 \\
\hline 31350 & 77.8382 & 157.0071 & 2256.05 & 308 & 351 & 0.0 \\
\hline 31500 & 77.8384 & 157.0009 & 2255.35 & 309 & 351 & 0.0 \\
\hline 31650 & 77.8387 & 156.9946 & 2254.54 & 310 & 351 & 63548.4 \\
\hline 31800 & 77.8389 & 156.9883 & 2253.78 & 311 & 351 & 63555.7 \\
\hline 31950 & 77.8392 & 156.9820 & 2252.89 & 312 & 351 & 0.0 \\
\hline 32100 & 77.8394 & 156.9758 & 2252.11 & 313 & 351 & 63559.1 \\
\hline 32250 & 77.8397 & 156.9695 & 2251.28 & 314 & 351 & 0.0 \\
\hline 32400 & 77.8399 & 156.9633 & 2250.37 & 315 & 351 & 0.0 \\
\hline 32550 & 77.8402 & 156.9570 & 2249.50 & 316 & 351 & 63567.2 \\
\hline 32700 & 77.8404 & 156.9507 & 2248.72 & 317 & 351 & 0.0 \\
\hline 32850 & 77.8407 & 156.9445 & 2247.87 & 318 & 351 & 0.0 \\
\hline 33000 & 77.8409 & 156.9382 & 2247.13 & 319 & 351 & 63566.5 \\
\hline 33150 & 77.8412 & 156.9319 & 2246.30 & 320 & 351 & 0.0 \\
\hline 33300 & 77.8414 & 156.9257 & 2245.52 & 321 & 351 & 0.0 \\
\hline 33450 & 77.8417 & 156.9194 & 2244.51 & 322 & 351 & 63592.5 \\
\hline 33600 & 77.8419 & 156.9131 & 2243.30 & 323 & 351 & 0.0 \\
\hline 33750 & 77.8422 & 156.9069 & 2242.12 & 324 & 351 & 0.0 \\
\hline 33900 & 77.8424 & 156.9006 & 2241.04 & 325 & 351 & 63624.8 \\
\hline 34050 & 77.8427 & 156.8943 & 2239.89 & 326 & 351 & 0.0 \\
\hline 34200 & 77.8429 & 156.8880 & 2238.59 & 327 & 351 & 0.0 \\
\hline 34350 & 77.8432 & 156.8818 & 2237.24 & 328 & 351 & 63623.2 \\
\hline 34500 & 77.8434 & 156.8755 & 2235.79 & 329 & 351 & 0.0 \\
\hline 34650 & 77.8437 & 156.8692 & 2233.90 & 330 & 351 & 0.0 \\
\hline 34800 & 77.8439 & 156.8630 & 2231.40 & 331 & 351 & 63603.1 \\
\hline 34950 & 77.8442 & 156.8567 & 2228.50 & 332 & 351 & 0.0 \\
\hline 35100 & 77.8444 & 156.8504 & 2225.12 & 333 & 351 & 0.0 \\
\hline 35250 & 77.8447 & 156.8442 & 2221.12 & 334 & 351 & 63574.0 \\
\hline 35400 & 77.8449 & 156.8379 & 2216.93 & 335 & 351 & 0.0 \\
\hline 35550 & 77.8452 & 156.8316 & 2212.74 & 336 & 351 & 0.0 \\
\hline 35700 & 77.8454 & 156.8254 & 2208.75 & 337 & 351 & 63571.7 \\
\hline 35850 & 77.8457 & 156.8191 & 2205.04 & 338 & 351 & 0.0 \\
\hline 36000 & 77.8459 & 156.8129 & 2202.04 & 339 & 351 & 0.0 \\
\hline 36150 & 77.8462 & 156.8066 & 2199.56 & 340 & 351 & 63577.5 \\
\hline 36300 & 77.8464 & 156.8003 & 2197.49 & 341 & 351 & 0.0 \\
\hline 36450 & 77.8467 & 156.7941 & 2195.81 & 342 & 351 & 0.0 \\
\hline 36600 & 77.8469 & 156.7878 & 2194.37 & 343 & 351 & 63566.7 \\
\hline 36750 & 77.8472 & 156.7815 & 2193.36 & 344 & 351 & 0.0 \\
\hline 36900 & 77.8474 & 156.7752 & 2192.76 & 345 & 351 & 63559.7 \\
\hline 37050 & 77.8477 & 156.7690 & 2192.09 & 346 & 351 & 63562.7 \\
\hline 37200 & 77.8479 & 156.7627 & 2191.63 & 347 & 351 & 0.0 \\
\hline 37350 & 77.8482 & 156.7564 & 2191.35 & 348 & 351 & 0.0 \\
\hline 37500 & 77.8484 & 156.7502 & 2191.07 & 349 & 353 & 63543.7 \\
\hline 37650 & 77.8487 & 156.7439 & 2190.83 & 350 & 353 & 0.0 \\
\hline 37800 & 77.8489 & 156.7376 & 2190.65 & 351 & 353 & 0.0 \\
\hline 37950 & 77.8492 & 156.7314 & 2190.60 & 352 & 353 & 0.0 \\
\hline 38100 & 77.8494 & 156.7251 & 2190.60 & 353 & 353 & 0.0 \\
\hline 38250 & 77.8497 & 156.7188 & 2190.67 & 354 & 353 & 0.0 \\
\hline 38400 & 77.8499 & 156.7125 & 2190.78 & 355 & 353 & 0.0 \\
\hline 38550 & 77.8502 & 156.7063 & 2190.84 & 356 & 353 & 0.0 \\
\hline 38700 & 77.8504 & 156.7000 & 2190.91 & 357 & 353 & 0.0 \\
\hline 38850 & 77.8507 & 156.6937 & 2191.06 & 358 & 353 & 0.0 \\
\hline
\end{tabular}




\begin{tabular}{|c|c|c|c|c|c|c|}
\hline Distance (m) & $\begin{array}{l}\text { Flag Loc. } \\
\text { Latitude (' } \mathbf{S})\end{array}$ & $\begin{array}{c}\text { Flag Loc. } \\
\text { Longitude }\left({ }^{\circ} E\right)\end{array}$ & Elevation (m) & $\begin{array}{c}\text { Shot } \\
\text { Number }\end{array}$ & $\begin{array}{c}\text { Julian } \\
\text { Day }\end{array}$ & $\begin{array}{l}\text { Total Mag. } \\
\text { Field (nT) }\end{array}$ \\
\hline 39000 & 77.8509 & $\overline{756.6874}$ & 2797.36 & $\overline{359}$ & $\overline{353}$ & $\overline{0.0}$ \\
\hline 39150 & 77.8512 & 156.6812 & 2191.52 & 360 & 353 & 0.0 \\
\hline 39300 & 77.8514 & 156.6749 & 2191.78 & 361 & 353 & 0.0 \\
\hline 39450 & 77.8516 & 156.6686 & 2191.97 & 362 & 353 & 0.0 \\
\hline 39600 & 77.8519 & 156.6623 & 2192.45 & 363 & 353 & 0.0 \\
\hline 39750 & 77.8521 & 156.6560 & 2192.76 & 364 & 353 & 0.0 \\
\hline 39900 & 77.8523 & 156.6497 & 2193.16 & 365 & 353 & 0.0 \\
\hline 40050 & 77.8526 & 156.6434 & 2193.47 & 366 & 353 & 0.0 \\
\hline 40200 & 77.8528 & 156.6372 & 2193.94 & 367 & 353 & 0.0 \\
\hline 40350 & 77.8530 & 156.6309 & 2194.21 & 368 & 353 & 0.0 \\
\hline 40500 & 77.8533 & 156.6246 & 2194.75 & 369 & 353 & 0.0 \\
\hline 40650 & 77.8535 & 156.6183 & 2195.05 & 370 & 353 & 0.0 \\
\hline 40800 & 77.8537 & 156.6120 & 2195.45 & 371 & 353 & 0.0 \\
\hline 40950 & 77.8540 & 156.6057 & 2195.92 & 372 & 353 & 0.0 \\
\hline 41100 & 77.8542 & 156.5994 & 2196.16 & 373 & 353 & 0.0 \\
\hline 41250 & 77.8544 & 156.5931 & 2196.67 & 374 & 353 & 0.0 \\
\hline 41400 & 77.8547 & 156.5869 & 2197.33 & 375 & 353 & 0.0 \\
\hline 41550 & 77.8549 & 156.5806 & 2197.91 & 376 & 353 & 0.0 \\
\hline 41700 & 77.8551 & 156.5743 & 2198.67 & 377 & 353 & 0.0 \\
\hline 41850 & 77.8554 & 156.5680 & 2199.36 & 378 & 353 & 0.0 \\
\hline 42000 & 77.8556 & 156.5617 & 2200.20 & 379 & 353 & 0.0 \\
\hline 42150 & 77.8559 & 156.5554 & 2201.02 & 380 & 353 & 0.0 \\
\hline 42300 & 77.8561 & 156.5491 & 2201.85 & 381 & 353 & 0.0 \\
\hline 42450 & 77.8563 & 156.5429 & 2202.64 & 382 & 353 & 0.0 \\
\hline 42600 & 77.8566 & 156.5366 & 2203.49 & 383 & 353 & 0.0 \\
\hline 42750 & 77.8568 & 156.5303 & 2204.46 & 384 & 353 & 0.0 \\
\hline 42900 & 77.8570 & 156.5240 & 2205.43 & 385 & 353 & 0.0 \\
\hline 43050 & 77.8573 & 156.5177 & 2206.28 & 386 & 353 & 0.0 \\
\hline 43200 & 77.8575 & 156.5114 & 2207.15 & 387 & 353 & 0.0 \\
\hline 43350 & 77.8577 & 156.5051 & 2207.80 & 388 & 353 & 0.0 \\
\hline 43500 & 77.8580 & 156.4989 & 2208.67 & 389 & 353 & 0.0 \\
\hline 43650 & 77.8582 & 156.4926 & 2209.61 & 390 & 353 & 0.0 \\
\hline 43800 & 77.8585 & 156.4863 & 2210.35 & 391 & 353 & 0.0 \\
\hline 43950 & 77.8587 & 156.4800 & 2211.00 & 392 & 353 & 0.0 \\
\hline 44100 & 77.8589 & 156.4737 & 2211.77 & 393 & 353 & 0.0 \\
\hline 44250 & 77.8592 & 156.4674 & 2212.23 & 394 & 353 & 0.0 \\
\hline 44400 & 77.8594 & 156.4612 & 2212.75 & 395 & 353 & 0.0 \\
\hline 44550 & 77.8596 & 156.4549 & 2213.43 & 396 & 353 & 0.0 \\
\hline 44700 & 77.8599 & 156.4486 & 2213.87 & 397 & 353 & 0.0 \\
\hline 44850 & 77.8601 & 156.4423 & 2214.30 & 398 & 353 & 0.0 \\
\hline 45000 & 77.8604 & 156.4360 & 2214.68 & 399 & 353 & 0.0 \\
\hline 45150 & 77.8606 & 156.4297 & 2215.03 & 400 & 353 & 0.0 \\
\hline 45300 & 77.8608 & 156.4234 & 2215.35 & 401 & 353 & 0.0 \\
\hline 45450 & 77.8611 & 156.4171 & 2215.85 & 402 & 353 & 0.0 \\
\hline 45600 & 77.8613 & 156.4109 & 2216.15 & 403 & 353 & 0.0 \\
\hline 45750 & 77.8615 & 156.4046 & 2216.36 & 404 & 353 & 0.0 \\
\hline 45900 & 77.8618 & 156.3983 & 2216.70 & 405 & 353 & 0.0 \\
\hline 46050 & 77.8620 & 156.3920 & 2216.94 & 406 & 353 & 0.0 \\
\hline 46200 & 77.8622 & 156.3857 & 2217.25 & 407 & 353 & 0.0 \\
\hline 46350 & 77.8625 & 156.3794 & 2217.51 & 408 & 353 & 0.0 \\
\hline 46500 & 77.8627 & 156.3731 & 2217.82 & 409 & 353 & 0.0 \\
\hline 46650 & 77.8630 & 156.3668 & 2218.10 & 410 & 353 & 0.0 \\
\hline
\end{tabular}




\begin{tabular}{|c|c|c|c|c|c|c|}
\hline Distance $(m)$ & $\begin{array}{l}\text { Flag Loc. } \\
\text { Latitude (' } \mathbf{S})\end{array}$ & $\begin{array}{c}\text { Flag Loc. } \\
\left.\text { Longitude ( }{ }^{\circ} \mathrm{E}\right)\end{array}$ & Elevation (m) & $\begin{array}{c}\text { Shot } \\
\text { Number }\end{array}$ & $\begin{array}{c}\text { Julian } \\
\text { Day }\end{array}$ & $\begin{array}{l}\text { Total Mag. } \\
\text { Field (nT) }\end{array}$ \\
\hline 46800 & 77.8632 & 156.3605 & 2218.32 & 411 & 353 & $\overline{0.0}$ \\
\hline 46950 & 77.8634 & 156.3543 & 2218.58 & 412 & 354 & 63535.1 \\
\hline 47100 & 77.8637 & 156.3480 & 2218.76 & 413 & 354 & 0.0 \\
\hline 47250 & 77.8639 & 156.3417 & 2219.20 & 414 & 354 & 0.0 \\
\hline 47400 & 77.8641 & 156.3354 & 2219.53 & 415 & 354 & 63497.3 \\
\hline 47550 & 77.8644 & 156.3291 & 2220.11 & 416 & 354 & 0.0 \\
\hline 47700 & 77.8646 & 156.3228 & 2220.62 & 417 & 354 & 0.0 \\
\hline 47850 & 77.8649 & 156.3165 & 2221.09 & 418 & 354 & 63505.2 \\
\hline 48000 & 77.8651 & 156.3103 & 2221.92 & 419 & 354 & 0.0 \\
\hline 48150 & 77.8653 & 156.3040 & 2222.50 & 420 & 354 & 0.0 \\
\hline 48300 & 77.8656 & 156.2977 & 2223.11 & 421 & 354 & 63491.5 \\
\hline 48450 & 77.8658 & 156.2914 & 2223.73 & 422 & 354 & 0.0 \\
\hline 48600 & 77.8660 & 156.2851 & 2224.37 & 423 & 354 & 0.0 \\
\hline 48750 & 77.8663 & 156.2788 & 2224.88 & 424 & 354 & 63481.2 \\
\hline 48900 & 77.8665 & 156.2725 & 2225.30 & 425 & 354 & 0.0 \\
\hline 49050 & 77.8668 & 156.2662 & 2225.64 & 426 & 354 & 0.0 \\
\hline 49200 & 77.8670 & 156.2599 & 2226.00 & 427| & 354 & 63476.7 \\
\hline 49350 & 77.8672 & 156.2536 & 2226.16 & 428 & 354 & 0.0 \\
\hline 49500 & 77.8675 & 156.2473 & 2226.23 & 429 & 354 & 0.0 \\
\hline 49650 & 77.8677 & 156.2411 & 2226.49 & 430 & 354 & 63477.8 \\
\hline 49800 & 77.8679 & 156.2348 & 2226.43 & 431 & 354 & 0.0 \\
\hline 49950 & 77.8682 & 156.2285 & 2226.58 & 432 & 354 & 0.0 \\
\hline 50100 & 77.8684 & 156.2222 & 2226.40 & 433 & 354 & 63475.0 \\
\hline 50250 & 77.8686 & 156.2159 & 2226.38 & 434 & 354 & 0.0 \\
\hline 50400 & 77.8689 & 156.2096 & 2226.32 & 435 & 354 & 0.0 \\
\hline 50550 & 77.8691 & 156.2033 & 2226.04 & 436 & 354 & 63470.6 \\
\hline 50700 & 77.8693 & 156.1970 & 2226.16 & 437 & 354 & 63474.9 \\
\hline 50850 & 77.8696 & 156.1907 & 2225.96 & 438 & 354 & 0.0 \\
\hline 51000 & 77.8698 & 156.1844 & 2225.81 & 439 & 354 & 63453.2 \\
\hline 51150 & 77.8701 & 156.1781 & 2225.63 & 440 & 354 & 0.0 \\
\hline 51300 & 77.8703 & 156.1718 & 2225.42 & 441 & 354 & 0.0 \\
\hline 51450 & 77.8705 & 156.1655 & 2225.29 & 442 & 354 & 63451.5 \\
\hline 51600 & 77.8708 & 156.1593 & 2224.94 & 443 & 354 & 0.0 \\
\hline 51750 & 77.8710 & 156.1530 & 2224.86 & 444 & 354 & 0.0 \\
\hline 51900 & 77.8712 & 156.1467 & 2224.63 & 445 & 354 & 63486.2 \\
\hline 52050 & 77.8715 & 156.1404 & 2224.27 & 446 & 354 & 0.0 \\
\hline 52200 & 77.8717 & 156.1341 & 2223.96 & 447 & 354 & 0.0 \\
\hline 52350 & 77.8719 & 156.1278 & 2223.70 & 448 & 354 & 63487.5 \\
\hline 52500 & 77.8722 & 156.1215 & 2223.17 & 449 & 354 & 0.0 \\
\hline 52650 & 77.8724 & 156.1152 & 2222.85 & 450 & 354 & 0.0 \\
\hline 52800 & 77.8727 & 156.1089 & 2222.82 & 451 & 354 & 63490.2 \\
\hline 52950 & 77.8729 & 156.1026 & 2222.48 & 452 & 354 & 0.0 \\
\hline 53100 & 77.8731 & 156.0963 & 2221.90 & 453 & 354 & 0.0 \\
\hline 53250 & 77.8734 & 156.0900 & 2221.62 & 454 & 354 & 63512.3 \\
\hline 53400 & 77.8736 & 156.0837 & 2221.15 & 455 & 354 & 0.0 \\
\hline 53550 & 77.8738 & 156.0774 & 2220.69 & 456 & 354 & 0.0 \\
\hline 53700 & 77.8741 & 156.0711 & 2220.11 & 457| & 354 & 63514.0 \\
\hline 53850 & 77.8743 & 156.0648 & 2219.37 & 458 & 354 & 0.0 \\
\hline 54000 & 77.8745 & 156.0585 & 2218.76 & 459 & 354 & 0.0 \\
\hline 54150 & 77.8748 & 156.0522 & 2217.99 & 460 & 354 & 63530.1 \\
\hline 54300 & 77.8750 & 156.0460 & 2216.99 & 461 & 354 & 0.0 \\
\hline 54450 & 77.8752 & 156.0397 & 2216.02 & 462 & 354 & 0.0 \\
\hline
\end{tabular}




\begin{tabular}{|c|c|c|c|c|c|c|}
\hline Distance $(m)$ & $\begin{array}{c}\text { Flag Loc. } \\
\text { Latitude }\left({ }^{\circ} \mathbf{S}\right)\end{array}$ & $\begin{array}{c}\text { Flag Loc. } \\
\left.\text { Longitude ( }{ }^{\circ} \mathrm{E}\right)\end{array}$ & Elevation (m) & $\begin{array}{c}\text { Shot } \\
\text { Number }\end{array}$ & $\begin{array}{l}\text { Julian } \\
\text { Day }\end{array}$ & $\begin{array}{l}\text { Total Mag. } \\
\text { Field (nT) }\end{array}$ \\
\hline 54600 & 77.8755 & 156.0333 & 2215.42 & 463 & 354 & 63551.9 \\
\hline 54750 & 77.8757 & 156.0271 & 2214.36 & 464 & 354 & 0.0 \\
\hline 54900 & 77.8760 & 156.0208 & 2213.27 & 465 & 354 & 0.0 \\
\hline 55050 & 77.8762 & 156.0145 & 2212.07 & 466 & 354 & 63616.5 \\
\hline 55200 & 77.8764 & 156.0082 & 2211.02 & 467 & 354 & 0.0 \\
\hline 55350 & 77.8767 & 156.0019 & 2209.85 & 468 & 354 & 0.0 \\
\hline 55500 & 77.8769 & 155.9956 & 2208.77 & 469 & 354 & 63634.6 \\
\hline 55650 & 77.8771 & 155.9893 & 2207.60 & 470 & 354 & 0.0 \\
\hline 55800 & 77.8774 & 155.9830 & 2206.66 & 471 & 354 & 0.0 \\
\hline 55950 & 77.8776 & 155.9767 & 2205.75 & 472 & 354 & 63649.5 \\
\hline 56100 & 77.8778 & 155.9704 & 2204.77 & 473 & 354 & 0.0 \\
\hline 56250 & 77.8781 & 155.9641 & 2203.89 & 474 & 354 & 0.0 \\
\hline 56400 & 77.8783 & 155.9578 & 2203.31 & 475 & 354 & 63657.0 \\
\hline 56550 & 77.8785 & 155.9515 & 2202.43 & 476 & 354 & 0.0 \\
\hline 56700 & 77.8788 & 155.9452 & 2201.88 & 477 & 354 & 63647.2 \\
\hline 56850 & 77.8790 & 155.9389 & 2201.22 & 478 & 354 & 63653.2 \\
\hline 57000 & 77.8792 & 155.9326 & 2200.70 & 479 & 354 & 0.0 \\
\hline 57150 & 77.8794 & 155.9263 & 2200.25 & 480 & 354 & 0.0 \\
\hline 57300 & 77.8797 & 155.9200 & 2199.70 & 481 & 354 & 63658.7 \\
\hline 57450 & 77.8799 & 155.9136 & 2199.39 & 482 & 354 & 0.0 \\
\hline 57600 & 77.8801 & 155.9073 & 2199.00 & 483 & 354 & 0.0 \\
\hline 57750 & 77.8803 & 155.9010 & 2198.66 & 484 & 354 & 63652.3 \\
\hline 57900 & 77.8805 & 155.8947 & 2198.35 & 485 & 354 & 0.0 \\
\hline 58050 & 77.8807 & 155.8884 & 2198.15 & 486 & 354 & 0.0 \\
\hline 58200 & 77.8810 & 155.8821 & 2198.12 & 487 & 354 & 63650.3 \\
\hline 58350 & 77.8812 & 155.8758 & 2197.86 & 488 & 354 & 0.0 \\
\hline 58500 & 77.8814 & 155.8695 & 2197.86 & 489 & 354 & 0.0 \\
\hline 58650 & 77.8816 & 155.8631 & 2197.72 & 490 & 354 & 63648.4 \\
\hline 58800 & 77.8818 & 155.8568 & 2197.85 & 491 & 354 & 63654.3 \\
\hline 58950 & 77.8821 & 155.8505 & 2197.87 & 492 & 354 & 0.0 \\
\hline 59100 & 77.8823 & 155.8442 & 2197.92 & 493 & 354 & 63651.6 \\
\hline 59250 & 77.8825 & 155.8379 & 2198.12 & 494 & 354 & 0.0 \\
\hline 59400 & 77.8827 & 155.8316 & 2198.36 & 495 & 354 & 0.0 \\
\hline 59550 & 77.8829 & 155.8252 & 2198.45 & 496 & 355 & 63651.0 \\
\hline 59700 & 77.8831 & 155.8189 & 2198.84 & 497 & 355 & 0.0 \\
\hline 59850 & 77.8834 & 155.8126 & 2199.27 & 498 & 355 & 0.0 \\
\hline 60000 & 77.8836 & 155.8063 & 2199.49 & 499 & 355 & 63646.9 \\
\hline 60150 & 77.8838 & 155.8000 & 2199.96 & 500 & 355 & 0.0 \\
\hline 60300 & 77.8840 & 155.7937 & 2200.35 & 501 & 355 & 0.0 \\
\hline 60450 & 77.8842 & 155.7874 & 2200.70 & 502 & 355 & 63638.9 \\
\hline 60600 & 77.8844 & 155.7811 & 2201.09 & 503 & 355 & 0.0 \\
\hline 60750 & 77.8847 & 155.7747 & 2201.32 & 504 & 355 & 0.0 \\
\hline 60900 & 77.8849 & 155.7684 & 2201.99 & 505 & 355 & 63624.6 \\
\hline 61050 & 77.8851 & 155.7621 & 2202.47 & 506 & 355 & 0.0 \\
\hline 61200 & 77.8853 & 155.7558 & 2202.91 & 507 & 355 & 0.0 \\
\hline 61350 & 77.8855 & 155.7495 & 2203.52 & 508 & 355 & 63617.8 \\
\hline 61500 & 77.8858 & 155.7431 & 2203.92 & 509 & 355 & 0.0 \\
\hline 61650 & 77.8860 & 155.7368 & 2204.69 & 510 & 355 & 0.0 \\
\hline 61800 & 77.8862 & 155.7305 & 2205.35 & 511 & 355 & 63595.9 \\
\hline 61950 & 77.8864 & 155.7242 & 2206.19 & 512 & 355 & 0.0 \\
\hline 62100 & 77.8866 & 155.7179 & 2206.95 & 513 & 355 & 0.0 \\
\hline 62250 & 77.8868 & 155.7116 & 2207.76 & 514 & 355 & 63600.5 \\
\hline
\end{tabular}




\begin{tabular}{|c|c|c|c|c|c|c|}
\hline Distance (m) & $\begin{array}{l}\text { Flag Loc. } \\
\text { Latitude ('S) }\end{array}$ & $\begin{array}{c}\text { Flag Loc. } \\
\text { Longitude }\left({ }^{\circ} \mathrm{E}\right)\end{array}$ & Elevation (m) & $\begin{array}{c}\text { Shot } \\
\text { Number }\end{array}$ & $\begin{array}{c}\text { Julian } \\
\text { Day }\end{array}$ & $\begin{array}{l}\text { Total Mag. } \\
\text { Field (nT) }\end{array}$ \\
\hline 62400 & 77.8871 & 55.7052 & 2208.77 & $\overline{515}$ & 355 & $\overline{0.0}$ \\
\hline 62550 & 77.8873 & 155.6989 & 2209.70 & 516 & 355 & 0.0 \\
\hline 62700 & 77.8875 & 155.6926 & 2210.47 & 517 & 355 & 63616.7 \\
\hline 62850 & 77.8877 & 155.6863 & 2211.16 & 518 & 355 & 0.0 \\
\hline 63000 & 77.8879 & 155.6800 & 2211.96 & 519 & 355 & 63606.3 \\
\hline 63150 & 77.8881 & 155.6736 & 2212.53 & 520 & 355 & 63601.1 \\
\hline 63300 & 77.8884 & 155.6673 & 2213.08 & 521 & 355 & 0.0 \\
\hline 63450 & 77.8886 & 155.6610 & 2213.53 & 522 & 355 & 0.0 \\
\hline 63600 & 77.8888 & 155.6547 & 2213.78 & 523 & 355 & 63596.6 \\
\hline 63750 & 77.8890 & 155.6484 & 2214.00 & 524 & 355 & 0.0 \\
\hline 63900 & 77.8892 & 155.6421 & 2214.06 & 525 & 355 & 0.0 \\
\hline 64050 & 77.8894 & 155.6357 & 2214.04 & 526 & 355 & 63592.1 \\
\hline 64200 & 77.8897 & 155.6294 & 2214.00 & 527 & 355 & 0.0 \\
\hline 64350 & 77.8899 & 155.6231 & 2214.38 & 528 & 355 & 63597.5 \\
\hline 64500 & 77.8901 & 155.6168 & 2214.39 & 529 & 355 & 63592.4 \\
\hline 64650 & 77.8903 & 155.6105 & 2214.29 & 530 & 355 & 0.0 \\
\hline 64800 & 77.8905 & 155.6042 & 2214.43 & 531 & 355 & 0.0 \\
\hline 64950 & 77.8908 & 155.5978 & 2214.57 & 532 & 355 & 63582.9 \\
\hline 65100 & 77.8910 & 155.5915 & 2214.85 & 533 & 355 & 0.0 \\
\hline 65250 & 77.8912 & 155.5852 & 2215.14 & 534 & 355 & 0.0 \\
\hline 65400 & 77.8914 & 155.5789 & 2215.41 & 535 & 355 & 63558.1 \\
\hline 65550 & 77.8916 & 155.5726 & 2215.27 & 536 & 355 & 0.0 \\
\hline 65700 & 77.8918 & 155.5663 & 2215.00 & 537 & 355 & 0.0 \\
\hline 65850 & 77.8921 & 155.5599 & 2214.43 & 538 & 355 & 63529.1 \\
\hline 66000 & 77.8923 & 155.5536 & 2213.36 & 539 & 355 & 0.0 \\
\hline 66150 & 77.8925 & 155.5473 & 2212.42 & 540 & 355 & 0.0 \\
\hline 66300 & 77.8927 & 155.5410 & 2211.29 & 541 & 355 & 63511.6 \\
\hline 66450 & 77.8929 & 155.5347 & 2210.69 & 542 & 355 & 0.0 \\
\hline 66600 & 77.8932 & 155.5283 & 2210.13 & 543 & 355 & 0.0 \\
\hline 66750 & 77.8934 & 155.5220 & 2209.90 & 544 & 355 & 63509.5 \\
\hline 66900 & 77.8936 & 155.5157 & 2210.01 & 545 & 355 & 0.0 \\
\hline 67050 & 77.8938 & 155.5094 & 2210.53 & 546 & 355 & 0.0 \\
\hline 67200 & 77.8940 & 155.5031 & 2211.36 & 547 & 355 & 63532.2 \\
\hline 67350 & 77.8942 & 155.4967 & 2212.14 & 548 & 355 & 0.0 \\
\hline 67500 & 77.8945 & 155.4904 & 2213.06 & 549 & 355 & 0.0 \\
\hline 67650 & 77.8947 & 155.4841 & 2214.07 & 550 & 355 & 63540.6 \\
\hline 67800 & 77.8949 & 155.4778 & 2215.05 & 551 & 355 & 0.0 \\
\hline 67950 & 77.8951 & 155.4714 & 2216.21 & 552 & 355 & 63535.0 \\
\hline 68100 & 77.8953 & 155.4651 & 2217.34 & 553 & 355 & 63534.5 \\
\hline 68250 & 77.8956 & 155.4588 & 2218.79 & 554 & 355 & 0.0 \\
\hline 68400 & 77.8958 & 155.4525 & 2220.01 & 555 & 355 & 0.0 \\
\hline 68550 & 77.8960 & 155.4462 & 2221.57 & 556 & 355 & 63505.4 \\
\hline 68700 & 77.8962 & 155.4398 & 2223.31 & 557 & 355 & 0.0 \\
\hline 68850 & 77.8964 & 155.4335 & 2225.04 & 558 & 355 & 0.0 \\
\hline 69000 & 77.8966 & 155.4272 & 2226.63 & 559 & 355 & 63493.6 \\
\hline 69150 & 77.8969 & 155.4209 & 2228.36 & 560 & 355 & 0.0 \\
\hline 69300 & 77.8971 & 155.4146 & 2229.89 & 561 & 355 & 0.0 \\
\hline 69450 & 77.8973 & 155.4082 & 2231.66 & 562 & 356 & 63430.1 \\
\hline 69600 & 77.8975 & 155.4019 & 2233.04 & 563 & 356 & 0.0 \\
\hline 69750 & 77.8977 & 155.3956 & 2234.30 & 564 & 356 & 0.0 \\
\hline 69900 & 77.8980 & 155.3893 & 2235.36 & 565 & 356 & 63312.5 \\
\hline 70050 & 77.8982 & 155.3829 & 2236.43 & 566 & 356 & 0.0 \\
\hline
\end{tabular}




\begin{tabular}{|c|c|c|c|c|c|c|}
\hline Distance (m) & $\begin{array}{c}\text { Flag Loc. } \\
\text { Latitude }\left({ }^{\circ} \mathbf{S}\right)\end{array}$ & $\begin{array}{c}\text { Flag Loc. } \\
\text { Longitude ( } \\
\end{array}$ & Elevation (m) & $\begin{array}{c}\text { Shot } \\
\text { Number }\end{array}$ & $\begin{array}{c}\text { Julian } \\
\text { Day }\end{array}$ & $\begin{array}{l}\text { Total Mag. } \\
\text { Field (nT) }\end{array}$ \\
\hline 70200 & 77.8984 & 155.3766 & 2237.26 & 567 & 356 & $\overline{0.0}$ \\
\hline 70350 & 77.8986 & 155.3703 & 2237.78 & 568 & 356 & 63278.5 \\
\hline 70500 & 77.8988 & 155.3640 & 2238.38 & 569 & 356 & 0.0 \\
\hline 70650 & 77.8990 & 155.3577 & 2238.68 & 570 & 356 & 0.0 \\
\hline 70800 & 77.8993 & 155.3513 & 2239.11 & 571 & 356 & 63285.3 \\
\hline 70950 & 77.8995 & 155.3450 & 2239.30 & 572 & 356 & 0.0 \\
\hline 71100 & 77.8997 & 155.3387 & 2239.73 & 573 & 356 & 0.0 \\
\hline 71250 & 77.8999 & 155.3324 & 2239.89 & 574 & 356 & 63299.4 \\
\hline 71400 & 77.9001 & 155.3260 & 2240.29 & 575 & 356 & 0.0 \\
\hline 71550 & 77.9004 & 155.3197 & 2240.33 & 576 & 356 & 63307.3 \\
\hline 71700 & 77.9006 & 155.3134 & 2240.42 & 577 & 356 & 63323.5 \\
\hline 71850 & 77.9008 & 155.3071 & 2240.63 & 578 & 356 & 0.0 \\
\hline 72000 & 77.9010 & 155.3007 & 2240.59 & 579 & 356 & 0.0 \\
\hline 72150 & 77.9012 & 155.2944 & 2240.72 & 580 & 356 & 63332.0 \\
\hline 72300 & 77.9014 & 155.2881 & 2240.44 & 581 & 356 & 0.0 \\
\hline 72450 & 77.9016 & 155.2817 & 2240.46 & 582 & 356 & 0.0 \\
\hline 72600 & 77.9018 & 155.2754 & 2240.26 & 583 & 356 & 63361.1 \\
\hline 72750 & 77.9020 & 155.2690 & 2240.22 & 584 & 356 & 0.0 \\
\hline 72900 & 77.9022 & 155.2627 & 2240.11 & 585 & 356 & 0.0 \\
\hline 73050 & 77.9024 & 155.2564 & 2239.96 & 586 & 356 & 63387.0 \\
\hline 73200 & 77.9026 & 155.2500 & 2239.33 & 587 & 356 & 0.0 \\
\hline 73350 & 77.9028 & 155.2437 & 2239.02 & 588 & 356 & 0.0 \\
\hline 73500 & 77.9030 & 155.2374 & 2238.88 & 589 & 356 & 63384.7 \\
\hline 73650 & 77.9032 & 155.2310 & 2237.95 & 590 & 356 & 63375.3 \\
\hline 73800 & 77.9034 & 155.2247 & 2237.64 & 591 & 356 & 0.0 \\
\hline 73950 & 77.9036 & 155.2184 & 2237.30 & 592 & 356 & 63384.6 \\
\hline 74100 & 77.9038 & 155.2120 & 2236.99 & 593 & 356 & 0.0 \\
\hline 74250 & 77.9040 & 155.2057 & 2236.62 & 594 & 356 & 0.0 \\
\hline 74400 & 77.9042 & 155.1993 & 2236.33 & 595 & 356 & 63413.6 \\
\hline 74550 & 77.9044 & 155.1930 & 2235.98 & 596 & 356 & 0.0 \\
\hline 74700 & 77.9046 & 155.1867 & 2235.71 & 597 & 356 & 0.0 \\
\hline 74850 & 77.9048 & 155.1803 & 2235.14 & 0 & 361 & 63408.3 \\
\hline 75000 & 77.9050 & 155.1740 & 2234.49 & 0 & 361 & 0.0 \\
\hline 75150 & 77.9052 & 155.1677 & 2233.28 & 0 & 361 & 0.0 \\
\hline 75300 & 77.9054 & 155.1613 & 2233.05 & o & 361 & 63428.0 \\
\hline 75450 & 77.9056 & 155.1550 & 2232.44 & 이 & 361 & 0.0 \\
\hline 75600 & 77.9058 & 155.1487 & 2231.99 & 이 & 361 & 0.0 \\
\hline 75750 & 77.9060 & 155.1423 & 2231.70 & 이 & 361 & 63419.1 \\
\hline 75900 & 77.9062 & 155.1360 & 2231.67 & 0 & 361 & 0.0 \\
\hline 76050 & 77.9064 & 155.1296 & 2231.95 & 0 & 361 & 0.0 \\
\hline 76200 & 77.9066 & 155.1233 & 2232.61 & 0 & 361 & 63413.1 \\
\hline 76350 & 77.9068 & 155.1170 & 2233.32 & 이 & 361 & 0.0 \\
\hline 76500 & 77.9070 & 155.1106 & 2234.17 & 0 & 361 & 0.0 \\
\hline 76650 & 77.9072 & 155.1043 & 2235.11 & 이 & 361 & 63410.4 \\
\hline 76800 & 77.9074 & 155.0979 & 2236.08 & 이 & 361 & 0.0 \\
\hline 76950 & 77.9076 & 155.0916 & 2236.67 & 이 & 361 & 0.0 \\
\hline 77100 & 77.9078 & 155.0853 & 2238.01 & 0 & 361 & 63413.7 \\
\hline 77250 & 77.9080 & 155.0789 & 2239.11 & 0 & 361 & 0.0 \\
\hline 77400 & 77.9082 & 155.0726 & 2240.11 & 이 & 361 & 0.0 \\
\hline 77550 & 77.9084 & 155.0663 & 2241.35 & 이 & 361 & 63401.2 \\
\hline 77700 & 77.9086 & 155.0599 & 2242.47 & 0 & 361 & 0.0 \\
\hline 77850 & 77.9089 & 155.0535 & 2243.47 & 이 & 361| & 63388.7 \\
\hline
\end{tabular}




\begin{tabular}{|c|c|c|c|c|c|c|}
\hline Distance $(\mathrm{m})$ & $\begin{array}{c}\text { Flag Loc. } \\
\text { Latitude ( }{ }^{\circ} \text { ) }\end{array}$ & $\begin{array}{c}\text { Flag Loc. } \\
\left.\text { Longitude ( }{ }^{\circ} E\right)\end{array}$ & Elevation (m) & $\begin{array}{c}\text { Shot } \\
\text { Number }\end{array}$ & $\begin{array}{c}\text { Julian } \\
\text { Day }\end{array}$ & $\begin{array}{l}\text { Total Mag. } \\
\text { Field (nT) }\end{array}$ \\
\hline 78000 & 77.9091 & 755.0472 & 2244.78 & $\overline{0}$ & 361 & 0.0 \\
\hline 78150 & 77.9093 & 155.0409 & 2244.78 & 0 & 361 & 0.0 \\
\hline 78300 & 77.9095 & 155.0345 & 2244.88 & 0 & 361 & 63373.9 \\
\hline 78450 & 77.9097 & 155.0282 & 2244.98 & 0 & 361 & 0.0 \\
\hline 78600 & 77.9099 & 155.0219 & 2244.64 & 0 & 361 & 0.0 \\
\hline 78750 & 77.9101 & 155.0155 & 2244.51 & 0 & 361 & 63389.2 \\
\hline 78900 & 77.9103 & 155.0092 & 2244.06 & 0 & 361 & 0.0 \\
\hline 79050 & 77.9105 & 155.0028 & 2243.94 & 0 & 361 & 0.0 \\
\hline 79200 & 77.9107 & 154.9965 & 2244.23 & 0 & 361 & 63422.3 \\
\hline 79350 & 77.9109 & 154.9902 & 2245.01 & 0 & 361 & 0.0 \\
\hline 79500 & 77.9111 & 154.9838 & 2245.91 & 0 & 361 & 0.0 \\
\hline 79650 & 77.9113 & 154.9775 & 2247.16 & 0 & 361 & 63456.7 \\
\hline 79800 & 77.9115 & 154.9711 & 2248.37 & 0 & 361 & 0.0 \\
\hline 79950 & 77.9117 & 154.9648 & 2250.04 & 0 & 361 & 63466.0 \\
\hline 80100 & 77.9119 & 154.9584 & 2251.53 & 0 & 361 & 0.0 \\
\hline 80250 & 77.9121 & 154.9521 & 2253.00 & 0 & 361 & 0.0 \\
\hline 80400 & 77.9123 & 154.9458 & 2254.36 & 0 & 361 & 63470.3 \\
\hline 80550 & 77.9125 & 154.9394 & 2255.43 & 0 & 361 & 0.0 \\
\hline 80700 & 77.9127 & 154.9331 & 2256.52 & 0 & 361 & 0.0 \\
\hline 80850 & 77.9129 & 154.9267 & 2257.29 & 0 & 361 & 63466.4 \\
\hline 81000 & 77.9145 & 154.8760 & 2257.09 & 0 & 361 & 0.0 \\
\hline 81150 & 77.9133 & 154.9140 & 2258.61 & 0 & 361 & 63463.5 \\
\hline 81300 & 77.9135 & 154.9077 & 2258.87 & 0 & 361 & 0.0 \\
\hline 81450 & 77.9137 & 154.9014 & 2258.75 & 0 & 361 & 0.0 \\
\hline 81600 & 77.9139 & 154.8950 & 2258.61 & 0 & 361 & 63452.9 \\
\hline 81750 & 77.9141 & 154.8887 & 2258.16 & 0 & 361 & 0.0 \\
\hline 81900 & 77.9143 & 154.8823 & 2257.71 & 0 & 361 & 0.0 \\
\hline 82200 & 77.9147 & 154.8697 & 2256.35 & 0 & 361 & 0.0 \\
\hline 82350 & 77.9149 & 154.8633 & 2255.65 & 0 & 361 & 0.0 \\
\hline 82500 & 77.9151 & 154.8570 & 2255.03 & 0 & 361 & 63443.6 \\
\hline 82650 & 77.9153 & 154.8506 & 2254.27 & 0 & 361 & 0.0 \\
\hline 82800 & 77.9155 & 154.8443 & 2253.53 & 0 & 361 & 0.0 \\
\hline 82950 & 77.9157 & 154.8379 & 2252.91 & 0 & 361 & 63438.9 \\
\hline 83100 & 77.9159 & 154.8316 & 2252.40 & 0 & 361 & 0.0 \\
\hline 83250 & 77.9161 & 154.8253 & 2251.94 & 0 & 361 & 0.0 \\
\hline 83400 & 77.9163 & 154.8189 & 2251.39 & 0 & 361 & 63431.6 \\
\hline 83550 & 77.9165 & 154.8126 & 2250.98 & 0 & 361 & 0.0 \\
\hline 83700 & 77.9167 & 154.8062 & 2250.64 & 0 & 361 & 0.0 \\
\hline 83850 & 77.9169 & 154.7999 & 2250.58 & 0 & 361 & 63430.4 \\
\hline 84000 & 77.9171 & 154.7935 & 2250.26 & 0 & 361 & 0.0 \\
\hline 84150 & 77.9173 & 154.7872 & 2250.19 & 0 & 361 & 0.0 \\
\hline 84300 & 77.9175 & 154.7809 & 2249.94 & 0 & 361 & 63428.4 \\
\hline 84450 & 77.9177 & 154.7745 & 2249.53 & 0 & 361 & 0.0 \\
\hline 84600 & 77.9179 & 154.7681 & 2249.96 & 0 & 361 & 63426.8 \\
\hline 84750 & 77.9181 & 154.7618 & 2250.15 & 0 & 361 & 0.0 \\
\hline 84900 & 77.9183 & 154.7555 & 2250.30 & 0 & 361 & 0.0 \\
\hline 8505 이 & 77.9185 & 154.7491 & 2250.44 & 0 & 361 & 63432.4 \\
\hline 85200 & 77.9187 & 154.7428 & 2250.65 & 0 & 361 & 0.0 \\
\hline 85350 & 77.9189 & 154.7364 & 2250.92 & 0 & 361 & 0.0 \\
\hline 85500 & 77.9191 & 154.7301 & 2251.28 & 0 & 361 & 63431.7 \\
\hline 85650 & 77.9193 & 154.7238 & 2251.54 & 0 & 361 & 0.0 \\
\hline 85800 & 77.9195 & 154.7174 & 2251.76 & 0 & 361 & 0.0 \\
\hline
\end{tabular}




\begin{tabular}{|c|c|c|c|c|c|c|}
\hline Distance (m) & $\begin{array}{l}\text { Flag Loc. } \\
\left.\text { Latitude ( }{ }^{\circ} \mathrm{S}\right)\end{array}$ & $\begin{array}{c}\text { Flag Loc. } \\
\text { Longitude }\left({ }^{\circ} E\right)\end{array}$ & Elevation (m) & $\begin{array}{c}\text { Shot } \\
\text { Number }\end{array}$ & $\begin{array}{c}\text { Julian } \\
\text { Day }\end{array}$ & $\begin{array}{l}\text { Total Mag. } \\
\text { Field (nT) }\end{array}$ \\
\hline $8 \overline{85950}$ & 77.9197 & 154.7111 & 2257.70 & $\overline{0}$ & 361 & 63429.4 \\
\hline 86100 & 77.9199 & 154.7047 & 2251.77 & 0 & 361 & 0.0 \\
\hline 86250 & 77.9202 & 154.6984 & 2251.85 & 0 & 361 & 0.0 \\
\hline 86400 & 77.9203 & 154.6920 & 2251.95 & 0 & 361 & 63429.5 \\
\hline 86550 & 77.9205 & 154.6857 & 2252.09 & 0 & 361 & 0.0 \\
\hline 86700 & 77.9208 & 154.6793 & 2252.18 & 0 & 361 & 63433.7 \\
\hline 86850 & 77.9210 & 154.6730 & 2252.07 & 0 & 361 & 0.0 \\
\hline 87000 & 77.9212 & 154.6666 & 2252.08 & 0 & 361 & 0.0 \\
\hline 87150 & 77.9214 & 154.6603 & 2251.92 & 0 & 361 & 63440.8 \\
\hline 87300 & 77.9216 & 154.6539 & 2251.77 & 0 & 361 & 0.0 \\
\hline 87450 & 77.9218 & 154.6476 & 2251.91 & 0 & 361 & 0.0 \\
\hline 87600 & 77.9220 & 154.6413 & 2251.70 & 0 & 361 & 63444.6 \\
\hline 87750 & 77.9222 & 154.6349 & 2251.57 & 0 & 361 & 0.0 \\
\hline 87900 & 77.9224 & 154.6286 & 2251.58 & 0 & 361 & 0.0 \\
\hline 88050 & 77.9226 & 154.6222 & 2251.34 & 0 & 361 & 63449.6 \\
\hline 88200 & 77.9228 & 154.6159 & 2251.26 & 0 & 361 & 0.0 \\
\hline 88350 & 77.9230 & 154.6095 & 2251.32 & 0 & 361 & 0.0 \\
\hline 88500 & 77.9232 & 154.6032 & 2251.01 & 0 & 361 & 63453.9 \\
\hline 88650 & 77.9234 & 154.5968 & 2250.97 & 0 & 361 & 0.0 \\
\hline 88800 & 77.9236 & 154.5905 & 2250.80 & 0 & 361 & 63457.8 \\
\hline 88950 & 77.9238 & 154.5841 & 2250.60 & 0 & 361 & 0.0 \\
\hline 89100 & 77.9240 & 154.5778 & 2250.66 & 0 & 361 & 0.0 \\
\hline 89250 & 77.9242 & 154.5714 & 2250.60 & 0 & 361 & 63463.2 \\
\hline 89400 & 77.9244 & 154.5651 & 2250.49 & 0 & 361 & 0.0 \\
\hline 89550 & 77.9246 & 154.5587 & 2250.39 & 0 & 361 & 0.0 \\
\hline 89700 & 77.9248 & 154.5524 & 2250.56 & 0 & 361 & 63466.4 \\
\hline 89850 & 77.9250 & 154.5460 & 2250.58 & 0 & 361 & 0.0 \\
\hline 90000 & 77.9252 & 154.5397 & 2250.70 & 0 & 361 & 0.0 \\
\hline 90150 & 77.9254 & 154.5333 & 2250.78 & 700 & 361 & 63467.3 \\
\hline 90300 & 77.9256 & 154.5270 & 2251.07 & 701 & 361 & 0.0 \\
\hline 90450 & 77.9258 & 154.5206 & 2250.84 & 702 & 361 & 0.0 \\
\hline 90600 & 77.9260 & 154.5143 & 2250.75 & 703 & 362 & 0.0 \\
\hline 90750 & 77.9262 & 154.5079 & 2251.14 & 704 & 362 & 0.0 \\
\hline 90900 & 77.9264 & 154.5016 & 2251.33 & 705 & 362 & 63550.2 \\
\hline 91050 & 77.9266 & 154.4952 & 2251.43 & 706 & 362 & 63547.6 \\
\hline 91200 & 77.9268 & 154.4889 & 2251.66 & 707 & 362 & 0.0 \\
\hline 91350 & 77.9270 & 154.4825 & 2251.91 & 708 & 362 & 0.0 \\
\hline 91500 & 77.9272 & 154.4762 & 2252.21 & 709 & 362 & 63543.2 \\
\hline 91650 & 77.9274 & 154.4698 & 2252.63 & 710 & 362 & 0.0 \\
\hline 91800 & 77.9276 & 154.4635 & 2252.68 & 711 & 362 & 0.0 \\
\hline 91950 & 77.9278 & 154.4571 & 2252.71 & 712 & 362 & 63527.5 \\
\hline 92100 & 77.9280 & 154.4508 & 2252.81 & 713 & 362 & 0.0 \\
\hline 92250 & 77.9282 & 154.4444 & 2252.97 & 714 & 362 & 0.0 \\
\hline 92400 & 77.9284 & 154.4381 & 2252.96 & 715 & 362 & 63507.0 \\
\hline 92550 & 77.9286 & 154.4317 & 2253.24 & 716 & 362 & 0.0 \\
\hline 92700 & 77.9288 & 154.4254 & 2253.65 & 717 & 362 & 0.0 \\
\hline 92850 & 77.9290 & 154.4190 & 2254.30 & 718 & 362 & 63453.8 \\
\hline 93000 & 77.9292 & 154.4127 & 2255.06 & 719 & 362 & 63425.4 \\
\hline 93150 & 77.9294 & 154.4063 & 2255.82 & 720 & 362 & 0.0 \\
\hline 93300 & 77.9296 & 154.4000 & 2256.54 & 721 & 362 & 63468.6 \\
\hline 93450 & 77.9298 & 154.3936 & 2257.64 & 722 & 362 & 0.0 \\
\hline 93600 & 77.9300 & 154.3873 & 2258.37 & 723 & 362 & 0.0 \\
\hline
\end{tabular}




\begin{tabular}{|c|c|c|c|c|c|c|}
\hline Distance (m) & $\begin{array}{c}\text { Flag Loc. } \\
\text { Latitude ('S) }\end{array}$ & $\begin{array}{c}\text { Flag Loc. } \\
\text { Longitude }\left({ }^{\circ} \mathrm{E}\right)\end{array}$ & Elevation $(\mathrm{m})$ & $\begin{array}{c}\text { Shot } \\
\text { Number }\end{array}$ & $\begin{array}{c}\text { Julian } \\
\text { Day }\end{array}$ & $\begin{array}{l}\text { Total Mag. } \\
\text { Field (nT) }\end{array}$ \\
\hline$\overline{93750}$ & 77.9302 & $\overline{154.3809}$ & 2259.37 & 724 & 362 & 63506.9 \\
\hline 93900 & 77.9304 & 154.3746 & 2260.08 & 725 & 362 & 0.0 \\
\hline 94050 & 77.9306 & 154.3682 & 2260.57 & 726 & 362 & 0.0 \\
\hline 94200 & 77.9308 & 154.3619 & 2261.38 & 727 & 362 & 63519.4 \\
\hline 94350 & 77.9310 & 154.3555 & 2261.80 & 728 & 362 & 0.0 \\
\hline 94500 & 77.9312 & 154.3492 & 2262.04 & 729 & 362 & 0.0 \\
\hline 94650 & 77.9314 & 154.3428 & 2262.49 & 730 & 362 & 63476.7 \\
\hline 94800 & 77.9316 & 154.3364 & 2262.91 & 731 & 362 & 63448.5 \\
\hline 94950 & 77.9318 & 154.3301 & 2263.15 & 732 & 362 & 0.0 \\
\hline 95100 & 77.9320 & 154.3237 & 2263.30 & 733 & 362 & 63450.7 \\
\hline 95250 & 77.9322 & 154.3174 & 2263.42 & 734 & 362 & 0.0 \\
\hline 95400 & 77.9324 & 154.3110 & 2263.54 & 735 & 362 & 0.0 \\
\hline 95550 & 77.9326 & 154.3047 & 2263.53 & 736 & 362 & 63463.4 \\
\hline 95700 & 77.9328 & 154.2983 & 2263.35 & 737 & 362 & 0.0 \\
\hline 95850 & 77.9330 & 154.2920 & 2263.51 & 738 & 362 & 0.0 \\
\hline 96000 & 77.9332 & 154.2856 & 2263.49 & 739 & 362 & 63467.7 \\
\hline 96150 & 77.9334 & 154.2793 & 2263.34 & 740 & 362 & 0.0 \\
\hline 96300 & 77.9336 & 154.2729 & 2263.06 & 741 & 362 & 0.0 \\
\hline 96450 & 77.9338 & 154.2666 & 2263.07 & 742 & 362 & 63461.6 \\
\hline 96600 & 77.9340 & 154.2602 & 2262.83 & 743 & 362 & 0.0 \\
\hline 96750 & 77.9342 & 154.2539 & 2262.43 & 744 & 362 & 0.0 \\
\hline 96900 & 77.9344 & 154.2475 & 2262.60 & 745 & 362 & 63470.5 \\
\hline 97050 & 77.9346 & 154.2411 & 2262.34 & 746 & 362 & 0.0 \\
\hline 97200 & 77.9348 & 154.2348 & 2262.03 & 747 & 362 & 0.0 \\
\hline 97350 & 77.9350 & 154.2284 & 2261.97 & 748 & 362 & 63487.8 \\
\hline 97500 & 77.9352 & 154.2221 & 2261.81 & 749 & 362 & 0.0 \\
\hline 97650 & 77.9354 & 154.2157 & 2261.76 & 750 & 362 & 0.0 \\
\hline 97800 & 77.9356 & 154.2094 & 2261.63 & 751 & 362 & 63496.2 \\
\hline 97950 & 77.9358 & 154.2030 & 2261.45 & 752 & 362 & 0.0 \\
\hline 98100 & 77.9360 & 154.1967 & 2261.36 & 753 & 362 & 0.0 \\
\hline 98250 & 77.9362 & 154.1903 & 2261.32 & 754 & 362 & 63493.0 \\
\hline 98400 & 77.9364 & 154.1840 & 2261.17 & 755 & 362 & 0.0 \\
\hline 98550 & 77.9366 & 154.1776 & 2261.24 & 756 & 362 & 0.0 \\
\hline 98700 & 77.9368 & 154.1713 & 2261.45 & 757 & 362 & 63487.7 \\
\hline 98850 & 77.9370 & 154.1649 & 2261.66 & 758 & 362 & 0.0 \\
\hline 99000 & 77.9372 & 154.1585 & 2262.54 & 759 & 362 & 63491.1 \\
\hline 99150 & 77.9374 & 154.1522 & 2263.03 & 760 & 362 & 63486.2 \\
\hline 99300 & 77.9376 & 154.1458 & 2263.72 & 761 & 362 & 0.0 \\
\hline 99450 & 77.9378 & 154.1395 & 2264.29 & 762 & 362 & 0.0 \\
\hline 99600 & 77.9380 & 154.1331 & 2264.77 & 763 & 362 & 63483.3 \\
\hline 99750 & 77.9382 & 154.1268 & 2265.22 & 764 & 362 & 0.0 \\
\hline 99900 & 77.9384 & 154.1204 & 2265.59 & 765 & 362 & 0.0 \\
\hline 100050 & 77.9386 & 154.1140 & 2266.16 & 766 & 362 & 63481.8 \\
\hline 100200 & 77.9388 & 154.1077 & 2266.47 & 767 & 362 & 0.0 \\
\hline 100350 & 77.9390 & 154.1013 & 2266.86 & 768 & 362 & 0.0 \\
\hline 100500 & 77.9392 & 154.0950 & 2267.16 & 769 & 362 & 63484.2 \\
\hline 100650 & 77.9394 & 154.0886 & 2267.63 & 770 & 362 & 0.0 \\
\hline 100800 & 77.9396 & 154.0823 & 2267.90 & 771 & 362 & 0.0 \\
\hline 100950 & 77.9398 & 154.0759 & 2268.11 & 772 & 362 & 63483.4 \\
\hline 101100 & 77.9400 & 154.0695 & 2268.53 & 773 & 362 & 63484.4 \\
\hline 101250 & 77.9402 & 154.0632 & 2268.69 & 774 & 362 & 0.0 \\
\hline 101400 & 77.9404 & 154.0568 & 2269.09 & 775 & 362 & 63477.2 \\
\hline
\end{tabular}




\begin{tabular}{|c|c|c|c|c|c|c|}
\hline Distance (m) & $\begin{array}{l}\text { Flag Loc. } \\
\left.\text { Latitude ( }{ }^{\circ} \mathrm{S}\right)\end{array}$ & $\begin{array}{c}\text { Flag Loc. } \\
\text { Longitude }\left({ }^{\circ} \mathrm{E}\right)\end{array}$ & Elevation (m) & $\begin{array}{l}\text { Shot } \\
\text { Number }\end{array}$ & $\begin{array}{l}\text { Julian } \\
\text { Day }\end{array}$ & $\begin{array}{l}\text { Total Mag. } \\
\text { Field (nT) }\end{array}$ \\
\hline 701550 & 77.9406 & 154.0505 & 2269.42 & 776 & $\overline{362}$ & 0.0 \\
\hline 101700 & 77.9408 & 154.0441 & 2269.65 & 777 & 362 & 0.0 \\
\hline 101850 & 77.9410 & 154.0378 & 2269.91 & 778 & 362 & 63482.7 \\
\hline 102000 & 77.9412 & 154.0314 & 2270.14 & 779 & 362 & 0.0 \\
\hline 102150 & 77.9414 & 154.0250 & 2270.15 & 780 & 362 & 0.0 \\
\hline 102300 & 77.9416 & 154.0187 & 2270.48 & 781 & 362 & 63488.8 \\
\hline 102450 & 77.9418 & 154.0123 & 2270.73 & 782 & 362 & 0.0 \\
\hline 102600 & 77.9420 & 154.0060 & 2270.54 & 783 & 362 & 0.0 \\
\hline 102750 & 77.9422 & 153.9996 & 2270.74 & 784 & 362 & 63498.9 \\
\hline 102900 & 77.9424 & 153.9933 & 2271.22 & 785 & 362 & 0.0 \\
\hline 103050 & 77.9426 & 153.9869 & 2271.04 & 786 & 362 & 0.0 \\
\hline 103200 & 77.9428 & 153.9805 & 2271.38 & 787 & 362 & 63500.5 \\
\hline 103350 & 77.9430 & 153.9741 & 2271.46 & 788 & 362 & 0.0 \\
\hline 103500 & 77.9432 & 153.9678 & 2271.60 & 789 & 362 & 0.0 \\
\hline 103650 & 77.9433 & 153.9614 & 2271.87 & 790 & 362 & 63509.8 \\
\hline 103800 & 77.9435 & 153.9550 & 2272.06 & 791 & 362 & 0.0 \\
\hline 103950 & 77.9437 & 153.9486 & 2272.28 & 792 & 362 & 0.0 \\
\hline 104100 & 77.9438 & 153.9422 & 2272.38 & 793 & 362 & 63517.1 \\
\hline 104250 & 77.9440 & 153.9359 & 2272.69 & 794 & 362 & 0.0 \\
\hline 104400 & 77.9442 & 153.9295 & 2273.01 & 795 & 362 & 0.0 \\
\hline 104550 & 77.9443 & 153.9231 & 2273.23 & 796 & 362 & 63526.6 \\
\hline 104700 & 77.9445 & 153.9167 & 2273.30 & 797 & 362 & 0.0 \\
\hline 104850 & 77.9447 & 153.9104 & 2273.68 & 798 & 362 & 0.0 \\
\hline 105000 & 77.9448 & 153.9040 & 2273.66 & 799 & 362 & 63531.0 \\
\hline 105150 & 77.9450 & 153.8976 & 2273.94 & 800 & 362 & 0.0 \\
\hline 105300 & 77.9452 & 153.8912 & 2273.95 & 801 & 362 & 63535.1 \\
\hline 105450 & 77.9453 & 153.8848 & 2273.97 & 802 & 1 & 63632.0 \\
\hline 105600 & 77.9455 & 153.8784 & 2274.18 & 803 & 1 & 0.0 \\
\hline 105750 & 77.9457 & 153.8720 & 2274.51 & 804 & 1 & 0.0 \\
\hline 105900 & 77.9458 & 153.8657 & 2274.70 & 805 & 1 & 63642.3 \\
\hline 106050 & 77.9460 & 153.8593 & 2275.02 & 806 & 1 & 0.0 \\
\hline 106200 & 77.9462 & 153.8529 & 2275.32 & 807 & 1 & 0.0 \\
\hline 106350 & 77.9464 & 153.8465 & 2275.60 & 808 & 1 & 63648.2 \\
\hline 106500 & 77.9465 & 153.8401 & 2275.78 & 809 & 1 & 0.0 \\
\hline 106650 & 77.9467 & 153.8338 & 2276.11 & 810 & 1 & 0.0 \\
\hline 106800 & 77.9468 & 153.8274 & 2276.36 & 811 & 1 & 63657.1 \\
\hline 106950 & 77.9470 & 153.8210 & 2276.82 & 812 & 1 & 0.0 \\
\hline 107100 & 77.9472 & 153.8146 & 2277.11 & 813 & 1 & 0.0 \\
\hline 107250 & 77.9473 & 153.8082 & 2277.49 & 814 & 1 & 63651.4 \\
\hline 107400 & 77.9475 & 153.8018 & 2277.30 & 815 & 1 & 63648.0 \\
\hline 107550 & 77.9477 & 153.7955 & 2277.60 & 816 & 1 & 0.0 \\
\hline 107700 & 77.9479 & 153.7891 & 2277.93 & 817 & 1 & 63648.2 \\
\hline 107850 & 77.9480 & 153.7827 & 2278.18 & 818 & 1 & 0.0 \\
\hline 108000 & 77.9482 & 153.7763 & 2278.60 & 819 & 1 & 0.0 \\
\hline 108150 & 77.9484 & 153.7699 & 2278.99 & 820 & 1 & 63659.4 \\
\hline 108300 & 77.9485 & 153.7635 & 2279.17 & 821 & 1 & 0.0 \\
\hline 108450 & 77.9487 & 153.7572 & 2279.61 & 822 & 1 & 0.0 \\
\hline 108600 & 77.9489 & 153.7508 & 2280.11 & 823 & 1 & 63644.3 \\
\hline 108750 & 77.9490 & 153.7444 & 2279.90 & 824 & 1 & 0.0 \\
\hline 108900 & 77.9492 & 153.7380 & 2280.08 & 825 & 1 & 0.0 \\
\hline 109050 & 77.9494 & 153.7316 & 2280.31 & 826 & 1 & 63589.6 \\
\hline 109200 & 77.9495 & 153.7253 & 2280.69 & 827 & 1 & 0.0 \\
\hline
\end{tabular}




\begin{tabular}{|c|c|c|c|c|c|c|}
\hline Distance $(\mathbf{m})$ & $\begin{array}{l}\text { Flag Loc. } \\
\left.\text { Latitude ( }{ }^{\circ} \mathbf{S}\right)\end{array}$ & $\begin{array}{c}\text { Flag Loc. } \\
\text { Longitude }\left({ }^{\circ} \mathrm{E}\right)\end{array}$ & Elevation (m) & $\begin{array}{c}\text { Shot } \\
\text { Number }\end{array}$ & $\begin{array}{l}\text { Julian } \\
\text { Day }\end{array}$ & $\begin{array}{l}\text { Total Mag. } \\
\text { Field (nT) }\end{array}$ \\
\hline 709350 & 77.9497 & 153.7189 & $\overline{2280.60}$ & $\overline{828}$ & 1 & $\overline{0.0}$ \\
\hline 109500 & 77.9499 & 153.7125 & 2280.83 & 829 & 1 & 63530.6 \\
\hline 109650 & 77.9500 & 153.7061 & 2281.11 & 830 & 1 & 0.0 \\
\hline 109800 & 77.9502 & 153.6997 & 2281.09 & 831 & 1 & 0.0 \\
\hline 109950 & 77.9504 & 153.6933 & 2281.08 & 832 & 1 & 63570.9 \\
\hline 110100 & 77.9505 & 153.6869 & 2281.26 & 833 & 1 & 0.0 \\
\hline 110250 & 77.9507 & 153.6806 & 2281.23 & 834 & 1 & 0.0 \\
\hline 110400 & 77.9509 & 153.6742 & 2281.20 & 835 & 1 & 63590.6 \\
\hline 110550 & 77.9510 & 153.6678 & 2281.09 & 836 & 1 & 0.0 \\
\hline 110700 & 77.9512 & 153.6614 & 2281.21 & 837 & 1 & 0.0 \\
\hline 110850 & 77.9514 & 153.6550 & 2281.12 & 838 & 1 & 63571.0 \\
\hline 111000 & 77.9515 & 153.6487 & 2281.39 & 839 & 1 & 0.0 \\
\hline 111150 & 77.9517 & 153.6423 & 2281.37 & 840 & 1 & 0.0 \\
\hline 111300 & 77.9519 & 153.6359 & 2281.37 & 841 & 1 & 63576.5 \\
\hline 111450 & 77.9520 & 153.6295 & 2281.31 & 842 & 1 & 0.0 \\
\hline 111600 & 77.9522 & 153.6231 & 2281.60 & 843 & 1 & 63599.1 \\
\hline 111750 & 77.9524 & 153.6167 & 2281.87 & 844 & 1 & 63567.0 \\
\hline 111900 & 77.9525 & 153.6103 & 2282.05 & 845 & 1 & 0.0 \\
\hline 112050 & 77.9527 & 153.6039 & 2282.20 & 846 & 1 & 0.0 \\
\hline 112200 & 77.9529 & 153.5975 & 2282.36 & 847 & 1 & 63582.7 \\
\hline 112350 & 77.9530 & 153.5912 & 2282.74 & 848 & 1 & 0.0 \\
\hline 112500 & 77.9532 & 153.5848 & 2282.83 & 849 & 1 & 0.0 \\
\hline 112650 & 77.9534 & 153.5784 & 2283.05 & 850 & 1 & 63553.2 \\
\hline 112800 & 77.9535 & 153.5720 & 2283.28 & 851 & 1 & 0.0 \\
\hline 112950 & 77.9537 & 153.5656 & 2283.46 & 852 & 1 & 0.0 \\
\hline 113100 & 77.9539 & 153.5592 & 2283.76 & 853 & 1 & 63568.3 \\
\hline 113250 & 77.9540 & 153.5529 & 2284.23 & 854 & 1 & 0.0 \\
\hline 113400 & 77.9542 & 153.5465 & 2284.61 & 855 & 1 & 0.0 \\
\hline 113550 & 77.9544 & 153.5401 & 2284.28 & 856 & 1 & 63537.5 \\
\hline 113700 & 77.9545 & 153.5337 & 2284.81 & 857 & 1 & 63522.1 \\
\hline 113850 & 77.9547 & 153.5273 & 2285.20 & 858 & 1 & 0.0 \\
\hline 114000 & 77.9549 & 153.5209 & 2285.55 & 859 & 1 & 63530.6 \\
\hline 114150 & 77.9550 & 153.5145 & 2285.96 & 860 & 1 & 0.0 \\
\hline 114300 & 77.9552 & 153.5081 & 2286.21 & 861 & 1 & 0.0 \\
\hline 114450 & 77.9554 & 153.5017 & 2286.56 & 862 & 1 & 63536.3 \\
\hline 114600 & 77.9555 & 153.4954 & 2286.76 & 863 & 1 & 0.0 \\
\hline 114750 & 77.9557 & 153.4890 & 2286.80 & 864 & 1 & 0.0 \\
\hline 114900 & 77.9559 & 153.4826 & 2287.24 & 865 & 1 & 63533.4 \\
\hline 115050 & 77.9560 & 153.4762 & 2287.18 & 866 & 1 & 0.0 \\
\hline 115200 & 77.9562 & 153.4698 & 2287.46 & 867 & 1 & 0.0 \\
\hline 115350 & 77.9564 & 153.4634 & 2287.06 & 868 & 1 & 63528.6 \\
\hline 115500 & 77.9565 & 153.4570 & 2287.71 & 869 & 1 & 0.0 \\
\hline 115650 & 77.9567 & 153.4507 & 2287.63 & 870 & 1 & 0.0 \\
\hline 115800 & 77.9569 & 153.4442 & 2288.14 & 871 & 1 & 63546.0 \\
\hline 115950 & 77.9570 & 153.4379 & 2288.32 & 872 & 1 & 0.0 \\
\hline 116100 & 77.9572 & 153.4315 & 2288.33 & 873 & 1 & 0.0 \\
\hline 116250 & 77.9574 & 153.4251 & 2288.50 & 874 & 1 & 63551.6 \\
\hline 116400 & 77.9575 & 153.4187 & 2288.49 & 875 & 1 & 0.0 \\
\hline 116550 & 77.9577 & 153.4123 & 2288.44 & 876 & 1 & 0.0 \\
\hline 116700 & 77.9579 & 153.4059 & 2288.10 & 877 & 1 & 63555.3 \\
\hline 116850 & 77.9580 & 153.3995 & 2287.80 & 878 & 1 & 0.0 \\
\hline 117000 & 77.9582 & 153.3932 & 2287.69 & 879 & 1 & 0.0 \\
\hline
\end{tabular}




\begin{tabular}{|c|c|c|c|c|c|c|}
\hline Distance $(\mathrm{m})$ & $\begin{array}{c}\text { Flag Loc. } \\
\text { Latitude ( }{ }^{\circ} \text { S) }\end{array}$ & $\begin{array}{c}\text { Flag Loc. } \\
\text { Longitude }\left({ }^{\circ} \mathrm{E}\right)\end{array}$ & Elevation (m) & $\begin{array}{c}\text { Shot } \\
\text { Number }\end{array}$ & $\begin{array}{c}\text { Julian } \\
\text { Day }\end{array}$ & $\begin{array}{l}\text { Total Mag. } \\
\text { Field (nT) }\end{array}$ \\
\hline 17750 & 77.9584 & $7 5 \longdiv { 3 . 3 8 6 8 }$ & 2287.45 & 880 & 71 & 63556.9 \\
\hline 117300 & 77.9585 & 153.3804 & 2287.33 & 881 & 1 & 0.0 \\
\hline 117450 & 77.9587 & 153.3740 & 2287.02 & 882 & 1 & 0.0 \\
\hline 117600 & 77.9589 & 153.3676 & 2286.81 & 883 & 1 & 63561.2 \\
\hline 117750 & 77.9590 & 153.3612 & 2287.04 & 884 & 1 & 0.0 \\
\hline 117900 & 77.9592 & 153.3548 & 2286.91 & 885 & 1 & 63574.5 \\
\hline 118050 & 77.9594 & 153.3484 & 2286.81 & 886 & 1 & 63560.3 \\
\hline 118200 & 77.9595 & 153.3420 & 2287.12 & 887 & 1 & 0.0 \\
\hline 118350 & 77.9597 & 153.3356 & 2287.46 & 888 & 1 & 0.0 \\
\hline 118500 & 77.9599 & 153.3293 & 2287.65 & 889 & 1 & 63557.1 \\
\hline 118650 & 77.9600 & 153.3229 & 2287.98 & 890 & 1 & 0.0 \\
\hline 118800 & 77.9602 & 153.3165 & 2288.38 & 891 & 1 & 0.0 \\
\hline 118950 & 77.9604 & 153.3101 & 2288.80 & 892 & 1 & 63570.5 \\
\hline 119100 & 77.9605 & 153.3037 & 2289.30 & 893 & 1 & 0.0 \\
\hline 119250 & 77.9607 & 153.2973 & 2289.76 & 894 & 1 & 0.0 \\
\hline 119400 & 77.9609 & 153.2909 & 2290.31 & 895 & 1 & 63558.5 \\
\hline 119550 & 77.9610 & 153.2845 & 2290.69 & 896 & 1 & 0.0 \\
\hline 119700 & 77.9612 & 153.2781 & 2291.26 & 897 & 1 & 0.0 \\
\hline 119850 & 77.9613 & 153.2718 & 2291.74 & 898 & 1 & 63554.4 \\
\hline 120000 & 77.9615 & 153.2653 & 2292.30 & 899 & 1 & 63550.2 \\
\hline 120150 & 77.9617 & 153.2589 & 2292.67 & 900 & 1 & 0.0 \\
\hline 120300 & 77.9619 & 153.2526 & 2293.39 & 901 & 1 & 63548.5 \\
\hline 120450 & 77.9620 & 153.2462 & 2294.40 & 902 & 1 & 0.0 \\
\hline 120600 & 77.9622 & 153.2398 & 2294.59 & 903 & 1 & 0.0 \\
\hline 120750 & 77.9624 & 153.2334 & 2295.29 & 904 & 2 & 63564.6 \\
\hline 120900 & 77.9625 & 153.2270 & 2295.72 & 905 & 2 & 0.0 \\
\hline 121050 & 77.9627 & 153.2206 & 2296.23 & 906 & 2 & 0.0 \\
\hline 121200 & 77.9629 & 153.2142 & 2297.06 & 907 & 2 & 63574.8 \\
\hline 121350 & 77.9630 & 153.2078 & 2297.31 & 908 & 2 & 0.0 \\
\hline 121500 & 77.9632 & 153.2014 & 2297.76 & 909 & 2 & 0.0 \\
\hline 121650 & 77.9633 & 153.1951 & 2298.20 & 910 & 2 & 63550.3 \\
\hline 121800 & 77.9635 & 153.1887 & 2298.49 & 911 & 2 & 0.0 \\
\hline 121950 & 77.9637 & 153.1823 & 2298.86 & 912 & 2 & 0.0 \\
\hline 122100 & 77.9639 & 153.1759 & 2298.73 & 913 & 2 & 63551.8 \\
\hline 122250 & 77.9640 & 153.1694 & 2298.67 & 914 & 2 & 0.0 \\
\hline 122400 & 77.9641 & 153.1631 & 2298.90 & 915 & 2 & 0.0 \\
\hline 122550 & 77.9643 & 153.1566 & 2298.76 & 916 & 2 & 63550.6 \\
\hline 122700 & 77.9644 & 153.1503 & 2299.14 & 917 & 2 & 0.0 \\
\hline 122850 & 77.9646 & 153.1438 & 2298.83 & 918 & 2 & 0.0 \\
\hline 123000 & 77.9647 & 153.1375 & 2298.95 & 919 & 2 & 63555.3 \\
\hline 123150 & 77.9649 & 153.1310 & 2298.85 & 920 & 2 & 0.0 \\
\hline 123300 & 77.9650 & 153.1246 & 2298.75 & 921 & 2 & 0.0 \\
\hline 123450 & 77.9652 & 153.1182 & 2298.66 & 922 & 2 & 63551.2 \\
\hline 123600 & 77.9653 & 153.1118 & 2298.41 & 923 & 2 & 0.0 \\
\hline 123750 & 77.9655 & 153.1054 & 2298.42 & 924 & 2 & 0.0 \\
\hline 123900 & 77.9656 & 153.0990 & 2298.28 & 925 & 2 & 63541.4 \\
\hline 124050 & 77.9658 & 153.0926 & 2297.84 & 926 & 2 & 0.0 \\
\hline 124200 & 77.9659 & 153.0862 & 2296.66 & 927 & 2 & 63529.7 \\
\hline 124350 & 77.9661 & 153.0798 & 2295.72 & 928 & 2 & 63530.4 \\
\hline 124500 & 77.9662 & 153.0734 & 2295.03 & 929 & 2 & 0.0 \\
\hline 124650 & 77.9664 & 153.0670 & 2293.95 & 930 & 2 & 0.0 \\
\hline 124800 & 77.9665 & 153.0606 & 2293.22 & 931 & 2 & 63539.0 \\
\hline
\end{tabular}




\begin{tabular}{|c|c|c|c|c|c|c|}
\hline Distance (m) & $\begin{array}{l}\text { Flag Loc. } \\
\left.\text { Latitude ( }{ }^{\circ} \mathrm{S}\right)\end{array}$ & $\begin{array}{c}\text { Flag Loc. } \\
\text { Longitude }\left({ }^{\circ} \mathrm{E}\right)\end{array}$ & Elevation $(\mathbf{m})$ & $\begin{array}{c}\text { Shot } \\
\text { Number }\end{array}$ & $\begin{array}{c}\text { Julian } \\
\text { Day }\end{array}$ & $\begin{array}{l}\text { Total Mag. } \\
\text { Field (nT) }\end{array}$ \\
\hline 124950 & 77.9666 & 153.0542 & 2292.60 & 932 & $\overline{2}$ & 0.0 \\
\hline 125100 & 77.9668 & 153.0478 & 2292.49 & 933 & 2 & 0.0 \\
\hline 125250 & 77.9669 & 153.0414 & 2292.30 & 934 & 2 & 63556.1 \\
\hline 125400 & 77.9671 & 153.0350 & 2292.39 & 935 & 2 & 0.0 \\
\hline 125550 & 77.9672 & 153.0286 & 2293.16 & 936 & 2 & 0.0 \\
\hline 125700 & 77.9674 & 153.0222 & 2294.31 & 937 & 2 & 63570.6 \\
\hline 125850 & 77.9675 & 153.0158 & 2296.15 & 938 & 2 & 0.0 \\
\hline 126000 & 77.9677 & 153.0094 & 2297.47 & 939 & 2 & 0.0 \\
\hline 126150 & 77.9678 & 153.0030 & 2299.72 & 940 & 2 & 63577.6 \\
\hline 126300 & 77.9680 & 152.9966 & 2301.83 & 941 & 2 & 63580.4 \\
\hline 126450 & 77.9681 & 152.9902 & 2303.88 & 942 & 2 & 0.0 \\
\hline 126600 & 77.9682 & 152.9838 & 2305.73 & 943 & 2 & 63587.6 \\
\hline 126750 & 77.9684 & 152.9774 & 2307.92 & 944 & 2 & 0.0 \\
\hline 126900 & 77.9685 & 152.9710 & 2309.72 & 945 & 2 & 0.0 \\
\hline 127050 & 77.9687 & 152.9646 & 2311.44 & 946 & 2 & 63593.1 \\
\hline 127200 & 77.9688 & 152.9581 & 2313.15 & 947 & 2 & 0.0 \\
\hline 127350 & 77.9690 & 152.9518 & 2314.81 & 948 & 2 & 0.0 \\
\hline 127500 & 77.9691 & 152.9453 & 2316.51 & 949 & 2 & 63593.4 \\
\hline 127650 & 77.9693 & 152.9389 & 2317.44 & 950 & 2 & 0.0 \\
\hline 127800 & 77.9694 & 152.9325 & 2318.91 & 951 & 2 & 0.0 \\
\hline 127950 & 77.9696 & 152.9260 & 2319.96 & 952 & 3 & 63583.6 \\
\hline 128100 & 77.9697 & 152.9196 & 2320.69 & 953 & 3 & 0.0 \\
\hline 128250 & 77.9699 & 152.9132 & 2321.75 & 954 & 3 & 0.0 \\
\hline 128400 & 77.9700 & 152.9068 & 2322.62 & 955 & 3 & 63595.9 \\
\hline 128550 & 77.9701 & 152.9004 & 2323.09 & 956 & 3 & 0.0 \\
\hline 128700 & 77.9703 & 152.8940 & 2324.18 & 957 & 3 & 0.0 \\
\hline 128850 & 77.9704 & 152.8876 & 2324.69 & 958 & 3 & 63597.2 \\
\hline 129000 & 77.9706 & 152.8812 & 2325.34 & 959 & 3 & 0.0 \\
\hline 129150 & 77.9707 & 152.8748 & 2326.16 & 960 & 3 & 0.0 \\
\hline 129300 & 77.9709 & 152.8684 & 2326.45 & 961 & 3 & 63605.6 \\
\hline 129450 & 77.9710 & 152.8620 & 2326.89 & 962 & 3 & 0.0 \\
\hline 129600 & 77.9712 & 152.8556 & 2327.28 & 963 & 3 & 0.0 \\
\hline 129750 & 77.9713 & 152.8492 & 2327.91 & 964 & 3 & 63573.1 \\
\hline 129900 & 77.9715 & 152.8427 & 2328.43 & 965 & 3 & 0.0 \\
\hline 130050 & 77.9716 & 152.8363 & 2328.98 & 966 & 3 & 0.0 \\
\hline 130200 & 77.9717 & 152.8299 & 2329.52 & 967 & 3 & 63564.0 \\
\hline 130350 & 77.9719 & 152.8235 & 2329.82 & 968 & 3 & 0.0 \\
\hline 130500 & 77.9720 & 152.8171 & 2330.17 & 969 & 3 & 0.0 \\
\hline 130650 & 77.9722 & 152.8107 & 2330.52 & 970 & 3 & 63531.0 \\
\hline 130800 & 77.9723 & 152.8043 & 2330.89 & 971 & 3 & 0.0 \\
\hline 130950 & 77.9725 & 152.7979 & 2331.14 & 972 & 3 & 0.0 \\
\hline 131100 & 77.9726 & 152.7915 & 2331.54 & 973| & 3 & 63523.9 \\
\hline 131250 & 77.9728 & 152.7851 & 2331.85 & 974 & 3 & 0.0 \\
\hline 131400 & 77.9729 & 152.7787 & 2332.45 & 975 & 3. & 0.0 \\
\hline 131550 & 77.9730 & 152.7723 & 2332.40 & 976| & 3) & 63549.2 \\
\hline 131700 & 77.9732 & 152.7659 & 2332.84 & 977| & 3. & 0.0 \\
\hline 131850 & 77.9733 & 152.7594 & 2333.23 & 978 & 3) & 63543.6 \\
\hline 132000 & 77.9735 & 152.7530 & 2333.59 & 979 & 3) & 63528.4 \\
\hline 132150 & 77.9736 & 152.7466 & 2333.89 & 980 & 3 & 0.0 \\
\hline 132300 & 77.9738 & 152.7402 & 2334.30 & 981 & 3 & 0.0 \\
\hline 132450 & 77.9739 & 152.7338 & 2334.30 & 982 & 3 & 63486.0 \\
\hline 132600 & 77.9741 & 152.7274 & 2334.44 & 983 & 3 & 0.0 \\
\hline
\end{tabular}




\begin{tabular}{|c|c|c|c|c|c|c|}
\hline Distance $(\mathrm{m})$ & $\begin{array}{l}\text { Flag Loc. } \\
\left.\text { Latitude ( }{ }^{\circ} \mathbf{S}\right)\end{array}$ & $\begin{array}{c}\text { Flag Loc. } \\
\text { Longitude }\left({ }^{\circ} E\right)\end{array}$ & Elevation (m) & $\begin{array}{c}\text { Shot } \\
\text { Number }\end{array}$ & $\begin{array}{c}\text { Julian } \\
\text { Day }\end{array}$ & $\begin{array}{l}\text { Total Mag. } \\
\text { Field (nT) }\end{array}$ \\
\hline 732750 & 77.9742 & 752.7210 & 2334.54 & 984 & 3 & $\overline{0.0}$ \\
\hline 132900 & 77.9743 & 152.7146 & 2334.60 & 985 & 3 & 63466.0 \\
\hline 133050 & 77.9745 & 152.7082 & 2334.73 & 986 & 3 & 0.0 \\
\hline 133200 & 77.9746 & 152.7018 & 2334.82 & 987 & 3 & 0.0 \\
\hline 133350 & 77.9748 & 152.6954 & 2334.64 & 988 & 3 & 63446.3 \\
\hline 133500 & 77.9749 & 152.6889 & 2334.60 & 989 & 3 & 0.0 \\
\hline 133650 & 77.9751 & 152.6825 & 2334.48 & 990 & 3 & 0.0 \\
\hline 133800 & 77.9752 & 152.6761 & 2334.51 & 991 & 3 & 63435.9 \\
\hline 133950 & 77.9754 & 152.6697 & 2334.32 & 992 & 3 & 63429.0 \\
\hline 134100 & 77.9755 & 152.6633 & 2334.06 & 993 & 3 & 0.0 \\
\hline 134250 & 77.9756 & 152.6569 & 2333.85 & 994 & 3 & 63438.5 \\
\hline 134400 & 77.9758 & 152.6505 & 2333.54 & 995 & 3 & 0.0 \\
\hline 134550 & 77.9759 & 152.6441 & 2333.30 & 996 & 3 & 0.0 \\
\hline 134700 & 77.9761 & 152.6376 & 2332.92 & 997 & 3 & 63432.3 \\
\hline 134850 & 77.9762 & 152.6312 & 2332.61 & 998 & 3 & 0.0 \\
\hline 135000 & 77.9764 & 152.6248 & 2332.18 & 999 & 3 & 0.0 \\
\hline 135150 & 77.9765 & 152.6184 & 2331.90 & 1000 & 3 & 63427.7 \\
\hline 135300 & 77.9767 & 152.6120 & 2331.51 & 1001 & 3 & 0.0 \\
\hline 135450 & 77.9768 & 152.6056 & 2330.88 & 1002 & 3 & 0.0 \\
\hline 135600 & 77.9769 & 152.5992 & 2330.73 & 1003 & 3 & 63450.0 \\
\hline 135750 & 77.9771 & 152.5928 & 2330.32 & 1004 & 3 & 0.0 \\
\hline 135900 & 77.9772 & 152.5864 & 2330.11 & 1005 & 3 & 0.0 \\
\hline 136050 & 77.9774 & 152.5799 & 2329.96 & 1006 & 3 & 63439.4 \\
\hline 136200 & 77.9775 & 152.5735 & 2329.41 & 1007 & 3 & 0.0 \\
\hline 136350 & 77.9777 & 152.5671 & 2329.08 & 1008 & 3 & 0.0 \\
\hline 136500 & 77.9778 & 152.5607 & 2328.84 & 1009 & 3 & 63486.2 \\
\hline 136650 & 77.9780 & 152.5543 & 2328.51 & 1010 & 3 & 0.0 \\
\hline 136800 & 77.9781 & 152.5479 & 2327.81 & 1011 & 3 & 0.0 \\
\hline 136950 & 77.9782 & 152.5415 & 2327.60 & 1012 & 3 & 63481.5 \\
\hline 137100 & 77.9784 & 152.5351 & 2327.28 & 1013 & 3 & 0.0 \\
\hline 137250 & 77.9785 & 152.5287 & 2326.94 & 1014 & 3 & 0.0 \\
\hline 137400 & 77.9787 & 152.5223 & 2326.54 & 1015 & 3 & 63477.6 \\
\hline 137550 & 77.9788 & 152.5158 & 2326.45 & 1016 & 3 & 0.0 \\
\hline 137700 & 77.9790 & 152.5094 & 2325.77 & 1017 & 3 & 0.0 \\
\hline 137850 & 77.9791 & 152.5030 & 2325.52 & 1018 & 3 & 63485.6 \\
\hline 138000 & 77.9792 & 152.4966 & 2325.08 & 1019 & 3 & 0.0 \\
\hline 138150 & 77.9794 & 152.4902 & 2324.86 & 1020 & 3 & 63487.5 \\
\hline 138300 & 77.9795 & 152.4837 & 2324.34 & 1021 & 3 & 63491.3 \\
\hline 138450 & 77.9797 & 152.4773 & 2324.08 & 1022 & 3 & 0.0 \\
\hline 138600 & 77.9798 & 152.4709 & 2323.71 & 1023 & 3 & 0.0 \\
\hline 138750 & 77.9799 & 152.4645 & 2323.41 & 1024 & 3 & 63499.5 \\
\hline 138900 & 77.9800 & 152.4581 & 2323.26 & 1025 & 3 & 0.0 \\
\hline 139050 & 77.9802 & 152.4517 & 2322.92 & 1026 & 3 & 0.0 \\
\hline 139200 & 77.9803 & 152.4453 & 2322.83 & 1027 & 3 & 63516.1 \\
\hline 139350 & 77.9804 & 152.4388 & 2322.31 & 1028 & 3 & 0.0 \\
\hline 139500 & 77.9805 & 152.4324 & 2322.12 & 1029 & 3 & 0.0 \\
\hline 139650 & 77.9807 & 152.4260 & 2321.84 & 1030 & 3) & 63533.4 \\
\hline 139800 & 77.9808 & 152.4196 & 2321.65 & 1031 & 3 & 0.0 \\
\hline 139950 & 77.9809 & 152.4131 & 2321.53 & 1032 & 3 & 0.0 \\
\hline 140100 & 77.9810 & 152.4067 & 2321.55 & 1033 & 3 & 63544.8 \\
\hline 140250 & 77.9812 & 152.4003 & 2321.19 & 1034 & 3 & 63541.1 \\
\hline 140400 & 77.9813 & 152.3939 & 2320.81 & 1035 & 3 & 0.0 \\
\hline
\end{tabular}




\begin{tabular}{|c|c|c|c|c|c|c|}
\hline Distance $(\mathrm{m})$ & $\begin{array}{l}\text { Flag Loc. } \\
\text { Latitude }\left({ }^{\circ} \mathrm{S}\right)\end{array}$ & $\begin{array}{c}\text { Flag Loc. } \\
\text { Longitude }\left({ }^{\circ} \mathrm{E}\right)\end{array}$ & Elevation (m) & $\begin{array}{l}\text { Shot } \\
\text { Number }\end{array}$ & $\begin{array}{c}\text { Julian } \\
\text { Day }\end{array}$ & $\begin{array}{l}\text { Total Mag. } \\
\text { Field (nT) }\end{array}$ \\
\hline 740550 & 77.9814 & 152.3874 & 2320.73 & 1036 & 3 & 63538.6 \\
\hline 140700 & 77.9816 & 152.3810 & 2320.54 & 1037 & 3 & 0.0 \\
\hline 140850 & 77.9817 & 152.3746 & 2320.54 & 1038 & 3 & 0.0 \\
\hline 141000 & 77.9818 & 152.3682 & 2320.14 & 1039 & 3 & 63553.1 \\
\hline 141150 & 77.9819 & 152.3618 & 2320.03 & 1040 & 3 & 0.0 \\
\hline 141300 & 77.9821 & 152.3553 & 2319.97| & 1041 & 3 & 0.0 \\
\hline 141450 & 77.9822 & 152.3489 & 2319.99 & 1042 & 3 & 63567.5 \\
\hline 141600 & 77.9823 & 152.3425 & 2319.68 & 1043 & 3 & 0.0 \\
\hline 141750 & 77.9825 & 152.3361 & 2319.71 & 1044 & 3 & 0.0 \\
\hline 141900 & 77.9826 & 152.3297 & 2319.73 & 1045 & 3 & 63585.4 \\
\hline 142050 & 77.9827 & 152.3232 & 2319.59 & 1046 & 3 & 0.0 \\
\hline 142200 & 77.9828 & 152.3168 & 2319.50 & 1047 & 3 & 0.0 \\
\hline 142350 & 77.9830 & 152.3104 & 2319.63 & 1048 & 3 & 63586.8 \\
\hline 142500 & 77.9831 & 152.3040 & 2319.54 & 1049 & 3 & 0.0 \\
\hline 142650 & 77.9832 & 152.2975 & 2319.90 & 1050 & 3 & 0.0 \\
\hline 142800 & 77.9833 & 152.2911 & 2319.61 & 1051 & 3 & 63585.5 \\
\hline 142950 & 77.9835 & 152.2847 & 2319.61 & 1052 & 3 & 0.0 \\
\hline 143100 & 77.9836 & 152.2783 & 2319.91 & 1053 & 3 & 0.0 \\
\hline 143250 & 77.9837 & 152.2719 & 2319.81 & 1054 & 3 & 63588.9 \\
\hline 143400 & 77.9838 & 152.2654 & 2319.70 & 1055 & 3 & 0.0 \\
\hline 143550 & 77.9840 & 152.2590 & 2319.72 & 1056 & 3 & 0.0 \\
\hline 143700 & 77.9841 & 152.2526 & 2319.61 & 1057 & 3 & 63593.8 \\
\hline 143850 & 77.9842 & 152.2461 & 2319.69 & 1058 & 3 & 63596.5 \\
\hline 144000 & 77.9844 & 152.2397 & 2319.77 & 1059 & 3 & 0.0 \\
\hline 144150 & 77.9845 & 152.2333 & 2320.09 & 1060 & 4 & 63513.9 \\
\hline 144300 & 77.9846 & 152.2269 & 2320.16 & 1061 & 4 & 0.0 \\
\hline 144450 & 77.9847 & 152.2205 & 2320.24 & 1062 & 4 & 0.0 \\
\hline 144600 & 77.9849 & 152.2140 & 2320.53 & 1063 & 4 & 63502.9 \\
\hline 144750 & 77.9850 & 152.2076 & 2320.77 & 1064 & 4 & 0.0 \\
\hline 144900 & 77.9851 & 152.2012 & 2320.93 & 1065 & 4 & 0.0 \\
\hline 145050 & 77.9852 & 152.1948 & 2321.20 & 1066 & 4 & 63514.0 \\
\hline 145200 & 77.9854 & 152.1884 & 2321.52 & 1067 & 4 & 0.0 \\
\hline 145350 & 77.9855 & 152.1819 & 2321.53 & 1068 & 4 & 0.0 \\
\hline 145500 & 77.9856 & 152.1755 & 2321.68 & 1069 & 4 & 63519.3 \\
\hline 145650 & 77.9857 & 152.1691 & 2321.98 & 1070 & 4 & 0.0 \\
\hline 145950 & 77.9860 & 152.1562 & 2322.62 & 1072 & 4 & 63529.9 \\
\hline 146100 & 77.9861 & 152.1498 & 2323.08 & 1073 & 4 & 0.0 \\
\hline 146250 & 77.9863 & 152.1434 & 2323.07 & 1074 & 4 & 0.0 \\
\hline 146400 & 77.9864 & 152.1369 & 2323.13 & 1075 & 4 & 63566.0 \\
\hline 146550 & 77.9865 & 152.1305 & 2323.66 & 1076 & 4 & 0.0 \\
\hline 146700 & 77.9866 & 152.1241 & 2323.60 & 1077 & 4 & 0.0 \\
\hline 146850 & 77.9868 & 152.1177 & 2323.91 & 1078 & 4 & 63569.4 \\
\hline 147000 & 77.9869 & 152.1113 & 2324.07 & 1079 & 4 & 0.0 \\
\hline 147150 & 77.9870 & 152.1048 & 2324.03 & 1080 & 4 & 0.0 \\
\hline 147300 & 77.9871 & 152.0984 & 2324.31 & 1081 & 4 & 63580.6 \\
\hline 147450 & 77.9873 & 152.0920 & 2324.23 & 1082 & 4 & 0.0 \\
\hline 147600 & 77.9874 & 152.0856 & 2324.22 & 1083 & 4 & 0.0 \\
\hline 147750 & 77.9875 & 152.0791 & 2324.21 & wo1084 & 4 & 63585.5 \\
\hline 147900 & 77.9877 & 152.0727 & 2324.04 & wo1085 & 4 & 63597.8 \\
\hline 148050 & 77.9878 & 152.0663 & 2324.33 & wo1086 & 4 & 0.0 \\
\hline 148200 & 77.9879 & 152.0598 & 2324.08 & & 4 & 0.0 \\
\hline 148350 & 77.9880 & 152.0534 & 2324.43 & wo1088 & 4 & 63595.7 \\
\hline
\end{tabular}




\begin{tabular}{|c|c|c|c|c|c|c|}
\hline Distance $(m)$ & $\begin{array}{l}\text { Flag Loc. } \\
\text { Latitude ( }{ }^{\circ} \text { S) }\end{array}$ & $\begin{array}{c}\text { Flag Loc. } \\
\text { Longitude }\left({ }^{\circ} \mathrm{E}\right)\end{array}$ & Elevation (m) & $\begin{array}{c}\text { Shot } \\
\text { Number }\end{array}$ & $\begin{array}{c}\text { Julian } \\
\text { Day }\end{array}$ & $\begin{array}{l}\text { Total Mag. } \\
\text { Fiold (nT) }\end{array}$ \\
\hline 148500 & 77.9882 & 152.0470 & 2324.40 & & 4 & 0.0 \\
\hline 148650 & 77.9883 & 152.0406 & 2324.44 & wo1090 & 4 & 0.0 \\
\hline 148800 & 77.9884 & 152.0341 & 2324.83 & & 4 & 0.0 \\
\hline 148950 & 77.9885 & 152.0277 & 2325.60 & wo1092 & 4 & 0.0 \\
\hline 149100 & 77.9887 & 152.0213 & 2326.05 & & 4 & 0.0 \\
\hline 149250 & 77.9888 & 152.0149 & 2326.59 & wo1094 & 4 & 63597.2 \\
\hline 149400 & 77.9889 & 152.0085 & 2327.17 & & 4 & 0.0 \\
\hline 149550 & 77.9890 & 152.0020 & 2327.75 & wo1096 & 4 & 0.0 \\
\hline 149700 & 77.9892 & 151.9956 & 2328.32 & & 4 & 0.0 \\
\hline 149850 & 77.9893 & 151.9892 & 2328.62 & wo1098 & 4 & 0.0 \\
\hline 150000 & 77.9894 & 151.9827 & 2329.58 & & 4 & 63584.6 \\
\hline 150150 & 77.9895 & 151.9763 & 2330.19 & wo1100 & 4 & 0.0 \\
\hline 150300 & 77.9897 & 151.9699 & 2330.58 & 1101 & 4 & 0.0 \\
\hline 150450 & 77.9898 & 151.9634 & 2330.84 & 1102 & 4 & 0.0 \\
\hline 150600 & 77.9899 & 151.9570 & 2331.26 & 1103 & 4 & 0.0 \\
\hline 150750 & 77.9900 & 151.9506 & 2331.68 & 1104 & 4 & 0.0 \\
\hline 150900 & 77.9902 & 151.9442 & 2332.04 & 1105 & 5 & 63580.8 \\
\hline 151050 & 77.9903 & 151.9377 & 2332.19 & 1106 & 5 & 0.0 \\
\hline 151200 & 77.9904 & 151.9313 & 2332.19 & 1107 & 5 & 0.0 \\
\hline 151350 & 77.9905 & 151.9249 & 2332.60 & 1108 & 5 & 63573.6 \\
\hline 151500 & 77.9907 & 151.9185 & 2332.98 & 1109 & 5 & 0.0 \\
\hline 151650 & 77.9908 & 151.9120 & 2333.02 & 1110 & 5 & 0.0 \\
\hline 151800 & 77.9909 & 151.9056 & 2333.29 & 1111 & 5 & 63566.4 \\
\hline 151950 & 77.9910 & 151.8992 & 2333.64 & 1112 & 5 & 0.0 \\
\hline 152100 & 77.9912 & 151.8927 & 2333.84 & 1113 & 5 & 63554.2 \\
\hline 152250 & 77.9913 & 151.8863 & 2333.99 & 1114 & 5 & 0.0 \\
\hline 152400 & 77.9914 & 151.8799 & 2334.02 & 1115 & 5 & 0.0 \\
\hline 152550 & 77.9915 & 151.8735 & 2334.25 & 1116 & 5 & 0.0 \\
\hline 152700 & 77.9917 & 151.8670 & 2334.37 & 1117 & 5 & 63566.3 \\
\hline 152850 & 77.9918 & 151.8606 & 2334.74 & 1118 & 5 & 0.0 \\
\hline 153000 & 77.9919 & 151.8542 & 2334.58 & 1119 & 5 & 0.0 \\
\hline 153150 & 77.9920 & 151.8477 & 2334.65 & 1120 & 5 & 63549.0 \\
\hline 153300 & 77.9922 & 151.8413 & 2334.64 & 1121 & 5 & 0.0 \\
\hline 153450 & 77.9923 & 151.8349 & 2335.12 & 1122 & 5 & 0.0 \\
\hline 153600 & 77.9924 & 151.8285 & 2334.91 & 1123 & 5 & 63543.3 \\
\hline 153750 & 77.9925 & 151.8221 & 2335.28 & 1124 & 5 & 0.0 \\
\hline 153900 & 77.9927 & 151.8156 & 2335.49 & 1125 & 5 & 0.0 \\
\hline 154050 & 77.9928 & 151.8092 & 2335.07 & 1126 & 5 & 63539.4 \\
\hline 154200 & 77.9929 & 151.8027 & 2335.32 & 1127 & 5 & 63540.1 \\
\hline 154350 & 77.9930 & 151.7963 & 2335.07 & 1128 & 5 & 0.0 \\
\hline 154500 & 77.9932 & 151.7899 & 2335.43 & 1129 & 5 & 63537.0 \\
\hline 154650 & 77.9933 & 151.7834 & 2335.29 & 1130 & 5 & 0.0 \\
\hline 154800 & 77.9934 & 151.7770 & 2335.13 & 1131 & 5 & 0.0 \\
\hline 154950 & 77.9935 & 151.7706 & 2335.14 & 1132 & 5 & 63519.9 \\
\hline 155100 & 77.9937 & 151.7642 & 2334.98 & 1133 & 5 & 0.0 \\
\hline 155250 & 77.9938 & 151.7578 & 2334.91 & 1134 & 5 & 0.0 \\
\hline 155400 & 77.9939 & 151.7513 & 2334.95 & 1135 & 5 & 63514.7 \\
\hline 155550 & 77.9940 & 151.7449 & 2334.98 & 1136 & 5 & 0.0 \\
\hline 155700 & 77.9942 & 151.7385 & 2335.12 & 1137 & 5 & 0.0 \\
\hline 155850 & 77.9943 & 151.7320 & 2335.40 & 1138 & 5 & 63510.4 \\
\hline 156000 & 77.9944 & 151.7256 & 2336.19 & 1139 & 5 & 0.0 \\
\hline 156150 & 77.9945 & 151.7192 & 2336.14 & 1140 & 5 & 0.0 \\
\hline
\end{tabular}




\begin{tabular}{|c|c|c|c|c|c|c|}
\hline Distance (m) & $\begin{array}{l}\text { Flag Loc. } \\
\text { Latitude ('S) }\end{array}$ & $\begin{array}{c}\text { Flag Loc. } \\
\text { Longitude }\left({ }^{\circ} \mathrm{E}\right)\end{array}$ & Elevation (m) & $\begin{array}{c}\text { Shot } \\
\text { Number }\end{array}$ & $\begin{array}{c}\text { Julian } \\
\text { Day }\end{array}$ & $\begin{array}{l}\text { Total Mag. } \\
\text { Field (nT) }\end{array}$ \\
\hline 756300 & 77.9947 & 151.7127 & 2337.80 & 1141 & 5 & 63514.3 \\
\hline 156450 & 77.9948 & 151.7063 & 2338.41 & 1142 & 5 & 0.0 \\
\hline 156600 & 77.9949 & 151.6999 & 2339.19 & 1143 & 5 & 0.0 \\
\hline 156750 & 77.9950 & 151.6934 & 2339.67 & 1144 & 5 & 63498.4 \\
\hline 156900 & 77.9951 & 151.6870 & 2340.51 & 1145 & 5 & 0.0 \\
\hline 157050 & 77.9953 & 151.6806 & 2341.47 & 1146 & 5 & 0.0 \\
\hline 157200 & 77.9954 & 151.6741 & 2341.92 & 1147 & 5 & 63503.8 \\
\hline 157350 & 77.9955 & 151.6677 & 2342.53 & 1148 & 5 & 0.0 \\
\hline 157500 & 77.9956 & 151.6613 & 2343.10 & 1149 & 5 & 0.0 \\
\hline 157650 & 77.9958 & 151.6549 & 2343.78 & 1150 & 5 & 63501.0 \\
\hline 157800 & 77.9959 & 151.6484 & 2344.14 & 1151 & 5 & 0.0 \\
\hline 157950 & 77.9960 & 151.6420 & 2344.61 & 1152 & 5 & 0.0 \\
\hline 158100 & 77.9961 & 151.6356 & 2344.51 & 1153 & 5 & 63494.4 \\
\hline 158250 & 77.9963 & 151.6291 & 2344.93 & 1154 & 5 & 63493.3 \\
\hline 158400 & 77.9964 & 151.6227 & 2344.72 & 1155 & 5 & 0.0 \\
\hline 158550 & 77.9965 & 151.6163 & 2344.61 & 1156 & 5 & 63480.7 \\
\hline 158700 & 77.9966 & 151.6098 & 2344.21 & 1157 & 5 & 0.0 \\
\hline 158850 & 77.9967 & 151.6034 & 2343.76 & 1158 & 5 & 0.0 \\
\hline 159000 & 77.9969 & 151.5970 & 2343.14 & 1159 & 5 & 63481.1 \\
\hline 159150 & 77.9970 & 151.5905 & 2342.64 & 1160 & 5 & 0.0 \\
\hline 159300 & 77.9971 & 151.5841 & 2341.90 & 1161 & 5 & 0.0 \\
\hline 159450 & 77.9972 & 151.5777 & 2340.98 & 1162 & 5 & 63480.2 \\
\hline 159600 & 77.9974 & 151.5713 & 2340.24 & 1163 & 5 & 0.0 \\
\hline 159750 & 77.9975 & 151.5648 & 2339.13 & 1164 & 5 & 0.0 \\
\hline 159900 & 77.9976 & 151.5584 & 2338.51 & 1165 & 5 & 63475.5 \\
\hline 160050 & 77.9977 & 151.5519 & 2338.58 & 1166 & 5 & 0.0 \\
\hline 160200 & 77.9979 & 151.5455 & 2336.90 & 1167 & 5 & 0.0 \\
\hline 160350 & 77.9980 & 151.5391 & 2336.13 & 1168 & 5 & 63478.8 \\
\hline 160500 & 77.9981 & 151.5327 & 2335.36 & 1169 & 5 & 0.0 \\
\hline 160650 & 77.9982 & 151.5262 & 2334.32 & 1170 & 5 & 0.0 \\
\hline 160800 & 77.9984 & 151.5198 & 2333.72 & 1171 & 5 & 63475.8 \\
\hline 160950 & 77.9985 & 151.5134 & 2333.02 & 1172 & 5 & 0.0 \\
\hline 161100 & 77.9986 & 151.5069 & 2332.35 & 1173 & 5 & 0.0 \\
\hline 161250 & 77.9987 & 151.5005 & 2332.06 & 1174 & 5 & 63478.8 \\
\hline 161400 & 77.9988 & 151.4941 & 2331.25 & 1175 & 5 & 0.0 \\
\hline 161550 & 77.9990 & 151.4876 & 2330.65 & 1176 & 5 & 0.0 \\
\hline 161700 & 77.9991 & 151.4812 & 2330.40 & 1177 & 5 & 63481.0 \\
\hline 161850 & 77.9992 & 151.4748 & 2330.03 & 1178 & 5 & 0.0 \\
\hline 162000 & 77.9993 & 151.4683 & 2329.79 & 1179 & 5 & 63484.6 \\
\hline 162150 & 77.9995 & 151.4619 & 2329.46 & 0 & 6 & 63447.6 \\
\hline 162300 & 77.9996 & 151.4555 & 2329.02 & 이 & 6 & 0.0 \\
\hline 162450 & 77.9997 & 151.4490 & 2329.04 & 이 & 6 & 0.0 \\
\hline 162600 & 77.9998 & 151.4426 & 2328.87 & o & 6 & 63484.4 \\
\hline 162750 & 77.9999 & 151.4362 & 2328.80 & 이 & 6 & 0.0 \\
\hline 162900 & 78.0001 & 151.4297 & 2328.53 & 이 & 6 & 0.0 \\
\hline 163050 & 78.0002 & 151.4233 & 2328.54 & ol & 6 & 63465.4 \\
\hline 163200 & 78.0003 & 151.4169 & 2328.45 & 0 & 6 & 0.0 \\
\hline 163350 & 78.0004 & 151.4104 & 2328.33 & 이 & 6 & 0.0 \\
\hline 163500 & 78.0006 & 151.4040 & 2328.46 & 0 & 6 & 63418.6 \\
\hline 163650 & 78.0007 & 151.3976 & 2328.34 & 0 & 6 & 0.0 \\
\hline 163800 & 78.0008 & 151.3911 & 2328.42 & o & 6 & 0.0 \\
\hline 163950 & 78.0009 & 151.3847 & 2328.70 & ol & 6 & 63424.6 \\
\hline
\end{tabular}




\begin{tabular}{|c|c|c|c|c|c|c|}
\hline Distance $(\mathbf{m})$ & $\begin{array}{l}\text { Flag Loc. } \\
\left.\text { Latitude ( }{ }^{\circ} \mathbf{S}\right)\end{array}$ & $\begin{array}{c}\text { Flag Loc. } \\
\text { Longitude }\left({ }^{\circ} \mathrm{E}\right)\end{array}$ & Elevation (m) & $\begin{array}{c}\text { Shot } \\
\text { Number }\end{array}$ & $\begin{array}{c}\text { Julian } \\
\text { Day }\end{array}$ & $\begin{array}{l}\text { Total Mag. } \\
\text { Field (nT) }\end{array}$ \\
\hline 164100 & 78.0071 & 151.3782 & $23 \overline{28.76}$ & 0 & $\overline{6}$ & 63428.2 \\
\hline 164250 & 78.0011 & 151.3718 & 2328.91 & 0 & 6 & 0.0 \\
\hline 164400 & 78.0012 & 151.3654 & 2329.28 & 0 & 6 & 63438.0 \\
\hline 164550 & 78.0013 & 151.3589 & 2329.21 & 0 & 6 & 0.0 \\
\hline 164700 & 78.0014 & 151.3525 & 2329.49 & 0 & 6 & 0.0 \\
\hline 164850 & 78.0015 & 151.3460 & 2329.68 & 0 & 6 & 63444.8 \\
\hline 165000 & 78.0016 & 151.3396 & 2329.94 & 0 & 6 & 0.0 \\
\hline 165150 & 78.0017 & 151.3331 & 2330.10 & 0 & 6 & 0.0 \\
\hline 165300 & 78.0018 & 151.3267 & 2330.48 & 0 & 6 & 63450.2 \\
\hline 165450 & 78.0019 & 151.3203 & 2330.35 & 0 & 6 & 0.0 \\
\hline 165600 & 78.0020 & 151.3138 & 2330.99 & 0 & 6 & 0.0 \\
\hline 165750 & 78.0021 & 151.3074 & 2331.35 & 0 & 6 & 63451.2 \\
\hline 165900 & 78.0022 & 151.3009 & 2331.57 & 0 & 6 & 0.0 \\
\hline 166050 & 78.0023 & 151.2945 & 2331.69 & 0 & 6 & 0.0 \\
\hline 166200 & 78.0024 & 151.2880 & 2332.35 & 0 & 6 & 63449.5 \\
\hline 166350 & 78.0025 & 151.2816 & 2332.60 & 0 & 6 & 0.0 \\
\hline 166500 & 78.0026 & 151.2751 & 2332.84 & 0 & 6 & 0.0 \\
\hline 166650 & 78.0027 & 151.2687 & 2333.62 & 0 & 6 & 63453.9 \\
\hline 166800 & 78.0027 & 151.2622 & 2333.69 & 0 & 6 & 0.0 \\
\hline 166950 & 78.0028 & 151.2558 & 2333.95 & 0 & 6 & 0.0 \\
\hline 167100 & 78.0029 & 151.2493 & 2334.34 & 0 & 6 & 63458.4 \\
\hline 167250 & 78.0030 & 151.2429 & 2334.71 & 0 & 6 & 0.0 \\
\hline 167400 & 78.0031 & 151.2365 & 2334.99 & 이 & 6 & 0.0 \\
\hline 167550 & 78.0032 & 151.2300 & 2335.60 & 0 & 6 & 63445.4 \\
\hline 167700 & 78.0033 & 151.2236 & 2336.02 & 0 & 6 & 0.0 \\
\hline 167850 & 78.0034 & 151.2171 & 2336.44 & 0 & 6 & 0.0 \\
\hline 168000 & 78.0035 & 151.2107 & 2336.94 & 0 & 6 & 63418.5 \\
\hline 168150 & 78.0036 & 151.2042 & 2337.34 & 0 & 6 & 0.0 \\
\hline 168300 & 78.0037 & 151.1977 & 2337.70 & 0 & 6 & 63418.9 \\
\hline 168450 & 78.0038 & 151.1913 & 2338.51 & 0 & 6 & 0.0 \\
\hline 168600 & 78.0039 & 151.1849 & 2338.64 & 0 & 6 & 0.0 \\
\hline 168750 & 78.0041 & 151.1785 & 2339.38 & 0 & 6 & 63426.6 \\
\hline 168900 & 78.0042 & 151.1720 & 2340.00 & 0 & 6 & 0.0 \\
\hline 16905 이 & 78.0043 & 151.1656 & 2340.27 & 0 & 6 & 0.0 \\
\hline 169200 & 78.0045 & 151.1592 & 2340.88 & 0 & 6 & 63427.0 \\
\hline 169350 & 78.0046 & 151.1528 & 2341.16 & 이 & 6 & 0.0 \\
\hline 169500 & 78.0048 & 151.1463 & 2341.45 & 0 & 6 & 0.0 \\
\hline 169650 & 78.0049 & 151.1399 & 2341.82 & 0 & 6 & 63423.0 \\
\hline 169800 & 78.0050 & 151.1335 & 2342.54 & 0 & 6 & 0.0 \\
\hline 169950 & 78.0051 & 151.1270 & 2342.66 & 0 & 6 & 0.0 \\
\hline 170100 & 78.0053 & 151.1206 & 2343.11 & 0 & 6 & 63437.0 \\
\hline 170250 & 78.0054 & 151.1142 & 2343.88 & 0 & 6 & 63435.3 \\
\hline 170400 & 78.0056 & 151.1077 & 2344.22 & 0 & 6 & 0.0 \\
\hline 170550 & 78.0057 & 151.1013 & 2344.62 & 0 & 6 & 0.0 \\
\hline 170700 & 78.0058 & 151.0949 & 2344.93 & o & 6 & 63425.2 \\
\hline 170850 & 78.0060 & 151.0884 & 2345.69 & 이 & 6 & 0.0 \\
\hline 171000 & 78.0071 & 151.0370 & 2348.31 & 0 & 6 & 0.0 \\
\hline 171150 & 78.0062 & 151.0756 & 2346.32 & 0 & 6 & 63422.5 \\
\hline 171300 & 78.0064 & 151.0692 & 2346.51 & 0 & 6 & 0.0 \\
\hline 171450 & 78.0065 & 151.0627 & 2346.96 & 0 & 6 & 0.0 \\
\hline 171600 & 78.0067 & 151.0563 & 2347.23 & 0 & 6 & 63420.0 \\
\hline 171750 & 78.0068 & 151.0499 & 2347.47 & 0 & 6 & 0.0 \\
\hline
\end{tabular}




\begin{tabular}{|c|c|c|c|c|c|c|}
\hline Distance $(\mathbf{m})$ & $\begin{array}{l}\text { Flag Loc. } \\
\text { Latitude ('S) }\end{array}$ & $\begin{array}{c}\text { Flag Loc. } \\
\text { Longitude }\left({ }^{\circ} \mathrm{E}\right)\end{array}$ & Elevation (m) & $\begin{array}{c}\text { Shot } \\
\text { Number }\end{array}$ & $\begin{array}{c}\text { Julian } \\
\text { Day }\end{array}$ & $\begin{array}{l}\text { Total Mag. } \\
\text { Field (nT) }\end{array}$ \\
\hline 171900 & 78.0069 & 157.0434 & 2347.85 & 이 & 6 & 0.0 \\
\hline 172200 & 78.0072 & 151.0306 & 2348.69 & 0 & 6 & 0.0 \\
\hline 172350 & 78.0074 & 151.0242 & 2349.13 & 0 & 6 & 0.0 \\
\hline 172500 & 78.0075 & 151.0177 & 2349.66 & 이 & 6 & 63418.4 \\
\hline 172650 & 78.0076 & 151.0113 & 2350.26 & 0 & 6 & 0.0 \\
\hline 172800 & 78.0078 & 151.0049 & 2350.71 & 이 & 6 & 0.0 \\
\hline 172950 & 78.0079 & 150.9984 & 2351.10 & 이 & 6 & 63426.1 \\
\hline 173100 & 78.0080 & 150.9920 & 2351.31 & 이 & 6 & 0.0 \\
\hline 173250 & 78.0082 & 150.9856 & 2351.59 & 이 & 6 & 0.0 \\
\hline 173400 & 78.0083 & 150.9792 & 2351.73 & 이 & 6 & 63426.4 \\
\hline 173550 & 78.0084 & 150.9727 & 2351.51 & 이 & 6 & 0.0 \\
\hline 173700 & 78.0086 & 150.9663 & 2351.41 & 이 & 6 & 63425.1 \\
\hline 173850 & 78.0087 & 150.9598 & 2351.53 & 이 & 6 & 0.0 \\
\hline 174000 & 78.0089 & 150.9534 & 2351.37 & 이 & 6 & 0.0 \\
\hline 174150 & 78.0090 & 150.9470 & 2351.05 & 이 & 6 & 63427.9 \\
\hline 174300 & 78.0091 & 150.9405 & 2350.89 & o & 6 & 0.0 \\
\hline 174450 & 78.0093 & 150.9341 & 2350.49 & 이 & 6 & 0.0 \\
\hline 174600 & 78.0094 & 150.9277 & 2347.71 & 이 & 6 & 63442.9 \\
\hline 174750 & 78.0095 & 150.9212 & 2349.97 & 이 & 6 & 0.0 \\
\hline 174900 & 78.0097 & 150.9148 & 2349.96 & 이 & 6 & 0.0 \\
\hline 175050 & 78.0098 & 150.9084 & 2350.01 & 이 & 6 & 63440.2 \\
\hline 175200 & 78.0099 & 150.9020 & 2349.80 & 이 & 6 & 0.0 \\
\hline 175350 & 78.0101 & 150.8955 & 2349.37 & 이 & 6 & 0.0 \\
\hline 175500 & 78.0102 & 150.8891 & 2349.41 & 이 & 6 & 63455.6 \\
\hline 175650 & 78.0103 & 150.8827 & 2349.12 & 이 & 6 & 0.0 \\
\hline 175800 & 78.0105 & 150.8762 & 2349.24 & 이 & 6 & 63460.0 \\
\hline 175950 & 78.0106 & 150.8698 & 2349.10 & ol & 6 & 0.0 \\
\hline 176100 & 78.0108 & 150.8633 & 2348.62 & 0 & 6 & 0.0 \\
\hline 176250 & 78.0109 & 150.8569 & 2348.27 & 0 & 6 & 63458.0 \\
\hline 176400 & 78.0110 & 150.8505 & 2348.09 & 이 & 6 & 0.0 \\
\hline 176550 & 78.0112 & 150.8441 & 2347.93 & 이 & 6 & 0.0 \\
\hline 176700 & 78.0113 & 150.8376 & 2348.12 & 이 & 6 & 63446.4 \\
\hline 176850 & 78.0114 & 150.8312 & 2348.10 & 이 & 6 & 0.0 \\
\hline 177000 & 78.0116 & 150.8248 & 2348.32 & 이 & 6 & 0.0 \\
\hline 177150 & 78.0117 & 150.8183 & 2348.58 & 이 & 6 & 63448.8 \\
\hline 177300 & 78.0118 & 150.8119 & 2348.97 & 0) & 6 & 0.0 \\
\hline 177450 & 78.0120 & 150.8055 & 2349.36 & 이 & 6 & 0.0 \\
\hline 177600 & 78.0121 & 150.7990 & 2349.42 & 이 & 6 & 63440.2 \\
\hline 177750 & 78.0123 & 150.7926 & 2349.42 & 이 & 6 & 0.0 \\
\hline 177900 & 78.0124 & 150.7861 & 2349.45 & 이 & 6 & 63435.9 \\
\hline 178050 & 78.0125 & 150.7797 & 2349.28 & 이 & 6 & 0.0 \\
\hline 178200 & 78.0127 & 150.7733 & 2349.25 & 이 & 6 & 0.0 \\
\hline 178350 & 78.0128 & 150.7668 & 2349.06 & 이 & 6 & 63430.7 \\
\hline 178500 & 78.0129 & 150.7604 & 2349.48 & 0 & 6 & 0.0 \\
\hline 178650 & 78.0131 & 150.7540 & 2348.70 & 이 & 6 & 0.0 \\
\hline 178800 & 78.0132 & 150.7475 & 2348.37 & 이 & 6 & 63427.4 \\
\hline 178950 & 78.0133 & 150.7411 & 2347.89 & 이 & 6 & 0.0 \\
\hline 179100 & 78.0135 & 150.7347 & 2347.66 & o & 6 & 0.0 \\
\hline 179250 & 78.0136 & 150.7282 & 2347.26 & 이 & 6 & 63426.3 \\
\hline 179400 & 78.0137 & 150.7218 & 2346.83 & 이 & 6 & 0.0 \\
\hline 179550 & 78.0139 & 150.7154 & 2346.36 & 이 & 6 & 0.0 \\
\hline 179700 & 78.0140 & 150.7090 & 2345.86 & 이 & 6 & 63424.5 \\
\hline
\end{tabular}




\begin{tabular}{|c|c|c|c|c|c|c|}
\hline Distance $(m)$ & $\begin{array}{l}\text { Flag Loc. } \\
\text { Latitude ('S) }\end{array}$ & $\begin{array}{c}\text { Flag Loc. } \\
\left.\text { Longitude ( }{ }^{\circ} \mathrm{E}\right)\end{array}$ & Elevation (m) & $\begin{array}{l}\text { Shot } \\
\text { Number }\end{array}$ & $\begin{array}{c}\text { Julian } \\
\text { Day }\end{array}$ & $\begin{array}{l}\text { Total Mag. } \\
\text { Field (nT) }\end{array}$ \\
\hline 179850 & 78.0742 & 150.7025 & 2345.48 & 0 & 6 & 63426.4 \\
\hline 180000 & 78.0143 & 150.6960 & 2344.93 & 0 & 6 & 0.0 \\
\hline 180150 & 78.0144 & 150.6896 & 2344.42 & 0 & 6 & 0.0 \\
\hline 180300 & 78.0146 & 150.6832 & 2343.90 & 0 & 6 & 63422.7 \\
\hline 180450 & 78.0147 & 150.6768 & 2343.59 & 0 & 6 & 0.0 \\
\hline 180600 & 78.0148 & 150.6703 & 2342.73 & 0 & 6 & 0.0 \\
\hline 180750 & 78.0150 & 150.6639 & 2342.07 & 0 & 6 & 63416.1 \\
\hline 180900 & 78.0151 & 150.6575 & 2341.90 & 0 & 6 & 0.0 \\
\hline 181050 & 78.0152 & 150.6510 & 2341.33 & 0 & 6 & 0.0 \\
\hline 181200 & 78.0154 & 150.6446 & 2340.71 & 0 & 6 & 63412.4 \\
\hline 181350 & 78.0155 & 150.6382 & 2340.47 & 0 & 6 & 0.0 \\
\hline 181500 & 78.0156 & 150.6317 & 2340.03 & 0 & 6 & 0.0 \\
\hline 181650 & 78.0158 & 150.6253 & 2339.85 & 0 & 6 & 63412.8 \\
\hline 181800 & 78.0159 & 150.6189 & 2339.39 & 0 & 6 & 0.0 \\
\hline 181950 & 78.0160 & 150.6124 & 2339.00 & 0 & 6 & 63415.7 \\
\hline 182100 & 78.0162 & 150.6059 & 2339.04 & 0 & 6 & 0.0 \\
\hline 182250 & 78.0163 & 150.5995 & 2338.88 & 0 & 6 & 0.0 \\
\hline 182400 & 78.0164 & 150.5931 & 2338.72 & 0 & 6 & 63403.5 \\
\hline 182550 & 78.0166 & 150.5866 & 2338.58 & 0 & 6 & 0.0 \\
\hline 182700 & 78.0167 & 150.5802 & 2338.67 & 0 & 6 & 0.0 \\
\hline 182850 & 78.0168 & 150.5738 & 2338.61 & 0 & 6 & 63407.4 \\
\hline 183000 & 78.0170 & 150.5673 & 2338.80 & 0 & 6 & 0.0 \\
\hline 183150 & 78.0171 & 150.5609 & 2338.74 & 0 & 6 & 0.0 \\
\hline 183300 & 78.0172 & 150.5545 & 2338.79 & 0 & 6 & 63418.3 \\
\hline 183450 & 78.0174 & 150.5480 & 2338.83 & 0 & 6 & 0.0 \\
\hline 183600 & 78.0175 & 150.5416 & 2339.02 & 0 & 6 & 0.0 \\
\hline 183750 & 78.0176 & 150.5352 & 2339.16 & 0 & 6 & 0.0 \\
\hline 183900 & 78.0178 & 150.5287 & 2339.37 & 0 & 6 & 63423.0 \\
\hline 184050 & 78.0179 & 150.5222 & 2339.45 & 0 & 6 & 0.0 \\
\hline 184200 & 78.0180 & 150.5158 & 2339.70 & 0 & 6 & 0.0 \\
\hline 184500 & 78.0182 & 150.5029 & 2340.21 & 0 & 6 & 0.0 \\
\hline 184650 & 78.0184 & 150.4965 & 2340.63 & 0 & 6 & 0.0 \\
\hline 184800 & 78.0185 & 150.4900 & 2341.09 & 0 & 6 & 63449.1 \\
\hline 184950 & 78.0186 & 150.4836 & 2341.44 & 0 & 6 & 0.0 \\
\hline 185100 & 78.0187 & 150.4771 & 2341.64 & 0 & 6 & 0.0 \\
\hline 185250 & 78.0188 & 150.4707 & 2342.08 & 0 & 6 & 63432.5 \\
\hline 185400 & 78.0189 & 150.4642 & 2342.46 & 0 & 6 & 0.0 \\
\hline 185550 & 78.0190 & 150.4578 & 2343.12 & 1336 & 8 & 63377.8 \\
\hline 185700 & 78.0191 & 150.4513 & 2343.94 & 1337 & 8 & 0.0 \\
\hline 185850 & 78.0192 & 150.4449 & 2344.50 & 1338 & 8 & 0.0 \\
\hline 186000 & 78.0193 & 150.4384 & 2345.01 & 1339 & 8 & 0.0 \\
\hline 186150 & 78.0194 & 150.4320 & 2345.96 & 1340 & 8 & 63330.2 \\
\hline 186300 & 78.0195 & 150.4255 & 2346.18 & 1341 & 8 & 0.0 \\
\hline 186450 & 78.0196 & 150.4191 & 2347.27 & 1342 & 8 & 0.0 \\
\hline 186600 & 78.0196 & 150.4126 & 2348.29 & 1343 & 8 & 0.0 \\
\hline 186750 & 78.0197 & 150.4061 & 2349.43 & 1344 & 8 & 63344.1 \\
\hline 186900 & 78.0198 & 150.3997 & 2351.41 & 1345 & 8 & 0.0 \\
\hline 187050 & 78.0199 & 150.3932 & 2352.79 & 1346 & 8 & 0.0 \\
\hline 187200 & 78.0200 & 150.3868 & 2354.29 & 1347 & 8 & 0.0 \\
\hline 187350 & 78.0201 & 150.3803 & 2356.31 & 1348 & 8 & 63332.0 \\
\hline 187500 & 78.0202 & 150.3738 & 2357.83 & 1349 & 8 & 63338.6 \\
\hline 187650 & 78.0203 & 150.3674 & 2359.07 & 1350 & 8 & 0.0 \\
\hline
\end{tabular}




\begin{tabular}{|c|c|c|c|c|c|c|}
\hline Distance (m) & $\begin{array}{l}\text { Flag Loc. } \\
\text { Latitude ('S) }\end{array}$ & $\begin{array}{c}\text { Flag Loc. } \\
\text { Longitude ( } \\
\end{array}$ & Elevation (m) & $\begin{array}{c}\text { Shot } \\
\text { Number }\end{array}$ & $\begin{array}{l}\text { Julian } \\
\text { Day }\end{array}$ & $\begin{array}{l}\text { Total Mag. } \\
\text { Field (nT) }\end{array}$ \\
\hline$\overline{787800}$ & 78.0203 & 750.3609 & 2360.22 & 1357 & $\overline{8}$ & $\overline{0.0}$ \\
\hline 187950 & 78.0204 & 150.3545 & 2361.23 & 1352 & 8 & 63365.8 \\
\hline 188100 & 78.0205 & 150.3480 & 2362.32 & 1353 & 8 & 0.0 \\
\hline 188250 & 78.0206 & 150.3416 & 2363.18 & 1354 & 8 & 0.0 \\
\hline 188400 & 78.0207 & 150.3351 & 2363.97 & 1355 & 8 & 0.0 \\
\hline 188550 & 78.0208 & 150.3287 & 2364.92 & 1356 & 8 & 63346.9 \\
\hline 188700 & 78.0209 & 150.3222 & 2365.43 & 1357 & 8 & 63354.9 \\
\hline 188850 & 78.0210 & 150.3157 & 2366.06 & 1358 & 8 & 0.0 \\
\hline 189000 & 78.0211 & 150.3093 & 2366.46 & 1359 & 8 & 0.0 \\
\hline 189150 & 78.0211 & 150.3028 & 2367.14 & 1360 & 8 & 63364.9 \\
\hline 189300 & 78.0212 & 150.2964 & 2367.45 & 1361 & 8 & 0.0 \\
\hline 189450 & 78.0213 & 150.2899 & 2367.58 & 1362 & 8 & 0.0 \\
\hline 189600 & 78.0214 & 150.2834 & 2368.17 & 1363 & 8 & 0.0 \\
\hline 189750 & 78.0215 & 150.2770 & 2368.42 & 1364 & 8 & 63350.1 \\
\hline 189900 & 78.0216 & 150.2705 & 2368.69 & 1365 & 8 & 0.0 \\
\hline 190050 & 78.0217 & 150.2641 & 2368.96 & 1366 & 8 & 0.0 \\
\hline 190200 & 78.0218 & 150.2576 & 2369.25 & 1367 & 8. & 0.0 \\
\hline 190350 & 78.0218 & 150.2512 & 2369.32 & 1368 & 8 & 63333.0 \\
\hline 190500 & 78.0219 & 150.2447 & 2369.62 & 1369 & 8 & 0.0 \\
\hline 190650 & 78.0220 & 150.2383 & 2370.07 & 1370 & 8 & 0.0 \\
\hline 190800 & 78.0221 & 150.2318 & 2370.90 & 1371 & 8 & 63330.8 \\
\hline 190950 & 78.0222 & 150.2253 & 2371.13 & 1372 & 8 & 63343.5 \\
\hline 191100 & 78.0223 & 150.2189 & 2371.85 & 1373 & 8 & 0.0 \\
\hline 191250 & 78.0224 & 150.2124 & 2371.97 & 1374 & 8 & 0.0 \\
\hline 191400 & 78.0225 & 150.2059 & 2372.52 & 1375 & 8 & 0.0 \\
\hline 191550 & 78.0226 & 150.1995 & 2373.25 & 1376 & 8 & 63357.3 \\
\hline 191700 & 78.0226 & 150.1930 & 2373.99 & 1377 & 8 & 0.0 \\
\hline 191850 & 78.0227 & 150.1866 & 2374.36 & 1378 & 8 & 0.0 \\
\hline 192000 & 78.0228 & 150.1801 & 2374.96 & 1379 & 8 & 0.0 \\
\hline 192150 & 78.0229 & 150.1737 & 2375.39 & 1380 & 8 & 63348.8 \\
\hline 192300 & 78.0230 & 150.1672 & 2375.99 & 1381 & 8 & 0.0 \\
\hline 192450 & 78.0231 & 150.1608 & 2376.39 & 1382 & 8 & 0.0 \\
\hline 192600 & 78.0232 & 150.1543 & 2376.68 & 1383 & 8 & 0.0 \\
\hline 192750 & 78.0232 & 150.1478 & 2376.77 & 1384 & 8 & 63380.9 \\
\hline 192900 & 78.0233 & 150.1413 & 2377.07 & 1385 & 8 & 63387.7 \\
\hline 193050 & 78.0234 & 150.1349 & 2377.26 & 1386 & 8 & 0.0 \\
\hline 193200 & 78.0235 & 150.1284 & 2377.40 & 1387 & 8 & 0.0 \\
\hline 193350 & 78.0236 & 150.1220 & 2378.12 & 1388 & 8 & 63391.8 \\
\hline 193500 & 78.0237 & 150.1155 & 2378.88 & 1389 & 8 & 0.0 \\
\hline 193650 & 78.0238 & 150.1091 & 2378.51 & 1390 & 8 & 0.0 \\
\hline 193800 & 78.0238 & 150.1026 & 2378.46 & 1391 & 8 & 0.0 \\
\hline 193950 & 78.0239 & 150.0961 & 2378.70 & 1392 & 8 & 63405.0 \\
\hline 194100 & 78.0240 & 150.0897 & 2378.99 & 1393 & 8 & 0.0 \\
\hline 194250 & 78.0241 & 150.0832 & 2378.84 & 1394 & 8 & 0.0 \\
\hline 194400 & 78.0242 & 150.0768 & 2378.86 & 1395 & 8 & 0.0 \\
\hline 194550 & 78.0243 & 150.0703 & 2379.04 & 1396 & 8 & 63408.4 \\
\hline 194700 & 78.0244 & 150.0639 & 2379.10 & 1397 & 8 & 0.0 \\
\hline 194850 & 78.0244 & 150.0574 & 2379.01 & 1398 & 8 & 0.0 \\
\hline 195000 & 78.0245 & 150.0509 & 2378.92 & 1399 & 8 & 63427.2 \\
\hline 195150 & 78.0246 & 150.0444 & 2378.93 & 1400 & 8 & 0.0 \\
\hline 195300 & 78.0247 & 150.0380 & 2379.11 & 1401 & 8 & 0.0 \\
\hline 195450 & 78.0248 & 150.0315 & 2379.37 & 1402 & 9 & 63375.3 \\
\hline
\end{tabular}




\begin{tabular}{|c|c|c|c|c|c|c|}
\hline Distance $(m)$ & $\begin{array}{c}\text { Flag Loc. } \\
\left.\text { Latitude ( }{ }^{\circ} \mathbf{S}\right)\end{array}$ & $\begin{array}{c}\text { Flag Loc. } \\
\text { Longitude }\left({ }^{\circ} \mathrm{E}\right)\end{array}$ & Elevation (m) & $\begin{array}{c}\text { Shot } \\
\text { Number }\end{array}$ & $\begin{array}{c}\text { Julian } \\
\text { Day }\end{array}$ & $\begin{array}{l}\text { Total Mag. } \\
\text { Field (nT) }\end{array}$ \\
\hline 195600 & 78.0249 & 150.0257 & 2379.35 & 1403 & $\overline{9}$ & $\overline{0.0}$ \\
\hline 195750 & 78.0250 & 150.0186 & 2379.35 & 1404 & 9 & 0.0 \\
\hline 195900 & 78.0250 & 150.0121 & 2379.30 & 1405 & 9 & 0.0 \\
\hline 196050 & 78.0251 & 150.0057 & 2379.36 & 1406 & 9 & 63371.5 \\
\hline 196200 & 78.0252 & 149.9992 & 2379.84 & 1407 & 9 & 0.0 \\
\hline 196350 & 78.0253 & 149.9928 & 2380.01 & 1408 & 9 & 0.0 \\
\hline 196500 & 78.0254 & 149.9863 & 2380.33 & 1409 & 9 & 0.0 \\
\hline 196650 & 78.0255 & 149.9799 & 2380.33 & 1410 & 9 & 63394.5 \\
\hline 196800 & 78.0256 & 149.9734 & 2380.59 & 1411 & 9 & 0.0 \\
\hline 196950 & 78.0256 & 149.9669 & 2380.99 & 1412 & 9 & 0.0 \\
\hline 197100 & 78.0257 & 149.9604 & 2381.49 & 1413 & 9 & 63385.3 \\
\hline 197250 & 78.0258 & 149.9540 & 2381.44 & 1414 & 9 & 63377.4 \\
\hline 197400 & 78.0259 & 149.9475 & 2382.05 & 1415 & 9 & 0.0 \\
\hline 197550 & 78.0260 & 149.9411 & 2382.36 & 1416 & 9 & 0.0 \\
\hline 197700 & 78.0261 & 149.9346 & 2382.64 & 1417 & 9 & 0.0 \\
\hline 197850 & 78.0262 & 149.9282 & 2382.87 & 1418 & 9 & 63386.9 \\
\hline 198000 & 78.0262 & 149.9217 & 2383.20 & 1419 & 9 & 0.0 \\
\hline 198150 & 78.0263 & 149.9152 & 2383.38 & 1420 & 9 & 0.0 \\
\hline 198300 & 78.0264 & 149.9088 & 2383.97 & 1421 & 9 & 0.0 \\
\hline 198450 & 78.0265 & 149.9023 & 2384.24 & 1422 & 9 & 63373.7 \\
\hline 198600 & 78.0266 & 149.8959 & 2384.67 & 1423 & 9 & 0.0 \\
\hline 198750 & 78.0266 & 149.8894 & 2384.81 & 1424 & 9 & 0.0 \\
\hline 198900 & 78.0267 & 149.8829 & 2385.36 & 1425 & 9 & 0.0 \\
\hline 199050 & 78.0268 & 149.8765 & 2385.70 & 1426 & 9 & 63371.1 \\
\hline 199200 & 78.0269 & 149.8700 & 2386.11 & 1427 & 9 & 63369.0 \\
\hline 199350 & 78.0270 & 149.8635 & 2386.70 & 1428 & 9 & $0 . c$ \\
\hline 199500 & 78.0271 & 149.8571 & 2386.76 & 1429 & 9 & 0.0 \\
\hline 199650 & 78.0272 & 149.8506 & 2386.98 & 1430 & 9 & 63363.7 \\
\hline 199800 & 78.0272 & 149.8441 & 2387.30 & 1431 & 9 & 0.0 \\
\hline 199950 & 78.0273 & 149.8377 & 2388.06 & 1432 & 9 & 0.0 \\
\hline 200100 & 78.0274 & 149.8312 & 2388.07 & 1433 & 9 & 0.0 \\
\hline 200250 & 78.0275 & 149.8248 & 2388.12 & 1434 & 9 & 63364.2 \\
\hline 200400 & 78.0276 & 149.8183 & 2388.53 & 1435 & 9 & 0.0 \\
\hline 200550 & 78.0277 & 149.8118 & 2388.96 & 1436 & 9 & 0.0 \\
\hline 200700 & 78.0277 & 149.8054 & 2389.16 & 1437 & 9 & 0.0 \\
\hline 200850 & 78.0278 & 149.7989 & 2389.50 & 1438 & 9 & 63368.2 \\
\hline 201000 & 78.0279 & 149.7925 & 2389.41 & 1439 & 9 & 0.0 \\
\hline 201150 & 78.0280 & 149.7860 & 2389.82 & 1440 & 9 & 0.0 \\
\hline 201300 & 78.0281 & 149.7795 & 2390.18 & 1441 & 9 & 63378.9 \\
\hline 201450 & 78.0282 & 149.7730 & 2390.46 & 1442 & 9 & 0.0 \\
\hline 201600 & 78.0283 & 149.7666 & 2390.72 & 1443 & 9 & 0.0 \\
\hline 201750 & 78.0283 & 149.7601 & 2391.03 & 1444 & 9 & 0.0 \\
\hline 201900 & 78.0284 & 149.7537 & 2391.12 & 1445 & 9 & 0.0 \\
\hline 202050 & 78.0285 & 149.7472 & 2391.35 & 1446 & 9 & 63387.2 \\
\hline 202200 & 78.0286 & 149.7407 & 2391.66 & 1447 & 9 & 0.0 \\
\hline 202350 & 78.0287 & 149.7343 & 2391.79 & 1448 & 9 & 0.0 \\
\hline 202500 & 78.0287 & 149.7278 & 2392.31 & 1449 & 9 & 0.0 \\
\hline 202650 & 78.0288 & 149.7214 & 2392.54 & 1450 & 9 & 63399.8 \\
\hline 202800 & 78.0289 & 149.7149 & 2392.52 & 1451 & 9 & 0.0 \\
\hline 202950 & 78.0290 & 149.7084 & 2392.80 & 1452 & 9 & 0.0 \\
\hline 203100 & 78.0291 & 149.7020 & 2393.06 & 1453 & 9 & 0.0 \\
\hline 203250 & 78.0292 & 149.6955 & 2393.22 & 1454 & 9 & 63403.6 \\
\hline
\end{tabular}




\begin{tabular}{|c|c|c|c|c|c|c|}
\hline Distance (m) & $\begin{array}{l}\text { Flag Loc. } \\
\text { Latitude ('S) }\end{array}$ & $\begin{array}{c}\text { Flag Loc. } \\
\text { Longitude }\left({ }^{\circ} \mathrm{E}\right)\end{array}$ & Elevation (m) & $\begin{array}{l}\text { Shot } \\
\text { Number }\end{array}$ & $\begin{array}{c}\text { Julian } \\
\text { Day }\end{array}$ & $\begin{array}{l}\text { Total Mag. } \\
\text { Field (nT) }\end{array}$ \\
\hline 203400 & 78.0293 & 749.6890 & 2393.90 & 7455 & $\overline{9}$ & 63407.3 \\
\hline 203550 & 78.0293 & 149.6826 & 2393.91 & 1456 & 9 & 0.0 \\
\hline 203700 & 78.0294 & 149.6761 & 2394.19 & 1457 & 9 & 0.0 \\
\hline 203850 & 78.0295 & 149.6696 & 2394.20 & 1458 & 9 & 63428.4 \\
\hline 204000 & 78.0296 & 149.6632 & 2394.56 & 1459 & 9 & 0.0 \\
\hline 204150 & 78.0297 & 149.6567 & 2394.28 & 1460 & 9 & 0.0 \\
\hline 204300 & 78.0297 & 149.6503 & 2394.63 & 1461 & 9 & 0.0 \\
\hline 204450 & 78.0298 & 149.6438 & 2394.98 & 1462 & 9 & 63435.1 \\
\hline 204600 & 78.0299 & 149.6373 & 2394.86 & 1463 & 9 & 0.0 \\
\hline 204750 & 78.0300 & 149.6309 & 2394.68 & 1464 & 9 & 0.0 \\
\hline 204900 & 78.0301 & 149.6244 & 2395.18 & 1465 & 9 & 0.0 \\
\hline 205050 & 78.0302 & 149.6180 & 2395.03 & 1466 & 9 & 63431.1 \\
\hline 205200 & 78.0302 & 149.6115 & 2395.08 & 1467 & 9 & 0.0 \\
\hline 205350 & 78.0303 & 149.6050 & 2395.28 & 1468 & 9 & 0.0 \\
\hline 205500 & 78.0304 & 149.5985 & 2395.18 & 1469 & 9 & 63422.2 \\
\hline 205650 & 78.0305 & 149.5921 & 2395.29 & 1470 & 10 & 63435.9 \\
\hline 205800 & 78.0306 & 149.5856 & 2395.35 & 1471 & 10 & 0.0 \\
\hline 205950 & 78.0307 & 149.5791 & 2395.42 & 1472 & 10 & 0.0 \\
\hline 206100 & 78.0307 & 149.5727 & 2395.35 & 1473 & 10 & 0.0 \\
\hline 206250 & 78.0308 & 149.5662 & 2395.70 & 1474 & 10 & 63435.4 \\
\hline 206400 & 78.0309 & 149.5598 & 2395.60 & 1475 & 10 & 0.0 \\
\hline 206550 & 78.0310 & 149.5533 & 2395.67 & 1476 & 10 & 0.0 \\
\hline 206700 & 78.0311 & 149.5468 & 2395.43 & 1477 & 10 & 0.0 \\
\hline 206850 & 78.0311 & 149.5404 & 2395.03 & 1478 & 10 & 63433.5 \\
\hline 207000 & 78.0312 & 149.5339 & 2395.13 & 1479 & 10 & 0.0 \\
\hline 207150 & 78.0313 & 149.5274 & 2395.19 & 1480 & 10 & 0.0 \\
\hline 207300 & 78.0314 & 149.5210 & 2395.36 & 1481 & 10 & 0.0 \\
\hline 207450 & 78.0315 & 149.5145 & 2395.29 & 1482 & 10 & 63426.4 \\
\hline 207600 & 78.0316 & 149.5080 & 2395.45 & 1483 & 10 & 63416.1 \\
\hline 207750 & 78.0316 & 149.5016 & 2395.19 & 1484 & 10 & 0.0 \\
\hline 207900 & 78.0317 & 149.4951 & 2395.50 & 1485 & 10 & 0.0 \\
\hline 208050 & 78.0318 & 149.4886 & 2395.54 & 1486 & 10 & 0.0 \\
\hline 208200 & 78.0319 & 149.4822 & 2395.56 & 1487 & 10 & 63430.8 \\
\hline 208350 & 78.0320 & 149.4757 & 2395.77 & 1488 & 10 & 0.0 \\
\hline 208500 & 78.0320 & 149.4692 & 2395.77 & 1489 & 10 & 0.0 \\
\hline 208650 & 78.0321 & 149.4628 & 2395.68 & 1490 & 10 & 0.0 \\
\hline 208800 & 78.0322 & 149.4563 & 2396.01 & 1491 & 10 & 63416.3 \\
\hline 208950 & 78.0323 & 149.4498 & 2395.92 & 1492 & 10 & 0.0 \\
\hline 209100 & 78.0324 & 149.4434 & 2395.75 & 1493 & 10 & 0.0 \\
\hline 209250 & 78.0324 & 149.4369 & 2396.04 & 1494 & 10 & 0.0 \\
\hline 209400 & 78.0325 & 149.4305 & 2396.21 & 1495 & 10 & 63390.3 \\
\hline 209550 & 78.0326 & 149.4240 & 2396.21 & 1496 & 10 & 63375.7 \\
\hline 209700 & 78.0327 & 149.4175 & 2396.47 & 1497 & 10 & 0.0 \\
\hline 209850 & 78.0328 & 149.4110 & 2396.32 & 1498 & 10 & 63391.8 \\
\hline 210000 & 78.0329 & 149.4046 & 2396.93 & 1499 & 10 & 0.0 \\
\hline 210150 & 78.0329 & 149.3981 & 2396.61 & 1500 & 10 & 0.0 \\
\hline 210300 & 78.0330 & 149.3916 & 2396.67 & 1501 & 10 & 0.0 \\
\hline 210450 & 78.0331 & 149.3852 & 2396.77 & 1502 & 10 & 63375.7 \\
\hline 210600 & 78.0332 & 149.3787 & 2396.87 & 1503 & 10 & 0.0 \\
\hline 210750 & 78.0333 & 149.3723 & 2396.74 & 1504 & 10 & 0.0 \\
\hline 210900 & 78.0333 & 149.3658 & 2396.92 & 1505 & 10 & 0.0 \\
\hline 211050 & 78.0334 & 149.3593 & 2396.81 & 1506 & 10 & 63343.2 \\
\hline
\end{tabular}




\begin{tabular}{|c|c|c|c|c|c|c|}
\hline Distance $(\mathrm{m})$ & $\begin{array}{c}\text { Flag Loc. } \\
\left.\text { Latitude ( }{ }^{\circ} \mathrm{S}\right)\end{array}$ & $\begin{array}{c}\text { Flag Loc. } \\
\left.\text { Longitude ( }{ }^{\circ} \mathrm{E}\right)\end{array}$ & $\mid$ Elevation $(\mathbf{m}) \mid$ & $\begin{array}{c}\text { Shot } \\
\text { Number }\end{array}$ & $\begin{array}{c}\text { Julian } \\
\text { Day }\end{array}$ & $\begin{array}{l}\text { Total Mag. } \\
\text { Field (nT) }\end{array}$ \\
\hline 211200 & 78.0335 & 749.3529 & $\overline{2397.13}$ & 7507 & T0 & $\overline{0.0}$ \\
\hline 211350 & 78.0338 & 149.3270 & 2397.29 & 1508 & 10 & 0.0 \\
\hline 211500 & 78.0337 & 149.3399 & 2397.31 & 1509 & 10 & 63333.3 \\
\hline 211650 & 78.0338 & 149.3334 & 2397.12 & 1510 & 10 & 63333.6 \\
\hline 211950 & 78.0339 & 149.3205 & 2397.35 & 1512 & 10 & 0.0 \\
\hline 212100 & 78.0340 & 149.3140 & 2397.55 & 1513 & 10 & 63338.8 \\
\hline 212250 & 78.0341 & 149.3076 & 2397.73 & 1514 & 10 & 63341.1 \\
\hline 212400 & 78.0342 & 149.3011 & 2398.01 & 1515 & 10 & 0.0 \\
\hline 212550 & 78.0342 & 149.2946 & 2398.27 & 1516 & 10 & 0.0 \\
\hline 212700 & 78.0343 & 149.2882 & 2398.22 & 1517 & 10 & 0.0 \\
\hline 212850 & 78.0344 & 149.2817 & 2398.18 & 1518 & 10 & 63342.3 \\
\hline 213000 & 78.0345 & 149.2753 & 2398.31 & 1519 & 10 & 0.0 \\
\hline 213150 & 78.0346 & 149.2688 & 2398.26 & 1520 & 10 & 0.0 \\
\hline 213300 & 78.0346 & 149.2623 & 2398.52 & 1521 & 10 & 0.0 \\
\hline 213450 & 78.0347 & 149.2559 & 2398.17 & 1522 & 10 & 63334.9 \\
\hline 213600 & 78.0348 & 149.2494 & 2398.22 & 1523 & 10 & 0.0 \\
\hline 213750 & 78.0349 & 149.2429 & 2397.99 & 1524 & 10 & 0.0 \\
\hline 213900 & 78.0350 & 149.2364 & 2398.15 & 1525 & 10 & 63327.7 \\
\hline 214050 & 78.0351 & 149.2300 & 2397.91 & 1526 & 10 & 63319.2 \\
\hline 214200 & 78.0351 & 149.2235 & 2398.02 & 1527 & 10 & 0.0 \\
\hline 214350 & 78.0352 & 149.2170 & 2397.93 & 1528 & 10 & 0.0 \\
\hline 214500 & 78.0353 & 149.2106 & 2397.67 & 1529 & 10 & 0.0 \\
\hline 214650 & 78.0354 & 149.2041 & 2397.41 & 1530 & 10 & 63295.7 \\
\hline 214800 & 78.0355 & 149.1976 & 2397.21 & 1531 & 10 & 0.0 \\
\hline 214950 & 78.0355 & 149.1912 & 2397.26 & 1532 & 10 & 0.0 \\
\hline 215100 & 78.0356 & 149.1847 & 2397.12 & 1533 & 10 & 0.0 \\
\hline 215250 & 78.0357 & 149.1782 & 2396.88 & 1534 & 10 & 63275.6 \\
\hline 215400 & 78.0358 & 149.1718 & 2396.95 & 1535 & 10 & 0.0 \\
\hline 215550 & 78.0359 & 149.1653 & 2396.58 & 1536 & 10 & 0.0 \\
\hline 215700 & 78.0359 & 149.1588 & 2396.42 & 1537 & 10 & 0.0 \\
\hline 215850 & 78.0360 & 149.1524 & 2396.61 & 1538 & 10 & 63265.7 \\
\hline 216000 & 78.0361 & 149.1459 & 2396.60 & 1539 & 10 & 63258.9 \\
\hline 216150 & 78.0362 & 149.1394 & 2396.44 & 1540 & 12 & 63102.9 \\
\hline 216300 & 78.0363 & 149.1329 & 2396.43 & 1541 & 12 & 0.0 \\
\hline 216450 & 78.0363 & 149.1265 & 2396.35 & 1542 & 12 & 63102.0 \\
\hline 216600 & 78.0364 & 149.1200 & 2396.32 & 1543 & 12 & 0.0 \\
\hline 216750 & 78.0365 & 149.1135 & 2396.34 & 1544 & 12 & 0.0 \\
\hline 216900 & 78.0366 & 149.1071 & 2396.61 & 1545 & 12 & 0.0 \\
\hline 217050 & 78.0367 & 149.1006 & 2396.52 & 1546 & 12 & 63102.9 \\
\hline 217200 & 78.0367 & 149.0941 & 2396.70 & 1547 & 12 & 0.0 \\
\hline 217350 & 78.0368 & 149.0877 & 2396.88 & 1548 & 12 & 0.0 \\
\hline 217500 & 78.0369 & 149.0812 & 2397.23 & 1549 & 12 & 63114.7 \\
\hline 217650 & 78.0370 & 149.0747 & 2397.48 & 1550 & 12 & 63108.6 \\
\hline 217800 & 78.0370 & 149.0683 & 2397.44 & 1551 & 12 & 0.0 \\
\hline 217950 & 78.0371 & 149.0618 & 2397.85 & 1552 & 12 & 0.0 \\
\hline 218100 & 78.0372 & 149.0553 & 2397.92 & 1553 & 12 & 63127.2 \\
\hline 218250 & 78.0373 & 149.0488 & 2398.44 & 1554 & 12 & 63108.0 \\
\hline 218400 & 78.0374 & 149.0424 & 2398.59 & 1555 & 12 & 0.0 \\
\hline 218550 & 78.0374 & 149.0359 & 2399.02 & 1556 & 12 & 0.0 \\
\hline 218700 & 78.0375 & 149.0294 & 2399.18 & 1557 & 12 & 0.0 \\
\hline 218850 & 78.0376 & 149.0230 & 2399.33 & 1558 & 12 & 63073.0 \\
\hline 219000 & 78.0377 & 149.0165 & 2399.64 & 1559 & 12 & 0.0 \\
\hline
\end{tabular}




\begin{tabular}{|c|c|c|c|c|c|c|}
\hline Distance $(m)$ & $\begin{array}{l}\text { Flag Loc. } \\
\text { Latitude ('S) }\end{array}$ & $\begin{array}{c}\text { Flag Loc. } \\
\left.\text { Longitude ( }{ }^{\circ} \mathrm{E}\right)\end{array}$ & Elevation (m) & $\begin{array}{c}\text { Shot } \\
\text { Number }\end{array}$ & $\begin{array}{c}\text { Julian } \\
\text { Day }\end{array}$ & $\begin{array}{l}\text { Total Mag. } \\
\text { Field (nT) }\end{array}$ \\
\hline 219750 & 78.0378 & 749.0100 & 2400.26 & 7560 & 12 & $\overline{0.0}$ \\
\hline 219300 & 78.0378 & 149.0036 & 2400.55 & 1561 & 12 & 0.0 \\
\hline 219450 & 78.0379 & 148.9971 & 2400.93 & 1562 & 12 & 0.0 \\
\hline 219600 & 78.0380 & 148.9906 & 2401.32 & 1563 & 12 & 0.0 \\
\hline 219750 & 78.0381 & 148.9842 & 2401.30 & 1564 & 12 & 0.0 \\
\hline 219900 & 78.0381 & 148.9777 & 2401.68 & 1565 & 12 & 0.0 \\
\hline 220050 & 78.0382 & 148.9712 & 2402.23 & 1566 & 12 & 63123.9 \\
\hline 220200 & 78.0383 & 148.9647 & 2402.75 & 1567 & 12 & 63147.5 \\
\hline 220350 & 78.0384 & 148.9583 & 2403.21 & 1568 & 12 & 0.0 \\
\hline 220500 & 78.0385 & 148.9518 & 2403.13 & 1569 & 12 & 0.0 \\
\hline 220650 & 78.0385 & 148.9453 & 2403.66 & 1570 & 12 & 63193.7 \\
\hline 220800 & 78.0386 & 148.9389 & 2404.24 & 1571 & 12 & 0.0 \\
\hline 220950 & 78.0387 & 148.9324 & 2404.35 & 1572 & 12 & 0.0 \\
\hline 221100 & 78.0388 & 148.9259 & 2404.80 & 1573 & 12 & 0.0 \\
\hline 221250 & 78.0391 & 148.9001 & 2405.54 & 1574 & 12 & 63164.7 \\
\hline 221400 & 78.0392 & 148.8936 & 2405.92 & 1575 & 12 & 0.0 \\
\hline 221550 & 78.0390 & 148.9065 & 2405.45 & 1576 & 12 & 0.0 \\
\hline 222000 & 78.0392 & 148.8871 & 2405.83 & 1579 & 12 & 0.0 \\
\hline 222150 & 78.0393 & 148.8807 & 2406.03 & 1580 & 12 & 0.0 \\
\hline 222300 & 78.0394 & 148.8741 & 2406.62 & 1581 & 12 & 63253.0 \\
\hline 222450 & 78.0395 & 148.8677 & 2406.62 & 1582 & 12 & 63257.2 \\
\hline 222600 & 78.0396 & 148.8612 & 2407.05 & 1583 & 12 & 0.0 \\
\hline 222750 & 78.0396 & 148.8548 & 2407.16 & 1584 & 12 & 0.0 \\
\hline 222900 & 78.0397 & 148.8483 & 2407.58 & 1585 & 12 & 0.0 \\
\hline 223050 & 78.0398 & 148.8418 & 2407.69 & 1586 & 12 & 63230.3 \\
\hline 223200 & 78.0399 & 148.8353 & 2408.37 & 1587 & 12 & 0.0 \\
\hline 223350 & 78.0399 & 148.8289 & 2408.22 & 1588 & 12 & 0.0 \\
\hline 223500 & 78.0400 & 148.8224 & 2408.46 & 1589 & 12 & 0.0 \\
\hline 223650 & 78.0401 & 148.8159 & 2408.70 & 1590 & 12 & 63207.2 \\
\hline 223800 & 78.0402 & 148.8095 & 2409.12 & 1591 & 12 & 0.0 \\
\hline 223950 & 78.0402 & 148.8030 & 2409.46 & 1592 & 12 & 0.0 \\
\hline 224100 & 78.0403 & 148.7965 & 2409.84 & 1593 & 12 & 0.0 \\
\hline 224250 & 78.0404 & 148.7901 & 2410.13 & 1594 & 12 & 63229.9 \\
\hline 224400 & 78.0405 & 148.7836 & 2410.84 & 1595 & 12 & 63240.8 \\
\hline 224550 & 78.0405 & 148.7771 & 2411.09 & 1596 & 12 & 0.0 \\
\hline 224700 & 78.0406 & 148.7706 & 2411.54 & 1597 & 12 & 0.0 \\
\hline 224850 & 78.0406 & 148.7641 & 2411.70 & 1598 & 12 & 63205.4 \\
\hline 225000 & 78.0406 & 148.7576 & 2411.98 & 1599 & 12 & 0.0 \\
\hline 225150 & 78.0407 & 148.7512 & 2412.25 & 1600 & 12 & 0.0 \\
\hline 225300 & 78.0407 & 148.7447 & 2412.64 & 1601 & 12 & 0.0 \\
\hline 225450 & 78.0407 & 148.7382 & 2412.96 & 1602 & 12 & 63197.2 \\
\hline 225600 & 78.0408 & 148.7317 & 2413.28 & 1603 & 12 & 0.0 \\
\hline 225750 & 78.0408 & 148.7253 & 2413.31 & 1604 & 12 & 0.0 \\
\hline 225900 & 78.0408 & 148.7188 & 2413.79 & 1605 & 12 & 0.0 \\
\hline 226050 & 78.0409 & 148.7123 & 2414.01 & 1606 & 12 & 63192.6 \\
\hline 226200 & 78.0409 & 148.7058 & 2414.28 & 1607 & 12 & 0.0 \\
\hline 226350 & 78.0409 & 148.6994 & 2414.32 & 1608 & 12 & 0.0 \\
\hline 226500 & 78.0410 & 148.6928 & 2414.39 & 1609 & 12 & 63196.3 \\
\hline 226650 & 78.0410 & 148.6864 & 2414.55 & 1610 & 12 & 63210.0 \\
\hline 226800 & 78.0411 & 148.6799 & 2414.52 & 1611 & 12 & 0.0 \\
\hline 226950 & 78.0411 & 148.6734 & 2414.85 & 1612 & 12 & 0.0 \\
\hline 227100 & 78.0411 & 148.6669 & 2414.87 & 1613 & 12 & 0.0 \\
\hline
\end{tabular}




\begin{tabular}{|c|c|c|c|c|c|c|}
\hline Distance (m) & $\begin{array}{c}\text { Flag Loc. } \\
\left.\text { Latitude ( }{ }^{\circ} \mathrm{S}\right)\end{array}$ & $\begin{array}{c}\text { Flag Loc. } \\
\text { Longitude }\left({ }^{\circ} \mathrm{E}\right)\end{array}$ & Elevation (m) & $\begin{array}{c}\text { Shot } \\
\text { Number }\end{array}$ & $\begin{array}{c}\text { Julian } \\
\text { Day }\end{array}$ & $\begin{array}{l}\text { Total Mag. } \\
\text { Field (nT) }\end{array}$ \\
\hline 227250 & 78.0412 & 148.6604 & 2415.12 & 1614 & $\sqrt{2}$ & 63224.4 \\
\hline 227400 & 78.0412 & 148.6540 & 2415.00 & 1615 & 12 & 0.0 \\
\hline 227550 & 78.0412 & 148.6475 & 2415.08 & 1616 & 12 & 0.0 \\
\hline 227700 & 78.0413 & 148.6410 & 2415.22 & 1617 & 12 & 0.0 \\
\hline 227850 & 78.0413 & 148.6345 & 2415.04 & 1618 & 13 & 63128.1 \\
\hline 228000 & 78.0413 & 148.6281 & 2415.43 & 1619 & 13 & 0.0 \\
\hline 228150 & 78.0414 & 148.6216 & 2415.48 & 1620 & 13 & 0.0 \\
\hline 228300 & 78.0414 & 148.6151 & 2415.14 & 1621 & 13 & 0.0 \\
\hline 228450 & 78.0414 & 148.6086 & 2415.59 & 1622 & 13 & 63204.5 \\
\hline 228600 & 78.0415 & 148.6021 & 2415.38 & 1623 & 13 & 63187.9 \\
\hline 228750 & 78.0415 & 148.5956 & 2415.56 & 1624 & 13 & $0 . c$ \\
\hline 228900 & 78.0415 & 148.5892 & 2415.68 & 1625 & 13 & 0.0 \\
\hline 229050 & 78.0416 & 148.5827 & 2415.59 & 1626 & 13 & 63163.1 \\
\hline 229200 & 78.0416 & 148.5762 & 2415.72 & 1627 & 13 & 0.0 \\
\hline 229350 & 78.0416 & 148.5697 & 2415.85 & 1628 & 13 & 0.0 \\
\hline 229500 & 78.0417 & 148.5632 & 2415.89 & 1629 & 13 & 0.0 \\
\hline 229650 & 78.0417 & 148.5568 & 2416.20 & 1630 & 13 & 63167.4 \\
\hline 229800 & 78.0418 & 148.5503 & 2416.21 & 1631 & 13 & 0.0 \\
\hline 229950 & 78.0418 & 148.5438 & 2416.18 & 1632 & 13 & 0.0 \\
\hline 230100 & 78.0418 & 148.5373 & 2416.31 & 1633 & 13 & 0.0 \\
\hline 230250 & 78.0419 & 148.5309 & 2416.43 & 1634 & 13 & 63202.0 \\
\hline 230400 & 78.0419 & 148.5244 & 2416.54 & 1635 & 13 & 0.0 \\
\hline 230550 & 78.0419 & 148.5179 & 2416.64 & 1636 & 13 & 0.0 \\
\hline 230700 & 78.0420 & 148.5114 & 2416.85 & 1637 & 13 & 63205.7 \\
\hline 230850 & 78.0420 & 148.5049 & 2417.10 & 1638 & 13 & 63189.8 \\
\hline 231000 & 78.0420 & 148.4984 & 2417.22 & 1639 & 13 & 0.0 \\
\hline 231150 & 78.0421 & 148.4920 & 2417.43 & 1640 & 13 & 0.0 \\
\hline 231300 & 78.0422 & 148.4660 & 2417.78 & 1641 & 13 & 0.0 \\
\hline 231450 & 78.0422 & 148.4790 & 2417.66 & 1642 & 13 & 63204.8 \\
\hline 231600 & 78.0422 & 148.4725 & 2417.51 & 1643 & 13 & 0.0 \\
\hline 231900 & 78.0423 & 148.4596 & 2417.94 & 1645 & 13 & 0.0 \\
\hline 232050 & 78.0423 & 148.4531 & 2418.19 & 1646 & 13 & 63192.7 \\
\hline 232200 & 78.0423 & 148.4466 & 2418.17 & 1647 & 13 & 0.0 \\
\hline 232350 & 78.0424 & 148.4401 & 2418.04 & 1648 & 13 & 0.0 \\
\hline 232500 & 78.0424 & 148.4337 & 2418.20 & 1649 & 13 & 0.0 \\
\hline 232650 & 78.0424 & 148.4272 & 2418.52 & 1650 & 13 & 63184.5 \\
\hline 232800 & 78.0425 & 148.4207 & 2418.53 & 1651 & 13 & 63182.6 \\
\hline 232950 & 78.0425 & 148.4142 & 2418.43 & 1652 & 13 & 0.0 \\
\hline 233100 & 78.0425 & 148.4077 & 2418.7 이 & 1653 & 13 & 0.0 \\
\hline 233250 & 78.0426 & 148.4012 & 2418.76 & 1654 & 13 & 63195.7 \\
\hline 233400 & 78.0426 & 148.3947 & 2418.98 & 1655 & 13 & 0.0 \\
\hline 233550 & 78.0426 & 148.3883 & 2418.96 & 1656 & 13 & 0.0 \\
\hline 233700 & 78.0427 & 148.3818 & 2419.35 & 1657 & 13 & 0.0 \\
\hline 233850 & 78.0427 & 148.3753 & 2419.46 & 1658 & 13 & 63183.0 \\
\hline 234000 & 78.0427 & 148.3688 & 2419.77 & 1659 & 13 & 0.0 \\
\hline 234150 & 78.0428 & 148.3624 & 2419.67 & 1660 & 13 & 0.0 \\
\hline 234300 & 78.0428 & 148.3559 & 2420.17 & 1661 & 13 & 0.0 \\
\hline 234450 & 78.0428 & 148.3494 & 2420.33 & 1662 & 13 & 63177.8 \\
\hline 234600 & 78.0429 & 148.3429 & 2420.30 & 1663 & 13 & 0.0 \\
\hline 234750 & 78.0429 & 148.3364 & 2420.43 & 1664 & 13 & 0.0 \\
\hline 234900 & 78.0429 & 148.3299 & 2420.82 & 1665 & 13 & 63187.0 \\
\hline 235050 & 78.0430 & 148.3234 & 2421.27 & 1666 & 13 & 63193.7 \\
\hline
\end{tabular}




\begin{tabular}{|c|c|c|c|c|c|c|}
\hline Distance (m) & $\begin{array}{l}\text { Flag Loc. } \\
\left.\text { Latitude (' }{ }^{\circ}\right)\end{array}$ & $\begin{array}{c}\text { Flag Loc. } \\
\left.\text { Longitude ( }{ }^{\circ} \mathrm{E}\right)\end{array}$ & Elevation $(m)$ & $\begin{array}{l}\text { Shot } \\
\text { Number }\end{array}$ & $\begin{array}{c}\text { Julian } \\
\text { Day }\end{array}$ & $\begin{array}{l}\text { Total Mag. } \\
\text { Fiold (nT) }\end{array}$ \\
\hline$\overline{235200}$ & 78.0430 & $\overline{148.3170}$ & 2421.71 & 1667 & $\overline{\overline{73}}$ & $\overline{0.0}$ \\
\hline 235350 & 78.0431 & 148.3105 & 2421.83 & 1668 & 13 & 0.0 \\
\hline 235500 & 78.0431 & 148.3040 & 2422.03 & 1669 & 13 & 0.0 \\
\hline 235650 & 78.0431 & 148.2975 & 2421.99 & 1670 & 16 & 63172.1 \\
\hline 235950 & 78.0432 & 148.2846 & 2422.63 & 1672 & 16 & 0.0 \\
\hline 236100 & 78.0432 & 148.2781 & 2422.73 & 1673 & 16 & 0.0 \\
\hline 236250 & 78.0433 & 148.2716 & 2422.99 & 1674 & 16 & 63173.7 \\
\hline 236400 & 78.0433 & 148.2651 & 2423.08 & 1675 & 16 & 0.0 \\
\hline 236550 & 78.0433 & 148.2587 & 2423.39 & 1676 & 16 & 0.0 \\
\hline 236700 & 78.0434 & 148.2522 & 2423.53 & 1677 & 16 & 0.0 \\
\hline 236850 & 78.0434 & 148.2457 & 2423.67 & 1678 & 16 & 63181.8 \\
\hline 237000 & 78.0434 & 148.2392 & 2423.87 & 1679 & 16 & 63245.4 \\
\hline 237150 & 78.0435 & 148.2327 & 2424.24 & 1680 & 16 & 0.0 \\
\hline 237300 & 78.0435 & 148.2262 & 2424.24 & 1681 & 16 & 0.0 \\
\hline 237450 & 78.0435 & 148.2197 & 2424.46 & 1682 & 16 & 63308.0 \\
\hline 237600 & 78.0436 & 148.2133 & 2424.45 & 1683 & 16 & 0.0 \\
\hline 237750 & 78.0436 & 148.2068 & 2424.31 & 1684 & 16 & 0.0 \\
\hline 237900 & 78.0436 & 148.2003 & 2424.83 & 1685 & 16 & 0.0 \\
\hline 238050 & 78.0437 & 148.1938 & 2424.60 & 1686 & 16 & 63263.8 \\
\hline 238200 & 78.0437 & 148.1873 & 2424.45 & 1687 & 16 & 0.0 \\
\hline 238350 & 78.0437 & 148.1809 & 2424.78 & 1688 & 16 & 0.0 \\
\hline 238500 & 78.0437 & 148.1744 & 2424.88 & 1689 & 16 & 0.0 \\
\hline 238650 & 78.0438 & 148.1679 & 2424.78 & 1690 & 16 & 63245.5 \\
\hline 238800 & 78.0438 & 148.1614 & 2425.28 & 1691 & 16 & 0.0 \\
\hline 238950 & 78.0438 & 148.1550 & 2425.08 & 1692 & 16 & 0.0 \\
\hline 239100 & 78.0439 & 148.1484 & 2425.33 & 1693 & 16 & 63259.8 \\
\hline 239250 & 78.0439 & 148.1420 & 2425.22 & 1694 & 16 & 63254.5 \\
\hline 239400 & 78.0440 & 148.1355 & 2425.24 & 1695 & 16 & 0.0 \\
\hline 239550 & 78.0440 & 148.1290 & 2425.47 & 1696 & 16 & 0.0 \\
\hline 239700 & 78.0440 & 148.1225 & 2425.38 & 1697 & 16 & 0.0 \\
\hline 239850 & 78.0440 & 148.1160 & 2425.65 & 1698 & 16 & 63215.0 \\
\hline 240000 & 78.0441 & 148.1096 & 2425.75 & 1699 & 16 & 0.0 \\
\hline 240150 & 78.0441 & 148.1031 & 2425.40 & 1700 & 16 & 0.0 \\
\hline 240300 & 78.0441 & 148.0966 & 2425.87 & 1701 & 16 & 0.0 \\
\hline 240450 & 78.0442 & 148.0901 & 2425.58 & 1702 & 16 & 63218.9 \\
\hline 240600 & 78.0442 & 148.0836 & 2425.93 & 1703 & 16 & 0.0 \\
\hline 240750 & 78.0442 & 148.0772 & 2425.79 & 1704 & 16 & 0.0 \\
\hline 240900 & 78.0443 & 148.0707 & 2425.91 & 1705 & 16 & 0.0 \\
\hline 241050 & 78.0443 & 148.0642 & 2425.93 & 1706 & 16 & 63199.5 \\
\hline 241200 & 78.0443 & 148.0577 & 2426.11 & 1707 & 16 & 63223.6 \\
\hline 241350 & 78.0444 & 148.0318 & 2426.27 & 1708 & 16 & 0.0 \\
\hline 241500 & 78.0444 & 148.0447 & 2426.04 & 1709 & 16 & 0.0 \\
\hline 241650 & 78.0444 & 148.0382 & 2426.19 & 1710 & 20 & 63125 \\
\hline 241950 & 78.0444 & 148.0253 & 2426.55 & 1712 & 20 & 0.0 \\
\hline 242100 & 78.0444 & 148.0188 & 2426.55 & 1713 & 20 & 0.0 \\
\hline 242250 & 78.0444 & 148.0123 & 2426.61 & 1714 & 20 & 63153.2 \\
\hline 242400 & 78.0445 & 148.0058 & 2426.77 & 1715 & 20 & 0.0 \\
\hline 242550 & 78.0445 & 147.9994 & 2426.97 & 1716 & 20 & 0.0 \\
\hline 242700 & 78.0445 & 147.9929 & 2427.21 & 1717 & 20 & 0.0 \\
\hline 242850 & 78.0445 & 147.9864 & 2427.56 & 1718 & 20 & 63148.4 \\
\hline 243000 & 78.0445 & 147.9799 & 2427.44 & 1719 & 20 & 0.0 \\
\hline 243150 & 78.0445 & 147.9734 & 2427.49 & 1720 & 20 & 0.0 \\
\hline
\end{tabular}




\begin{tabular}{|c|c|c|c|c|c|c|}
\hline Distance $(\mathbf{m})$ & $\begin{array}{l}\text { Flag Loc. } \\
\left.\text { Latitude ( }{ }^{\circ} \mathbf{S}\right)\end{array}$ & $\begin{array}{l}\text { Flag Loc. } \\
\left.\text { Longitude ( }{ }^{\circ} \mathrm{E}\right)\end{array}$ & Elevation (m) & $\begin{array}{l}\text { Shot } \\
\text { Number }\end{array}$ & $\begin{array}{c}\text { Julian } \\
\text { Day }\end{array}$ & $\begin{array}{l}\text { Total Mag. } \\
\text { Field (nT) }\end{array}$ \\
\hline 243300 & 78.0445 & 147.9669 & 2427.58 & T721 & $\overline{20}$ & 63742.7 \\
\hline 243450 & 78.0446 & 147.9604 & 2427.61 & 1722 & 20 & 63177.8 \\
\hline 243600 & 78.0446 & 147.9539 & 2427.87 & 1723 & 20 & 0.0 \\
\hline 243750 & 78.0446 & 147.9475 & 2427.97 & 1724 & 20 & 0.0 \\
\hline 243900 & 78.0446 & 147.9410 & 2428.21 & 1725 & 20 & 0.0 \\
\hline 244050 & 78.0446 & 147.9345 & 2428.45 & 1726 & 20 & 63158.6 \\
\hline 244200 & 78.0446 & 147.9280 & 2428.61 & 1727 & 20 & 0.0 \\
\hline 244350 & 78.0446 & 147.9215 & 2428.92 & 1728 & 20 & 0.0 \\
\hline 244500 & 78.0447 & 147.9151 & 2429.11 & 1729 & 20 & 0.0 \\
\hline 244650 & 78.0447 & 147.9086 & 2429.16 & 1730 & 20 & 63185.4 \\
\hline 244800 & 78.0447 & 147.9021 & 2428.92 & 1731 & 20 & 0.0 \\
\hline 244950 & 78.0447 & 147.8956 & 2429.59 & 1732 & 20 & 0.0 \\
\hline 245100 & 78.0447 & 147.8891 & 2429.80 & 1733 & 20 & 0.0 \\
\hline 245250 & 78.0447 & 147.8827 & 2429.94 & 1734 & 20 & 63190.7 \\
\hline 245400 & 78.0447 & 147.8761 & 2430.13 & 1735 & 20 & 63201.5 \\
\hline 245550 & 78.0447 & 147.8697 & 2430.27 & 1736 & 20 & 0.0 \\
\hline 245700 & 78.0448 & 147.8632 & 2430.62 & 1737 & 20 & 0.0 \\
\hline 245850 & 78.0448 & 147.8567 & 2430.85 & 1738 & 20 & 63221.9 \\
\hline 246000 & 78.0448 & 147.8502 & 2431.13 & 1739 & 20 & 0.0 \\
\hline 246150 & 78.0448 & 147.8437 & 2431.24 & 1740 & 20 & 0.0 \\
\hline 246300 & 78.0448 & 147.8372 & 2431.34 & 1741 & 20 & 0.0 \\
\hline 246450 & 78.0448 & 147.8308 & 2431.53 & 1742 & 20 & 63217 \\
\hline 246600 & 78.0448 & 147.8243 & 2431.77 & 1743 & 20 & 0.0 \\
\hline 246750 & 78.0448 & 147.8178 & 2431.76 & 1744 & 20 & 0.0 \\
\hline 246900 & 78.0448 & 147.8113 & 2432.22 & 1745 & 20 & 0.0 \\
\hline 247050 & 78.0448 & 147.8048 & 2431.98 & 1746 & 20 & 63217.9 \\
\hline 247200 & 78.0448 & 147.7984 & 2432.01 & 1747 & 20 & 0.0 \\
\hline 247350 & 78.0448 & 147.7919 & 2432.26 & 1748 & 20 & 0.0 \\
\hline 247500 & 78.0448 & 147.7854 & 2432.67 & 1749 & 20 & 0.0 \\
\hline 247650 & 78.0448 & 147.7789 & 2432.71 & 1750 & 20 & 63220.6 \\
\hline 247800 & 78.0448 & 147.7724 & 2433.04 & 1751 & 20 & 63215 \\
\hline 247950 & 78.0448 & 147.7659 & 2433.24 & 1752 & 20 & 0.0 \\
\hline 248100 & 78.0449 & 147.7594 & 2433.26 & 1753 & 20 & 0.0 \\
\hline 248250 & 78.0449 & 147.7529 & 2433.13 & 1754 & 20 & 0.0 \\
\hline 248400 & 78.0449 & 147.7465 & 2433.31 & 1755 & 20 & 63238.4 \\
\hline 248550 & 78.0449 & 147.7400 & 2433.38 & 1756 & 20 & 0.0 \\
\hline 248700 & 78.0449 & 147.7335 & 2433.50 & 1757 & 20 & 0.0 \\
\hline 248850 & 78.0449 & 147.7270 & 2433.78 & 1758 & 20 & 0.0 \\
\hline 249000 & 78.0449 & 147.7205 & 2433.55 & 1759 & 20 & 63194.6 \\
\hline 249150 & 78.0449 & 147.7141 & 2433.66 & 1760 & 20 & 0.0 \\
\hline 249300 & 78.0449 & 147.7076 & 2433.91 & 1761 & 20 & 0.0 \\
\hline 249450 & 78.0449 & 147.7011 & 2433.85 & 1762 & 20 & 0.0 \\
\hline 249600 & 78.0449 & 147.6946 & 2433.71 & 1763 & 20 & 63238.1 \\
\hline 249750 & 78.0449 & 147.6881 & 2433.74 & 1764 & 20 & 0.0 \\
\hline 249900 & 78.0449 & 147.6816 & 2434.04 & 1765 & 20 & 63239.6 \\
\hline 250050 & 78.0449 & 147.6751 & 2434.00 & 1766 & 20 & 63248.9 \\
\hline 250200 & 78.0449 & 147.6686 & 2434.04 & 1767 & 20 & 0.0 \\
\hline 250350 & 78.0449 & 147.6622 & 2434.13 & 1768 & 20 & 0.0 \\
\hline 250500 & 78.0449 & 147.6557 & 2434.42 & 1769 & 20 & 0.0 \\
\hline 250650 & 78.0449 & 147.6492 & 2434.47 & 1770 & 20 & 63221 \\
\hline 250800 & 78.0449 & 147.6427 & 2434.21 & 1771 & 20 & 0.0 \\
\hline 250950 & 78.0449 & 147.6362 & 2434.51 & 1772 & 20 & 0.0 \\
\hline
\end{tabular}




\begin{tabular}{|c|c|c|c|c|c|c|}
\hline Distance $(m)$ & $\begin{array}{c}\text { Flag Loc. } \\
\text { Latitude ('S) }\end{array}$ & $\begin{array}{c}\text { Flag Loc. } \\
\text { Longitude }\left({ }^{\circ} \mathrm{E}\right)\end{array}$ & Elevation (m) & $\begin{array}{c}\text { Shot } \\
\text { Number }\end{array}$ & $\begin{array}{c}\text { Julian } \\
\text { Day }\end{array}$ & $\begin{array}{l}\text { Total Mag. } \\
\text { Field (nT) }\end{array}$ \\
\hline 251100 & 78.0449 & 147.6297 & $\overline{2434.5}$ & $\overline{7773}$ & $\overline{20}$ & 0 \\
\hline 251250 & 78.0449 & 147.6233 & 2434.55 & 1774 & 20 & 632257 \\
\hline 251400 & 78.0450 & 147.6168 & 2434.77 & 1775 & 20 & 0 \\
\hline 251550 & 78.0450 & 147.6103 & 2434.74 & 1776 & 20 & 0 \\
\hline 251700 & 78.0450 & 147.6038 & 2434.73 & 1777 & 20 & 0 \\
\hline 251850 & 78.0450 & 147.5973 & 2434.44 & 1778 & 20 & 632273 \\
\hline 252000 & 78.0450 & 147.5908 & 2435.04 & 1779 & 20 & 632283 \\
\hline 252150 & 78.0450 & 147.5843 & 2434.77 & 1780 & 20 & 0 \\
\hline 252300 & 78.0450 & 147.5779 & 2435.2 & 1781 & 20 & 0 \\
\hline 252450 & 78.0450 & 147.5714 & 2435 & 1782 & 20 & 632320 \\
\hline 252600 & 78.0450 & 147.5649 & 2435.22 & 1783 & 20 & 0 \\
\hline 252750 & 78.0450 & 147.5584 & 2435.33 & 1784 & 20 & 0 \\
\hline 252900 & 78.0450 & 147.5519 & 2435.29 & 1785 & & 0 \\
\hline 253050 & 78.0450 & 147.5454 & 2435.61 & 1786 & 20 & 63228.1 \\
\hline 253200 & 78.0450 & 147.5390 & 2435.62 & 1787 & 20 & 0.0 \\
\hline 253350 & 78.0450 & 147.5325 & 2435.71 & 1788 & 20 & 0.0 \\
\hline 253500 & 78.0450 & 147.5260 & 2435.78 & 1789 & 20 & 0.0 \\
\hline 253650 & 78.0450 & 147.5195 & 2435.96 & 1790 & 20 & 63220.4 \\
\hline 253800 & 78.0450 & 147.5130 & 2435.96 & 1791 & 20 & 0.0 \\
\hline 253950 & 78.0450 & 147.5066 & 2436.18 & 1792 & 20 & 0.0 \\
\hline 254100 & 78.0450 & 147.5000 & 2436.57 & 1793 & 20 & 63197.6 \\
\hline 254250 & 78.0450 & 147.4936 & 2436.81 & 1794 & 20 & 63198.7 \\
\hline 254400 & 78.0450 & 147.4871 & 2436.79 & 2102 & 20 & 0.0 \\
\hline 254550 & 78.0450 & 147.4806 & 2436.92 & 2101 & 20 & 0.0 \\
\hline 254700 & 78.0450 & 147.4741 & 2437.33 & 2100 & 20 & 0.0 \\
\hline 254850 & 78.0450 & 147.4676 & 2437.25 & 2099 & 20 & 0.0 \\
\hline 255000 & 78.0450 & 147.4612 & 2437.44 & 2098 & 20 & 0.0 \\
\hline 255150 & 78.0450 & 147.4547 & 2437.32 & 2097 & 23 & 63112 \\
\hline 255300 & 78.0450 & 147.4482 & 2437.73 & 2096 & 23 & 0.0 \\
\hline 255450 & 78.0450 & 147.4417 & 2437.57 & 2095 & 23 & 0.0 \\
\hline 255600 & 78.0450 & 147.4352 & 2437.91 & 2094 & 23 & 0.0 \\
\hline 255750 & 78.0450 & 147.4287 & 2437.77 & 2093 & 23 & 63113.4 \\
\hline 255900 & 78.0450 & 147.4223 & 2437.81 & 2092 & 23 & 0.0 \\
\hline 256050 & 78.0450 & 147.4158 & 2437.58 & 2091 & 23 & 0.0 \\
\hline 256200 & 78.0450 & 147.4093 & 2438.07 & 2090 & 23 & 63119.5 \\
\hline 256350 & 78.0450 & 147.4028 & 2438.02 & 2089 & 23 & 0.0 \\
\hline 256500 & 78.0450 & 147.3963 & 2438.14 & 2088 & 23 & 0.0 \\
\hline 256650 & 78.0450 & 147.3898 & 2438.32 & 2087 & 23 & 0.0 \\
\hline 256800 & 78.0450 & 147.3833 & 2438.31 & 2086 & 23 & 63120.1 \\
\hline 256950 & 78.0450 & 147.3768 & 2438.35 & 2085 & 23 & 0.0 \\
\hline 257100 & 78.0449 & 147.3704 & 2438.80 & 2084 & 23 & 0.0 \\
\hline 257250 & 78.0449 & 147.3639 & 2438.65 & 2083 & 23 & 0.0 \\
\hline 257400 & 78.0449 & 147.3574 & 2438.55 & 2082 & 23 & 63089.8 \\
\hline 257550 & 78.0449 & 147.3509 & 2438.49 & 2081 & 23 & 0.0 \\
\hline 257700 & 78.0449 & 147.3444 & 2438.61 & 2080 & 23 & 0.0 \\
\hline 257850 & 78.0449 & 147.3380 & 2438.59 & 2079 & 23 & 0.0 \\
\hline 258000 & 78.0449 & 147.3315 & 2438.74 & 2078 & 23 & 63080.9 \\
\hline 258150 & 78.0448 & 147.3250 & 2438.63 & 2077 & 23 & 0.0 \\
\hline 258300 & 78.0448 & 147.3185 & 2438.70 & 2076 & 23 & 63083.8 \\
\hline 258450 & 78.0448 & 147.3120 & 2438.44 & 2075 & 23 & 0.0 \\
\hline 258600 & 78.0448 & 147.3055 & 2438.75 & 2074 & 23 & 0.0 \\
\hline 258750 & 78.0448 & 147.2990 & 2438.72 & 2073 & 23 & 0.0 \\
\hline
\end{tabular}




\begin{tabular}{|c|c|c|c|c|c|c|}
\hline Distance $(\mathbf{m})$ & $\begin{array}{l}\text { Flag Loc. } \\
\left.\text { Latitude ( }{ }^{\circ} \mathbf{S}\right)\end{array}$ & $\begin{array}{c}\text { Flag Loc. } \\
\text { Longitude }\left({ }^{\circ} E\right)\end{array}$ & Elevation (m) & $\begin{array}{c}\text { Shot } \\
\text { Number }\end{array}$ & $\begin{array}{c}\text { Julian } \\
\text { Day }\end{array}$ & $\begin{array}{l}\text { Total Mag. } \\
\text { Field (nT) }\end{array}$ \\
\hline 258900 & 78.0448 & $\overline{147.2926}$ & 2438.63 & $\overline{2072}$ & $\overline{23}$ & 63700.1 \\
\hline 259050 & 78.0447 & 147.2861 & 2439.03 & 2071 & 23 & 0.0 \\
\hline 259200 & 78.0447 & 147.2796 & 2438.84 & 2070 & 23 & 0.0 \\
\hline 259350 & 78.0447 & 147.2731 & 2438.81 & 2069 & 23 & 0.0 \\
\hline 259500 & 78.0447 & 147.2666 & 2438.71 & 2068 & 23 & 63094.7 \\
\hline 259650 & 78.0447 & 147.2601 & 2438.73 & 2067 & 23 & 0.0 \\
\hline 259800 & 78.0447 & 147.2537 & 2438.72 & 2066 & 23 & 0.0 \\
\hline 259950 & 78.0446 & 147.2472 & 2438.74 & 2065 & 23 & 0.0 \\
\hline 260100 & 78.0446 & 147.2407 & 2439.07 & 2064 & 23 & 63104.7 \\
\hline 260250 & 78.0446 & 147.2342 & 2438.73 & 2063 & 23 & 0.0 \\
\hline 260400 & 78.0446 & 147.2277 & 2439.15 & 2062 & 23 & 63122.6 \\
\hline 260550 & 78.0446 & 147.2212 & 2439.25 & 2061 & 23 & 0.0 \\
\hline 260700 & 78.0446 & 147.2147 & 2439.20 & 2060 & 23 & 0.0 \\
\hline 260850 & 78.0445 & 147.2083 & 2439.30 & 2059 & 23 & 0.0 \\
\hline 261000 & 78.0444 & 147.1564 & 2440.17 & 2058 & 23 & 63119.5 \\
\hline 261300 & 78.0445 & 147.1888 & 2439.92 & 2056 & 23 & 0.0 \\
\hline 261450 & 78.0445 & 147.1823 & 2439.81 & 2055 & 23 & 0.0 \\
\hline 261600 & 78.0445 & 147.1759 & 2440.07 & 2054 & 23 & 63143.3 \\
\hline 261750 & 78.0444 & 147.1694 & 2440.35 & 2053 & 23 & 0.0 \\
\hline 261900 & 78.0444 & 147.1629 & 2440.53 & 2052 & 23 & 0.0 \\
\hline 262200 & 78.0444 & 147.1499 & 2440.45 & 2050 & 23 & 63133.2 \\
\hline 262350 & 78.0444 & 147.1434 & 2440.72 & 2049 & 23 & 0.0 \\
\hline 262500 & 78.0444 & 147.1369 & 2441.03 & 2048 & 23 & 63157.4 \\
\hline 262650 & 78.0443 & 147.1304 & 2441.24 & 2047 & 23 & 0.0 \\
\hline 262800 & 78.0443 & 147.1240 & 2441.65 & 2046 & 23 & 0.0 \\
\hline 262950 & 78.0443 & 147.1175 & 2441.93 & 2045 & 23 & 0.0 \\
\hline 263100 & 78.0443 & 147.1110 & 2442.17 & 2044 & 23 & 63152.6 \\
\hline 263250 & 78.0443 & 147.1045 & 2442.27 & 2043 & 23 & 0.0 \\
\hline 263400 & 78.0443 & 147.0981 & 2442.91 & 2042 & 23 & 0.0 \\
\hline 263550 & 78.0442 & 147.0916 & 2443.15 & 2041 & 23 & 0.0 \\
\hline 263700 & 78.0442 & 147.0851 & 2443.14 & 2040 & 23 & 63132.4 \\
\hline 263850 & 78.0442 & 147.0786 & 2443.61 & 2039 & 23 & 0.0 \\
\hline 264000 & 78.0442 & 147.0721 & 2444.13 & 2038 & 23 & 0.0 \\
\hline 264150 & 78.0442 & 147.0656 & 2444.43 & 2037 & 23 & 0.0 \\
\hline 264300 & 78.0442 & 147.0591 & 2445.11 & 2036 & 23 & 63118.8 \\
\hline 264450 & 78.0441 & 147.0527 & 2445.35 & 2035 & 23 & 0.0 \\
\hline 264600 & 78.0441 & 147.0462 & 2445.67 & 2034 & 23 & 0.0 \\
\hline 264750 & 78.0441 & 147.0397 & 2446.19 & 2033 & 23 & 0.0 \\
\hline 264900 & 78.0441 & 147.0332 & 2446.62 & 2032 & 23 & 63111.1 \\
\hline 265050 & 78.0441 & 147.0267 & 2446.87 & 2031 & 23 & 0.0 \\
\hline 265200 & 78.0440 & 147.0202 & 2447.38 & 2030 & 23 & 0.0 \\
\hline 265350 & 78.0440 & 147.0138 & 2447.78 & 2029 & 23 & 0 \\
\hline 265500 & 78.0440 & 147.0073 & 2448.44 & 2028 & 23 & 63141.1 \\
\hline 265650 & 78.0440 & 147.0008 & 2448.78 & 2027 & 23 & 0.0 \\
\hline 265800 & 78.0440 & 146.9943 & 2449.06 & 2026 & 23 & 0.0 \\
\hline 265950 & 78.0439 & 146.9879 & 2449.40 & 2025 & 23 & 0.0 \\
\hline 266100 & 78.0439 & 146.9813 & 2449.35 & 2024 & 23 & 63104.1 \\
\hline 266250 & 78.0439 & 146.9749 & 2449.86 & 2023 & 23 & 0.0 \\
\hline 266400 & 78.0439 & 146.9684 & 2450.45 & 2022 & 23 & 0.0 \\
\hline 266550 & 78.0439 & 146.9619 & 2450.51 & 2021 & 23 & 0.0 \\
\hline 266700 & 78.0439 & 146.9554 & 2450.64 & 2020 & 23 & 63107.4 \\
\hline 266850 & 78.0438 & 146.9489 & 2450.88 & 2019 & 23 & 0.0 \\
\hline
\end{tabular}




\begin{tabular}{|c|c|c|c|c|c|c|}
\hline Distance (m) & $\begin{array}{l}\text { Flag Loc. } \\
\text { Latitude ('s) }\end{array}$ & $\begin{array}{c}\text { Flag Loc. } \\
\text { Longitude }\left({ }^{\circ} \mathrm{E}\right)\end{array}$ & Elevation (m) & $\begin{array}{c}\text { Shot } \\
\text { Number }\end{array}$ & $\begin{array}{c}\text { Julian } \\
\text { Day }\end{array}$ & $\begin{array}{l}\text { Total Mag. } \\
\text { Field (nT) }\end{array}$ \\
\hline$\overline{267000}$ & 78.0438 & $\longdiv { 7 4 6 . 9 4 2 5 }$ & 2450.99 & 2018 & $\overline{23}$ & 0.0 \\
\hline 267150 & 78.0438 & 146.9360 & 2451.40 & 2017 & 23 & 0.0 \\
\hline 267300 & 78.0438 & 146.9295 & 2451.27 & 2016 & 23 & 63112.4 \\
\hline 267450 & 78.0438 & 146.9230 & 2451.26 & 2015 & 23 & 0.0 \\
\hline 267600 & 78.0437 & 146.9165 & 2451.83 & 2014 & 23 & 0.0 \\
\hline 267750 & 78.0437 & 146.9100 & 2452.11 & 2013 & 23 & 0.0 \\
\hline 267900 & 78.0437 & 146.9036 & 2452.08 & 2012 & 23 & 63121.5 \\
\hline 268050 & 78.0437 & 146.8971 & 2452.13 & 2011 & 23 & 0.0 \\
\hline 268200 & 78.0437 & 146.8906 & 2452.15 & 2010 & 23 & 63124.9 \\
\hline 268350 & 78.0436 & 146.8841 & 2452.55 & 2009 & 23 & 0.0 \\
\hline 268500 & 78.0436 & 146.8776 & 2452.68 & 2008 & 23 & 0.0 \\
\hline 268650 & 78.0436 & 146.8711 & 2452.76 & 2007 & 23 & 0.0 \\
\hline 268800 & 78.0436 & 146.8647 & 2452.48 & 2006 & 23 & 63128 \\
\hline 268950 & 78.0436 & 146.8582 & 2452.71 & 2005 & 23 & 0.0 \\
\hline 269100 & 78.0435 & 146.8517 & 2452.97 & 2004 & 23 & 0.0 \\
\hline 269250 & 78.0435 & 146.8452 & 2452.81 & 2003 & 23 & 0.0 \\
\hline 269400 & 78.0435 & 146.8387 & 2452.94 & 2002 & 23 & 63143 \\
\hline 269550 & 78.0435 & 146.8322 & 2453.08 & 2001 & 23 & 0.0 \\
\hline 269700 & 78.0434 & 146.8258 & 2453.12 & 이 & 23 & 0.0 \\
\hline 269850 & 78.0434 & 146.8193 & 2453.05 & wo4001 & 23 & 0.0 \\
\hline 270000 & 78.0434 & 146.8128 & 2452.84 & 이 & 23 & 63123.4 \\
\hline 270150 & 78.0434 & 146.8063 & 2453.19 & wo4002 & 23 & 0.0 \\
\hline 270300 & 78.0434 & 146.7998 & 2453.52 & 0 & 24 & 63217.6 \\
\hline 270450 & 78.0434 & 146.7934 & 2453.61 & wo4003 & 24 & 0.0 \\
\hline 270600 & 78.0433 & 146.7869 & 2453.44 & o & 24 & 0.0 \\
\hline 270750 & 78.0433 & 146.7804 & 2453.83 & wo4004 & 24 & 0.0 \\
\hline 270900 & 78.0433 & 146.7739 & 2454.11 & ol & 24 & 0.0 \\
\hline 271050 & 78.0433 & 146.7674 & 2454.08 & wo4005 & 24 & 63211.2 \\
\hline 271350 & 78.0432 & 146.7545 & 2454.68 & 이 & 24 & 0.0 \\
\hline 271500 & 78.0432 & 146.7480 & 2454.51 & wo4006 & 24 & 0.0 \\
\hline 271650 & 78.0432 & 146.7415 & 2454.64 & 이 & 24 & 63214.1 \\
\hline 271800 & 78.0432 & 146.7350 & 2454.79 & w04007| & 24 & 0.0 \\
\hline 271950 & 78.0431 & 146.7285 & 2454.79 & 이 & 24 & 0.0 \\
\hline 272100 & 78.0431 & 146.7221 & 2455.01 & wo4008 & 24 & 0.0 \\
\hline 272250 & 78.0431 & 146.7156 & 2455.07 & 0 & 24 & 0.0 \\
\hline 272400 & 78.0431 & 146.7091 & 2455.66 & wo4009 & 24 & 63222.5 \\
\hline 272550 & 78.0430 & 146.7026 & 2455.55 & 이 & 24 & 0.0 \\
\hline 272700 & 78.0430 & 146.6961 & 2455.63 & wo4010 & 24 & 0.0 \\
\hline 272850 & 78.0430 & 146.6896 & 2455.79 & o & 24 & 0.0 \\
\hline 273000 & 78.0430 & 146.6832 & 2456.16 & o & 24 & 0.0 \\
\hline 273150 & 78.0429 & 146.6767 & 2456.30 & 0 & 24 & 63232.2 \\
\hline 273300 & 78.0429 & 146.6702 & 2456.27 & 이 & 24 & 0.0 \\
\hline 273450 & 78.0429 & 146.6637 & 2456.53 & ol & 24 & 0.0 \\
\hline 273600 & 78.0429 & 146.6572 & 2456.65 & 이 & 24 & 0.0 \\
\hline 273750 & 78.0428 & 146.6508 & 2456.63 & 이 & 24 & 0.0 \\
\hline 273900 & 78.0428 & 146.6443 & 2456.85 & 이 & 24 & 63222.5 \\
\hline 274050 & 78.0428 & 146.6378 & 2456.69 & 이 & 24 & 0.0 \\
\hline 274200 & 78.0428 & 146.6313 & 2456.86 & ol & 24 & 0.0 \\
\hline 274350 & 78.0427 & 146.6248 & 2456.71 & o & 24 & 0.0 \\
\hline 274500 & 78.0427 & 146.6183 & 2457.15 & o & 24 & 63243.7 \\
\hline 274650 & 78.0427 & 146.6118 & 2457.24 & o & 24 & 0.0 \\
\hline 274800 & 78.0427 & 146.6054 & 2457.31 & ol & 24 & 0.0 \\
\hline
\end{tabular}




\begin{tabular}{|c|c|c|c|c|c|c|}
\hline Distance $(\mathbf{m})$ & $\begin{array}{l}\text { Flag Loc. } \\
\left.\text { Latitude ( }{ }^{\circ} \mathbf{S}\right)\end{array}$ & $\begin{array}{c}\text { Flag Loc. } \\
\text { Longitude }\left({ }^{\circ} \mathrm{E}\right)\end{array}$ & Elevation (m) & $\begin{array}{c}\text { Shot } \\
\text { Number }\end{array}$ & $\begin{array}{c}\text { Julian } \\
\text { Day }\end{array}$ & $\begin{array}{l}\text { Total Mag. } \\
\text { Field (nT) }\end{array}$ \\
\hline 274950 & 78.0426 & $\longdiv { 1 4 6 . 5 9 8 9 }$ & 2457.21 & 0 & 24 & 0.0 \\
\hline 275100 & 78.0426 & 146.5924 & 2457.20 & 0 & 24 & 0.0 \\
\hline 275250 & 78.0426 & 146.5859 & 2457.13 & 0 & 24 & 63234.8 \\
\hline 275400 & 78.0426 & 146.5795 & 2456.98 & 0 & 24 & 0.0 \\
\hline 275550 & 78.0425 & 146.5730 & 2456.93 & 0 & 24 & 0.0 \\
\hline 275700 & 78.0425 & 146.5665 & 2457.14 & 0 & 24 & 0.0 \\
\hline 275850 & 78.0425 & 146.5600 & 2457.04 & 0 & 24 & 0.0 \\
\hline 276000 & 78.0425 & 146.5535 & 2457.10 & 0 & 24 & 63246.6 \\
\hline 276150 & 78.0424 & 146.5470 & 2456.91 & 0 & 24 & 0.0 \\
\hline 276300 & 78.0424 & 146.5406 & 2456.83 & 0 & 24 & 0.0 \\
\hline 276450 & 78.0424 & 146.5341 & 2457.28 & 0 & 24 & 0.0 \\
\hline 276600 & 78.0424 & 146.5276 & 2457.23 & 0 & 24 & 63257.2 \\
\hline 276750 & 78.0423 & 146.5211 & 2457.36 & 0 & 24 & 0.0 \\
\hline 276900 & 78.0423 & 146.5146 & 2457.27 & 0 & 24 & 0.0 \\
\hline 277050 & 78.0423 & 146.5081 & 2457.41 & 0 & 24 & 0.0 \\
\hline 277200 & 78.0423 & 146.5017 & 2457.45 & 0 & 24 & 0.0 \\
\hline 277350 & 78.0422 & 146.4952 & 2457.53 & 0 & 24 & 63245.3 \\
\hline 277500 & 78.0422 & 146.4887 & 2457.50 & 0 & 24 & 0.0 \\
\hline 277650 & 78.0422 & 146.4822 & 2457.59 & 0 & 24 & 0.0 \\
\hline 277800 & 78.0422 & 146.4757 & 2457.97 & 0 & 24 & 0.0 \\
\hline 277950 & 78.0421 & 146.4693 & 2458.03 & 0 & 24 & 0.0 \\
\hline 278100 & 78.0421 & 146.4628 & 2458.09 & 0 & 24 & 63249.9 \\
\hline 278250 & 78.0421 & 146.4563 & 2458.37 & 0 & 24 & 0.0 \\
\hline 278400 & 78.0420 & 146.4498 & 2458.76 & 0 & 24 & 0.0 \\
\hline 278550 & 78.0420 & 146.4433 & 2458.79 & 0 & 24 & 0.0 \\
\hline 278700 & 78.0420 & 146.4368 & 2459.14 & 0 & 24 & 63242.5 \\
\hline 278850 & 78.0420 & 146.4303 & 2459.08 & 0 & 24 & 0.0 \\
\hline 279000 & 78.0419 & 146.4239 & 2459.19 & 0 & 24 & 0.0 \\
\hline 279150 & 78.0419 & 146.4174 & 2459.58 & 0 & 24 & 0.0 \\
\hline 279300 & 78.0419 & 146.4109 & 2459.59 & 이 & 24 & 0.0 \\
\hline 279450 & 78.0419 & 146.4044 & 2459.81 & 0] & 24 & 63247.5 \\
\hline 279600 & 78.0418 & 146.3980 & 2459.90 & 0 & 24 & 0.0 \\
\hline 279750 & 78.0418 & 146.3915 & 2460.35 & 0 & 24 & 0.0 \\
\hline 279900 & 78.0418 & 146.3850 & 2460.43 & 0 & 24 & 0.0 \\
\hline 280050 & 78.0417 & 146.3785 & 2460.66 & 0 & 24 & 0.0 \\
\hline 280200 & 78.0417 & 146.3720 & 2460.74 & o & 24 & 63238.1 \\
\hline 280350 & 78.0417 & 146.3656 & 2460.68 & 0 & 24 & 0.0 \\
\hline 280500 & 78.0417 & 146.3591 & 2461.14 & o & 24 & 0.0 \\
\hline 280650 & 78.0416 & 146.3526 & 2461.27 & 0 & 24 & 0.0 \\
\hline 280800 & 78.0416 & 146.3461 & 2461.27 & 0 & 24 & 63240 \\
\hline 280950 & 78.0416 & 146.3396 & 2461.50 & 0 & 24 & 0.0 \\
\hline 281400 & 78.0415 & 146.3202 & 2462.18 & 0 & 24 & 0.0 \\
\hline 281550 & 78.0415 & 146.3137 & 2462.22 & 0 & 24 & 63238.6 \\
\hline 281700 & 78.0414 & 146.3072 & 2462.16 & 이 & 24 & 0.0 \\
\hline 281850 & 78.0414 & 146.3007 & 2462.22 & o) & 24 & 0.0 \\
\hline 282000 & 78.0414 & 146.2943 & 2462.88 & 이 & 24 & 0.0 \\
\hline 282150 & 78.0414 & 146.2878 & 2463.00 & 이 & 24 & 0.0 \\
\hline 282300 & 78.0413 & 146.2813 & 2462.76 & 0 & 24 & 63220.6 \\
\hline 282450 & 78.0413 & 146.2748 & 2462.96 & 0 & 24 & 0.0 \\
\hline 282600 & 78.0413 & 146.2684 & 2463.30 & 0 & 24 & 0.0 \\
\hline 282750 & 78.0412 & 146.2619 & 2463.51 & 0 & 24 & 0.0 \\
\hline 282900 & 78.0412 & 146.2554 & 2463.68 & 0 & 24 & 63231.6 \\
\hline
\end{tabular}




\begin{tabular}{|c|c|c|c|c|c|c|}
\hline Distance (m) & $\begin{array}{l}\text { Flag Loc. } \\
\left.\text { Latitude ( }{ }^{\circ} \mathrm{S}\right)\end{array}$ & $\begin{array}{c}\text { Flag Loc. } \\
\text { Longitude }\left({ }^{\circ} \mathrm{E}\right)\end{array}$ & Elevation (m) & $\begin{array}{c}\text { Shot } \\
\text { Number }\end{array}$ & $\begin{array}{c}\text { Julian } \\
\text { Day }\end{array}$ & $\begin{array}{l}\text { Total Mag. } \\
\text { Field (nT) }\end{array}$ \\
\hline 283050 & 78.0412 & 746.2489 & 2463.62 & $\overline{0}$ & $\overline{24}$ & $\overline{0.0}$ \\
\hline 283200 & 78.0411 & 146.2424 & 2463.78 & 0 & 24 & 0.0 \\
\hline 283350 & 78.0411 & 146.2359 & 2463.91 & 0 & 24 & 0.0 \\
\hline 283500 & 78.0410 & 146.2295 & 2463.90 & 0 & 24 & 0.0 \\
\hline 283650 & 78.0409 & 146.2230 & 2464.37 & 0 & 24 & 63218.8 \\
\hline 283800 & 78.0409 & 146.2165 & 2464.43 & 0 & 24 & 0.0 \\
\hline 283950 & 78.0408 & 146.2100 & 2464.78 & 0 & 24 & 0.0 \\
\hline 284100 & 78.0408 & 146.2036 & 2464.46 & 0 & 24 & 0.0 \\
\hline 284250 & 78.0407 & 146.1971 & 2464.49 & o & 24 & 0.0 \\
\hline 284400 & 78.0407 & 146.1906 & 2464.69 & ol & 24 & 63202 \\
\hline 284550 & 78.0406 & 146.1841 & 2464.65 & 0 & 24 & 0.0 \\
\hline 284700 & 78.0405 & 146.1777 & 2464.80 & o & 24 & 0.0 \\
\hline 284850 & 78.0405 & 146.1712 & 2464.72 & 0 & 24 & 0.0 \\
\hline 285000 & 78.0404 & 146.1647 & 2464.97 & 0 & 24 & 63205.4 \\
\hline 285150 & 78.0404 & 146.1582 & 2465.33 & 이 & 24 & 0.0 \\
\hline 285300 & 78.0403 & 146.1517 & 2465.00 & o & 24 & 0.0 \\
\hline 285450 & 78.0403 & 146.1453 & 2464.77 & 0 & 24 & 0.0 \\
\hline 285600 & 78.0402 & 146.1388 & 2465.02 & 이 & 24 & 0.0 \\
\hline 285750 & 78.0401 & 146.1323 & 2464.82 & 이 & 24 & 63192 \\
\hline 285900 & 78.0401 & 146.1258 & 2464.77 & 이 & 24 & 0.0 \\
\hline 286050 & 78.0400 & 146.1194 & 2464.66 & 이 & 24 & 0.0 \\
\hline 286200 & 78.0400 & 146.1129 & 2464.74 & 이 & 24 & 0.0 \\
\hline 286350 & 78.0399 & 146.1064 & 2464.71 & 0 & 24 & 0.0 \\
\hline 286500 & 78.0398 & 146.1000 & 2464.35 & 이 & 24 & 0.0 \\
\hline 286650 & 78.0398 & 146.0935 & 2464.34 & 이 & 24 & 63189.9 \\
\hline 286800 & 78.0397 & 146.0870 & 2463.82 & 이 & 24 & 0.0 \\
\hline 286950 & 78.0397 & 146.0805 & 2463.80 & 이 & 24 & 0.0 \\
\hline 287100 & 78.0396 & 146.0740 & 2464.12 & 이 & 24 & 63169.4 \\
\hline 287250 & 78.0396 & 146.0676 & 2463.60 & 0 & 24 & 0.0 \\
\hline 287400 & 78.0395 & 146.0611 & 2463.60 & 이 & 24 & 0.0 \\
\hline 287550 & 78.0394 & 146.0546 & 2463.43 & 0 & 24 & 0.0 \\
\hline 287700 & 78.0394 & 146.0481 & 2463.27 & of & 24 & 0.0 \\
\hline 287850 & 78.0393 & 146.0417 & 2463.17 & 이 & 24 & 63176.8 \\
\hline 288000 & 78.0393 & 146.0352 & 2463.07 & 이 & 24 & 0.0 \\
\hline 288150 & 78.0392 & 146.0287 & 2463.06 & 이 & 24 & 0.0 \\
\hline 288300 & 78.0391 & 146.0222 & 2462.78 & 이 & 24 & 0.0 \\
\hline 288450 & 78.0391 & 146.0158 & 2462.46 & 이 & 24 & 0.0 \\
\hline 288600 & 78.0390 & 146.0093 & 2462.53 & 이 & 24 & 63187.9 \\
\hline 288750 & 78.0390 & 146.0028 & 2462.45 & 이 & 24 & 0.0 \\
\hline 288900 & 78.0389 & 145.9964 & 2462.31 & 이 & 24 & 0.0 \\
\hline 289050 & 78.0388 & 145.9899 & 2462.22 & 이 & 24 & 0.0 \\
\hline 289200 & 78.0388 & 145.9834 & 2462.29 & 이 & 24 & 63184.1 \\
\hline 289350 & 78.0387 & 145.9769 & 2462.40 & 이 & 24 & 0.0 \\
\hline 289500 & 78.0387 & 145.9704 & 2462.45 & 이 & 24 & 0.0 \\
\hline 289650 & 78.0386 & 145.9640 & 2462.43 & 이 & 24 & 0.0 \\
\hline 289800 & 78.0386 & 145.9575 & 2462.39 & 이 & 24 & 0.0 \\
\hline 289950 & 78.0385 & 145.9510 & 2462.35 & 이 & 24 & 63195.8 \\
\hline 290100 & 78.0384 & 145.9445 & 2462.51 & 이 & 24 & 0.0 \\
\hline 290250 & 78.0384 & 145.9381 & 2462.40 & 이 & 24 & 0.0 \\
\hline 290400 & 78.0383 & 145.9316 & 2462.66 & 이 & 24 & 0.0 \\
\hline 290550 & 78.0383 & 145.9251 & 2462.46 & 이 & 24 & 0.0 \\
\hline 290700 & 78.0382 & 145.9187 & 2462.50 & 이 & 24 & 63185.6 \\
\hline
\end{tabular}




\begin{tabular}{|c|c|c|c|c|c|c|}
\hline Distance $(\mathbf{m})$ & $\begin{array}{l}\text { Flag Loc. } \\
\left.\text { Latitude ( }{ }^{\circ} \mathrm{S}\right)\end{array}$ & $\begin{array}{c}\text { Flag Loc. } \\
\left.\text { Longitude ( }{ }^{\circ} \mathrm{E}\right)\end{array}$ & Elevation (m) & $\begin{array}{l}\text { Shot } \\
\text { Number }\end{array}$ & $\begin{array}{l}\text { Julian } \\
\text { Day }\end{array}$ & $\begin{array}{l}\text { Total Mag. } \\
\text { Field }(\mathrm{nT})\end{array}$ \\
\hline 290850 & 78.0381 & 145.9122 & 2462.60 & $\overline{0}$ & $\overline{24}$ & $\overline{0.0}$ \\
\hline 291000 & 78.0381 & 145.9057 & 2462.74 & 0 & 24 & 0.0 \\
\hline 291150 & 78.0380 & 145.8992 & 2462.54 & 0 & 24 & 0.0 \\
\hline 291300 & 78.0379 & 145.8927 & 2463.13 & 0 & 24 & 63196 \\
\hline 291450 & 78.0379 & 145.8863 & 2463.20 & 0 & 24 & 0.0 \\
\hline 291600 & 78.0378 & 145.8798 & 2463.11 & 0 & 24 & 0.0 \\
\hline 291750 & 78.0378 & 145.8733 & 2463.33 & 0 & 24 & 0.0 \\
\hline 291900 & 78.0377 & 145.8669 & 2463.32 & 0 & 24 & 0.0 \\
\hline 292050 & 78.0376 & 145.8604 & 2463.52 & 0 & 24 & 63190.7 \\
\hline 292200 & 78.0376 & 145.8539 & 2463.40 & 0 & 24 & 0.0 \\
\hline 292350 & 78.0375 & 145.8474 & 2463.67 & 0 & 24 & 0.0 \\
\hline 292500 & 78.0375 & 145.8410 & 2463.72 & 0 & 24 & 0.0 \\
\hline 292650 & 78.0374 & 145.8345 & 2463.63 & 0 & 24 & 0.0 \\
\hline 292800 & 78.0373 & 145.8280 & 2463.76 & 0 & 24 & 0.0 \\
\hline 292950 & 78.0373 & 145.8215 & 2463.80 & 0 & 24 & 63218.3 \\
\hline 293100 & 78.0372 & 145.8151 & 2464.02 & 0 & 24 & 0.0 \\
\hline 293250 & 78.0371 & 145.8086 & 2463.72 & 0 & 24 & 0.0 \\
\hline 293400 & 78.0371 & 145.8021 & 2464.30 & 0 & 24 & 63207.1 \\
\hline 293550 & 78.0370 & 145.7956 & 2464.56 & 0 & 24 & 0.0 \\
\hline 293700 & 78.0370 & 145.7892 & 2464.35 & 0 & 24 & 0.0 \\
\hline 293850 & 78.0369 & 145.7827 & 2464.58 & 0 & 24 & 0.0 \\
\hline 294000 & 78.0368 & 145.7762 & 2464.31 & 0 & 24 & 0.0 \\
\hline 294150 & 78.0368 & 145.7697 & 2464.53 & 0 & 24 & 63221.4 \\
\hline 294300 & 78.0367 & 145.7633 & 2464.46 & 0 & 24 & 0.0 \\
\hline 294450 & 78.0366 & 145.7568 & 2464.36 & 0 & 24 & 0.0 \\
\hline 294600 & 78.0366 & 145.7503 & 2464.34 & 0 & 24 & 0.0 \\
\hline 294750 & 78.0365 & 145.7439 & 2464.71 & 0 & 24 & 0.0 \\
\hline 294900 & 78.0364 & 145.7374 & 2464.45 & 0 & 24 & 63240.1 \\
\hline 295050 & 78.0364 & 145.7309 & 2464.34 & 0 & 24 & 0.0 \\
\hline 295200 & 78.0363 & 145.7245 & 2464.45 & 0 & 24 & 0.0 \\
\hline 295350 & 78.0362 & 145.7180 & 2464.52 & 0 & 24 & 0.0 \\
\hline 295500 & 78.0362 & 145.7115 & 2464.95 & 0 & 24 & 63249.5 \\
\hline 295650 & 78.0361 & 145.7050 & 2464.94 & 0 & 24 & 0.0 \\
\hline 295800 & 78.0361 & 145.6985 & 2465.20 & 0 & 24 & 0.0 \\
\hline 295950 & 78.0360 & 145.6921 & 2465.02 & 0 & 24 & 0.0 \\
\hline 296100 & 78.0359 & 145.6856 & 2465.05 & 0 & 24 & 0.0 \\
\hline 296250 & 78.0359 & 145.6791 & 2465.09 & 0 & 25 & 63252 \\
\hline 296400 & 78.0358 & 145.6727 & 2465.15 & 0 & 25 & 0.0 \\
\hline 296550 & 78.0357 & 145.6662 & 2465.08 & 0 & 25 & 0.0 \\
\hline 296700 & 78.0357 & 145.6597 & 2465.17 & 0 & 25 & 0.0 \\
\hline 296850 & 78.0356 & 145.6533 & 2465.03 & 0 & 25 & 0.0 \\
\hline 297000 & 78.0355 & 145.6468 & 2464.66 & 0 & 25 & 63268.4 \\
\hline 297150 & 78.0355 & 145.6403 & 2464.71 & 0 & 25 & 0.0 \\
\hline 297300 & 78.0354 & 145.6339 & 2464.60 & 0 & 25 & 0.0 \\
\hline 297450 & 78.0353 & 145.6274 & 2464.58 & 0 & 25 & 0.0 \\
\hline 297600 & 78.0353 & 145.6209 & 2464.59 & 0 & 25 & 63260.1 \\
\hline 297750 & 78.0352 & 145.6144 & 2464.51 & 0 & 25 & 0.0 \\
\hline 297900 & 78.0351 & 145.6079 & 2464.07 & 0 & 25 & 0.0 \\
\hline 298050 & 78.0351 & 145.6015 & 2463.94 & 0 & 25 & 0.0 \\
\hline 298200 & 78.0350 & 145.5950 & 2463.63 & 0 & 25 & 0.0 \\
\hline 298350 & 78.0349 & 145.5885 & 2463.52 & 0 & 25 & 0.0 \\
\hline 298500 & 78.0349 & 145.5821 & 2463.37 & 0 & 25 & 63279. \\
\hline
\end{tabular}




\begin{tabular}{|c|c|c|c|c|c|c|}
\hline Distance $(\mathrm{m})$ & $\begin{array}{c}\text { Flag Loc. } \\
\text { Latitude ('S) }\end{array}$ & $\begin{array}{c}\text { Flag Loc. } \\
\text { Longitude }\left({ }^{\circ} \mathrm{E}\right)\end{array}$ & Elevation (m) & $\begin{array}{c}\text { Shot } \\
\text { Number }\end{array}$ & $\begin{array}{l}\text { Julian } \\
\text { Day }\end{array}$ & $\begin{array}{l}\text { Total Mag. } \\
\text { Field (nT) }\end{array}$ \\
\hline 298650 & 78.0348 & $\overline{145.5756}$ & 2463.21 & $\overline{\overline{0}}$ & $\overline{25}$ & $\overline{0.0}$ \\
\hline 298800 & 78.0347 & 145.5691 & 2462.96 & 0 & 25 & 0.0 \\
\hline 298950 & 78.0347 & 145.5627 & 2462.77 & 0 & 25 & 0.0 \\
\hline 299100 & 78.0346 & 145.5562 & 2462.45 & 0 & 25 & 0.0 \\
\hline 299250 & 78.0345 & 145.5497 & 2462.36 & 0 & 25 & 63293.3 \\
\hline 299400 & 78.0345 & 145.5433 & 2462.48 & 0 & 25 & 0.0 \\
\hline 299550 & 78.0344 & 145.5368 & 2461.98 & 0 & 25 & 0.0 \\
\hline 299700 & 78.0343 & 145.5303 & 2462.26 & 0 & 25 & 63290.4 \\
\hline 299850 & 78.0342 & 145.5238 & 2462.34 & 0 & 25 & 0.0 \\
\hline 300000 & 78.0342 & 145.5173 & 2462.00 & 0 & 25 & 0.0 \\
\hline 300150 & 78.0341 & 145.5109 & 2462.04 & 0 & 25 & 0.0 \\
\hline 300300 & 78.0340 & 145.5044 & 2462.30 & 0 & 25 & 0.0 \\
\hline 300450 & 78.0340 & 145.4980 & 2462.15 & 0 & 25 & 63288.3 \\
\hline 300600 & 78.0339 & 145.4915 & 2462.46 & 0 & 25 & 0.0 \\
\hline 300750 & 78.0338 & 145.4850 & 2462.49 & 0 & 25 & 0.0 \\
\hline 300900 & 78.0338 & 145.4785 & 2462.27 & 0 & 25 & 0.0 \\
\hline 301050 & 78.0337 & 145.4721 & 2462.61 & 0 & 25 & 0.0 \\
\hline 301200 & 78.0336 & 145.4656 & 2462.63 & 0 & 25 & 63284.4 \\
\hline 301350 & 78.0336 & 145.4592 & 2462.81 & 0 & 25 & 0.0 \\
\hline 301500 & 78.0335 & 145.4527 & 2462.80 & 0 & 25 & 0.0 \\
\hline 301650 & 78.0334 & 145.4462 & 2462.99 & 0 & 25 & 0.0 \\
\hline 301800 & 78.0333 & 145.4397 & 2463.35 & 0 & 25 & 63280 \\
\hline 301950 & 78.0333 & 145.4332 & 2463.52 & 0 & 25 & 0.0 \\
\hline 302100 & 78.0332 & 145.4268 & 2463.57 & 0 & 25 & 0.0 \\
\hline 302250 & 78.0331 & 145.4203 & 2463.86 & 0 & 25 & 0.0 \\
\hline 302400 & 78.0331 & 145.4138 & 2464.17 & 0 & 25 & 0.0 \\
\hline 302550 & 78.0330 & 145.4074 & 2464.11 & 0 & 25 & 63297 \\
\hline 302700 & 78.0329 & 145.4009 & 2464.44 & 0 & 25 & 0.0 \\
\hline 302850 & 78.0328 & 145.3944 & 2464.47 & 0 & 25 & 0.0 \\
\hline 303000 & 78.0328 & 145.3880 & 2464.63 & 0 & 25 & 0.0 \\
\hline 303150 & 78.0327 & 145.3815 & 2465.02 & 0 & 25 & 0.0 \\
\hline 303300 & 78.0326 & 145.3750 & 2465.18 & 0 & 25 & 63303.9 \\
\hline 303450 & 78.0326 & 145.3686 & 2465.34 & 0 & 25 & 0.0 \\
\hline 303600 & 78.0325 & 145.3621 & 2465.50 & 0 & 25 & 0.0 \\
\hline 303750 & 78.0324 & 145.3557 & 2465.64 & 0 & 25 & 0.0 \\
\hline 303900 & 78.0323 & 145.3492 & 2466.22 & 0 & 25 & 63310.8 \\
\hline 304050 & 78.0323 & 145.3427 & 2465.98 & 0 & 25 & 0.0 \\
\hline 304200 & 78.0322 & 145.3362 & 2466.35 & 0 & 25 & 0.0 \\
\hline 304350 & 78.0321 & 145.3297 & 2466.58 & 0 & 25 & 0.0 \\
\hline 304500 & 78.0321 & 145.3233 & 2466.65 & 0 & 25 & 0.0 \\
\hline 304650 & 78.0320 & 145.3168 & 2466.85 & 0 & 25 & 63326.1 \\
\hline 304800 & 78.0319 & 145.3103 & 2467.32 & 0 & 25 & 0.0 \\
\hline 304950 & 78.0318 & 145.3039 & 2467.39 & 0 & 25 & 0.0 \\
\hline 305100 & 78.0318 & 145.2974 & 2467.67 & 0 & 25 & 0.0 \\
\hline 305250 & 78.0317 & 145.2910 & 2468.09 & 0 & 25 & 0.0 \\
\hline 305400 & 78.0316 & 145.2845 & 2468.15 & 0 & 25 & 63332.2 \\
\hline 305550 & 78.0315 & 145.2780 & 2468.34 & 0 & 25 & 0.0 \\
\hline 305700 & 78.0315 & 145.2716 & 2468.69 & 0 & 25 & 0.0 \\
\hline 305850 & 78.0314 & 145.2651 & 2469.02 & 0 & 25 & 0.0 \\
\hline 306000 & 78.0313 & 145.2586 & 2469.19 & 0 & 25 & 63327.4 \\
\hline 306150 & 78.0312 & 145.2521 & 2469.31 & 0 & 25 & 0.0 \\
\hline 306300 & 78.0312 & 145.2457 & 2469.67 & 0 & 25 & 0.0 \\
\hline
\end{tabular}




\begin{tabular}{|c|c|c|c|c|c|c|}
\hline Distance $(m)$ & $\begin{array}{c}\text { Flag Loc. } \\
\left.\text { Latitude ( }{ }^{\circ} \mathrm{S}\right)\end{array}$ & $\begin{array}{c}\text { Flag Loc. } \\
\text { Longitude }\left({ }^{\circ} \mathrm{E}\right)\end{array}$ & Elevation (m) & $\begin{array}{c}\text { Shot } \\
\text { Number }\end{array}$ & $\begin{array}{c}\text { Julian } \\
\text { Day }\end{array}$ & $\begin{array}{l}\text { Total Mag. } \\
\text { Field (nT) }\end{array}$ \\
\hline 306450 & 78.0377 & $74 \overline{145392}$ & 2469.93 & $\overline{0}$ & $\overline{25}$ & $\overline{0.0}$ \\
\hline 306600 & 78.0310 & 145.2327 & 2470.24 & 0 & 25 & 0.0 \\
\hline 306750 & 78.0310 & 145.2263 & 2470.19 & 0 & 25 & 63346.2 \\
\hline 306900 & 78.0309 & 145.2198 & 2470.42 & 0 & 25 & 0.0 \\
\hline 307050 & 78.0308 & 145.2133 & 2470.60 & 0 & 25 & 0.0 \\
\hline 307200 & 78.0307 & 145.2069 & 2470.86 & 0 & 25 & 0.0 \\
\hline 307350 & 78.0306 & 145.2004 & 2470.91 & 0 & 25 & 0.0 \\
\hline 307500 & 78.0306 & 145.1940 & 2471.20 & 0 & 25 & 0.0 \\
\hline 307650 & 78.0305 & 145.1875 & 2470.98 & 0 & 25 & 0.0 \\
\hline 307800 & 78.0304 & 145.1810 & 2470.95 & 0 & 25 & 63331.3 \\
\hline 307950 & 78.0303 & 145.1746 & 2471.07 & 0 & 25 & 0.0 \\
\hline 308100 & 78.0303 & 145.1681 & 2471.48 & 0 & 25 & 63328.3 \\
\hline 308250 & 78.0302 & 145.1616 & 2471.41 & 0 & 25 & 0.0 \\
\hline 308400 & 78.0301 & 145.1551 & 2471.41 & 0 & 25 & 0.0 \\
\hline 308550 & 78.0300 & 145.1487 & 2471.38 & 0 & 25 & 0.0 \\
\hline 308700 & 78.0300 & 145.1422 & 2471.33 & 0 & 25 & 0.0 \\
\hline 308850 & 78.0299 & 145.1357 & 2471.44 & 0 & 25 & 63328.1 \\
\hline 309000 & 78.0298 & 145.1293 & 2471.24 & 0 & 25 & 0.0 \\
\hline 309150 & 78.0297 & 145.1228 & 2471.33 & 0 & 25 & 0.0 \\
\hline 309300 & 78.0297 & 145.1164 & 2471.24 & 0 & 25 & 0.0 \\
\hline 309450 & 78.0296 & 145.1099 & 2471.41 & 0 & 25 & 0.0 \\
\hline 309600 & 78.0295 & 145.1034 & 2471.01 & 0 & 25 & 63305.1 \\
\hline 309750 & 78.0294 & 145.0970 & 2471.05 & 0 & 25 & 0.0 \\
\hline 309900 & 78.0293 & 145.0905 & 2470.92 & 0 & 25 & 0.0 \\
\hline 310050 & 78.0293 & 145.0840 & 2470.85 & 0 & 25 & 0.0 \\
\hline 310200 & 78.0292 & 145.0775 & 2470.96 & 0 & 25 & 63307.2 \\
\hline 310350 & 78.0291 & 145.0711 & 2470.93 & 0 & 25 & 0.0 \\
\hline 310500 & 78.0290 & 145.0646 & 2470.53 & 0 & 25 & 0.0 \\
\hline 310650 & 78.0290 & 145.0582 & 2470.80 & 0 & 25 & 0.0 \\
\hline 310800 & 78.0289 & 145.0517 & 2470.82 & 0 & 25 & 0.0 \\
\hline 310950 & 78.0288 & 145.0452 & 2470.47 & 0 & 25 & 63288.1 \\
\hline 311100 & 78.0287 & 145.0388 & 2470.33 & 0 & 25 & 0.0 \\
\hline 311250 & 78.0286 & 145.0323 & 2470.51 & 0 & 25 & 0.0 \\
\hline 311400 & 78.0286 & 145.0258 & 2470.36 & 0 & 25 & 0.0 \\
\hline 311550 & 78.0285 & 145.0194 & 2470.11 & 0 & 25 & 0.0 \\
\hline 311700 & 78.0284 & 145.0129 & 2469.96 & 0 & 25 & 63270 \\
\hline 311850 & 78.0283 & 145.0065 & 2469.93 & 0 & 25 & 0.0 \\
\hline 312000 & 78.0282 & 145.0000 & 2470.08 & 0 & 25 & 0.0 \\
\hline 312150 & 78.0282 & 144.9935 & 2469.95 & 0 & 25 & 0.0 \\
\hline 312300 & 78.0281 & 144.9871 & 2469.80 & 0 & 25 & 0.0 \\
\hline 312450 & 78.0280 & 144.9806 & 2470.04 & 0 & 25 & 0.0 \\
\hline 312600 & 78.0279 & 144.9741 & 2470.04 & 0 & 25 & 63250.5 \\
\hline 314700 & 78.0264 & 144.8838 & 2470.07 & 0 & & \\
\hline 316800 & 78.0248 & 144.7935 & 2472.98 & 0 & & \\
\hline 318900 & 78.0232 & 144.7033 & 2474.53 & 0 & & \\
\hline 321000 & 78.0216 & 144.6130 & 2472.95 & 0 & & \\
\hline 323100 & 78.0200 & 144.5228 & 2474.31 & 0 & & \\
\hline 325200 & 78.0183 & 144.4326 & 2472.61 & 0 & & \\
\hline 327300 & 78.0165 & 144.3425 & 2472.58 & 0 & & \\
\hline 329400 & 78.0148 & 144.2523 & 2471.97 & 0 & & \\
\hline 331500 & 78.0131 & 144.1622 & 2472.16 & 0 & & \\
\hline 333600 & 78.0113 & 144.0721 & 2474.39 & 0 & & \\
\hline
\end{tabular}




\begin{tabular}{|c|c|c|c|c|c|c|}
\hline Distance (m) & $\begin{array}{c}\text { Flag Loc. } \\
\left.\text { Latitude ( }{ }^{\circ} \mathbf{S}\right)\end{array}$ & $\begin{array}{c}\text { Flag Loc. } \\
\text { Longitude }\left({ }^{\circ} \mathrm{E}\right)\end{array}$ & Elevation (m) & $\begin{array}{c}\text { Shot } \\
\text { Number }\end{array}$ & $\begin{array}{c}\text { Julian } \\
\text { Day }\end{array}$ & $\begin{array}{l}\text { Total Mag. } \\
\text { Field (nT) }\end{array}$ \\
\hline $\begin{array}{l}335700 \\
337800 \\
339900 \\
342000 \\
344100 \\
346200 \\
348300 \\
350400 \\
352500 \\
354600 \\
356700 \\
358800 \\
360900 \\
363000 \\
365100 \\
367200 \\
369300 \\
371100 \\
371400 \\
373500 \\
375600\end{array}$ & $\begin{array}{l}78.0095 \\
78.0076 \\
78.0058 \\
78.0039 \\
78.0020 \\
78.0000 \\
77.9980 \\
77.9960 \\
77.9939 \\
77.9919 \\
77.9898 \\
77.9876 \\
77.9855 \\
77.9833 \\
77.9811 \\
77.9789 \\
77.9766 \\
77.9746 \\
77.9743 \\
77.9720 \\
77.9696\end{array}$ & $\begin{array}{l}143.9821 \\
143.8920 \\
143.8020 \\
143.7120 \\
143.6221 \\
143.5321 \\
143.4422 \\
143.3524 \\
143.2625 \\
143.1727 \\
143.0830 \\
142.9932 \\
142.9035 \\
142.8138 \\
142.7242 \\
142.6345 \\
142.5450 \\
142.4682 \\
142.4554 \\
142.3659 \\
142.2764\end{array}$ & $\begin{array}{r}2476.20 \\
2475.43 \\
2476.28 \\
2481.45 \\
2482.93 \\
2479.94 \\
2478.85 \\
2478.27 \\
2477.97 \\
2476.47 \\
2478.7 \\
2483.27 \\
2485.96 \\
2486.47 \\
2490.71 \\
2494.15 \\
2502.27 \\
2508.08 \\
2508.4 \\
2507.91 \\
2510.66\end{array}$ & $\begin{array}{l}0 \\
0 \\
0 \\
0 \\
0 \\
0 \\
0 \\
0 \\
0 \\
0 \\
0 \\
0 \\
0 \\
0 \\
0 \\
0 \\
0 \\
0 \\
0 \\
0 \\
0\end{array}$ & & \\
\hline
\end{tabular}


APPENDIX 2: Gravity Measurements

by Ron Hackney and Uri ten Brink

\begin{tabular}{|c|c|c|c|c|c|c|c|c|c|}
\hline $\begin{array}{c}J \\
\text { Day }\end{array}$ & $\begin{array}{l}\text { Time } \\
\text { (Local) }\end{array}$ & $\begin{array}{c}\text { istance } \\
(\mathrm{m})\end{array}$ & $\begin{array}{c}\text { Raw } \\
\text { Gravity }\end{array}$ & $\begin{array}{c}\text { Observed G. } \\
\text { (mGal) }\end{array}$ & $\begin{array}{c}J \\
\text { Day }\end{array}$ & $\begin{array}{c}\text { Time } \\
\text { (Local) }\end{array}$ & $\begin{array}{l}\text { Distance } \\
\text { (m) }\end{array}$ & $\begin{array}{l}\text { Raw } \\
\text { Gravity }\end{array}$ & $\begin{array}{l}\text { Observed G. } \\
\text { (mGal) }\end{array}$ \\
\hline \multirow[t]{2}{*}{344} & 53 & oha & 75.05 & & & $16: 10$ & 65850 & 5977.95 & 982290.25 \\
\hline & $18: 50$ & alpha & 5974.98 & & & $17: 45$ & 67950 & 5985.77 & 982298.06 \\
\hline \multirow[t]{2}{*}{345} & $10: 05$ & o) & 5978.38 & & 356 & 13:19 & 69900 & 5977.22 & 982289.44 \\
\hline & 19:15 & 0 & 5978.44 & & & $14: 32$ & 71550 & 5972.80 & 982285.00 \\
\hline \multirow[t]{6}{*}{346} & $10: 12$ & 0 & 5978.38 & 982292.00 & & $16: 05$ & 73650 & 5978.32 & 982290.50 \\
\hline & $12: 50$ & 1050 & 5981.18 & & 361 & $14: 14$ & 75750 & 5983.49 & 982295.06 \\
\hline & $14: 30$ & 2100 & 5982.57 & 982296.06 & & 15:05 & 77850 & 5976.68 & 982288.25 \\
\hline & $16: 15$ & 3450 & 5987.97 & 982301.44 & & $15: 41$ & 79950 & 5972.42 & 982284.00 \\
\hline & $18: 45$ & 5550 & 5983.15 & 982296.63 & & $16: 08$ & 81150 & 5965.36 & 982276.94 \\
\hline & $21: 15$ & 6750 & 5979.12 & & & $16: 37$ & 82500 & 5957.74 & 982269.31 \\
\hline \multirow[t]{5}{*}{347} & $12: 30$ & 7500 & 5978.37 & 982291.75 & & $17: 12$ & 84600 & 5947.82 & 982259.38 \\
\hline & $14: 00$ & 9600 & 5976.43 & 982289.81 & & $17: 47$ & 86700 & 5939.67 & 982251.19 \\
\hline & $15: 20$ & 10500 & 5974.67 & 982288.00 & & 18:25 & 88800 & 5936.65 & 982248.19 \\
\hline & $17: 00$ & 12600 & 5961.82 & 982275.19 & & 19:57 & 90500 & 5936.56 & \\
\hline & $18: 45$ & 14700 & 5955.57 & 982268.88 & 362 & 10:05 & 90900 & 5936.60 & 982248.06 \\
\hline \multirow[t]{5}{*}{348} & $11: 00$ & 15900 & 5955.23 & 982268.50 & & 11:02 & 93000 & 5936.55 & 982248.00 \\
\hline & 11: & 18000 & 5959.35 & 982272.56 & & $12: 00$ & 94800 & 5933.24 & 982244.69 \\
\hline & $13: 30$ & 20100 & 5962.96 & 982276.19 & & $13: 15$ & 96900 & 5930.76 & 982242.19 \\
\hline & $15: 35$ & 22200 & 5971.88 & 982285.13 & & $14: 54$ & 99000 & 5926.89 & 982238.31 \\
\hline & $16: 25$ & 23100 & 5973.15 & & & $16: 08$ & 101100 & 5919.99 & 982231.44 \\
\hline \multirow[t]{2}{*}{349} & $12: 20$ & 24000 & 5975.37 & 982288.50 & & $17: 15$ & 103200 & 5916.71 & 982228.13 \\
\hline & $13: 50$ & 26100 & 5984.05 & 982297.13 & & $18: 25$ & 105300 & 5915.96 & 982227.38 \\
\hline \multirow[t]{6}{*}{351} & $12: 04$ & 28200 & 5985.97 & 982298.81 & 1 & $10: 40$ & 105200 & 5915.86 & \\
\hline & $13: 05$ & 29700 & 5985.23 & 982298.06 & & $12: 24$ & 107400 & 5916.04 & 982227.13 \\
\hline & $14: 30$ & 31800 & 5982.72 & 9822 & & $13: 30$ & 109500 & 5916.23 & 982227.25 \\
\hline & $16: 13$ & 33900 & 5980.49 & 982293.31 & & $14: 42$ & 111600 & 5917.28 & 982228.31 \\
\hline & $16: 55$ & 34800 & 5979.06 & 9822 & & $16: 35$ & 113700 & 5917.27 & 982228.31 \\
\hline & $18: 37$ & 36900 & 5975.43 & 982288.25 & & $17: 50$ & 115800 & 5916.44 & 982227.44 \\
\hline \multirow[t]{7}{*}{353} & $15: 50$ & 38000 & 5968.45 & 982275.94 & & 19:08 & 117900 & 5917.64 & 982228.69 \\
\hline & $17: 17$ & 41100 & 5963.34 & 982274.81 & & $20: 35$ & 120000 & 5917.32 & 982228.31 \\
\hline & $18: 05$ & 42150 & 5962.27 & & 2 & $14: 23$ & 122100 & 5916.88 & 982227.81 \\
\hline & $19: 06$ & 43350 & 5962.27 & 982274.81 & & 15:35 & 124200 & 5919.94 & 982230.88 \\
\hline & $19: 55$ & 44550 & 5962.59 & 982275.13 & & $17: 07$ & 126300 & 5922.05 & 982228.50 \\
\hline & $20: 43$ & 45600 & 5962.16 & 982274.69 & & $18: 10$ & 127950 & 5917.62 & 982223.81 \\
\hline & $21: 20$ & 46650 & 5961.35 & 982273.88 & & $20: 40$ & bravo & 5915.48 & \\
\hline \multirow[t]{6}{*}{354} & $13: 54$ & 48750 & 5956.52 & 982269.00 & 3 & $12: 12$ & 129750 & 5912.98 & \\
\hline & $15: 21$ & 50700 & 5953.84 & 982266.31 & & $13: 20$ & 131850 & 5906.73 & 982217.50 \\
\hline & $16: 35$ & 52800 & 5954.68 & 982267.13 & & $14: 35$ & 133950 & 5902.54 & 982212.75 \\
\hline & $17: 35$ & 54600 & 5958.39 & 982270.81 & & $15: 40$ & 136050 & 5900.84 & 982211.00 \\
\hline & $19: 05$ & 56700 & 5962.95 & 982275.38 & & $17: 25$ & 138150 & 5901.12 & 982211.25 \\
\hline & $20: 22$ & 58800 & 5965.88 & 982278.31 & & $18: 35$ & 140250 & 5902.13 & 982212.31 \\
\hline \multirow[t]{3}{*}{355} & $13: 15$ & 60900 & 5967.24 & 982279.56 & & $19: 43$ & 142350 & 5903.35 & 982213.50 \\
\hline & $14: 20$ & 63150 & 5969.45 & & & $20: 38$ & 143850 & 5903.80 & 982213.94 \\
\hline & 15:05 & 64350 & 5972.81 & 982285.13 & $\boldsymbol{I}$ & 13:55 & 145950 & 5904.04 & 982214.06 \\
\hline
\end{tabular}




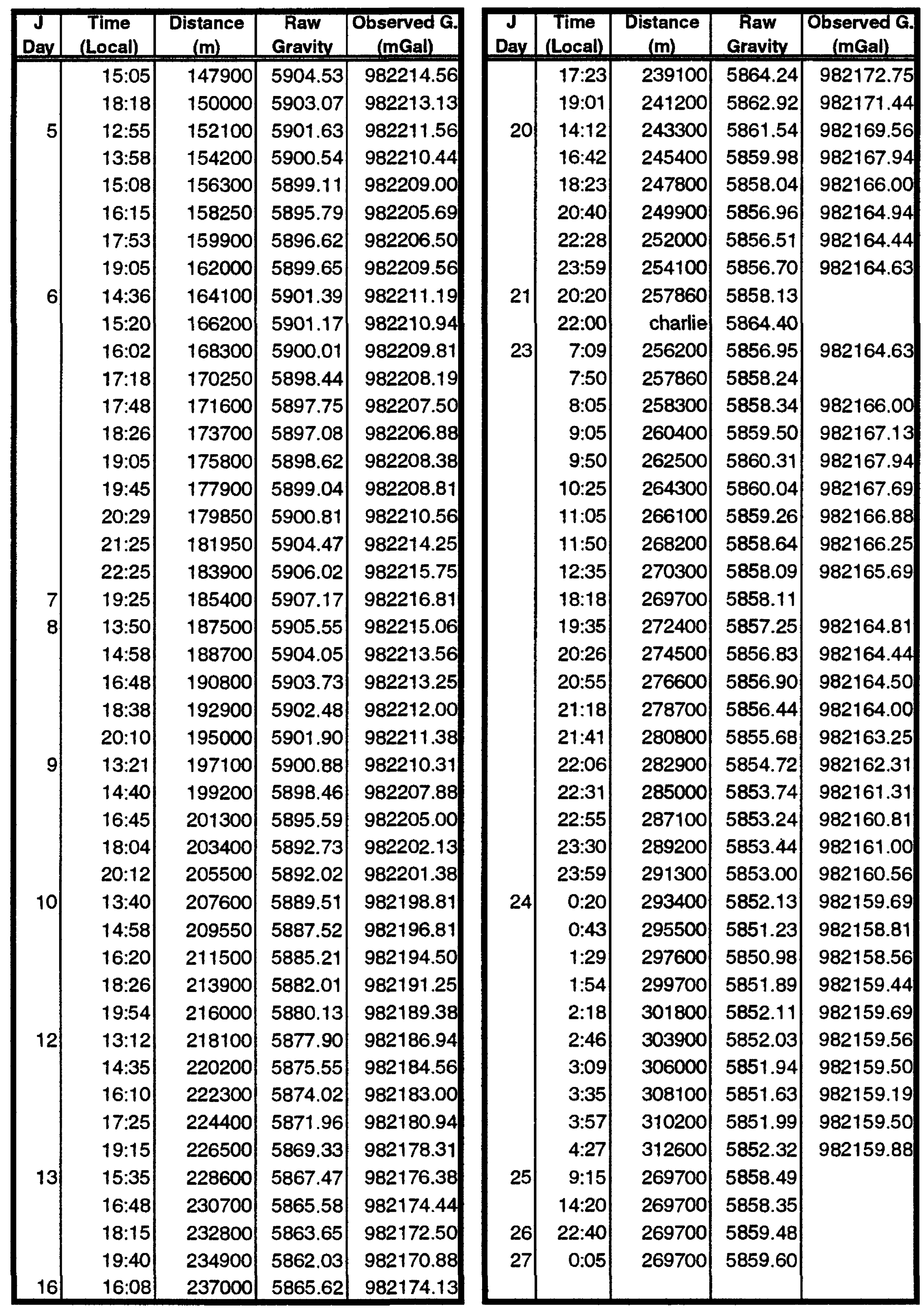




\title{
APPENDIX 3: Ground Penetrating Radar Measurements
}

\author{
By Yizhaq Makovsky
}

\section{INTRODUCTION}

The main purpose of the radar component of this project was to image the ice-rock interface to provide independent information for seismic processing. This was particularly important at the beginning of the traverse, near the mountains, where significant topography of the ice-rock interface was expected. Good penetration, at the expense of resolution, was required to reach the ice-rock interface at depths exceeding $1 \mathrm{~km}$. To achieve the penetration needed we had to use a low frequency $(\sim 1 \mathrm{MHz})$ radar system. However, such system has long $(40 \mathrm{~m})$ antennae that make it cumbersome to use. The radar data had to be collected with little effort and demand as little time as possible from the team, because seismic profiling was the main effort of the experiment.

An automated profiling.radar system, designed after a system built by Ed Waddington and Dave Morse of the University of Washington, allowed us to acquire low frequency radar data without affecting the progress of the seismic experiment. The system was put together at the last moment with components of different origins and with the help of Ed Waddington and Dave Morse. This system is described by Weertman (1993) and is principally based on the procedure described by Watts and Wright (1981) modified to be operated by a single person.

\section{SYSTEM CONFIGURATION AND FIELD PROCEDURES}

\section{General set-up}

The radar system included a transmitter unit and a receiver unit, each connected to a twoarm wire antenna and each located on a separate sled (Figure A3.1). The two antennae were positioned in an endfire parallel alignment separated by a distance on the order of an antenna half length. The antennae, sleds, and the towing Alpine-II Skidoo snowmobile were chained to each other with stiff non-conducting ropes that kept the system spread for acquisition while it was towed. The receiver needed to be checked more frequently, and was therefore, towed in front (closer to the snowmobile driver).

\section{Antennae}

Principles: Both transmitting and receiving antennae are identical center-fed dipole wireantennae symmetric about the feed point and are resistivity-loaded. The resistive load attenuates the outgoing waves and prevents a reflection of the wave inward from the tips of the antenna. The resistance per unit length at a distance $z$ (in meters) inward from the outer end of the antenna arm is

$\mathrm{R}(\mathrm{z})=¥ / \mathrm{z}$

where $¥$ is the resistive loading constant in ohms.

The center frequency of the transmitted pulse, for antennae lying on the ice surface, is approximately given by

$\mathrm{f}=50 / \mathrm{h}$

where $h$ is the antenna half length in meters and $\mathrm{f}$ is the center frequency in $\mathrm{MHz}$.

Specifications: We used antennae with half length $h=40 \mathrm{~m}$, center frequency $f=1.25 \mathrm{~Hz}$ and resistivity $¥=400 \mathrm{ohm}$ lent to us by Ed Waddington and Dave Morse. 


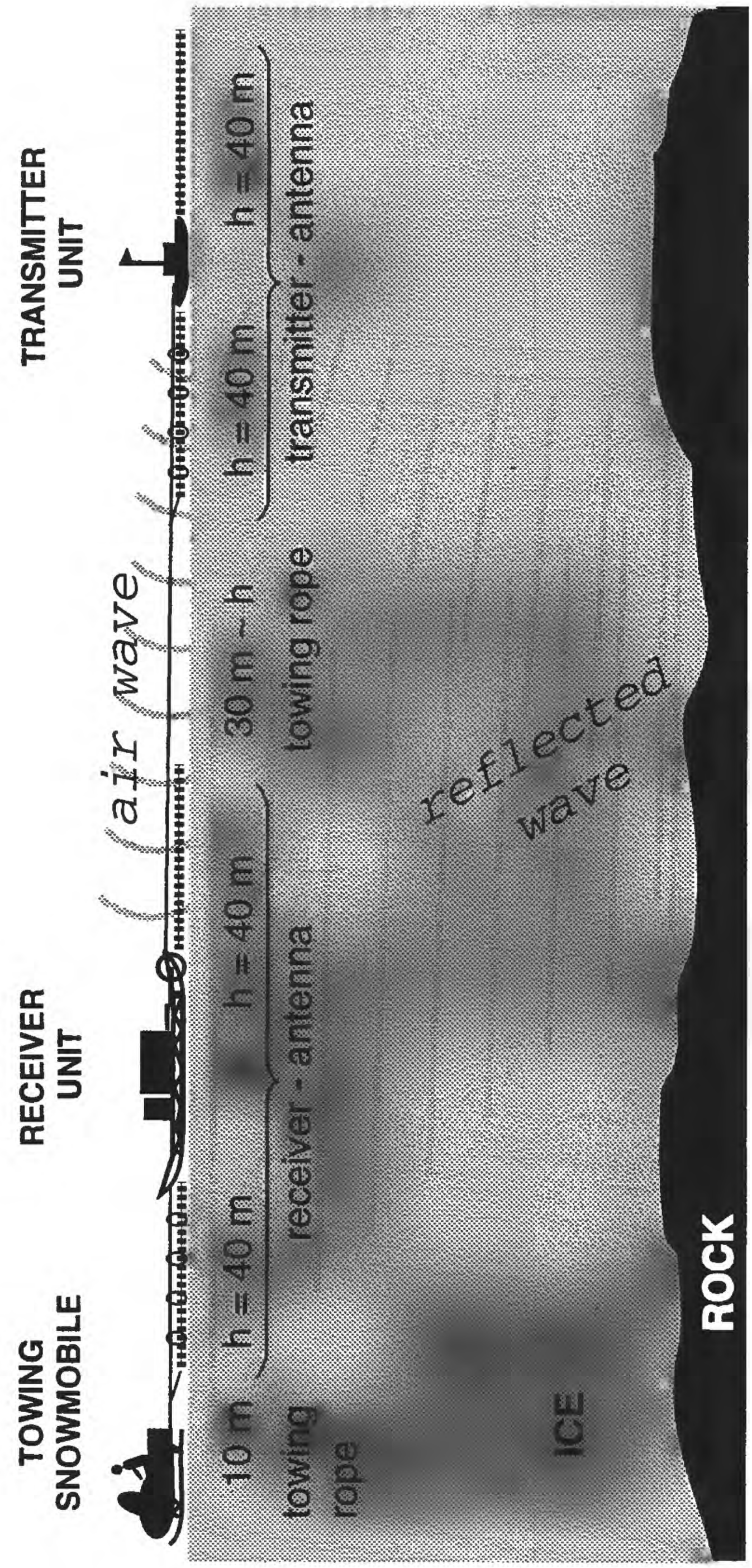

क्ष

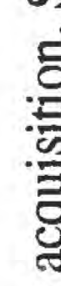

垔

온

营

兘

음

4

()

\&

웅

동

恶

S

ले

氙 
Field set-up: The backward-directed arms of the antennae were thin conducting wires, which dragged behind the sleds. The forward-directed arms of the antennae were inserted into 1 " webbing to avoid tension on the antennae. The webbing was tied at its two ends with $3 / 8$ " bungie cord to the towing rope, and the bungie cord acted as a shock absorber. The webbing was passed through carabbiners, tied at constant intervals to the towing rope, to keep the webbing parallel with and close to the towing rope.

\section{Transmitter unit}

Components: The transmitter unit, described in Figure A3.2a, had two components: a high voltage converter and a pulse generator. The converter provided an adjustable, $750 \mathrm{~V}$ power supply from a $12 \mathrm{~V}$ battery to the pulse generator. The pulse repetition rate of the generator was controlled by the power supply current and was normally set at a few $\mathrm{KHz}$. The pulse generator used was a four-stage transistor-avalanche as described by Watts and Wright (1981) with about $600 \mathrm{~V}$ output. The converter and pulse generator were lent to us by Steve Hodge, Water Resource Division, USGS.

Field set-up: The pulse generator and converter were soft packed together with one 50 Ah $12 \mathrm{~V}$ Sealed-Lead-Acid battery into a wooden box. Thanks to the large battery capacity, this unit seldom needed to be charged. The transmitter was mounted on a small "Banana" sled. Because the "Banana" sled could easily flip over, a flag-pole was placed on top of the sled to allow the operator $(160 \mathrm{~m}$ in front of the sled) to see whether it was upright while driving (Figure A3.1).

\section{Receiver unit}

The receiver unit, illustrated in Figure $\mathrm{A} 3.2 \mathrm{~b}$, was a combination of two computercontrolled assemblages, the triggering assemblage and the acquisition assemblage. The triggering assemblage measured the progression over the ground and passed the information in a digitized form to the computer that acquired a trace when a pre-specified distance elapsed. In the acquisition assemblage, the signal received from the antenna was amplified, digitized, stacked, and passed to the computer.

\section{The triggering assemblage:}

Outline: A bicycle wheel was dragged behind the receiver sled. A magnetic dip-switch, connected to an electronic counter box, counted the wheel rotations and the count was transferred to the parallel port of the computer. The computer reset the counter every time a trace was acquired.

\section{The acquisition assemblage:}

Outline: The signal from the antenna arms was received by an amplifier, then passed to both input channels of a digital oscilloscope. As in most ground penetrating radar, the oscilloscope was triggered by the first arriving wave, the air wave, which is also the strongest arrival (a small pre-trigger length was used to verify the recording of the first arrival). The traces were averaged by the oscilloscope and the resulting trace was transmitted to the computer, together with the scope settings (through the communication port), upon request from the computer.

Specifications and parameters: We used an amplifier with a scalar gain of about 13, lent to us by Ed Waddington and Dave Morse. We began the survey using a Tektronix 222 Power Scout $10 \mathrm{MHz}$ digital storage scope that was later changed to a Fluke 97 Scopemeter. Both oscilloscopes output a digital trace with 512 one-byte samples. This sampling produces data which are strictly limited both in terms of dynamic range and frequency response. 
a. Transmitter unit

wooden box

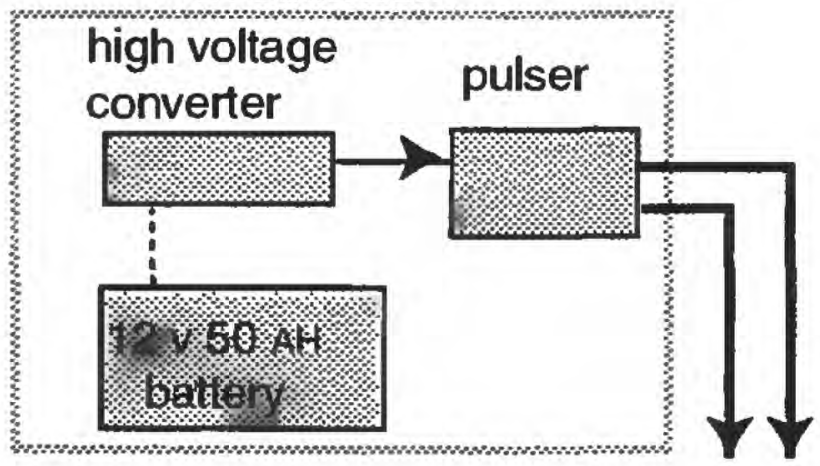

transmitter antenna

b. Receiver unit

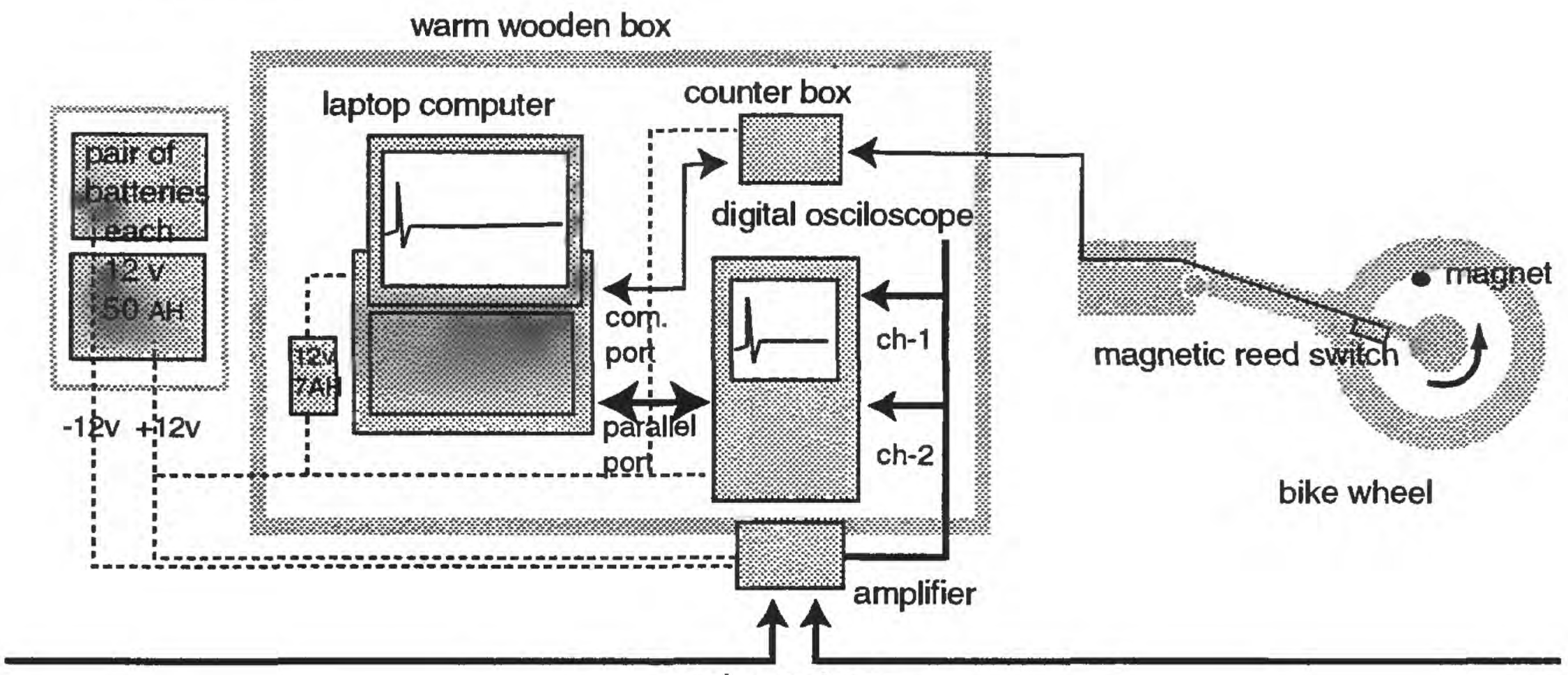

receiver antenna

Figure A3.2 Diagrams describing the components of the ground penetrating radar system. Arrows indicate the directions of signal transmission, dashed lines describe DC power supply, thick lines connect the acquisition receiver assemblage components, and thin lines connect the receiver triggering assemblage components. 
We used $2 \mu \mathrm{sec} /$ division time scale on the scope which translates to $0.08 \mu$ sec sample interval, $12.5 \mathrm{MHz}$ sample rate, about $3 \mathrm{MHz}$ Nyquist frequency, and trace length of 40.88 $\mu \mathrm{sec}$ (equivalent to a depth of about $3400 \mathrm{~m}$ in ice). To get around the limited dynamic range, channel 2 of the oscilloscope was set to a low amplitude gain, sampling the first arrival properly, and was used to trigger the oscilloscope. Channel 1 was set to a much higher gain (2-20 mV/division) to sample the small secondary arrivals (reflected waves) properly, while clipping the first arrivals. This channel was used as the data-acquisition channel. The oscilloscope was set-up in the 'average' mode to stack subsequent traces. The Tek 222 was able to stack 128 subsequent traces, and the Fluke 97 was able to stack 256 traces.

\section{The computer:}

Outline: The receiver unit was controlled by a program running on a PC laptop. The program was launched at the beginning of acquisition and acquired data while the operator was driving the towing snowmobile. A trace was acquired from the scope when the number of wheel revolutions, received by the program, equaled the 'counts per trace' value in the program configuration file. The count was then reset. The traces were kept on RAM during acquisition, and were own-loaded at the end of an acquisition session. The data were recorded with headers describing the scope configuration, location, and wheel count for each trace.

Details: The two oscilloscopes used different programs. The program used with the Fluke 97 oscilloscope required a math-coprocessor (FPU) whereas the program used by the Tek 222 did not. The FPU was not available on the 286 Toshiba laptop initially used, but starting at Julian day 353 we were able to run the Fluke 97 program on an Avanti 486 laptop with an FPU. The program used with the Fluke 97 was more advanced. It allowed the operator to enter the location along the line at the start and the end of acquisition and when he stopped to check the radar operation.

\section{Field set-up:}

Weather protection: Both the oscilloscope and laptop had LCD screens which needed to be shielded from freezing. They were put in an insulated wooden box with hot water bottles containing about 10 liter in total. (The bottles were insulated to slow the heat flux). The wooden box had an external lid and an internal transparent lid (Perspex sheet). The transparent lid had openings to handle the receiver without exposing the contents of the box to the cold.

Power supply: The amplifier needed $\pm 12 \mathrm{~V}$ power supply whereas the rest of the components operated with a $12 \mathrm{~V}$ supply. Power was supplied to the receiver unit from two $12 \mathrm{~V} 50$ Ah sealed lead-acid batteries that were packed into a separate wooden box and charged once every few days. The Avanti laptop needed $14 \mathrm{~V}$ supply, so another $12 \mathrm{~V} 7$ Ah battery with a DC converter was added into the oscilloscope and laptop box when this laptop was used. Although this battery was charged daily, its capacity put a limit on the duration of acquisition, and several times even caused data loss .

Packing: The wooden components and batteries boxes were strapped on top of a "Nansen" sled. The bike-wheel fork was mounted in the back of the sled allowing the wheel some freedom to move up and down to follow the terrain. The antenna arms were kept out of the way of the sled.

\section{Field procedure}

The radar was operated only when it was far from other traverse vehicles to prevent reflections off the other vehicles and especially, the seismic streamer. The radar equipment stayed at the base camp, and followed at a distance $>200 \mathrm{~m}$ behind the base camp, when 
the camp moved at the end of the working day. Hot water bottles were prepared right before the camp was moved, the receiver was arranged, the source started, the oscilloscope configuration was set-up to receive a stable signal, the acquisition program was started, the location inserted, and finally, the snowmobile was connected to the towing rope, and the radar tow began.

The radar was towed at a speed of up to $20 \mathrm{~km} / \mathrm{h}$, while acquisition took place. Once in a while, the operator stopped the radar and checked the receiver to verify that the acquisition was stable and to input the receiver-sled surface location. When a large distance was traversed in a day, the program was stopped and the data file was saved to disk. Acquisition was then restarted, recording into another file.

As the radar approached camp, it was aligned with the streamer (producing resonating reflections off the streamer that could be clearly seen on the last traces in most acquisition sessions). After the radar was parked, the program was stopped and the data were saved to disk, and briefly viewed for quality control, then backed-up to floppy disk. The receiver components were turned off and taken to a warm place, and the source was turned off.

Part of the section between Taylor Dome $(\mathrm{km} \mathrm{-10)}$ and $\mathrm{km} 68.450$ was acquired with the Tek 222-based system. The entire section was acquired again with the Fluke 97 basedsystem during a re-supply trip to Taylor Dome. During this trip, the section between $\mathrm{km}$ 68.450 and $\mathrm{km} 18$ was recorded on the way to Taylor Dome and the section between Taylor Dome and km 18 was recorded on the way back. During this trip, the radar system was towed behind the Tucker Snocat 069 at a speed of 3-4 km.

\section{DATA REDUCTION}

A total of 18 data files, covering (with some overlap and some gaps) a total section of 205 $\mathrm{km}$, were recorded in 14 days of acquisition. Of these files, 4 were recorded with the Tek 222 based system and 12 were recorded with a Fluke 97 based system. The Fluke 97 data covers all the section recorded with Tek 222 system. To date, only the Fluke 97 files have been reduced.

Data were converted into SEG-Y format to allow the use of standard seismic processing software, and to facilitate the viewing and processing of the data. First the data were stripped of their headers with the University of Washington software (lent to us by Dave Morse). The headers were interpreted and the wheel counts were converted into distance and compared with the surface locations input by the operator in the field. We found that the wheel counts underestimated the distance by about $15 \%$ probably because of wheel decoupling from the surface (due to slippage or where the sled crossed a sastrugi and lifted the wheel off the surface). The trace surface-locations were linearly interpolated for each trace from the surface-locations inserted in the field.

The trace binary data were converted into SEG-Y 2 byte/sample traces. SEG-Y reel and trace headers were concatenated to the data. SEG-Y trace-headers were loaded as follows (all values are integers):

Header value surface-location in tens of meter Julian day wheel counts per trace pre-trigger length in $1 / 100 \mu \mathrm{sec}$ $\mathrm{mV} /$ division bytes in header

21-24

$159-160$

161-162

163-164

121-122 
To make the data appear to seismic processing packages as standard seismic data input, the time scale was multiplied by a factor of $1 \times 10^{5}$ (i.e., apparent sample interval of $8 \mathrm{msec}$ ). The change of time scale must be taken into consideration at all stages of processing. ProMAX ${ }^{\mathrm{TM}}$ seismic processing package, from Advance Geophysical Corporation was used to view and process the data.

\section{SUMMARY}

Using an automated ground penetrating radar system we were able to profile the ice sheet from the Transantarctic mountains to the edge of Wilkes subglacial basin. The data were acquired by a single operator placing little demand on the limited resources of the EAST93 seismic project. The resulting image reveals rough topography at the base of the ice sheet and flow patterns within the ice that wrap around basement highs.

\section{REFERENCES}

Morse, D. L. and E. D. Waddington, 1993, Glacier geophysical studies at Taylor Dome: year three., Antarctic Journal of the U.S., 28, p. 67-69.

Narod, B. B. and G. K. C. Clarke, 1994, Instruments and Methods: Miniature high-power impulse transmitter for radio-echo sounding., Journal of Glaciology, 40, p. 190-194.

Watts, R. D. and D. L. Wright, 1981, Systems for measuring thickness of temperate and polar ice from the ground or from the air., J. Glaciol., 27, p. 459-469.

Weertman, B., 1993, Interpretation of ice sheet stratigraphy: a radio echo sounding study of Dyer Plateau, PhD dissertation thesis, Graduate Program in Geophysics, University of Washington..

Wright, D. L., S. M. Hodge and R. D. Watts, 1990, Airborne radar sounding in temperate and polar ice, U.S. Geological Survey Bulletin, 1925, Developments and Applications of Modern Airborne Electromagnetic Surveys, p. 171-180. 


\title{
APPENDIX 4: Ground Conditions
}

\author{
By Ron Hackney
}

NOTE: description of the terrain encountered was not begun until December 27 th (Julian Day 361)

$74.85-90.45 \mathrm{~km}$ :

surface $=40 \%$ sastrugi $(<20 \mathrm{~cm}), 40 \%$ flat snow, $20 \%$ drift snow Note that tracks left by survey team skidoos all exist on the flat areas of snow, suggesting that many of the drifts seen formed within a period of roughly a week.

$90.9-93.0 \mathrm{~km}:$

surface $=$ mostly relatively flat and soft snow, $20 \%$ sastrugi $(<10 \mathrm{~cm})$

93.0- $103.2 \mathrm{~km}$ :

surface $=30 \%$ sastrugi $(<10 \mathrm{~cm})$, some harder and rougher snow in places some sastrugi reaching $20 \mathrm{~cm}$

$103.2-107.4 \mathrm{~km}$ :

poor definition meant difficult to examine, but mostly soft snow that is soft to drive over

$107.4-113.7 \mathrm{~km}:$

surface $=20 \%$ sastrugi $(<20 \mathrm{~cm})$, mostly soft rolling snow drifts

$113.7-117.9 \mathrm{~km}:$

surface becoming rougher and bumpier, some drift ridges up to $50 \mathrm{~cm}$ high otherwise no change

$117.9-120.6 \mathrm{~km}$ :

becoming smoother again. sastrugi $=20 \%(<20 \mathrm{~cm})$

$122.1-123.6 \mathrm{~km}:$

surface $=30 \%$ sastrugi (up to $40 \mathrm{~cm}$ high), $10 \%$ hard ice, mostly surface is firm and bumpy to drive over.

$123.6-126.3 \mathrm{~km}$ :

very large sastrugi appear, some up to $1.5 \mathrm{~m}$ from top to bottom! (the biggest encountered on the traverse); fortunately largest sastrugi occur infrequently, i.e.. they were generally easy to avoid.

$126.3-127.75 \mathrm{~km}:$

extreme sastrugi disappear, but still exist up to $50 \mathrm{~cm}$ in height; surface still rough with frequent solid ice patches.

$127.5 \mathrm{~km}$ :

surface $=10-20 \%$ sastrugi $(<30 \mathrm{~cm}), 50 \%$ solid ice patches.

$129.95-136.05 \mathrm{~km}$ :

surface $=50 \%$ hard, icy snow, $20 \%$ sastrugi $(<30 \mathrm{~cm})$, occasional snow drift up to $50 \mathrm{~cm}$. 
136.05- $143.85 \mathrm{~km}$ :

less hard snow, now only around $20 \%$; icy ridges up to $50 \mathrm{~cm}$ becoming slightly more abundant

$143.85-150.0 \mathrm{~km}:$

surface becoming bumpier due to frequent mounds of icy snow and occasional sastrugi up to $1 \mathrm{~m}$ in height.

$150.0-152.1 \mathrm{~km}:$

big sastrugi becoming less frequent, ground still firm and rough to drive over.

$152.1-162.0 \mathrm{~km}$ :

surface $=70 \%$ soft snow, $10 \%$ hard snow, $20 \%$ sastrugi $(<30 \mathrm{~cm})$; surface much smoother to drive over.

$162.0-185.4 \mathrm{~km}:$

surface $=20-40 \%$ sastrugi $(<50 \mathrm{~cm})$. Some quite rough stretches lasting $2-3 \mathrm{~km}$; rare sastrugi up to $1 \mathrm{~m}$ high; abundant steel hard icy patches.

$185.4-187.5 \mathrm{~km}$ :

extremely rough surface; sastrugi up to $1 \mathrm{~m}$, dangerous to drive over in conditions with poor definition

$187.5-190.8 \mathrm{~km}$ :

surface $=30-50 \%$ sastrugi (up to $1 \mathrm{~m}$, mostly $50 \mathrm{~cm}$ ); snow mostly soft

$190.8-194.1 \mathrm{~km}:$

large sastrugi less common

$194.1-199.2 \mathrm{~km}:$

Surface $=50 \%$ sastrugi $(<50 \mathrm{~cm})$ making driving rougher

$199.2-205.5 \mathrm{~km}:$

surface $=20-30 \%$ sastrugi $(<50 \mathrm{~cm}$, rarely to $1 \mathrm{~m})$; surface smoother to travel over.

$205.5-224.4 \mathrm{~km}:$

surface $=20-40 \%$ sastrugi $(<50 \mathrm{~cm}), 10 \%$ hard snow, smooth to travel over.

$224.4-235.5 \mathrm{~km}$ :

surface $=10-20 \%$ sastrugi $(<30 \mathrm{~cm})$.

235.5 - $241.5 \mathrm{~km}:$

occasional snow drift reaching $1 \mathrm{~m}$ in height

$241.5-254.7 \mathrm{~km}:$

surface $=20-50 \%$ sastrugi (mostly $20-50 \mathrm{~cm}$ ), remainder is snow drifts and ridges.

$254.7-280.0 \mathrm{~km}$ :

no observations recorded, mostly was quite flat

$280.0-312.5 \mathrm{~km}$ :

surface $=$ dead flat, no topography, sastrugi $<10 \%(<10 \mathrm{~cm})$. 
APPENDIX 5: Weather Observations by the Main Party

by Ron Hackney and Rebecca Drury

\begin{tabular}{|c|c|c|c|c|c|c|c|c|}
\hline $\begin{array}{c}\downarrow \\
\text { Day }\end{array}$ & $\begin{array}{c}\text { Time } \\
\text { (local) }\end{array}$ & $\begin{array}{c}\text { Distance } \\
(\mathrm{m})\end{array}$ & $\begin{array}{c}\text { Temp } \\
\left({ }^{\circ} \mathrm{C}\right)\end{array}$ & $\begin{array}{c}\mathrm{P1} \\
\text { (mBar) }\end{array}$ & $\begin{array}{c}\text { P2 } \\
\text { (mBar) }\end{array}$ & $\begin{array}{l}\text { Wind } \\
\text { Speed } \\
(\mathbf{k m} / \mathbf{h})\end{array}$ & $\begin{array}{l}\text { Wind } \\
\text { Dir. }\end{array}$ & Comments \\
\hline 344 & \begin{tabular}{r|}
$0: 03$ \\
$12: 45$ \\
$14: 53$ \\
$18: 50$ \\
\end{tabular} & & $\begin{array}{r}-20 \\
-19 \\
\end{array}$ & $\begin{array}{l}721.99 \\
721.32 \\
721.30 \\
721.55 \\
\end{array}$ & $\begin{array}{l}721.10 \\
720.21 \\
720.06 \\
720.51 \\
\end{array}$ & $\begin{array}{r}12 \\
14 \\
12 \\
\end{array}$ & $\begin{array}{l}S \\
S \\
S\end{array}$ & $\begin{array}{l}\text { overcast, low clouds, occasional } \\
\text { light snow }\end{array}$ \\
\hline 345 & $\begin{array}{l}10: 05 \\
19: 15 \\
\end{array}$ & $\begin{array}{l}0 \\
0\end{array}$ & $\begin{array}{l}-24 \\
-22 \\
\end{array}$ & $\begin{array}{l}725.19 \\
723.94 \\
\end{array}$ & $\begin{array}{l}724.60 \\
723.30 \\
\end{array}$ & $\begin{array}{r}0 \\
14 \\
\end{array}$ & sw & clear and sunny \\
\hline 346 & $\begin{array}{l}10: 12 \\
12: 50 \\
14: 30 \\
15: 40 \\
16: 15 \\
18: 45 \\
21: 15 \\
\end{array}$ & $\begin{array}{r}0 \\
1050 \\
2100 \\
3000 \\
3450 \\
5550 \\
6750 \\
\end{array}$ & $\begin{array}{l}-26 \\
-23 \\
-22 \\
-21 \\
-22 \\
-21 \\
-24\end{array}$ & $\begin{array}{l}722.36 \\
721.92 \\
721.66 \\
721.81 \\
722.16 \\
723.57 \\
723.78 \\
\end{array}$ & $\begin{array}{l}721.30 \\
720.99 \\
721.13 \\
720.92 \\
721.13 \\
722.58 \\
722.97 \\
\end{array}$ & 21 & WSW & clear and sunny \\
\hline 347 & $\begin{array}{r}9: 30 \\
12: 30 \\
14: 00 \\
15: 20 \\
17: 00 \\
18: 45 \\
\end{array}$ & $\begin{array}{r}6750 \\
7500 \\
9600 \\
10500 \\
12600 \\
14700 \\
\end{array}$ & $\begin{array}{l}-25 \\
-23 \\
-23 \\
-22 \\
-21 \\
-23 \\
\end{array}$ & $\begin{array}{l}724.65 \\
725.17 \\
725.53 \\
726.07 \\
726.27 \\
726.26 \\
\end{array}$ & $\begin{array}{l}724.16 \\
724.19 \\
724.60 \\
725.34 \\
726.03 \\
725.76 \\
\end{array}$ & 0 & NNE & $\begin{array}{l}\text { scattered high clouds, mountains to } \\
\text { east clouded, occaisional } \\
\text { low, foggy clouds }\end{array}$ \\
\hline 348 & $\begin{array}{l}11: 00 \\
11: 40 \\
13: 30 \\
15: 35 \\
16: 25 \\
\end{array}$ & $\begin{array}{l}15900 \\
18000 \\
20100 \\
22200 \\
23100 \\
\end{array}$ & \begin{tabular}{l|}
-24 \\
-24 \\
-23 \\
-22 \\
-18 \\
\end{tabular} & $\begin{array}{l}726.89 \\
727.85 \\
727.99 \\
728.54 \\
728.53 \\
\end{array}$ & $\begin{array}{l}726.27 \\
727.07 \\
727.27 \\
727.86 \\
727.81 \\
\end{array}$ & $\begin{array}{l}-22 \\
-19 \\
\sim 19 \\
\sim 19 \\
-28 \\
\end{array}$ & $\begin{array}{l}W \\
W \\
W \\
W \\
W\end{array}$ & \\
\hline 349 & $\begin{array}{l}12: 20 \\
13: 50 \\
\end{array}$ & $\begin{array}{l}24000 \\
26100 \\
\end{array}$ & $\begin{array}{l}-23 \\
-21 \\
\end{array}$ & $\begin{array}{l}728.83 \\
730.12 \\
\end{array}$ & $\begin{array}{l}727.99 \\
729.07 \\
\end{array}$ & $\begin{array}{r}-19 \\
-22 \\
\end{array}$ & $\begin{array}{l}\text { W } \\
W\end{array}$ & clear and sunny \\
\hline 350 & & 24300 & & & & 32 & $W$ & clear and sunny, with blowing snow \\
\hline 351 & $\begin{array}{c}12: 04 \\
13: 05 \\
14: 30 \\
16: 13 \\
16: 55 \\
18: 37 \\
\end{array}$ & $\begin{array}{l}28200 \\
29700 \\
31800 \\
33900 \\
34800 \\
36900 \\
\end{array}$ & $\begin{array}{l}-19 \\
-18 \\
-17 \\
-15 \\
-15 \\
-15 \\
\end{array}$ & $\begin{array}{l}743.52 \\
743.91 \\
745.00 \\
745.39 \\
746.07 \\
749.43 \\
\end{array}$ & $\begin{array}{l}741.30 \\
742.85 \\
743.99 \\
744.57 \\
745.50 \\
748.86 \\
\end{array}$ & $\begin{array}{r}0 \\
\sim 8 \\
-8 \\
-5 \\
\sim 10 \\
\sim 15 \\
\end{array}$ & $\begin{array}{l}\text { SW } \\
S \\
S \\
S \\
S \\
\end{array}$ & $\begin{array}{l}\text { cover of high cloud, often snowing } \\
\text { with poor surface definition } \\
\text { and reduced visibility }\end{array}$ \\
\hline 352 & & 35700 & -17 & & & 23 & $s$ & snowing with low visibility \\
\hline 353 & $\begin{array}{l}11: 13 \\
15: 50 \\
17: 17 \\
18: 05 \\
19: 06 \\
19: 55 \\
20: 43 \\
21: 20\end{array}$ & $\begin{array}{l}35450 \\
38000 \\
41100 \\
42150 \\
43350 \\
44550 \\
45600 \\
46650\end{array}$ & $\begin{array}{l}-13 \\
-14 \\
-14 \\
-13 \\
-13 \\
-13 \\
-12 \\
-10\end{array}$ & $\begin{array}{l}745.11 \\
746.27 \\
745.12 \\
744.77 \\
743.93 \\
743.54 \\
743.22 \\
742.90\end{array}$ & $\begin{array}{l}744.55 \\
745.36 \\
744.44 \\
743.95 \\
743.27 \\
742.85 \\
742.58 \\
742.44\end{array}$ & $\begin{array}{r}-13 \\
-12 \\
\sim 12 \\
\sim 10 \\
\sim 10 \\
\sim 5 \\
\sim 5 \\
\sim 2\end{array}$ & $\begin{array}{l}\text { SSW } \\
\text { SW } \\
\text { SSW } \\
\text { S } \\
\text { S } \\
\text { SW } \\
\text { SW } \\
\text { W }\end{array}$ & $\begin{array}{l}\text { scattered high clouds and low clouds } \\
\text { drifting from south (variable } \\
\text { visibility and surface definition) }\end{array}$ \\
\hline
\end{tabular}




\begin{tabular}{|c|c|c|c|c|c|c|c|c|}
\hline $\begin{array}{c}J \\
\text { Day }\end{array}$ & $\begin{array}{c}\text { Time } \\
\text { (local) }\end{array}$ & $\begin{array}{c}\text { Distance } \\
(\mathrm{m})\end{array}$ & $\begin{array}{c}\text { Temp } \\
\left({ }^{\circ} \mathrm{C}\right)\end{array}$ & $\begin{array}{c}\text { P1 } \\
\text { (mBar) }\end{array}$ & $\begin{array}{c}\mathbf{P 2} \\
(\mathrm{mBar})\end{array}$ & $\begin{array}{l}\text { Wind } \\
\text { Speed } \\
(\mathrm{km} / \mathrm{h})\end{array}$ & $\begin{array}{l}\text { Wind } \\
\text { Dir. }\end{array}$ & Comments \\
\hline \multirow[t]{7}{*}{354} & $11: 09$ & 45450 & -22 & 743.34 & 742.64 & -15 & NNW & \multirow[t]{7}{*}{ clear and sunny } \\
\hline & $13: 54$ & 48750 & -18 & 742.28 & 741.60 & $\sim 20$ & NW & \\
\hline & $15: 21$ & 50700 & -17 & 741.75 & 741.14 & -22 & $w$ & \\
\hline & $16: 35$ & 52800 & -17 & 742.16 & 741.50 & $\sim 24$ & NW & \\
\hline & $17: 35$ & 54600 & -16 & 743.06 & 742.32 & $\sim 18$ & NNW & \\
\hline & 19:05 & 56700 & -16.5 & 744.84 & 744.14 & $\sim 13$ & NW & \\
\hline & $20: 22$ & 58800 & -17 & 745.56 & 744.84 & $\sim 6$ & NW & \\
\hline \multirow[t]{6}{*}{355} & $11: 18$ & 57900 & -19 & 748.03 & 747.61 & -3 & NW & \multirow{6}{*}{$\begin{array}{l}\text { total cover of high cloud, not } \\
\text { affecting visibility }\end{array}$} \\
\hline & 13:15 & 60900 & -11 & 747.79 & 747.17 & $\sim 4$ & $N$ & \\
\hline & $14: 20$ & 63150 & -11 & 746.63 & 745.94 & -2 & NW & \\
\hline & $15: 05$ & 64350 & -13 & 746.32 & 745.65 & $\sim 1$ & NW & \\
\hline & $16: 10$ & 65850 & -13 & 746.06 & 745.38 & $\sim 0.65$ & & \\
\hline & $17: 45$ & 67950 & -14 & 745.87 & 745.24 & $\sim 0.5$ & sw & \\
\hline \multirow[t]{3}{*}{356} & $13: 19$ & 69900 & -12.5 & 743.14 & 742.46 & -2 & NW & \multirow{3}{*}{$\begin{array}{l}\text { overcast with light snow } \\
\text { still good visibility }\end{array}$} \\
\hline & $14: 32$ & 71550 & -13.5 & 742.16 & 741.49 & -1 & NW & \\
\hline & 16:05 & 73650 & -13 & 742.19 & 741.28 & -3 & W & \\
\hline 357 & & 74550 & -18 & & & 32 & $\mathrm{~W}$ & clear and sunny with blowing snow \\
\hline 358 & & 74550 & -20 & & & 34 & WSW & clear and sunny with blowing snow \\
\hline 359 & & 74550 & -19 & & & 20 & $W$ & clear and sunny with blowing snow \\
\hline 360 & & 74550 & -18 & & & 35 & $\bar{W}$ & $\begin{array}{l}\text { scattered high cloud, wind blown } \\
\text { snow affecting visibility }\end{array}$ \\
\hline \multirow[t]{9}{*}{361} & $\overline{14: 14}$ & 75750 & -18 & & & 27 & W & \multirow[t]{9}{*}{ clear and sunny } \\
\hline & $15: 05$ & 77850 & -17.5 & & & 23 & w & \\
\hline & $15: 41$ & 79950 & -18 & & & 22 & w & \\
\hline & $16: 08$ & 81150 & -17.5 & & & 26 & WSW & \\
\hline & $16: 37$ & 82500 & -17.5 & & & 24 & WSW & \\
\hline & $17: 12$ & 84600 & -17 & & & 27 & SW & \\
\hline & $17: 47$ & 86700 & -17 & & & 26 & WSW & \\
\hline & $18: 25$ & 88800 & -16 & & & 28 & WSW & \\
\hline & 19:57 & 90500 & & & & & & \\
\hline \multirow[t]{8}{*}{362} & $10: 05$ & 90900 & -20 & 730.49 & 729.78 & 19 & WSW & \multirow{8}{*}{$\begin{array}{l}\text { scattered high cloud, clearing by } \\
\text { afternoon, variable visibility later } \\
\text { in day as low cloud drifted across }\end{array}$} \\
\hline & $11: 02$ & 93000 & -20 & 730.00 & 729.35 & 19 & WSW & \\
\hline & $12: 00$ & 94800 & -20 & 728.98 & 728.28 & 20 & WSW & \\
\hline & $13: 15$ & 96900 & -19 & 729.55 & 728.67 & 18 & SW & \\
\hline & $14: 54$ & 99000 & -17 & 729.90 & 729.06 & 16 & sw & \\
\hline & $16: 08$ & 101100 & -18 & 729.78 & 728.81 & 18 & sw & \\
\hline & $17: 15$ & 103200 & -18.5 & 730.04 & 729.29 & 15 & s & \\
\hline & $18: 25$ & 105300 & -18 & 730.19 & 729.28 & 15 & s & \\
\hline 363 & & 105300 & -19 & & & 40 & SW & $\begin{array}{l}\text { clear and sunny, reduced visibility } \\
\text { due to blowing snow }\end{array}$ \\
\hline 364 & & 105300 & -18 & & & 42 & SW & same \\
\hline 365 & & 105300 & -20 & & & 30 & SW & same \\
\hline
\end{tabular}




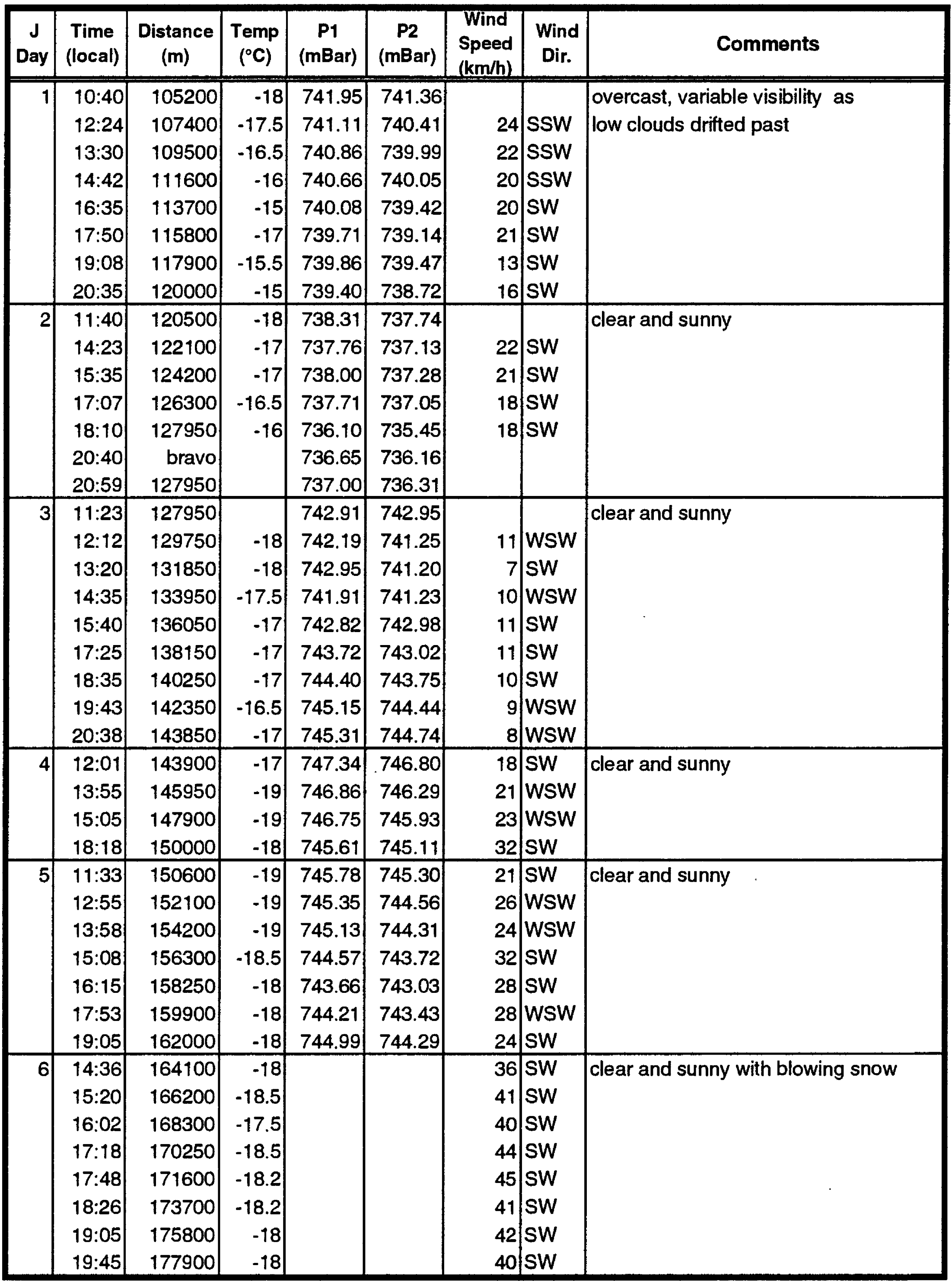




\begin{tabular}{|c|c|c|c|c|c|c|c|c|}
\hline$\underset{\text { Day }}{J}$ & $\begin{array}{c}\text { Time } \\
\text { (local) }\end{array}$ & $\begin{array}{l}\text { Distance } \\
\text { (m) }\end{array}$ & $\begin{array}{c}\text { Temp } \\
\left({ }^{\circ} \mathrm{C}\right)\end{array}$ & $\begin{array}{c}\text { P1 } \\
\text { (mBar) }\end{array}$ & $\begin{array}{c}\text { P2 } \\
\text { (mBar) }\end{array}$ & $\begin{array}{l}\text { Wind } \\
\text { Speed } \\
(\mathrm{km} / \mathrm{h})\end{array}$ & $\begin{array}{l}\text { Wind } \\
\text { Dir. }\end{array}$ & Comments \\
\hline & $20: 29$ & 179850 & -18 & & & 40 & SW & \\
\hline & $21: 25$ & 181950 & -17 & & & 39 & sw & \\
\hline & 22:25 & 183900 & -15.5 & & & 37 & sw & \\
\hline 7 & & 185400 & -19 & & & 30 & WSW & $\begin{array}{l}\text { clear \& sunny, w/reduced visibility } \\
(<1 \mathrm{~km}) \text { due to blowing snow }\end{array}$ \\
\hline \multirow[t]{5}{*}{8} & $13: 50$ & 187500 & -19 & 735.87 & 735.17 & 17 & WSW & \multirow{5}{*}{$\begin{array}{l}\text { overcast, but good visibility. By } \\
\text { afternoon, visibility variable due } \\
\text { to low clouds drifting across }\end{array}$} \\
\hline & $14: 58$ & 188700 & -20 & 735.11 & 734.34 & 18 & W & \\
\hline & $16: 48$ & 190800 & -19 & 734.16 & 733.37 & 16 & $\mathrm{~W}$ & \\
\hline & $18: 38$ & 192900 & -18 & 733.21 & 732.50 & 17 & W & \\
\hline & 20:10 & 195000 & -18 & 733.01 & 732.36 & 23 & sw & \\
\hline \multirow[t]{6}{*}{9} & $11: 40$ & 195300 & -18 & 733.41 & 732.70 & 9 & SW & \multirow{6}{*}{$\begin{array}{l}\text { scattered high clouds. By afternoon } \\
\text { visibility variable due to low clouds } \\
\text { drifting across. }\end{array}$} \\
\hline & $13: 21$ & 197100 & -21 & 732.89 & 732.19 & 15 & WSW & \\
\hline & $14: 40$ & 199200 & -19.5 & 732.74 & 732.07 & 16 & sw & \\
\hline & $16: 45$ & 201300 & -18 & 732.29 & 731.49 & 16 & sw & \\
\hline & $18: 04$ & 203400 & -18 & 731.76 & 731.10 & 12 & sw & \\
\hline & $20: 12$ & 205500 & -18 & 731.75 & 731.23 & 11 & SW & \\
\hline \multirow[t]{5}{*}{10} & $13: 40$ & 207600 & -20 & 728.79 & 727.41 & 16 & SW & \multirow[t]{5}{*}{ overcast but good visibility } \\
\hline & $14: 58$ & 209550 & -19 & 728.44 & 727.82 & 15 & SW & \\
\hline & $16: 20$ & 211500 & -17 & 728.06 & 727.41 & 15 & sw & \\
\hline & $18: 26$ & 213900 & -16 & 727.72 & 726.96 & 12 & Wsw & \\
\hline & 19:54 & 216000 & -17 & 728.02 & 727.13 & 19 & SW & \\
\hline 11 & & 216000 & -17 & & & 15 & SW & $\begin{array}{l}\text { overcast with occ. light snow. Poor } \\
\text { visibility }(<1 \mathrm{~km}) \text { and surface def. }\end{array}$ \\
\hline \multirow[t]{5}{*}{12} & $13: 12$ & 218100 & -19.5 & 729.30 & 728.34 & 20 & WSW & \multirow{5}{*}{$\begin{array}{l}\text { morning: variable visibility due to low } \\
\text { cloud drifting across. } \\
\text { Clear by afternoon }\end{array}$} \\
\hline & $14: 35$ & 220200 & -19.5 & 728.76 & 728.05 & 26 & WSW & \\
\hline & $16: 10$ & 222300 & -20 & 728.43 & 727.84 & 20 & W & \\
\hline & $17: 25$ & 224400 & -20 & 727.89 & 727.06 & 22 & SW & \\
\hline & 19:15 & 226500 & -19 & 728.03 & 727.34 & 18 & sw & \\
\hline \multirow[t]{5}{*}{13} & $11: 30$ & 227700 & -21 & 727.85 & 727.24 & 10 & S & \multirow{5}{*}{$\begin{array}{l}\text { overcast, occ. flurries of light snow } \\
\text { good visibility, but no surface def. }\end{array}$} \\
\hline & $15: 35$ & 228600 & -17 & 727.95 & 727.33 & 8 & NE & \\
\hline & $16: 48$ & 230700 & -20 & 727.50 & 726.98 & 13 & ENE & \\
\hline & $18: 15$ & 232800 & -19 & 727.43 & 726.82 & o) & & \\
\hline & $19: 40$ & 234900 & -19 & 727.30 & 726.51 & 2 & ESE & \\
\hline 14 & & 235400 & -21 & & & 17 & SW & scattered high cloud. good visibility \\
\hline 15 & & 235400 & -20 & & & 12 & $S$ & restricted vis. and def. due to low cloud \\
\hline \multirow[t]{3}{*}{16} & $16: 08$ & 237000 & -20 & 729.29 & 728.34 & 21 & SW & \multirow[t]{3}{*}{ misty low cloud, visibility to $5 \mathrm{~km}$. } \\
\hline & $17: 23$ & 239100 & -19 & 729.80 & 728.66 & 28 & sw & \\
\hline & $19: 01$ & 241200 & -20 & 729.74 & 729.09 & 26 & sw & \\
\hline 17 & & 241400 & & & & 44 & SW & clear and sunny with blowing snow \\
\hline 18 & & 241400 & -19 & & & 37 & SW & clear and sunny with blowing snow \\
\hline 19 & & 241400 & -20 & & & 33 & SSW & same, with low cloud late in day \\
\hline \multirow[t]{2}{*}{20} & $14: 12$ & 243300 & -23 & 723.08 & 722.45 & 30 & sw & \multirow{2}{*}{$\begin{array}{l}\text { clear \& sunny w/ blowing snow early } \\
\text { disappeared late as wind died }\end{array}$} \\
\hline & $16: 42$ & 245400 & -23 & 722.45 & 721.58 & 31 & SW & \\
\hline
\end{tabular}




\begin{tabular}{|c|c|c|c|c|c|c|c|c|}
\hline $\begin{array}{c}J \\
\text { Day }\end{array}$ & $\begin{array}{c}\text { Time } \\
\text { (local) }\end{array}$ & $\begin{array}{c}\text { Distance } \\
(\mathrm{m})\end{array}$ & $\begin{array}{c}\text { Temp } \\
\left({ }^{\circ} \mathrm{C}\right)\end{array}$ & $\begin{array}{c}\text { P1 } \\
(\mathrm{mBar})\end{array}$ & $\begin{array}{c}\text { P2 } \\
\text { (mBar) }\end{array}$ & $\begin{array}{l}\text { Wind } \\
\text { Speed } \\
(\mathbf{k m} / \mathbf{h})\end{array}$ & $\begin{array}{l}\text { Wind } \\
\text { Dir. }\end{array}$ & Comments \\
\hline & $\begin{array}{l}18: 23 \\
20: 40 \\
22: 28 \\
23: 59 \\
\end{array}$ & $\begin{array}{l}247800 \\
249900 \\
252000 \\
254100 \\
\end{array}$ & $\begin{array}{r}-21 \\
-22 \\
-23 \\
-23.5 \\
\end{array}$ & & $\begin{array}{l}721.91 \\
720.63 \\
720.60 \\
720.20 \\
\end{array}$ & $\begin{array}{c}23 \\
16 \\
10 \\
10 \\
\end{array}$ & $\begin{array}{l}\text { SW } \\
\text { SW } \\
\text { SW } \\
\text { SW }\end{array}$ & . \\
\hline 21 & $\begin{array}{l}20: 20 \\
22: 00\end{array}$ & $\begin{array}{r}257860 \\
\text { charlie }\end{array}$ & & & $\begin{array}{l}721.23 \\
721.10 \\
\end{array}$ & & & scattered high cloud \\
\hline 23 & $\begin{array}{r}7: 09 \\
7: 50 \\
8: 05 \\
9: 05 \\
9: 50 \\
10: 25 \\
11: 05 \\
11: 50 \\
12: 35 \\
18: 18 \\
19: 35 \\
20: 26 \\
20: 55 \\
21: 18 \\
21: 41 \\
22: 06 \\
22: 31 \\
22: 55 \\
23: 30 \\
23: 59 \\
\end{array}$ & $\begin{array}{l}256200 \\
257860 \\
258300 \\
260400 \\
262500 \\
264300 \\
266100 \\
268200 \\
270300 \\
269700 \\
272400 \\
274500 \\
276600 \\
278700 \\
280800 \\
282900 \\
285000 \\
287100 \\
289200 \\
291300 \\
\end{array}$ & $\begin{array}{r}-26 \\
-20 \\
-24 \\
-22 \\
-22 \\
-22 \\
-21 \\
-23 \\
-22 \\
\\
-20 \\
-21 \\
-21 \\
-21 \\
-22 \\
-21.5 \\
-21 \\
-22 \\
-24 \\
-21.5 \\
\end{array}$ & $\begin{array}{l}720.82 \\
721.53 \\
721.72 \\
722.07 \\
721.84 \\
721.87 \\
721.89 \\
721.79 \\
721.84 \\
722.17 \\
722.04\end{array}$ & $\begin{array}{l}720.82 \\
720.78 \\
720.79 \\
720.67 \\
720.70 \\
720.60 \\
720.24 \\
719.91 \\
719.93 \\
720.66 \\
720.96 \\
721.17 \\
721.08 \\
721.23 \\
721.04 \\
720.90 \\
721.06 \\
721.36 \\
721.27 \\
\end{array}$ & $\begin{array}{l}10 \\
22 \\
21 \\
21 \\
22 \\
18 \\
22 \\
21 \\
17 \\
16 \\
\end{array}$ & $\begin{array}{l}\text { SW } \\
\text { S } \\
\text { S } \\
\text { S } \\
S \\
S \\
S \\
S \\
\text { S } \\
\text { SSW } \\
\end{array}$ & mostly overcast, occ. light snow flurry \\
\hline 24 & $\begin{array}{l}0: 20 \\
0: 43 \\
1: 29 \\
1: 54 \\
2: 18 \\
2: 46 \\
3: 09 \\
3: 35 \\
3: 57 \\
4: 27 \\
\end{array}$ & $\begin{array}{l}293400 \\
295500 \\
297600 \\
299700 \\
301800 \\
303900 \\
306000 \\
308100 \\
310200 \\
312600 \\
\end{array}$ & $\begin{array}{l}-21 \\
-20 \\
-22 \\
-23 \\
-25 \\
-23 \\
-24 \\
-24 \\
-28 \\
-29 \\
\end{array}$ & $\begin{array}{l}722.01 \\
721.90 \\
722.02 \\
722.26 \\
722.16 \\
721.87 \\
721.41 \\
721.30 \\
721.26 \\
721.55 \\
\end{array}$ & $\begin{array}{l}721.20 \\
721.22 \\
721.07 \\
721.46 \\
721.27 \\
720.84 \\
720.66 \\
720.47 \\
720.36 \\
720.68 \\
\end{array}$ & $\begin{array}{r}12 \\
12 \\
11 \\
10 \\
10 \\
12 \\
10 \\
10 \\
10 \\
9 \\
\end{array}$ & $\begin{array}{l}\text { S } \\
\text { SW } \\
\text { SSW } \\
\text { SSW } \\
\text { SSW } \\
\text { SSW } \\
\text { SSW } \\
\text { SSW } \\
\text { SSW } \\
\text { SSW } \\
\end{array}$ & $\begin{array}{l}\text { scattered high cloud, occ. low clouds } \\
\text { drifting across }\end{array}$ \\
\hline 25 & & 269700 & & & & & & $\begin{array}{l}\text { hazy, low cloud, clearing as day } \\
\text { progressed }\end{array}$ \\
\hline
\end{tabular}



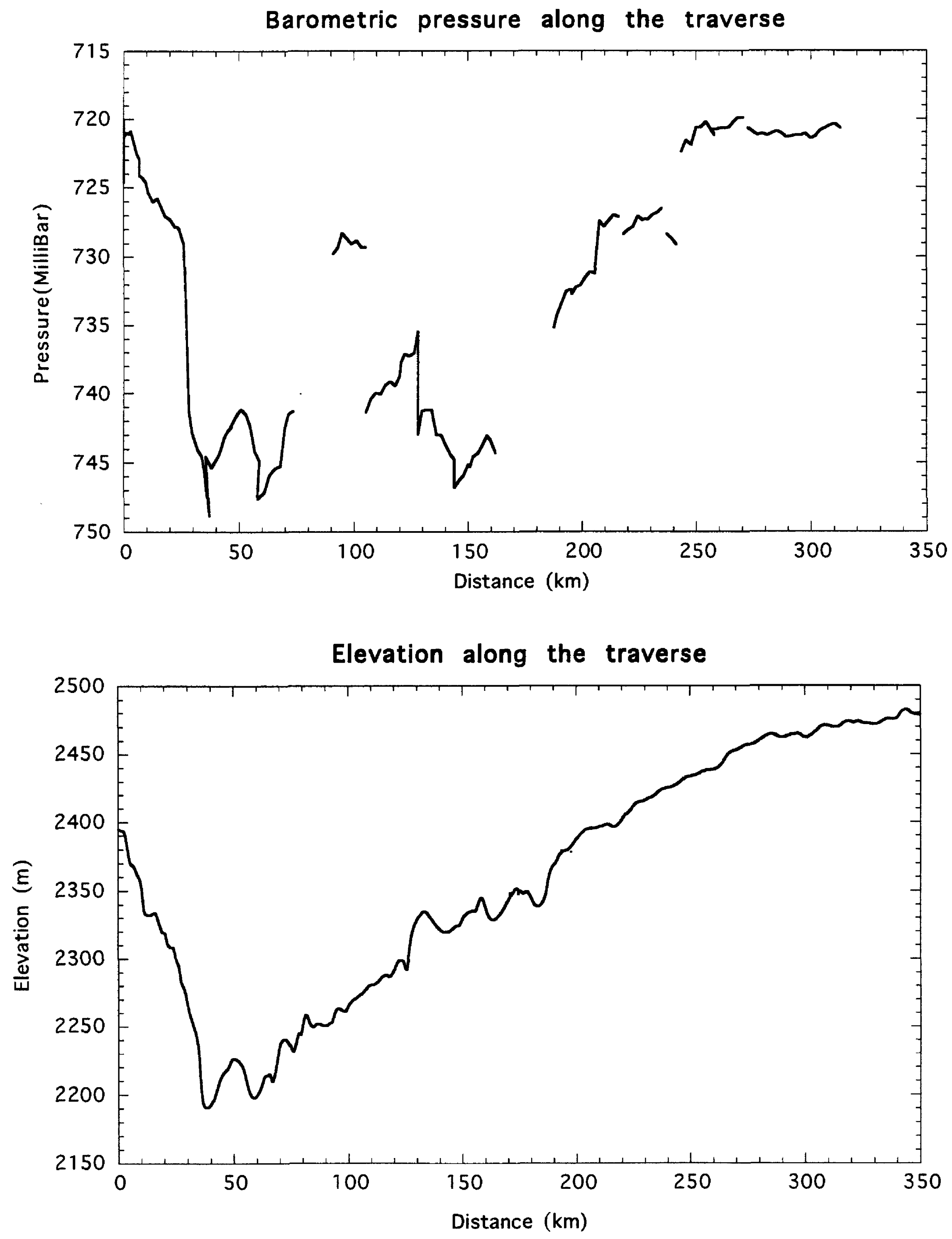

Figure A5.1 Top - barometric pressure (in milliBar) measured along the traverse line. Bottom - elevation along the traverse (similar to Figure 4). Note that pressure is plotted increasing downward to facilitate comparison with the elevation. 
APPENDIX 6: Weather Observations by the Advance Party

by Bill King

\begin{tabular}{|c|c|c|c|c|c|c|}
\hline Day| & $\begin{array}{c}\text { Time } \\
\text { (local) }\end{array}$ & $\begin{array}{l}\text { Distance } \\
\text { (m) }\end{array}$ & $\begin{array}{l}\text { Temp. } \\
\left({ }^{\circ} \mathrm{C}\right)\end{array}$ & $\begin{array}{l}\text { Wind } \\
\text { Speed } \\
\text { (knot) }\end{array}$ & $\begin{array}{l}\text { Wind } \\
\text { Dir. }\end{array}$ & Comments \\
\hline 336 & 930 & 0 & -31 & 9 & 230 & \\
\hline 336 & 1700 & 0 & -27 & 13 & 230 & Slight haze towards horizon. \\
\hline 337 & 930 & 0 & -30 & 0 & 0 & \\
\hline 337 & 2100 & 9600 & -22 & o) & 0 & Low cloud bands towards Portal Mt. \\
\hline 338 & 930 & 9600 & -27 & 0 & 0 & \\
\hline 338 & 2100 & 24000 & -26 & 0 & 0 & Fog bands east towards edge of polar plateau. \\
\hline 339 & 930 & 24000 & -31 & 9 & 320 & \\
\hline 339 & 2100 & 41100 & -25 & 9 & 320 & 10 knot westward wind all afternoon. \\
\hline 340 & 930 & 41100 & -27 & 13 & 260 & Wind all day. \\
\hline 341 & 930 & 46600 & -27 & 13 & 260 & \\
\hline 341 & 2100 & 58800 & -25 & o & o & Wind most of day. Stopped early evening. \\
\hline 342 & 930 & 63000 & -31 & 9 & 260 & \\
\hline 342 & 2130 & 75700 & -25 & 0 & of & Extremely clear visibility, Possibly $100 \mathrm{~km}$. \\
\hline 343 & 930 & 75700 & -28 & 9 & 260 & \\
\hline 343 & 2230 & 90900 & -23 & 0 & 0 & Clouds in from SW late afternoon. \\
\hline 344 & 930 & 90900 & -25 & 9 & 260 & \\
\hline 345 & 930 & 107400 & -23 & 0 & 0 & \\
\hline 346 & 1400 & 125000 & -24 & 13 & 260 & \\
\hline 347 & 930 & 125000 & -30 & 9 & 260 & \\
\hline 347 & 2200 & 142300 & -25 & 0 & 0 & High stratus mid afternoon. \\
\hline 348 & 930 & 142300 & -26 & 13 & 260 & Fog on horizon. \\
\hline 348 & 2130 & 154200 & -25 & 15 & 230 & Minor fog on western horizon, wind all day. \\
\hline 349 & 930 & 154200 & -28 & 13 & 230 & \\
\hline 350 & 930 & 168300 & -27 & 24 & 230 & \\
\hline 351 & 930 & 171600 & -23 & 9 & 230 & \\
\hline 351 & 2200 & 185400 & -20 & 20 & 160 & \\
\hline 352 & 1200 & 185400 & -18 & 10 & 180 & \\
\hline 353 & 930 & 185400 & -19 & 9 & 230 & \\
\hline 354 & 930 & 185400 & -23 & 9 & 260 & \\
\hline 354 & 2300 & 199200 & -21 & 5 & 260 & Fog on horizon, drifting snow mid afternoon. \\
\hline 355 & 930 & 199200 & -24 & 0 & 0 & \\
\hline 355 & 2200 & 213900 & -20 & 0 & 0 & Horizons foggy, western horizon foggy. \\
\hline 356 & 930 & 213900 & -22 & 9 & 240 & \\
\hline 356 & 2200 & 228600 & -23 & 11 & 260 & \\
\hline 357 & 1300 & 228600 & -22 & 19 & 260 & \\
\hline 358 & 930 & 228600 & -23 & 30 & 260 & Visibility variable from $4 \mathrm{~km}$ to $20 \mathrm{~km}$. \\
\hline 359 & 930 & 228600 & -24 & 13 & 260 & \\
\hline 360 & 1300 & 245400 & -22 & 30) & 260 & \\
\hline 361 & 930 & 245400 & -24 & 15 & 260 & \\
\hline 362 & 930 & 245400 & -25 & 9 & 260 & Halo around sun. \\
\hline 362 & 2330 & 260400 & -23 & 15 & 230 & \\
\hline 363 & 1200 & 260400 & -23 & 24 & 230 & Wind increased late afternoon to over 30 knots. \\
\hline 364 & 930 & 260400 & -23 & 35 & 250 & Large amounts of drifting snow. \\
\hline
\end{tabular}




\begin{tabular}{|r|r|r|r|r|r|l|}
\hline$J$ Day & $\begin{array}{r}\text { Time } \\
(\text { local })\end{array}$ & $\begin{array}{r}\text { Distance } \\
(\mathbf{m})\end{array}$ & $\begin{array}{c}\text { Temp. } \\
\left({ }^{\circ} \mathbf{C}\right)\end{array}$ & $\begin{array}{c}\text { Wind } \\
\text { Speed } \\
(\mathbf{k n o t})\end{array}$ & $\begin{array}{c}\text { Wind } \\
\text { Dir. }\end{array}$ & \multicolumn{2}{|l|}{ Comments } \\
\hline \hline 365 & 930 & 260400 & -22 & 30 & 250 & \\
1 & 930 & 260400 & -24 & 19 & 250 & Fog on west afternoon \\
2 & 930 & 260400 & -24 & 19 & 190 & \\
2 & 2200 & 285000 & -20 & 9 & 190 & \\
3 & 930 & 285000 & -23 & 5 & 190 & \\
4 & 930 & 285000 & -23 & 13 & 210 & Wind increasing \\
4 & 2000 & 312600 & -19 & 24 & 210 & \\
5 & 930 & 312600 & -24 & 13 & 210 & \\
5 & 2300 & 342000 & -21 & 13 & 250 & \\
6 & 930 & 342000 & -24 & 24 & 250 & Strong wind all day. \\
6 & 2200 & 360900 & -19 & 30 & 250 & \\
7 & 930 & 360900 & -23 & 30 & 250 & Poor ground definition. \\
8 & 930 & 312500 & -20 & 13 & 250 & \\
\hline
\end{tabular}

NOTE: Wind speed was estimated. Comparison with measured wind speed by main party (Appendix 5 and Figure 11 ) indicates actual speed to be $\sim 65 \%$ of that estimated. 
APPENDIX 7: Barometric Pressure and Temperatures at the Temporary Base Camp Averaged Over 6 Hours

by Rebecca Drury and Ron Hackney

\begin{tabular}{|r|r|r|r|}
\hline J day & $\begin{array}{r}\text { Barometric } \\
\text { Press. (mBar) }\end{array}$ & $\begin{array}{r}\text { Distance } \\
(\mathbf{m})\end{array}$ & $\begin{array}{r}\text { Temp. } \\
\left({ }^{\circ} \mathrm{C}\right)\end{array}$ \\
\hline 344.00 & 719.08 & 0 & -18 \\
344.25 & 718.60 & 0 & -21 \\
344.50 & 718.78 & 0 & -18 \\
344.75 & 720.10 & 0 & -20 \\
345.00 & 721.83 & 0 & -24 \\
345.25 & 722.60 & 0 & -24 \\
345.50 & 722.90 & 0 & -25 \\
345.75 & 722.08 & 0 & -27 \\
346.00 & 720.37 & 0 & -32 \\
346.25 & 719.00 & 0 & -30 \\
346.50 & 718.95 & 0 & -24 \\
346.75 & 721.64 & 0 & \\
347.00 & 722.10 & 6900 & -33 \\
347.25 & 722.20 & 6900 & -29 \\
347.50 & 722.30 & 6900 & -22 \\
347.75 & 725.13 & 6900 & \\
348.00 & 724.70 & 14700 & -28 \\
348.25 & 724.23 & 14700 & -26 \\
348.50 & 724.10 & 14700 & -25 \\
348.75 & 725.30 & 14700 & -17 \\
349.00 & 725.90 & 19650 & -26 \\
349.25 & 725.50 & 19650 & -27 \\
349.50 & 725.30 & 19650 & -23 \\
349.75 & 726.50 & 19650 & -26 \\
350.00 & 726.47 & 24300 & -29 \\
350.25 & 728.08 & 24300 & -26 \\
350.50 & 731.21 & 24300 & -24 \\
350.75 & 733.95 & 24300 & -24 \\
351.00 & 737.02 & 24300 & -28 \\
351.25 & 738.68 & 24300 & -25 \\
351.50 & 738.00 & 24300 & -15 \\
351.75 & 744.50 & 24300 & -15 \\
352.00 & 743.50 & 35450 & -18 \\
352.25 & 741.94 & 35450 & -19 \\
352.50 & 740.88 & 35450 & -18 \\
352.75 & 741.46 & 35450 & -19 \\
353.00 & 742.10 & 35450 & -20 \\
353.25 & 742.61 & 35450 & -17 \\
353.50 & 742.62 & 35450 & -13 \\
353.75 & 742.26 & 35450 & \\
354.00 & 741.32 & 45450 & \\
354.25 & 741.00 & 45450 & \\
354.50 & 740.90 & 45450 & \\
354.75 & 741.41 & 45450 & \\
355.00 & 744.31 & 57900 & \\
355.25 & 745.40 & 57900 & \\
355.50 & 745.96 & 57900 & \\
355.75 & 744.14 & 57900 & \\
\hline & & & \\
\hline
\end{tabular}

\begin{tabular}{|r|r|r|}
\hline $\mathrm{J}$ day & $\begin{array}{r}\text { Barometric } \\
\text { Press. (mBar) }\end{array}$ & $\begin{array}{c}\text { Distance } \\
(\mathbf{m})\end{array}$ \\
\hline \hline 356.00 & 743.30 & 69000 \\
\hline 356.25 & 742.60 & 69000 \\
356.50 & 741.90 & 69000 \\
356.75 & 740.80 & 69000 \\
357.00 & 739.65 & 74550 \\
357.25 & 738.49 & 74550 \\
357.50 & 737.56 & 74550 \\
357.75 & 737.66 & 74550 \\
358.00 & 738.20 & 74550 \\
358.25 & 738.24 & 74550 \\
358.50 & 739.04 & 74550 \\
358.75 & 739.40 & 74550 \\
359.00 & 738.86 & 74550 \\
359.25 & 738.74 & 74550 \\
359.50 & 738.90 & 74550 \\
359.75 & 739.00 & 74550 \\
360.00 & 738.78 & 74550 \\
360.25 & 737.32 & 74550 \\
\hline 360.50 & 736.82 & 74550 \\
360.75 & 737.29 & 74550 \\
361.00 & 735.88 & 74550 \\
362.00 & 728.22 & 90300 \\
362.25 & 727.78 & 90300 \\
362.50 & 728.80 & 90300 \\
362.75 & 729.47 & 90300 \\
\hline 363.00 & 730.60 & 105300 \\
363.25 & 731.52 & 105300 \\
\hline 363.50 & 732.91 & 105300 \\
363.75 & 734.97 & 105300 \\
364.00 & 736.80 & 105300 \\
364.25 & 737.52 & 105300 \\
364.50 & 737.54 & 105300 \\
364.75 & 738.51 & 105300 \\
365.00 & 737.95 & 105300 \\
\hline 365.25 & 737.99 & 105300 \\
365.50 & 738.50 & 105300 \\
365.75 & 738.90 & 105300 \\
1.00 & 739.80 & 105300 \\
1.25 & 739.40 & 105300 \\
1.50 & 739.09 & 105300 \\
\hline 1.75 & 737.88 & 105300 \\
2.00 & 736.70 & 120450 \\
2.25 & 736.10 & 120450 \\
2.50 & 736.10 & 120450 \\
3.75 & 735.80 & 120450 \\
3.25 & 737.08 & 127700 \\
\hline 3.50 & 739.52 & 127700 \\
\hline & 742.13 & 127700 \\
\hline
\end{tabular}




\begin{tabular}{|r|r|r|}
\hline $\mathrm{J}$ day & $\begin{array}{c}\text { Barometric } \\
\text { Press. (mBar) }\end{array}$ & $\begin{array}{c}\text { Distance } \\
(\mathrm{m})\end{array}$ \\
\hline \hline 3.75 & 743.10 & 127700 \\
4.00 & 744.80 & 143850 \\
4.25 & 744.98 & 143850 \\
4.50 & 744.85 & 143850 \\
4.75 & 744.50 & 143850 \\
5.00 & 743.70 & 150600 \\
5.25 & 743.50 & 150600 \\
5.50 & 743.30 & 150600 \\
5.75 & 742.70 & 150600 \\
6.00 & 742.10 & 161850 \\
6.25 & 741.24 & 161850 \\
7.25 & 735.59 & 185400 \\
7.50 & 733.83 & 185400 \\
7.75 & 733.73 & 185400 \\
8.00 & 733.76 & 185400 \\
8.25 & 735.08 & 185400 \\
8.50 & 735.35 & 185400 \\
8.75 & 732.03 & 185400 \\
9.00 & 730.22 & 195300 \\
9.25 & 730.26 & 195300 \\
9.50 & 731.14 & 195300 \\
9.75 & 729.95 & 195300 \\
10.00 & 728.40 & 205400 \\
10.25 & 727.06 & 205400 \\
10.50 & 726.24 & 205400 \\
10.75 & 725.29 & 205400 \\
11.00 & 725.10 & 216000 \\
11.25 & 725.06 & 216000 \\
11.50 & 726.20 & 216000 \\
11.75 & 727.70 & 216000 \\
12.00 & 728.30 & 216000 \\
12.25 & 727.96 & 216000 \\
12.50 & 727.40 & 216000 \\
12.75 & 726.90 & 216000 \\
13.00 & 725.64 & 227600 \\
13.25 & 725.50 & 227600 \\
13.50 & 725.74 & 227600 \\
13.75 & 725.70 & 227600 \\
14.00 & 724.98 & 235400 \\
14.25 & 724.72 & 235400 \\
14.50 & 724.66 & 235400 \\
14.75 & 724.06 & 235400 \\
15.00 & 724.98 & 235400 \\
15.25 & 724.72 & 235400 \\
\hline & & \\
\hline
\end{tabular}

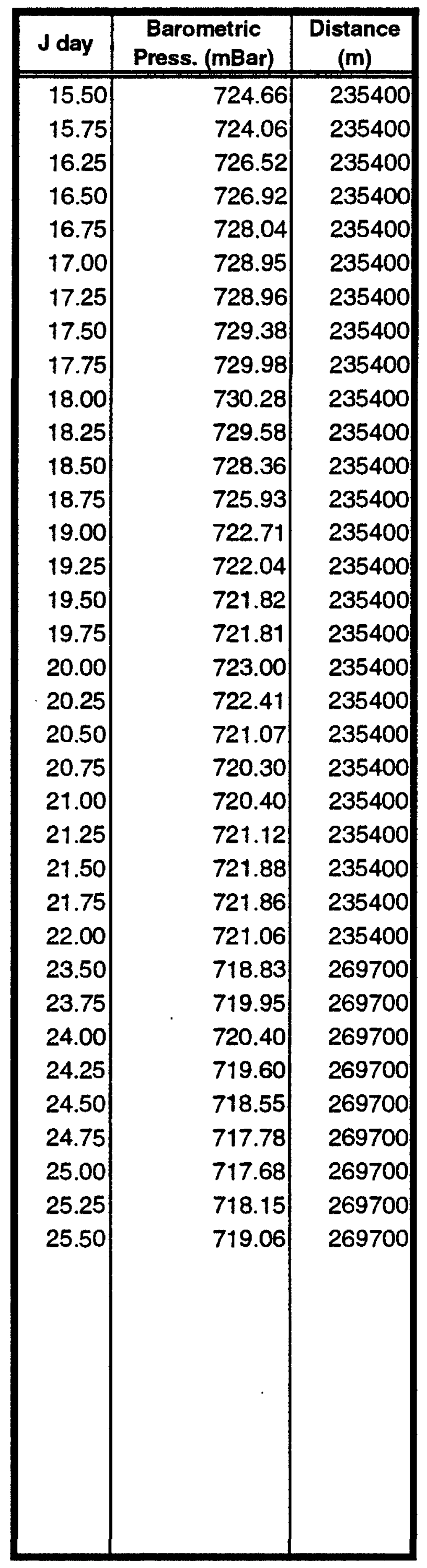




\title{
APPENDIX 8 : Mechanic's Report
}

\section{General Comments}

\author{
By Mike Collins
}

\section{Project Equipment}

United States.

Plant

Number Use

Snocat Tucker

069

Snocat Tucker

071

Sledge, 1 Ton

Sledge, ASV

Generator, Onan $3.5 \mathrm{~kW}$

Generator, Onan $3.5 \mathrm{~kW}$

3

10

Wannagan and general

Explosives and plowing

General use to $\mathrm{km} 75$

Not used

Vehicle heating

New Zealand.

Hagglund

Skidoo Alpine II

Ski-doo Alpine II

Ski-doo Alpine II

Ski-doo Alpine II

Sledge, Maudheim

Sledge, Maudheim

Sledge, Anare

Sledge, Anare

Generator, Yanmar $3.0 \mathrm{~kW}$

Generator, Yanmar $3.0 \mathrm{~kW}$

Generator, Yanmar $3.0 \mathrm{~kW}$

Generator, Honda $2.5 \mathrm{~kW}$

Generator, Honda $5.0 \mathrm{~kW}$
$\mathrm{H} 28$

AL3

AL4

AL5

AL7

FG8

FG7

FG1

PG5

PG15
Seismic recording system

Detonator sledge

Radar

Survey party

Survey party

Fuel, generators, Herman Nelson

General use

Wannagan (living quarters)

Explosives and fuel

Heating and battery charging

Heating and battery charging

Heating and battery charging

Heating and battery charging

Heating and battery charging

\section{Preparation}

I was given only two weeks to prepare the vehicles. It was not sufficient time to carry out the work, but with the help of Jeremy Ridgin and Gus McAlister, the deadline was met.

The 4 Skidoos needed quite a lot of work to make them functional. Examples of problems were: leaking shock absorber, suspension springs broken, hinges broken away from bonnet.

Skidoo AL7 was also modified with a 50 Ampere-hour (Ah) dry battery and fitted with a charger set for recharging VHF radio batteries. An ignition primary circuit amplifier was fitted, because this machine had a Dugati ignition system. The ignition system was needed to enhance engine starting temperatures below $-25^{\circ} \mathrm{C}$. 170 jets were fitted to all machines. This was just on the rich side for the $8000 \mathrm{ft}$ elevation but proved satisfactory (160 jets were carried). The Hagglund was serviced by Gus McAlister, along with some other minor checks. 
The 069 Tucker was serviced by the Heavy Shop in McMurdo, and new track belting was fitted all round. 071 Tucker had already been transported to Taylor Dome in October and had been working up there since that time. This vehicle, too, was suffering track belting problems, so was fitted with new track belting all round before we arrived at Taylor Dome in early December.

The other major part of the preparation was organizing spare parts, tools, and the necessary oil for the vehicles used by both the advance and the main parties.

\section{Operating conditions}

The temperature ranged between $-30^{\circ} \mathrm{C}$ and $-10^{\circ} \mathrm{C}$. Starting problems were anticipated. The direct drive petrol generators (PG15 and Onans) could be started unaided, whereas the Yanmar Diesel units, and PG5 (which had a belt drive) needed to be preheated with the Herman Nelson for 5-10 minutes and then could be started easily. The Hagglund's coolant heater was plugged in for approximately 1 hour most days before starting. This was not always necessary, but reduced the strain on the starting systems that were doing up to 100 stop/starts in a days operation. The Tuckers were our most difficult machine to start. Depending on the temperature they took 1 to 3 hours to start. Our usual approach was to plug the vehicle in for 3 hours, putting the Herman Nelson on the engine compartment for the last 30 minutes of that time. This system worked without fail no matter what the temperature. Although the starting time of the Tuckers could be shortened by not plugging them first to a generator, plugging them made for an easier start. The Herman Nelson hose was tied to an opening in the cowling so the heating took place with the bonnet down. The bonnet grating and grill were also covered to reduce heat loses and to indirectly heat other components, such as the power steer pump and reservoir, V-belts, intake system ducting, and starter motor.

The Skidoos gave no starting problems. The thermal gain under the Ski-doo cover quite often brought the temperature up above $0^{\circ} \mathrm{C}$, so if the machine was started within a few minutes of removing the cover it very rarely had to start at ambient temperature.

\section{Fuel Consumption}

Fuel consumption was well predicted, except for the Tuckers. The 071 Tucker did a lot of stop/start work laying the explosives. Idling time also caused the operating hours and total fuel usage to be higher than expected. This was due mostly to the fact that the Tuckers could not be left shut down for longer than 80 minutes without needing preheating to start again. Finally, the Tuckers could be operated only in first gear and at slow speeds $(1-4 \mathrm{~km} / \mathrm{h})$ because of the rough terrain.

\section{Operators}

We experienced few problems with vehicle operators. Because people seldom swapped tasks, the person driving a vehicle gained experience and a feel for the machine. This may have caused boredom for some, but I believe it reduced operation problems overall.

\section{Tools}

The field tool box, bought in late 1992, and supplemented with some additions for this project, proved a valuable base tool kit. The box has no permanent AF tool sets, and it might be useful to include it in the future. The vice fitted to one of the Anare sledges proved a very useful addition on a number of occasions. 


\title{
Vehicle Maintenance
}

\author{
USAP EQUIPMENT
}

Snocat Tucker 069 pax

Hour meter Start 3535

Finish 3668

Total 133 hours

Fuel Consumption: 2 Liters/km

Maintenance

I replaced primary fuel filter at $3568 \mathrm{hr}$, greased vehicle at $3600 \mathrm{hr}$, replaced transmission filter at $3610 \mathrm{hr}$, removed transmission and installed overhauled unit (twice) at $3644 \mathrm{hr}$. The overhauled transmission failed at $3668 \mathrm{hr}(\mathrm{km} \mathrm{270})$. This Tucker was used to pull the Wannagan and the majority of the fuel carried between supply dumps. It had ample horsepower and traction to pull the load.

\section{Snocat Tucker 071 pax}

Hour meter Start 2888

Fuel Consumption: 2 Liters/km

Finish $3218 \quad$ Total 330 Hours

\section{Maintenance}

Transfer case anchor bolts (8) became lose and were tightened. I greased the machine at $3050 \mathrm{hr}$. Transfer case input flange seal started leaking, so a check on the oil level was made every second day. The front axle steering pivot plate at the rear spring anchor positions was bent. The bent pivot plate indicates that the undercarriage is a little light and prone to damage when pulling this weight under these surface conditions. We avoided further damage by working only when light conditions were suitable to distinguish sastrugi. Operating speed did not contribute to the damage because all travel over this terrain was done in first gear. Tucker 071 was used to pull and lay the explosives. Its operating hours were the greatest of all vehicles, and it performed very well.

\section{Generators: Onan 3.5 kW Units 3 \&10}

Fuel Consumption: 2.5 Liters/Hour

Both these generators ran very well with minimal problems. We experienced some carburetor icing, and overcame it with better positioning of the generator in relation to the prevailing wind. This positioning (similar to a polar tent door in relation to wind) as well as covering the generators on three sides, leaving the exhaust side open also helped its operation. During storms, however, positioning and three-sided covering still did not offer sufficient protection, and the generator quit due to the increased moisture. The oil was changed in both engines once.

\section{Herman Nelson}

Hour meter Start $852 \quad$ Finish $902 \quad$ Total 50 Hours

This machine operated very reliably with only a replacement spark plug needed. The engine/transmission oil was changed once.

\section{NZAP EQUIPMENT}

\section{Hagglund $\mathbf{H} 28$}

Hour meter Start 1089

Odometer Start 7404

Finish 1316

Finish 7761
Total 227

Total $357 \mathrm{Km}$ 
Fuel Consumption: 1 Liter/Km

\section{Maintenance}

The only problem encountered was that the general power supply solenoid jammed in position, so that engine shut down using the key was impossible. This problem only occurred when the temperature was below $-30^{\circ} \mathrm{C}$. The problem could be overcome by heating the cab interior before starting. (A warm cab interior is usual at Scott Base so this problem would normally not occur). A no-start on one occasion was caused by the HF radio draining one of the $12 \mathrm{~V}$ starting batteries. This occurred when we had been stalled for a number of days due to bad weather. The Hagglund towed the streamer and the rear car housed the seismic recording equipment, battery charger's, and VHF radio battery chargers. The start-stop operation is not suited to the turbo-Diesel engine, because it has to idle for at least 2 minutes before shut-down. However,due to a number of other concurrent operations during each shot, this limitation did not seem to affect the daily progress.

\section{Alpine ski-doo AL3}

Hour meter Start 90

Odometer Start 2395

Finish 136

Finish 2771
Total 46 Hours

Total $376 \mathrm{Km}$

Fuel Consumption: $3 \mathrm{Km} / \mathrm{Liter}$.

\section{Maintenance}

The front ski shaft foot broke at $\mathrm{km} \mathrm{208.} \mathrm{The} \mathrm{break} \mathrm{occurred} \mathrm{on} \mathrm{the} \mathrm{right} \mathrm{side} \mathrm{of}$ the middle tract idler brackets. This ski-doo was used to pull the sleepy sledge carrying the detonators. It was replaced by AL7.
Alpine ski-doo AL4
Hour meter Start 75
Finish 172
Total 97 Hours
Odometer Start 4680
Finish 5978
Total $911 \mathrm{Km}$
Fuel Consumption: $3 \mathrm{Km} / \mathrm{Liter}$

\section{Maintenance}

This machine towed the radar, and, additionally, did approximately $350 \mathrm{~km}$ preparing the ski-way at $\mathrm{km} 270$. It ran without a fault.

\section{Alpine ski-doo AL5 \\ Hour meter Start 185 \\ Odometer Start 4680 \\ Fuel Consumption: $3 \mathrm{Km} / \mathrm{Liter}$ \\ Finish 390 \\ Finish 5978 \\ Total 205 Hours \\ Total $1298 \mathrm{Km}$}

\section{Maintenance}

This machine was used by the survey (advance) party. They experienced ice in the fuel after 45 days, probably, as a result of an accumulation of moisture from blowing snow during fuel transfer. The earth wire between engine and chassis over heated few times, but the over heating did not cripple our progress.

\section{Alpine ski-doo AL7 \\ Hour meter Start 0 \\ Odometer Start 1515 \\ Finish 210 \\ Finish 1641 \\ Total 210 Hours \\ Total $210 \mathrm{Km}$ \\ Fuel Consumption: $3 \mathrm{Km} /$ Litre (presumed $1330 \mathrm{Km}$ from hourmeter).}

\section{Maintenance}

This machine was also used by the survey party. The speedo drive for the drive lug became loose in the transmission shaft stopping the speedo operation. Otherwise, 
the machine operated well. The fuel pump was mounted on the air box cover after being removed from the battery box. This new location meant that it was also very easy to see if the pump was operating correctly. (AL5 was also modified in this way).

\section{Generator PG $155 \mathrm{~kW}$}

Fuel Consumption: 2.3 Litres/Hour

Engine ran very well and the alternator never gave trouble even when blowing snow made generating difficult. The alternator started eating the wiring after 2.5 weeks, and was sent back to Scott Base. It was an ideal unit for this experiment because it had plenty of capacity and never had to work to its maximum.

\section{Generator PG 52.5 kW}

This unit was used very little. The alternator failed and was sent back on one of the Twin Otter flights.

\section{Generator FG $83.0 \mathrm{~kW}$}

Fuel Consumption: 1.4 Litres/Hour

This generator ran poorly at this altitude. The engine operated intermittently, because of a jamming exhaust valve. This fault was possibly due to the high operating temperature of the engine, caused by the wrong ignition timing. Information from Scott Base and Christchurch indicated, that it needed to be modified for altitude (injection timing advancement).

\section{Generator FG $73.0 \mathrm{~kW}$}

Fuel Consumption: 1.4 Litres/Hour

This machine was running hot and erratically similar to FG 8, also due to its ignition timing being standard. Its operation under load was smooth, the engine was very reliable but the alternator failed in blowing snow conditions.

\section{Generator FG $12.5 \mathrm{~kW}$}

Fuel Consumption: 0.8 Litres/hour

This machine came up to us with modified fuel ignition timing and ran very well without problems. The only cause of power outage was blowing snow conditions. The major differences between this unit and FG 7 and 8 was its improved economy and its smaller power output . It was generally fully loaded with just one $1.5 \mathrm{~kW}$ heater going, and could not charge batteries at the same time. After lowering the power consumption of one of the fan heaters to $0.5 \mathrm{~kW}$, it could charge simultaneously some batteries.

\section{Maudheims}

Both Maudheims worked well and gave no trouble. It would have been more convenient to have a draw bar available, because the plow was pulled behind one of the sledges.

\section{Box Sledge}

We had one sleepy sledge, which carried the detonators, and did not experience problems with it on the traverse.

\section{Anares}

These sleds had various problems none of which stopped our operation. As they were subsequently returned to New Zealand, I believe the problems would have been noted.

\section{VEHICLE USAGE TOTALS}

Vehicles 690 Hours




\title{
Summary and recommendations
}

\author{
Maudheims \\ Possibly consider the fitting of a drawbar on at least one Maudheim, as it then could be \\ selected if a drawbar is required for an event. \\ Vehicle Preparation \\ Preparation time depends on the size and type of the experiment. The two week lead \\ time before put in for this experiment should be considered the minimum.
}

\section{Ski-doos}

Although we had a good run with the ski-doos, the failure we experienced with AL3 would have been a big problem if it had happened earlier or if it had occurred in one of the machines used by the survey party. A ski foot part should be included in the spares in future deep field experiments, to be fitted in the field should failure occur. Alternatively, the ski leg should be stripped down prior to going into the field, to check for fatigue.

\section{Generators}

Yanmar

We had two different problems with FG 7 and 8. Both machines would benefit from being modified if working at this altitude in the future, however, with their reduction in output from $3 \mathrm{~kW}$ to $1.5 \mathrm{~kW}-1.7 \mathrm{~kW}$ their output is probably too small to be suitable for events of this type.

\section{$P G 15$ \& Onan's}

This type of generator worked well, with ease of starting and smaller output capacity losses being in their favor.

Our overall generator loading problems were probably two fold. First, we underestimated the required load of the science gear. Second, we underestimated the total losses due to the altitude with the operation of the Yanmar Diesel.

The hassle and unreliability of generating in blowing snow conditions can only be overcome by housing the generating equipment. As this is difficult with the smaller portable generators, a single higher capacity unit $6 \mathrm{~kW}-10 \mathrm{~kW}$ may be more suitable, which is housed in a shack. If essential loading is not high enough for overnight operation this could be increased with vehicle heating to maintain a suitable load for the generator.

\section{Acknowledgments}

Thanks must go to many people in McMurdo for their assistance with this event. Billy Stranger was happy to come and assist with the transmission problems we had with 069 Tucker. Thanks, also to Dale Willett from the heavy shop for his continuous support during the event, and to Ron Nugent and Peter Hokenstad from MEC for their help and advice in setting up equipment. I must also mention Jan de Vries, for his support, help and inventiveness with all things mechanical at every stage of the event. At Scott Base the work of Gus McAlistair, Jeremy Ridgen, Grant West, and all base staff was greatly appreciated. 